LA W REN CE LIVERM ORE N A TIO NAL LABORATORY

Finite Deformation of Magnetoelastic Film

M. I. Barham

May 31, 2011 
This document was prepared as an account of work sponsored by an agency of the United States government. Neither the United States government nor Lawrence Livermore National Security, LLC, nor any of their employees makes any warranty, expressed or implied, or assumes any legal liability or responsibility for the accuracy, completeness, or usefulness of any information, apparatus, product, or process disclosed, or represents that its use would not infringe privately owned rights. Reference herein to any specific commercial product, process, or service by trade name, trademark, manufacturer, or otherwise does not necessarily constitute or imply its endorsement, recommendation, or favoring by the United States government or Lawrence Livermore National Security, LLC. The views and opinions of authors expressed herein do not necessarily state or reflect those of the United States government or Lawrence Livermore National Security, LLC, and shall not be used for advertising or product endorsement purposes.

This work performed under the auspices of the U.S. Department of Energy by Lawrence Livermore National Laboratory under Contract DE-AC52-07NA27344. 


\title{
Finite Deformation of Magnetoelastic Film
}

\author{
by \\ Matthew Ian Barham \\ A dissertation submitted in partial satisfaction of the \\ requirements for the degree of \\ Doctor of Philosophy \\ in \\ Engineering - Mechanical Engeineering \\ in the \\ Graduate Division \\ of the \\ University of California, Berkeley \\ Committee in charge: \\ Professor David Steigmann, Chair \\ Professor David Bogy \\ Professor Tarek Zohdi \\ Professor John Wilkening
}

Spring 2011 
Finite Deformation of Magnetoelastic Film

Copyright 2011

by

Matthew Ian Barham 


\begin{abstract}
Finite Deformation of Magnetoelastic Film

by

Matthew Ian Barham

Doctor of Philosophy in Engineering - Mechanical Engeineering

University of California, Berkeley

Professor David Steigmann, Chair
\end{abstract}

A nonlinear two-dimensional theory is developed for thin magnetoelastic films capable of large deformations. This is derived directly from three-dimensional theory. Significant simplifications emerge in the descent from three dimensions to two, permitting the self field generated by the body to be computed a posteriori. The model is specialized to isotropic elastomers with two material models. First weak magnetization is investigated leading to a free energy where magnetization and deformation are un-coupled. The second closely couples the magnetization and deformation. Numerical solutions are obtained to equilibrium boundary-value problems in which the membrane is subjected to lateral pressure and an applied magnetic field. An instability is inferred and investigated for the weak magnetization material model. 


\section{To Erin Barham}

For supporting me throughout the entire process. 


\section{Contents}

List of Figures $\quad$ iv

List of Tables viii

1 Introduction $\quad 1$

2 3D Magnetoelasticity 3

2.1 Basic Equations . . . . . . . . . . . . . . . . . . . 3

2.2 Legendre Transformation . . . . . . . . . . . . . . . . . . . . . . . . . . . . . . . . . . . . . . . . . . .

2.3 Energy Identity and Weak Forms . . . . . . . . . . . . . . . . . . . . 12

2.4 Strong Form . . . . . . . . . . . . . . . . . . . . . . 13

2.5 Stability and Strong Ellipticity . . . . . . . . . . . . . . . . 15

2.6 Reduced Constitutive Equations and Isotropic Materials . . . . . . . . . . 17

3 Membrane Approximation $\quad 19$

3.1 Membrane Approximation . . . . . . . . . . . . . . . . . . . . . . 19

3.2 The Leading-Order Model . . . . . . . . . . . . . . . . . . . . . . . . . . . . . . . . . . . . . . .

3.3 Estimate of the Self Field . . . . . . . . . . . . . . . . . . . . . . . . . . . . . . . . . . . . . .

3.4 Loading . . . . . . . . . . . . . . . . . . . . . . . . . 25

3.5 Solvability of the Constraints . . . . . . . . . . . . . . . . 26

3.6 Lyapunov Functions . . . . . . . . . . . . . . . . . . . . . . . 29

4 Finite Difference Method $\quad 32$

5 Weakly Magnetized Material $\quad 40$

5.1 Shooting Method . . . . . . . . . . . . . . . . . . 42

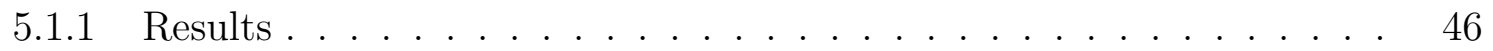

5.2 Variational Method . . . . . . . . . . . . . . . . 50

5.3 Finite Element Method . . . . . . . . . . . . . . . . . . 56

5.4 Finite Difference Method . . . . . . . . . . . . . . . . . 65

5.4 Results............................ 66 
6 Magnetoelastic Strain-Energy Function $\quad 72$

6.1 Results . . . . . . . . . . . . . . . . . . . . 73

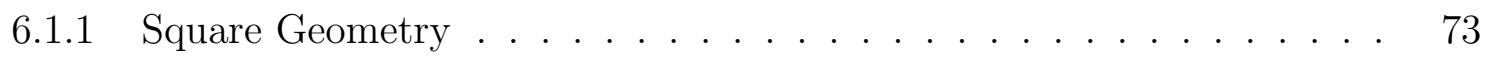

6.1.2 Circular Geometry . . . . . . . . . . . . . . . . . . . 90

7 Conclusion $\quad 102$

$\begin{array}{ll}\text { Bibliography } & 104\end{array}$ 


\section{List of Figures}

2.1 General Deformation of a body. . . . . . . . . . . . . . . . 4

3.1 Reference Membrane. . . . . . . . . . . . . . . . . . . . . 20

4.1 Unit cell of finite difference grid node with four neighboring nodes. . . . . . 33

4.2 Butter fly grid, where the "center" grid + "edge" grid = full grid. . . . . . . 34

4.3 Close up of finite difference grid node with three neighboring nodes. . . . . 34

5.1 Geometry for shooting method . . . . . . . . . . . . . . . . . . . 44

5.2 Shooting method equilibrium axisymmetric deformation for $D=0.00269 \mathrm{Am}^{2}$, for a range of dipole heights $\left(z_{d}\right) \ldots \ldots \ldots \ldots$. . . . . . . . . . 47

5.3 Shooting method equilibrium z-displacment at the center of the film for $D=$

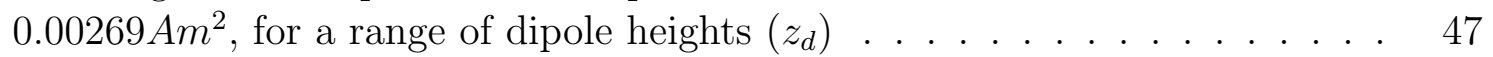

5.4 Shooting method equilibrium axisymmetric deformation for $z_{d}=0.5 \mathrm{~cm}$, for a range of dipole strengths $(\mathrm{D})$. . . . . . . . . . . . . . . . . . . . 48

5.5 Shooting method equilibrium $z$-displacement at the center of the film, for $z_{d}=0.5 \mathrm{~cm}$, for range of dipole strengths (D) . . . . . . . . . . . . 49

5.6 Degrees of freedom used in the variational method. . . . . . . . . . . . . 52

5.7 Energy (joules) versus the pole deformation $\zeta_{0}$ for various dipole heights above the reference plane. . . . . . . . . . . . . . . . . 54

5.8 Energy (joules) versus $\zeta_{0}$ versus $b$ for $\zeta_{d}=2.33$, and $D=0.02688 A m^{2}$. Stable equilibrium marked with a dot, $b^{\text {min }}=0.37931$ and $\zeta_{0}^{\text {min }}=0.41966 . \quad \ldots . \quad 55$

5.9 Energy (joules) versus $\zeta_{0}$ versus $b$ for $\zeta_{d}=2.2$. No equilibria present. . . . . 55

5.10 Energy (joules) versus $\zeta_{0}$ versus $b$ for $\zeta_{d}=\zeta_{i}=2.2427$. Incipient unstable equilibrium marked with a dot, $b^{\text {min }}=0.65517$ and $\zeta_{0}^{\text {min }}=0.62448 . . \quad \ldots . \quad 56$

5.11 Top view of FEM material regions (air is white, steel is black, and film is gray). 58

5.12 Side view of FEM material regions (air is white, steel is black, and film is gray) and the region allowed to advect is inside the dashed line. . . . . . . . 59

5.13 Deformed film and magnetic field (orange vectors) for the FEM model at $D_{e q}=0.00544 A m^{2} \ldots \ldots \ldots \ldots \ldots$. . . . . . . . . . . . . . . . . . . . . 61

$5.142 \mathrm{D}$ cross section of displacement for the FEM model. . . . . . . . . . . . . 62 
5.15 Comparison of displacement, shooting method vs. FEM. The black curves are the shooting method results and the blue curves are the FEM results for

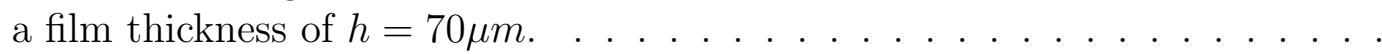

5.16 Comparison of the $z$-displacement at the center of the film for the two methods as a function of dipole strength. This particular FEM result used a film thickness of $h=70 \mu \mathrm{m}$.

5.17 Comparison of the FEM results, with different film thicknesses, to the shooting method. . . . . . . . . . . . . . . . . 64

5.18 "Butterfly" meshes considered . . . . . . . . . . . . . . . . . . . . 67

5.19 Mesh Refinement study at $D=0.00466 A^{2} \ldots \ldots$. . . . . . . . . . . 68

5.20 Maximum nodal artificial velocity vs artificial time for $D=0.00466 \mathrm{Am}^{2}$. . . 68

5.21 Film center height displacement vs. artificial time for $D=0.00466 \mathrm{Am}^{2}$. . . 69

$5.223 \mathrm{D}$ deformation of film using finite difference for $N_{c}=64$ and $D=0.00466 \mathrm{Am}^{2}$. 70

5.23 Cross-sectional deformation for a variety of dipole strengths for the finite difference method

5.24 Comparison of the $z$-displacement at the center of the film for the membrane model solved with the shooting method (Shoot) and finite difference (FD).

6.1 Square membrane center displacement for a variety of meshes defined by $N$ for $D=160 \times 10^{-6} A m^{2}$ and zero pressure. . . . . . . . . . . . . . .

6.2 Maximum nodal $z$-displacement for $D=160 \times 10^{-6} A m^{2}$ for a square membrane. . . . . . . . . . . . . . . . . . . 74

6.3 Maximum nodal velocity for $D=160 \times 10^{-6} A m^{2}$ for a square membrane. $\quad$. $\quad 75$

6.4 Deformed square membrane for $D=160 \times 10^{-6} A m^{2}$. . . . . . . . . . . 76

6.5 Reference magnetization (M) for $D=160 \times 10^{-6} A m^{2}$ on the reference square membrane. . . . . . . . . . . . . . . . . . . . 77

6.6 In-plane part, e, of director field, at $D=160 \times 10^{-6} A m^{2}$ for a square membrane. . . . . . . . . . . . . . . . . . . . 77

6.7 Current magnetization $(\mathbf{m})$ for $D=160 \times 10^{-6} A m^{2}$ on the deformed square

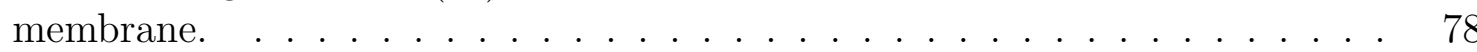

6.8 Self field in space at $D=160 \times 10^{-6} A m^{2}$, in the plane defined by $u_{2}=0$ for a square membrane. . . . . . . . . . . . . . . . . . .

6.9 Self field in space at $D=160 \times 10^{-6} A \mathrm{~m}^{2}$, in the plane defined by $z=3.0 \mathrm{~mm}$ for a square membrane. . . . . . . . . . . . . . . . . . .

6.10 Equilibrium displacement of a square membrane for a cross section $\left(\right.$ at $\left.u_{2}=0\right)$ for a variety of dipole strengths, where the values of $D$ given in the figure are multiplied by $\times 10^{-6} \mathrm{Am} \mathrm{m}^{2}$.

6.11 Equilibrium displacement of a square membrane for a cross section (at $u_{1}=$ $u_{2}$ ) for a variety of dipole strengths, where the values of $D$ given in the figure are multiplied by $\times 10^{-6} A m^{2}$. 
6.12 Equilibrium displacement of a square membrane for a cross section (at $u_{2}=0$ ) for a variety of dipole strengths with a pre-stretch of 1.2 , where the values of $D$ given in the figure are multiplied by $\times 10^{-6} \mathrm{Am}^{2}$.

6.13 Equilibrium displacement of a square membrane for a cross section (at $u_{1}=$ $u_{2}$ ) for a variety of dipole strengths with a pre-stretch of 1.2 , where the values of $D$ given in the figure are multiplied by $\times 10^{-6} A m^{2}$. . . . . . . . . . .

6.14 Equilibrium displacement of a square membrane for a cross section (at $u_{2}=0$ ) for a variety of dipole strengths subjected to a constant pressure, where the values of $D$ given in the figure are multiplied by $\times 10^{-6} \mathrm{Am}^{2}$. . . . . . . . .

6.15 Equilibrium displacement of a square membrane for a cross section (at $u_{1}=$ $u_{2}$ ) for a variety of dipole strengths subjected to a constant pressure, where the values of $D$ given in the figure are multiplied by $\times 10^{-6} \mathrm{Am}^{2}$. . . . . . .

6.16 Equilibrium displacement of a square membrane for a cross section (at $u_{2}=0$ ) for a variety of dipole strengths with a pressure dependent on the volume, where the values of $D$ given in the figure are multiplied by $\times 10^{-6} \mathrm{Am}^{2}$. . . .

6.17 Equilibrium displacement of a square membrane for a cross section (at $u_{1}=$ $u_{2}$ ) for a variety of dipole strengths with a pressure dependent on the volume, where the values of $D$ given in the figure are multiplied by $\times 10^{-6} \mathrm{Am}^{2}$. . . .

6.18 Membrane displacement under the dipole source, as a function of dipole strength. Effect of pre-stretch indicated by circles $(0)$ and crosses $(x)$; effect of fixed or volume dependent pressure is indicated by dots $(\cdot)$ and stars $(*)$, respectively (see text). . . . . . . . . . . . . . . .

6.19 Maximum nodal $z$-displacement for $D=170 \times 10^{-6} A m^{2}$ versus time for square membrane.

6.20 Maximum nodal velocity for $D=170 \times 10^{-6} A m^{2}$ versus time for square membrane.

6.21 Deformed square membrane for $D=240 \times 10^{-6} A m^{2}$ and dipole position of $\mathbf{y}_{d}=\{4.0,0.0,8.0\} \mathrm{mm} . \ldots \ldots \ldots \ldots$

6.22 Deformed square membrane for $D=240 \times 10^{-6} A m^{2}$ and dipole position of $\mathbf{y}_{d}=\{4.0,0.0,8.0\} \mathrm{mm} \ldots \ldots \ldots \ldots \ldots$

6.23 Equilibrium displacement of a circular membrane for a cross section (at $u_{2}=$ 0 ) for a variety of dipole strengths with a dipole position of $\mathbf{y}_{d}=\{4.0,0.0,8.0\} \mathrm{mm}$, where the values of $D$ given in the figure are multiplied by $\times 10^{-6} \mathrm{Am}^{2}$. . . . 89

6.24 Circular membrane center displacement for a variety of meshes for $D=180 \times$ $10^{-6} A m^{2}$ and zero pressure. . . . . . . . . . . . . . . . . . . . 90

6.25 Deformed circular membrane for $D=200 \times 10^{-6} A m^{2}$. . . . . . . . . . . 91

6.26 Reference magnetization $(\mathbf{M})$ for $D=200 \times 10^{-6} A m^{2}$ on the reference circular membrane. . . . . . . . . . . . . . . . . .

6.27 Unknown portion of the director (e) for $D=160 \times 10^{-6} A m^{2}$ on the reference circular membrane. . . . . . . . . . . . . . . . . . 
6.28 Current magnetization $(\mathbf{m})$ for $D=200 \times 10^{-6} A m^{2}$ on the deformed circular

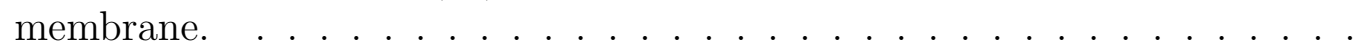

6.29 Equilibrium displacement of a circular membrane for a cross section (at $u_{2}=$ 0 ) for a variety of dipole strengths, where the values of $D$ given in the figure are multiplied by $\times 10^{-6} A m^{2}$. . . . . . . . . . . . . . . . .

6.30 Equilibrium displacement of a circular membrane for a cross section (at $u_{2}=$ 0 ) for a variety of dipole strengths with pre-stretch of 1.1, where the values of $D$ given in the figure are multiplied by $\times 10^{-6} A m^{2} . \ldots . . . . . .$.

6.31 Equilibrium displacement of a circular membrane for a cross section (at $u_{2}=$ 0 ) for a variety of dipole strengths subjected to a constant pressure, where the values of $D$ given in the figure are multiplied by $\times 10^{-6} \mathrm{Am}^{2} . \quad \ldots$. . .

6.32 Equilibrium displacement of a circular membrane for a cross section (at $u_{2}=$ 0 ) for a variety of dipole strengths with a pressure dependent on the volume, where the values of $D$ given in the figure are multiplied by $\times 10^{-6} \mathrm{Am}^{2}$. . . . 98

6.33 Comparison of a circular membrane maximum $z$-displacement. . . . . . . . 98

6.34 Deformed circular membrane for $D=300 \times 10^{-6} \mathrm{Am}^{2}$ and dipole position of $\mathbf{y}_{d}=\{4.0,0.0,8.0\} \mathrm{mm} \ldots \ldots \ldots \ldots$

6.35 Deformed circular membrane for $D=300 \times 10^{-6} A m^{2}$ and dipole position of $\mathbf{y}_{d}=\{4.0,0.0,8.0\} \mathrm{mm} \ldots \ldots \ldots \ldots \ldots$

6.36 Equilibrium displacement of a circular membrane for a cross section (at $y=0)$ for a variety of dipole strengths with a dipole position of $\mathbf{y}_{d}=\{4.0,0.0,8.0\} \mathrm{mm}$, where the values of $D$ given in the figure are multiplied by $\times 10^{-6} \mathrm{Am}^{2}$. . . 


\section{List of Tables}

$5.1 \zeta_{i}$ values and per cent error for the one- and two-parameter representations. . 53 


\section{Acknowledgments}

I would like to thank my advisor David Steigmann and mentor Dan White for their guidance throughout my PhD process. Also, Robert Rudd and Mike McElfresh for the inspiration for this project from their interest in magnetoelastic films. Lawrence Livermore National Laboratory for their support as part of the Computational Chemistry \& Materials Science Summer Institute and The Lawrence Scholar Program. My lab mates Ryan Krone for support and collaboration with coursework and research. My lovely wife for moving to the Bay Area and never ending support and motivation to complete my PhD. My parents Mark and Mary Ann Barham for their emotional support. My brother Jesse Barham for support and editing. As well as all of my friends and family for there support.

I would also like to thank the following publishers for publishing my dissertation research: Springer for publishing "Finite deformation of a pressurized magnetoelastic membrane in a stationary dipole field" in Acta Mechanica; IOP Science for publishing "Limit-point instability of a magnetoelastic membrane in a stationary magnetic field" in Smart Materials and Structures; IEEE for publishing "Finite-Element Modeling of the Deformation of a Thin Magnetoelastic Film Compared to a Membrane Model" in IEEE Transactions on Magnetics;

and Elsevier for publishing "Finite-element modeling of the deformation of magnetoelastic film" in the Journal of Computational Physics.

This work performed under the auspices of the U.S. Department of Energy by Lawrence Livermore National Laboratory under Contract DE-AC52-07NA27344. 


\section{Chapter 1}

\section{Introduction}

There is considerable current interest among mechanicians in nonlinear magnetoelasticity [7], [8], [9], [10], and [20] . This is due to the development of highly deformable magnetizable materials synthesized from elastomers infused with micro- or nano-scopic ferrous particles [19]. Such materials are capable of large deformations induced by magnetic fields. This property may be used to facilitate controlled pumping of fluid, for example, via remote actuation.

In the present work we develop an approximate membrane theory for magnetizable materials of this kind and apply it to simulate the interplay between an applied magnetic field modeled here as a remote dipole source and the pressure transmitted to the membrane by a confined gas. Attention is confined here to equilibria.

Two material models will be examined. First a simple model of weak magnetization will be derived, where the magnetization and mechanical stress are uncoupled. The second material model examined was proposed by Kankanala \& Triantafyllidis in [20] and [21], where here the magnetization and mechanical stress are closely coupled.

Chapter 2 contains a summary of three-dimensional magnetoelasticity and its specialization to isotropic elastomers. A corresponding membrane model is derived in Chapter 3 directly from the equations of the three-dimensional theory. This approach is more systematic than alternative formulations based, for example, on thickness-wise integration of the local three-dimensional balance laws (e.g. [12]). It incorporates a constraint requiring the magnetization to remain tangential to the film as it deforms. This is motivated by the fact such states are energetically optimal in thin films ([11] and [34]). Likewise, we impose the constraint of bulk incompressibility, and thus exclude dilatational modes of deformation that are energetically unfavorable in typical elastomers. However, unlike incompressibility, the constraint on magnetization is not of the kind that requires a reactive Lagrange multiplier in the relevant constitutive equation. Rather, it is a restriction involving the deformation, allowing local membrane geometry to adjust in response to an applied field. Constraints on the deformation of the Kirchhoff-Love type are typically imposed at the outset in theories of thin magnetoelastic plates [41] . However, in general such constraints impede the attainment 
of minima of the overall energy because, by confining attention to states of magnetization that are optimal at any deformation, we effectively eliminate magnetization as an independent variable. The bias induced by an applied field then yields deformations that violate constraints of the Kirchhoff-Love type. Here, this is addressed via a director field which emerges naturally from the underlying three-dimensional theory in the manner described in [36] for the purely mechanical problem, without restricting the nature of the deformation in thin bodies.

In Chapter 4 we define a finite-difference method to discretize the model spatially and discuss the solution of the resulting equations by the method of dynamic relaxation, in which equilibria are obtained as long-time limits of solutions to an artificial dynamical system with viscosity. This finite-difference method is then expanded to better handle circular films, by the introduction of a "butterfly" mesh.

In Chapter 5 we look at the the weak material model and solve for the equilibrium deformation using a shooting method (where we further simplify the problem to axisymmetric deformations), a finite element method and the finite-difference method discussed in Chapter 4. An instability is inferred from the deformation response and then further investigated using a variational method.

The finite-difference method described in Chapter 4 is applied, in Chapter 6 , to determine the deformation, magnetization and magnetic field generated by a thin film, using the Kankanala \& Triantafyllidis material model, in response to an applied magnetic field, pre-stretch and pressure load. 


\section{Chapter 2}

\section{D Magnetoelasticity}

This chapter consists of a summary of three-dimensional magnetoelasticity. Some of the work in this chapter appears in a paper by Steigmann [34]. The summary is intended to make the connection between the present work and the "accepted" formulation in Kovetz $[23]$.

\subsection{Basic Equations}

We start with a non-polarized magnetic elastomeric body, this body occupies region $\kappa$ in the reference configuration that is in a three-dimensional Euclidean space $\mathcal{E}$ identified with an inertial laboratory frame $\Sigma$. The reference configuration has an exterior unit normal $(\mathbf{N})$ to the surface of the body $\partial \kappa$. The bodies reference position is $\mathbf{x}$. The current position is determined from the reference position by:

$$
\mathbf{y}=\chi(\mathbf{x})
$$

where $\chi$ is the deformation function. The body occupies region $R$ in the current configuration with an exterior unit normal $(\mathbf{n})$ to surface of the body $(\partial R)$, see Figure 2.1. In the absence of an electric field the stress $\mathbf{T}$ in the body at rest in the current configuration $(R)$ is $[23]$

$$
\mathbf{T}=\hat{\mathbf{T}}+\mathbf{T}_{b}+(\mathbf{m} \cdot \mathbf{b}) \mathbf{I}-\mathbf{m} \otimes \mathbf{b}
$$

where

$$
\mathbf{T}_{b}=\mu_{0}^{-1}\left[\mathbf{b} \otimes \mathbf{b}-\frac{1}{2}(\mathbf{b} \cdot \mathbf{b}) \mathbf{I}\right]
$$

and

$$
\begin{gathered}
\hat{\mathbf{T}}=\rho\left(\phi_{\mathbf{F}}\right) \mathbf{F}^{t} \\
\mathbf{m}=-\rho \phi_{\mathbf{b}}
\end{gathered}
$$




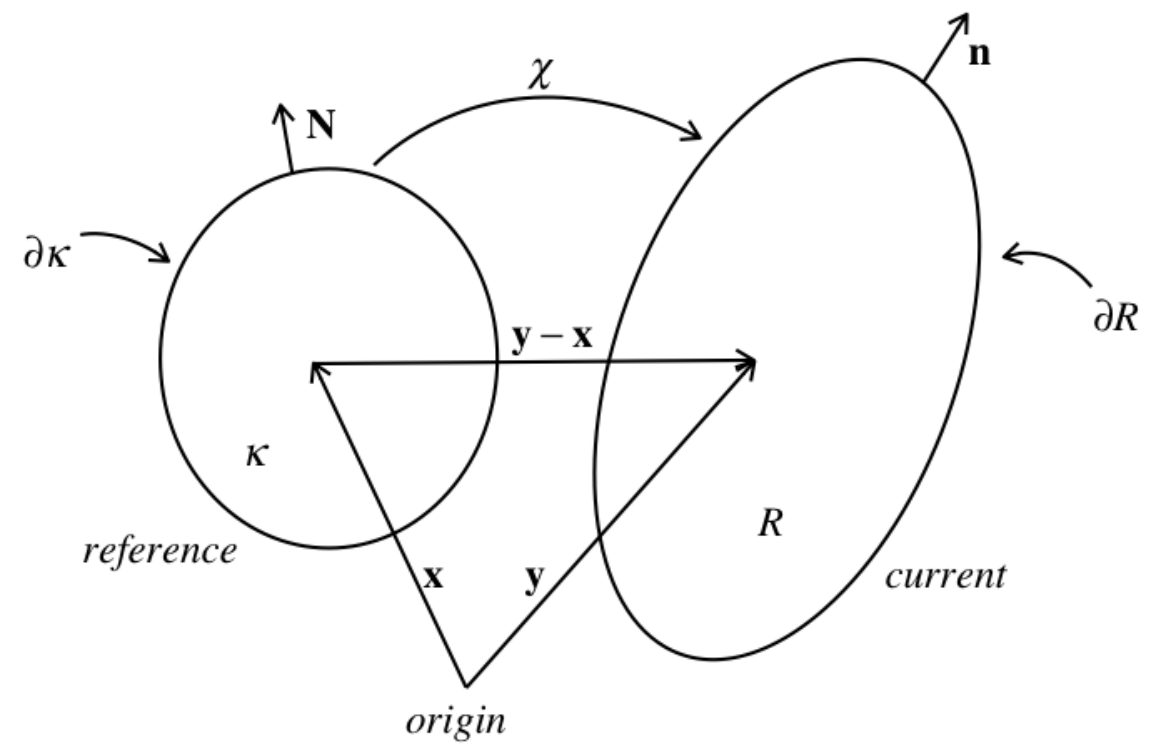

Figure 2.1: General Deformation of a body.

where $\mu_{0}(>0)$ is the permeability of free space, $\rho$ is the mass density in the current configuration, $\phi(\mathbf{F}, \mathbf{b})$ is the free energy per unit mass, $\mathbf{m}$ is the material magnetization per unit current volume, $\mathbf{b}$ is the magnetic induction, $\mathbf{F}=\operatorname{Grad}(\chi(\mathbf{x}))$ is the deformation gradient, Grad is the gradient with respect to the reference position (e.g. $\left.\operatorname{Grad}(\mathbf{a})=\frac{\partial\left(a_{i}\right)}{\partial x_{j}} \mathbf{e}_{i} \otimes \mathbf{e}_{j}\right)$ and I is the three dimensional identity on the translation space $E^{\prime}$ of $\mathcal{E}$.

Here and henceforth, bold subscripts identify derivatives with respect to a tensor or vector (e.g. $\left.\phi_{\mathbf{b}}=\partial \phi / \partial \mathbf{b}\right)$. The superscript ${ }^{t}$ is used to denote the transpose; the superscript ${ }^{-t}$ to denote the inverted transpose. All vectors are elements of $E^{\prime}$, and all tensors are mappings from $E^{\prime}$ back to $E^{\prime}$. A dot $(\cdot)$ between variables refers to the standard Euclidean inner product and the notation $\mathbf{a} \otimes \mathbf{b}$ is used to denote the tensor product of vectors defined by the rule $(\mathbf{a} \otimes \mathbf{b}) \mathbf{v}=(\mathbf{b} \cdot \mathbf{v}) \mathbf{a}$.

Maxwell's equations in the absence of currents are [23]

$$
\begin{aligned}
& \operatorname{div} \mathbf{b}=0 \quad \text { in } \quad \mathcal{E}, \\
& \operatorname{curl} \overline{\mathbf{h}}=\mathbf{0} \quad \text { in } \quad \mathcal{E} \backslash \bar{R}
\end{aligned}
$$

where $\overline{\mathbf{h}}$ is the spatial magnetic field vector, applying to electromagnetic fields in the absence of currents, div is the divergence with respect to the current position (e.g. diva $\left.=\frac{\partial\left(a_{i}\right)}{\partial y_{i}}\right)$, curl is the curl operator with respect to the current position (e.g. $\left.\operatorname{curl}(\mathbf{a})=\frac{\partial}{\partial \mathbf{y}} \times \mathbf{a}\right)$ and $\bar{R}$ is the closure of $R$. The aether relation of relevance is [23]

$$
\overline{\mathbf{h}}=\mu_{0}^{-1} \mathbf{b}
$$


In view of $2.3,2.6,2.8$ and the identity

$$
\operatorname{div}\left(\mathbf{b} \otimes \mathbf{b}-\frac{1}{2}(\mathbf{b} \cdot \mathbf{b}) \mathbf{I}\right)=\mathbf{b}(\operatorname{div} \mathbf{b})-\mathbf{b} \times \operatorname{curl} \mathbf{b}
$$

it is seen that

$$
\operatorname{div}\left(\mathbf{T}_{\mathbf{b}}\right)=\mathbf{0} \quad \text { in } \quad \mathcal{E} \backslash \bar{R}
$$

This only holds outside of the body. Inside the body there are "bound" currents associated with magnetization.

In the absence of free currents the relevant Maxwell equation is

$$
\operatorname{curlh}=\mathbf{0} \quad \text { in } \quad R
$$

where the associated free charge current potential (the magnetic field) is

$$
\mathbf{h}=\mu_{0}^{-1} \mathbf{b}-\mathbf{m} .
$$

From 2.11 it follows that there exists $\varphi(\mathbf{y}) \in R$ where

$$
\mathbf{h}=-\operatorname{grad} \varphi \quad \text { in } \quad R
$$

where grad is the gradient with respect to the current position (e.g. $\left.\operatorname{grad}(a)=\frac{\partial(a)}{\partial y_{i}} \mathbf{e}_{i}\right)$ Since there is no material magnetization outside the body 2.8 and 2.12 are equivalent outside the body

$$
\overline{\mathbf{h}}(\mathbf{y})=\mathbf{h}(\mathbf{y}) \quad \text { in } \quad \mathcal{E} \backslash \bar{R}
$$

With this relation the conclusion is made that the magnetic field $\mathbf{h}(\mathbf{y})$ can be extended to the space outside the body. The relation 2.13 can also be extended outside the body with a continuous extension of $\varphi$, giving

$$
\overline{\mathbf{h}}=-\operatorname{grad} \varphi \quad \mathcal{E} \backslash \bar{R}
$$

From this relation it can be seen that 2.7 is identically satisfied. There must be an allowance for a discontinuity in $\operatorname{grad} \varphi$ to exist on $\partial R$ since neither 2.7 nor 2.11 apply on the boundary.

The balance laws in the material that are relevant to solving a problem are 2.6 and 2.11 in addition to [23]:

$$
\operatorname{div} \mathbf{T}=\rho \ddot{\mathbf{y}} \quad \text { in } \quad R
$$

which is the local form of Euler's first postulate of equilibrium in the absence of distributed body force. If we look at a sub-volume $P$ with boundary $\partial P$ equation 2.16 with the use of the divergence theorem becomes

$$
\int_{\partial P} \mathbf{t} d a=\int_{P} \rho \ddot{\mathbf{y}} d v=\mathbf{0}
$$


where

$$
\mathbf{t}=\mathbf{T n}
$$

$\mathbf{t}$ is the traction and $\mathbf{n}$ is the external unit normal to $\partial P$. The relation between stress and traction is due to Noll's theorem [26] and the classical Cauchy theorem applied to 2.16. The meaning of equilibrium here and in general for electrodynamics is taken to indicate that an arbitrary region $P$ is stationary with respect to $\Sigma$ and that there is no dependence on time in the electromagnetic field.

The divergence theorem is used to express 2.6 in the global form while Stokes's theorem is used to express 2.11 in the global form

$$
\int_{\partial P} \mathbf{b} \cdot \mathbf{n} d a=0
$$

and

$$
\oint_{C} \mathbf{h} \cdot d \mathbf{y}=0
$$

where $C$ is an arbitrary simple closed circuit. If a field is discontinuous on the surface $S$ within the arbitrary region $P$ the jump conditions are obtained by shrinking $P$ onto $S$ leading to the jump conditions

$$
[\mathbf{b}] \cdot \mathbf{n}=0
$$

and

$$
\mathbf{n} \times[\mathbf{h}]=0
$$

where $[\cdot]=(\cdot)^{+}-(\cdot)^{-}$is the discontinuity at the surface of discontinuity $S$ in the direction n. Superscripts + and - refer to the limits of $(\cdot)$ at $\mathbf{y} \in S$ approached from $P^{+}$and $P^{-}$ respectively. Also $\mathbf{n}$ is the normal to $S$ in the direction from $P^{-}$to $P^{+}$.

If there is a discontinuity in $P$ the local form of Euler's first postulate 2.16 will not hold and the jump conditions at the discontinuity surface $S$ are obtained from the global form 2.17, leading to

$$
[\mathbf{T}] \mathbf{n}=\mathbf{0}
$$

Boundary conditions for the body $\bar{R}$ are obtained by admitting a discontinuity at the boundary with normal $\mathbf{n}$. Applying 2.21 and 2.22 to the boundary $\partial R$ leads to

$$
\mathbf{b}_{o} \cdot \mathbf{n}=\mathbf{b}_{i} \cdot \mathbf{n}
$$

and

$$
\mathbf{n} \times \mathbf{h}_{o}=\mathbf{n} \times \mathbf{h}_{i}
$$

where subscript ${ }_{o}$ and ${ }_{i}$ refer to boundary values of the field outside and inside the body respectively. From 2.12 we arrive at

$$
[\mathbf{h}]=(\mathbf{n} \cdot[\mathbf{h}]) \mathbf{n}=(\mathbf{n} \cdot \mathbf{m}) \mathbf{n},
$$


with $\mathbf{m}$ being evaluated on the boundary $\partial R$. From 2.6, 2.12, 2.13 and 2.15 we arrive at

$$
\begin{aligned}
\text { divh } & =-d i v \mathbf{m} \text { in } R \\
& =0 \quad \text { in } \mathcal{E} \backslash \bar{R} .
\end{aligned}
$$

The magnetic field is the sum

$$
\mathbf{h}=\mathbf{h}_{a}+\mathbf{h}_{s}
$$

of an applied field $\mathbf{h}_{a}$, generated by remote sources, and the self field $\mathbf{h}_{s}$ generated by the magnetized body. In the present circumstances both satisfy the relevant Maxwell equation without time derivatives; thus,

$$
\operatorname{curl} \mathbf{h}_{a}=\mathbf{0}
$$

in all of three-space and

$$
\operatorname{curl} \mathbf{h}_{s}=\mathbf{0}
$$

in $\mathcal{E} \backslash \partial R$. By the definition of the applied magnetic field we can regard it as the field that would exist if the body were not present and that the body has no influence on this part of the magnetic field. Thus the jump in the applied field at the boundary $\partial R$ is

$$
\left[\mathbf{h}_{a}\right]=\mathbf{0} \quad \text { on } \quad \partial R,
$$

and the applied magnetic field satisfies Maxwells equation 2.6

$$
\operatorname{div} \mathbf{h}_{a}=\mathbf{0} \text { in } \mathcal{E},
$$

Thus with the use of equation 2.26 the self field and the magnetization are subject to the jump condition

$$
\left[\mathbf{h}_{s}\right]=(\mathbf{n} \cdot \mathbf{m}) \mathbf{n} \quad \text { on } \quad \partial R,
$$

and to Maxwell's equation 2.27

$$
\begin{aligned}
\operatorname{div} \mathbf{h}_{s} & =-d i v \mathbf{m} \text { in } R \\
& =0 \text { in } \mathcal{E} \backslash \bar{R} .
\end{aligned}
$$

The field $\mathbf{h}_{a}$ is assumed to be assigned as a function that is continuously differentiable everywhere in $\mathcal{E}$ except at a finite number of singularities in $\mathcal{E} \backslash \bar{R}$.

In Chapters 5 and 6 the applied magnetic field will be generated by a dipole source

$$
\mathbf{h}_{a}(\mathbf{y})=D \frac{3(\mathbf{a} \cdot \mathbf{k}) \mathbf{a}-\mathbf{k}}{\ell^{3}},
$$

where the (signed) constant $D$ is the dipole strength having units of amps $\times$ meters $^{2}\left(A m^{2}\right)$, $\ell$ is the distance from the source to the point with position $\mathbf{y}$ in three-space, and $\mathbf{a}$ is the radial unit vector from the source to the considered point. Thus,

$$
\ell \mathbf{a}=\mathbf{y}-\mathbf{y}_{d},
$$


in which $|\mathbf{a}|=1$, is position measured from the source, located at $\mathbf{y}_{d}$ and $|\cdot|$ is the induced norm. This has an isolated singularity at the source. To compute the gradient of the applied field for use later we proceed from the fact that $d \mathbf{h}_{a}=\left(\operatorname{grad} \mathbf{h}_{a}\right) d \mathbf{y}$, where $d \mathbf{y}=(d \ell) \mathbf{a}+\ell d \mathbf{a}$. Using $\mathbf{a} \cdot d \mathbf{a}=0$ we obtain

$$
d \ell=\mathbf{a} \cdot d \mathbf{y} \quad \text { and } \quad \ell d \mathbf{a}=\Pi d \mathbf{y}
$$

where

$$
\Pi=\mathbf{I}-\mathbf{a} \otimes \mathbf{a}
$$

is the projection onto the plane orthogonal to a at position $\mathbf{y}$. These are used in the expression

$$
d \mathbf{h}_{a}=3 D\left\{\ell^{-3}[(\mathbf{a} \cdot \mathbf{k}) d \mathbf{a}+(\mathbf{k} \cdot d \mathbf{a}) \mathbf{a}]-\left[3 \ell^{-1}(\mathbf{a} \cdot \mathbf{k}) \mathbf{a}-\mathbf{k}\right] d \ell\right\}
$$

to deduce that

$$
\operatorname{grad} \mathbf{h}_{a}=3 D \ell^{-4}\{[(\mathbf{a} \cdot \mathbf{k}) \mathbf{I}+\mathbf{a} \otimes \mathbf{k}] \Pi-[3(\mathbf{a} \cdot \mathbf{k}) \mathbf{a}-\mathbf{k}] \otimes \mathbf{a}\} .
$$

This result is confirmed by verifying that $\operatorname{grad} \mathbf{h}_{a}=\left(\operatorname{grad} \mathbf{h}_{a}\right)^{t}$, which is equivalent to Maxwell's equation 2.6.

From equation 2.11 a potential $\varphi$ is defined where

$$
\mathbf{h}=-\operatorname{grad} \varphi
$$

This potential can also be broken into two parts,

$$
\varphi=\varphi_{a}+\varphi_{s}
$$

The first $\varphi_{s}$, which is the potential of the magnetic field produced by the body

$$
\mathbf{h}_{s}=-\operatorname{grad} \varphi_{s}
$$

From equations 2.33 and 2.34

$$
\left[\operatorname{grad} \varphi_{s}\right]=-(\mathbf{n} \cdot \mathbf{m}) \mathbf{n} \quad \text { on } \quad \partial R
$$

and

$$
\begin{aligned}
\operatorname{div}\left(\operatorname{grad}\left(\varphi_{s}\right)\right. & =-\operatorname{div} \mathbf{m} \text { in } R \\
& =0 \text { in } \mathcal{E} \backslash \bar{R} .
\end{aligned}
$$

The unique solution of $\varphi_{s}$ satisfying $\varphi_{s} \simeq|\mathbf{y}|^{-1}$ as $|\mathbf{y}| \rightarrow \infty$ is [18]

$$
4 \pi \varphi_{s}(\mathbf{y})=\int_{\partial R} \frac{\mathbf{m}(\overline{\mathbf{y}}) \cdot \mathbf{n}(\overline{\mathbf{y}})}{|\mathbf{y}-\overline{\mathbf{y}}|} d a(\overline{\mathbf{y}})-\int_{R} \frac{\operatorname{div} \mathbf{m}(\overline{\mathbf{y}})}{|\mathbf{y}-\overline{\mathbf{y}}|} d v(\overline{\mathbf{y}}) \quad \text { for } \quad \mathbf{y} \notin \partial R .
$$

The second $\varphi_{a}$ is the potential of the applied magnetic field $\mathbf{h}_{a}=\operatorname{grad} \varphi_{a}$. 
From the separation of magnetic field (equation 2.28) we conclude that

$$
\mathbf{h}_{a}=\mu_{0}^{-1} \mathbf{b}_{a},
$$

with the total magnetic induction

$$
\mathbf{b}=\mathbf{b}_{a}+\mathbf{b}_{s}
$$

where $\mathbf{b}_{s}$ is the magnetic induction induced by the body that satisfies 2.12

$$
\mu_{0}^{-1} \mathbf{b}_{s}=\mathbf{m}+\mathbf{h}_{s} \quad \text { in } \quad R
$$

and

$$
\mu_{0}^{-1} \mathbf{b}_{s}=\mathbf{h}_{s} \quad \text { in } \quad \mathcal{E} \backslash \bar{R}
$$

The jump condition on $\partial R$ gives

$$
\left(\mathbf{b}_{s}\right)_{o} \cdot \mathbf{n}=\left(\mathbf{b}_{s}\right)_{i} \cdot \mathbf{n} \quad \text { on } \quad \partial R,
$$

with $\mathbf{b}_{s}$ decaying as $\mathbf{y}$ gets large in the form $\left|\mathbf{b}_{s}\right| \simeq|\mathbf{y}|^{-2}$ as $|\mathbf{y}| \rightarrow \infty$

The jump condition 2.22 can be expressed as

$$
\mathbf{t}_{a}+\left(\mathbf{T}_{b}\right)_{o} \mathbf{n}=\mathbf{T}_{i} \mathbf{n}
$$

where $\mathbf{t}_{a}$ is the traction applied to the body on the boundary $\partial R,\left(\mathbf{T}_{b}\right)_{o}$ is the stress caused by the magnetic field outside the body and $\mathbf{T}_{i}$ is the total material stress on the boundary as approached from the inside of the body. We assume that $\mathbf{t}_{a}$ can be applied independently of the field external to the body. $\mathbf{t}_{a}$ may also be regarded as the reaction stress applied on the outside of $\partial R$ if the position is prescribed on the boundary.

Euler's second postulate of equilibrium is [23]:

$$
\int_{\partial R} \mathbf{y} \times \mathbf{t} d a=\int_{R} \mathbf{y} \times \rho \ddot{\mathbf{y}} d v,
$$

and this implies symmetry of $\mathbf{T}$ when ever 2.17 is satisfied

$$
\mathbf{T}^{t}=\mathbf{T},
$$

this imposes a constraint on the free energy.

\subsection{Legendre Transformation}

The free energy per unit mass $\phi(\mathbf{F}, \mathbf{b})$ as a function of $\mathbf{F}$ and $\mathbf{b}$ can be expressed as a function of other variables. It is also possible to have a free energy per unit mass in terms of $\mathbf{m}, \mathbf{h}$, or $\mu$ instead of $\mathbf{b}$, where $\mu$ is the material magnetization per unit mass defined by 
$\mathbf{m}=\rho \mu$. We will show the relation between the different free energy functions and their derivatives. From equation 2.12, the relation between the constitutive equations can be established as

$$
\mathbf{b}=\hat{\mathbf{b}}(\mathbf{F}, \mathbf{h})=\mu_{0}(\mathbf{h}+\hat{\mathbf{m}}(\mathbf{F}, \mathbf{h}))
$$

The first Legendre transformation is [23]

$$
\rho \psi(\mathbf{F}, \mathbf{h})=\rho \phi(\mathbf{F}, \mathbf{b})+\frac{1}{2} \mu_{0} \mathbf{m} \cdot \mathbf{m},
$$

with $\mathbf{m}$ and $\mathbf{b}$ give by constitutive equations in terms of $\mathbf{F}$ and $\mathbf{h}$. Consider a parameterized path $\Gamma=\mathbf{F}(u), \mathbf{h}(u)$. Take derivatives of 2.56 with respect to $u$ giving

$$
\rho \dot{\psi}=\dot{\rho}(\phi-\psi)+\rho \dot{\phi}+\mu_{0} \mathbf{m} \cdot \dot{\mathbf{m}},
$$

where $(\cdot) \cdot=\partial(\cdot) / \partial u$. Using the identity $\dot{\rho}=-\rho \mathbf{F}^{-t} \cdot \dot{\mathbf{F}}$ with the inner product of $2^{\text {nd }}$ order tensors $\mathbf{A} \cdot \mathbf{B}=\operatorname{trace}\left(\mathbf{A B}^{t}\right), 2.5$ and 2.57 become

$$
\left(\rho \phi_{\mathbf{F}}-\rho \psi_{\mathbf{F}}+\frac{1}{2} \mu_{0}(\mathbf{m} \cdot \mathbf{m}) \mathbf{F}^{-t}\right) \dot{\mathbf{F}}-\left(\rho \psi_{\mathbf{h}}+\mu_{0} \mathbf{m}\right) \dot{\mathbf{h}}=0 .
$$

Since $\dot{\mathbf{F}}$ and $\dot{\mathbf{h}}$ are independent of each other in the above equation the first and second term must equal zero independently yielding

$$
\rho \phi_{\mathbf{F}}=\rho \psi_{\mathbf{F}}+\frac{1}{2} \mu_{0}(\mathbf{m} \cdot \mathbf{m}) \mathbf{F}^{-t}
$$

and

$$
\rho \psi_{\mathbf{h}}=-\mu_{0} \mathbf{m}
$$

The stress 2.2 must be cast in terms of $\psi$ using equations $2.4,2.12$ and 2.60

$$
\mathbf{T}=\rho\left(\psi_{\mathbf{F}}\right) \mathbf{F}^{t}+\mathbf{T}_{h}+\mu_{0} \mathbf{h} \otimes \mathbf{m}
$$

where

$$
\mathbf{T}_{h}=\mu_{0}\left[\mathbf{h} \otimes \mathbf{h}-\frac{1}{2}(\mathbf{h} \cdot \mathbf{h}) \mathbf{I}\right]
$$

From 2.3, 2.12 and 2.62 it is seen that

$$
\mathbf{T}_{h}=\mathbf{T}_{b} \quad \text { in } \quad \mathcal{E} \backslash \bar{R}
$$

Using 2.11 it is seen that

$$
\operatorname{div} \mathbf{T}_{h}=\mu_{0} \mathbf{h}(\operatorname{div} \mathbf{h}) .
$$

We will define a new stress that will be used later in the derivation of the weak form of the equilibrium equation, namely

$$
\mathbf{T}_{m}=\mathbf{T}_{h}+\mu_{0} \mathbf{h} \otimes \mathbf{m}
$$


with 2.63 leading to

$$
\mathbf{T}_{m}=\mathbf{T}_{b} \quad \text { in } \quad \mathcal{E} \backslash \bar{R} .
$$

Using equation 2.12 it is seen that

$$
\operatorname{div} \mathbf{T}_{m}=\mu_{0}(\operatorname{grad} \mathbf{h}) \mathbf{m} .
$$

The second Legendre transformation is [23]

$$
\hat{\xi}(\mathbf{F}, \mu)=\psi(\mathbf{F}, \mathbf{h})+\frac{1}{2} \mu_{0} \mu \cdot \mathbf{h},
$$

In the same manner that 2.58 was derived, a relation between the derivatives of $\hat{\xi}(\mathbf{F}, \mu)$ and $\psi(\mathbf{F}, \mathbf{h})$ is derived leading to

$$
\left(\hat{\xi}_{\mathbf{F}}-\psi_{\mathbf{F}}\right) \dot{\mathbf{F}}+\left(\hat{\xi}_{\mu}+\mu_{0} \mathbf{h}\right) \dot{\mu}=0 .
$$

In a similar manner the first and second terms equal zero independently leading to

$$
\hat{\xi}_{\mathbf{F}}=\psi_{\mathbf{F}}
$$

and

$$
\hat{\xi}_{\mu}=\mu_{0} \mathbf{h}
$$

When separating the magnetic field as in 2.28 and using 2.43 the magnetization and magnetic field are related constitutively by

$$
\hat{\xi}_{\mu}=\mu_{0} \mathbf{h}=\mu_{0}\left(\mathbf{h}_{a}-\operatorname{grad} \varphi_{s}\right) .
$$

With the following relation the free energy per unit mass $\xi(\mathbf{F}, \mathbf{m})$ as a function of $\mathbf{F}$ and m can easily be obtained.

$$
\hat{\xi}(\mathbf{F}, \mu)=\hat{\xi}\left(\mathbf{F}, \rho^{-1} \mathbf{m}\right)=\xi(\mathbf{F}, \mathbf{m})
$$

The derivatives are related by

$$
\hat{\xi}_{\mu}=\rho \xi_{\mathbf{m}}
$$

and

$$
\hat{\xi}_{\mathbf{F}}=\xi_{\mathbf{F}}
$$

If the idealization is made that the material is incompressible as is done for highly elastic materials 2.70 becomes

$$
\hat{\xi}_{\mathbf{F}}=\psi_{\mathbf{F}}+q \mathbf{F}^{-t}
$$

where $q$ is a constraint pressure associated with incompressibility to be determined when solving the system of equations with the addition of the equation restricting the density in the manner

$$
\rho(\chi(\mathbf{x}, t), t)=\rho_{\kappa}(\mathbf{x}) ; \quad \text { equivalently, } \quad J=1, \quad \text { where } \quad J=\operatorname{det} \mathbf{F} .
$$


where $\rho_{\kappa}$ is the mass density of the reference configuration. The constraint pressure if the material is incompressible also appears in the $\mathbf{F}$-derivative of $\phi$ and $\psi$ multiplied by $\mathbf{F}^{-t}$, and the term $\frac{1}{2} \mu_{0}(\mathbf{m} \cdot \mathbf{m})$ can be absorbed into $q$, leading to

$$
\phi_{\mathbf{F}}=\psi_{\mathbf{F}}+q \mathbf{F}^{-t}
$$

The constraint pressure term $q$ also appears in the stress which needs to be added to 2.2 and 2.61 multiplied by $\mathbf{I}$. The constraint pressures are subject to 2.16 and 2.52 .

The total stress $\mathbf{T}$ for incompressible material is

$$
\mathbf{T}=\rho\left(\xi_{\mathbf{F}}\right) \mathbf{F}^{t}+\mu_{0}\left(\mathbf{h} \otimes \mathbf{h}-\frac{1}{2}|\mathbf{h}|^{2} \mathbf{I}\right)+\mu_{0} \mathbf{h} \otimes \mathbf{m}-q \mathbf{I} .
$$

\subsection{Energy Identity and Weak Forms}

Before deriving the weak form of the balance equation we will look at the equation for the applied tractions on the boundary of the body 2.52 in light of derivations above. Using equations 2.61, 2.65 and 2.66, 2.52 can be expressed as

$$
\mathbf{t}_{a}=\rho\left(\psi_{\mathbf{F}}\right) \mathbf{F}^{t} \mathbf{n}-\left[\mathbf{T}_{m}\right] \mathbf{n} .
$$

Using 2.10 and 2.62 in 2.65 multiplied by the normal gives

$$
\mu_{0}^{-1}\left(\mathbf{T}_{m}\right) \mathbf{n}=\mu_{0}^{-1}(\mathbf{b} \cdot \mathbf{n}) \mathbf{h}-\frac{1}{2}(\mathbf{h} \cdot \mathbf{h}) \mathbf{n}
$$

Looking at the jump condition of the equation above and using 2.24 gives

$$
\mu_{0}^{-1}\left[\mathbf{T}_{m}\right] \mathbf{n}=\mu_{0}^{-1}\left(\mathbf{b}_{o} \cdot \mathbf{n}\right)[\mathbf{h}]-\frac{1}{2}\left(\mathbf{h}_{o} \cdot \mathbf{h}_{o}-\mathbf{h}_{i} \cdot \mathbf{h}_{i}\right) \mathbf{n}
$$

From 2.25 it is seen that

$$
\mathbf{h}_{o} \cdot \mathbf{h}_{o}-\mathbf{h}_{i} \cdot \mathbf{h}_{i}=\left(\mathbf{n} \cdot \mathbf{h}_{i}\right)^{2}-\left(\mathbf{n} \cdot \mathbf{h}_{o}\right)^{2},
$$

with the reduction based on 2.10 and 2.26 yielding

$$
\mathbf{h}_{o} \cdot \mathbf{h}_{o}-\mathbf{h}_{i} \cdot \mathbf{h}_{i}=(\mathbf{n} \cdot \mathbf{m})^{2}-2 \mu_{0}^{-1}\left(\mathbf{n} \cdot \mathbf{h}_{o}\right)(\mathbf{n} \cdot \mathbf{m}),
$$

Using the above and 2.26 in 2.82 we arrive at

$$
\left[\mathbf{T}_{m}\right] \mathbf{n}=\frac{1}{2} \mu_{0}^{-1}(\mathbf{m} \cdot \mathbf{n})^{2} \mathbf{n},
$$

and then 2.80 becomes

$$
\rho\left(\psi_{\mathbf{F}}\right) \mathbf{F}^{t} \mathbf{n}=\mathbf{t}_{a}+\frac{1}{2} \mu_{0}^{-1}(\mathbf{m} \cdot \mathbf{n})^{2} \mathbf{n} \quad \text { on } \quad \partial R .
$$


To obtain the weak form of the balance law in terms of the energy identity we form the inner product of 2.16 with $\dot{\mathbf{y}}$, where $\mathbf{y}=\chi(\mathbf{x}, u)$, for $\mathbf{x} \in \kappa$, is a parameterized deformation field with $u=0$ corresponding to an equilibrium state. Also, $(\cdot)=\partial(\cdot) / \partial u$ at a fixed $\mathbf{x}$, evaluated at $u=0$. With the use of 2.61 and 2.65 and integrating over the body yields

$$
\int_{R} \rho \psi_{\mathbf{F}} \cdot \dot{\mathbf{F}} d v=\int_{R} \operatorname{div}\left\{\left(\rho \psi_{\mathbf{F}} \mathbf{F}^{t}\right)^{t} \dot{\mathbf{y}}\right\} d v+\int_{R} \dot{\mathbf{y}} \cdot\left(\operatorname{div} \mathbf{T}_{m}-\rho \ddot{\mathbf{y}}\right) d v
$$

where

$$
\dot{\mathbf{F}}=\operatorname{Grad} \dot{\mathbf{y}}
$$

The above applies in the case of incompressibility provided

$$
\mathbf{F}^{-t} \cdot \operatorname{Grad} \dot{\mathbf{y}}=0
$$

with a constant pressure multiplied by $\mathbf{F}^{-t}$ subtracted from $\rho \psi_{\mathbf{F}}$ in the first integral and the use of the divergence theorem with 2.86 in the second integral of 2.87. Substitution of 2.67 into the third integral yields the weak form

$\int_{R}\left(\rho \psi_{\mathbf{F}}-q \mathbf{F}^{-t}\right) \cdot \dot{\mathbf{F}} d v=\int_{\partial R} \mathbf{t}_{a} \cdot \dot{\mathbf{y}} d a+\frac{1}{2} \mu_{0}^{-1} \int_{\partial R}(\mathbf{m} \cdot \mathbf{n})^{2} \dot{\mathbf{y}} d a+\mu_{0} \int_{R} \dot{\mathbf{y}} \cdot(\operatorname{gra} a d \mathbf{h}) \mathbf{m} d v-\int_{R} \rho \dot{\mathbf{y}} \cdot \ddot{\mathbf{y}} d v$.

The weak form based on the energy potential in the form $\xi(\mathbf{F}, \mathbf{m})$ is found by plugging equation 2.70 into 2.90

$$
\int_{R}\left(\rho \xi_{\mathbf{F}}-q \mathbf{F}^{-t}\right) \cdot \dot{\mathbf{F}} d v=\int_{\partial R} \mathbf{t}_{a} \cdot \dot{\mathbf{y}} d a+\frac{1}{2} \mu_{0}^{-1} \int_{\partial R}(\mathbf{m} \cdot \mathbf{n})^{2} \dot{\mathbf{y}} d a+\mu_{0} \int_{R} \dot{\mathbf{y}} \cdot(\operatorname{grad} \mathbf{h}) \mathbf{m} d v-\int_{R} \rho \dot{\mathbf{y}} \cdot \ddot{\mathbf{y}} d v .
$$

\subsection{Strong Form}

The strong or local form of the equation may be read off almost by inspection of equation 2.91. Thus

$$
\operatorname{div}\left[\rho\left(\xi_{\mathbf{F}}\right) \mathbf{F}^{t}-q \mathbf{I}\right]+\mu_{0}(\operatorname{grad} \mathbf{h}) \mathbf{m}=\rho \ddot{\mathbf{y}} \quad \text { in } \quad R .
$$

While compatible boundary data entail, for example, the satisfaction of

$$
\rho\left(\xi_{\mathbf{F}}\right) \mathbf{F}^{t} \mathbf{n}-q \mathbf{n}=\mathbf{t}_{a}+\frac{1}{2} \mu_{0}(\mathbf{m} \cdot \mathbf{n})^{2} \mathbf{n} \quad \text { on } \quad \partial R_{t}
$$

with $\mathbf{y}$ assigned on the complement $\partial R \backslash \partial R_{t}$.

Our further considerations require equations involving a referential divergence operator. For 2.92, this is easily achieved via the Piola transformation

$$
\mathbf{P}=\left[\rho\left(\xi_{\mathbf{F}}\right) \mathbf{F}^{t}-q \mathbf{I}\right] \mathbf{F}^{*}=W_{\mathbf{F}}-q \mathbf{F}^{*},
$$


where

$$
W(\mathbf{F}, \mathbf{m})=\rho_{\kappa} \xi
$$

is the referential strain-energy density, and

$$
\mathbf{F}^{*}=J \mathbf{F}^{-t}
$$

is the cofactor of the deformation gradient. Thus,

$$
\operatorname{Jdiv}\left[\rho\left(\xi_{\mathbf{F}}\right) \mathbf{F}^{t}-q \mathbf{I}\right]=\operatorname{Div} \mathbf{P}
$$

where Div is the referential divergence based on $\mathbf{x}$; therefore, 2.92 is equivalent to

$$
\operatorname{Div} \mathbf{P}+\mu_{0}(\operatorname{gradh}) \mathbf{m}=\rho \ddot{\mathbf{y}}
$$

in which $J=1$ has been imposed. Further, we find the referential form of the boundary condition 2.93 to be

$$
\mathbf{P N}=\mathbf{p}_{a}+\frac{1}{2} \mu_{0}(\mathbf{m} \cdot \mathbf{n})^{2} \mathbf{F}^{*} \mathbf{N} \quad \text { on } \partial \kappa_{t},
$$

where $\partial R_{t}=\chi\left(\partial \kappa_{t}\right)$, having used Nanson's formula

$$
\alpha \mathbf{n}=\mathbf{F}^{*} \mathbf{N}
$$

where $\alpha=\left|\mathbf{F}^{*} \mathbf{N}\right|$ is the local areal dilation of $\partial \kappa_{t}$. Here,

$$
\mathbf{p}_{a}=\alpha \mathbf{t}_{a}
$$

is the applied traction measured per unit area of $\partial \kappa_{t}$.

With the use of equations 2.72, 2.74 and 2.95 we see that the magnetization and magnetic field are related constitutively by [34]

$$
W_{\mathbf{m}}=\mu_{0} \mathbf{h}=\mu_{0}\left(\mathbf{h}_{a}-\operatorname{grad} \varphi_{s}\right)
$$

Thus, if the constitutive function $W(\mathbf{F}, \mathbf{m})$ is known, equations 2.43, 2.46, 2.98, 2.99 and 2.102 yield a coupled integro-differential system to be solved for the deformation and magnetization.

This presents considerable analytical and numerical challenges [11]. In Chapter 5 these are avoided by considering the limit of a weakly magnetized body in the presence of a strong applied field. In this limit the self field may be generated from 2.46 a posteriori, and plays only a passive role in the analysis. Alternatively, a direct simulation of the field may be based on a discretization of Maxwell's equations in the space surrounding the body [7] and $[5]$.

In Chapter 6 we use a result derived in [11] for thin films to show that the tractability of the formulation adopted in [2] is retained when the magnetization and applied fields are 
comparable in magnitude. This yields a conventional differential-algebraic system to be solved on a reference surface associated with the thin film.

To facilitate subsequent analysis, we use a pull-back $\mathbf{M}$ of $\mathbf{m}$ defined by

$$
\int_{s} \mathbf{m} \cdot \mathbf{n} d a=\int_{S} \mathbf{M} \cdot \mathbf{N} d A
$$

in which $S \subset \kappa$ is an arbitrary orientable surface and $s=\chi(S, t) \subset R$ is its image in the current configuration. Nanson's formula then furnishes

$$
\mathbf{M}=J \mathbf{F}^{-1} \mathbf{m} .
$$

In particular, this yields the convenient connections

$$
\alpha \mathbf{m} \cdot \mathbf{n}=\mathbf{M} \cdot \mathbf{N} \quad \text { and } \quad J \operatorname{div} \mathbf{m}=\operatorname{Div} \mathbf{M},
$$

which enable us to use, in place 2.99 and 2.46 respectively, the equivalent expressions

$$
\mathbf{P N}=\mathbf{p}_{a}+\frac{1}{2} \mu_{0} \alpha^{-2}(\mathbf{M} \cdot \mathbf{N})^{2} \mathbf{F}^{*} \mathbf{N} \quad \text { on } \quad \partial \kappa_{t},
$$

and

$$
4 \pi \varphi_{s}(\mathbf{y}, t)=\int_{\partial \kappa} \frac{\mathbf{M}(\mathbf{x}, t) \cdot \mathbf{N}(\mathbf{x})}{|\mathbf{y}-\chi(\mathbf{x}, t)|} d A(\mathbf{x})-\int_{\kappa} \frac{\operatorname{Div} \mathbf{M}(\mathbf{x}, t)}{|\mathbf{y}-\chi(\mathbf{x}, t)|} d V(\mathbf{x}), \quad \text { for } \quad \mathbf{x} \notin \partial \kappa,
$$

in which the role of time has been made explicit and incompressibility has been imposed.

\subsection{Stability and Strong Ellipticity}

A magneto-mechanical energy balance may be derived from 2.33, 2.34, 2.98, and 2.99 . Thus [34] and [18],

$$
\frac{d}{d t}\left\{K+\int_{R} \rho \xi d v+M-\mu_{0} \int_{R} \mathbf{h}_{a} \cdot \mathbf{m} d v\right\}=\int_{\partial R_{t}} \mathbf{t}_{a} \cdot \dot{\mathbf{y}} d a,
$$

where

$$
K=\frac{1}{2} \int_{R} \rho|\dot{\mathbf{y}}|^{2} d v
$$

is the conventional kinetic energy and [6], [7], and [34]

$$
M=-\frac{1}{2} \mu_{0} \int_{R} \mathbf{h}_{s} \cdot \mathbf{m} d v
$$


is the magnetostatic energy of the self field. In this work we consider conservative applied tractions for which

$$
\int_{\partial R_{t}} \mathbf{t}_{a} \cdot \dot{\mathbf{y}} d a=\frac{d}{d t} L,
$$

where $L$ is a suitable load potential. We then have the conservation law

$$
\frac{d}{d t} E^{\prime}=0, \quad \text { where } \quad E^{\prime}=K+E
$$

is the total magneto-mechanical energy in which

$$
E=\int_{R} \rho \xi d v+M-\mu_{0} \int_{R} \mathbf{h}_{a} \cdot \mathbf{m} d v-L
$$

is the magnetoelastic potential energy. We remark that our energy balance excludes certain terms that are present in the balance discussed in [18] These vanish collectively when the applied field is assigned as a stationary function of $\mathbf{y}$, as assumed here; that is, as a function which is independent of $t$ in the spatial description [34]. Further, the results of [34] may be used to show that the static specialization of 2.98, in which inertia is suppressed, furnishes an Euler-Lagrange equation for $E$.

In this work we consider pressure acting on a part $\partial R_{t}$ of the boundary formed by the union of two surfaces, $\partial R_{t}^{+}$and $\partial R_{t}^{-}$, having no points in common. Uniformly distributed pressures, $P^{+}$and $P^{-}$respectively, are acting on these surfaces. Let $S$ be a fixed orientable surface such that $\partial S=C$, the curve bounding $\partial R_{t}^{-}$. We choose $S$ such that its closure, and that of $\partial R_{t}^{-}$, intersect only in $C$, so that $S \cup \partial R_{t}^{-}$encloses a well-defined volume $V^{-} \subset \mathcal{E}$. In the applications of interest here, $\partial R_{t}^{+}$and $\partial R_{t}^{-}$respectively are the 'upper' and 'lower' lateral surfaces of a thin sheet which, together with $S$, contains a compressible gas that transmits a pressure $P^{-}$to the lower surface. In Chapter 5 and 6 we identify $S$ with the reference plane for the sheet. The upper surface is subjected to a fixed pressure $P^{+}$supplied by a large reservoir.

This loading is conservative, and the associated potential, modulo an unimportant constant, is [34]

$$
L=\int^{V^{-}} P^{-}(v) d v-P^{+}\left(V+V^{-}\right),
$$

where $P^{-}\left(V^{-}\right)$is the pressure-volume relation for the compressible gas and $V$ is the volume of the body in configuration $R$. In the present context, the incompressibility of the magnetoelastic material allows us to suppress $V$ on the right-hand side. Further,

$$
V^{-}=-\frac{1}{3} \int_{\partial \kappa_{t}^{-}} \mathbf{y} \cdot \mathbf{F}^{*} \mathbf{N} d A,
$$

where $\partial \kappa_{t}^{-}$is the pre-image of $\partial R_{t}^{-}$in the reference configuration with exterior unit normal $\mathbf{N}[34]$. 
In a full thermodynamic treatment accounting for dissipative effects, the energy balance 2.112 is replaced by the imbalance $d E^{\prime} / d t \leq 0$ [18], so that if a state with vanishing initial velocity tends asymptotically to an equilibrium state, then the latter minimizes the potential energy $E$ [18] and [20]; i.e., it furnishes a value of the potential energy not exceeding that supplied by the initial state. Because $K$ is a positive-definite function of the velocity, it follows that $E^{\prime}$ delivers a Lyapunov function for the dynamical system provided that the potential energy is strictly minimized at the equilibrium state. The considered equilibrium state is then stable. Without further qualification, this claim applies rigorously only to finitedimensional systems [22]. Thus, we apply it only to the system that has been discretized for the purpose of numerical analysis. This is the basis of a dynamic relaxation method for computing equilibria (Section 5.4 and Chapter 6).

In particular, then, an asymptotically stable equilibrium state minimizes the potential energy. In the purely mechanical setting, it is well known that a minimizing deformation necessarily satisfies the (local) strong-ellipticity inequality pointwise (see, for example, [28]). In the present setting this is replaced by the magnetoelastic strong-ellipticity inequalities $[20]$

$$
\mathbf{a} \cdot \mathbf{A}(\mathbf{b}) \mathbf{a}>0 \text { and } \mathbf{c} \cdot\left(W_{\mathbf{m m}}\right) \mathbf{c}>0,
$$

where $\mathbf{A}(\mathbf{b})$ is the acoustic tensor defined by

$$
\mathbf{a} \cdot \mathbf{A}(\mathbf{b}) \mathbf{a}=\mathbf{a} \otimes \mathbf{b} \cdot\left\{W_{\mathbf{F F}}-W_{\mathbf{F m}}\left(W_{\mathbf{m m}}\right)^{-1} W_{\mathbf{m F}}\right\}[\mathbf{a} \otimes \mathbf{b}] .
$$

These inequalities apply for all non-zero vectors $\mathbf{a}, \mathbf{b}, \mathbf{c}$, with $\mathbf{a}$ and $\mathbf{b}$ subject to the restriction

$$
\mathbf{a} \cdot \mathbf{F}^{*} \mathbf{b}=0
$$

associated with incompressibility. The second inequality implies that $W_{\mathbf{m m}}$ is invertible, as required by the first inequality. In terms of Cartesian components, inequalities $(2.116)_{1,2}$ are

$$
A_{i j}(\mathbf{b}) a_{i} a_{j}>0 \text { and }\left(\partial^{2} W / \partial m_{i} \partial m_{j}\right) c_{i} c_{j}>0,
$$

where

$$
A_{i j}(\mathbf{b})=\left\{\partial^{2} W / \partial F_{i A} \partial F_{j B}-\left(\partial^{2} W / \partial F_{i A} \partial m_{k}\right)\left(W_{\mathbf{m m}}\right)_{k l}^{-1}\left(\partial^{2} W / \partial m_{l} \partial F_{j B}\right)\right\} b_{A} b_{B} .
$$

They furnish pointwise restrictions on energy-minimizing states of deformation and magnetization jointly, which in turn play a central role in reducing the three-dimensional theory to a two-dimensional membrane model (Chapter 3).

\subsection{Reduced Constitutive Equations and Isotropic Ma- terials}

The restriction imposed on the stress $\mathbf{T}$ (equation 2.79) by equation 2.54 restricts the way in which $W$ can depend on its arguments. This requires:

$$
\left(W_{\mathbf{F}}\right) \mathbf{F}^{t}+W_{\mathbf{m}} \otimes \mathbf{m} \quad \text { is symmetric, }
$$


which is found, following [2], to be equivalent to the requirement:

$$
W(\mathbf{F}, \mathbf{m})=W(\mathbf{Q F}, \mathbf{Q m}) \text { for all rotations } \mathbf{Q},
$$

and this in turn is satisfied if and only if [7]

$$
W(\mathbf{F}, \mathbf{m})=\bar{W}(\mathbf{C}, \overline{\mathbf{M}})
$$

for some function $\bar{W}$, where

$$
\mathbf{C}=\mathbf{F}^{t} \mathbf{F} \quad \text { and } \quad \overline{\mathbf{M}}=\mathbf{F}^{t} \mathbf{m} .
$$

The latter is related to the pull-back $\mathbf{M}$ by (cf. 2.104)

$$
J \overline{\mathrm{M}}=\mathrm{CM},
$$

and so $W$ may be written as a (different) function of $\mathbf{C}$ and $\mathbf{M}$, if desired. We make use of this function in Chapter 3.

We assume the material to be isotropic, with a center of symmetry, relative to the reference configuration $\kappa$. Then [2],

$$
\bar{W}(\mathbf{C}, \overline{\mathbf{M}})=\bar{W}\left(\mathbf{R}^{t} \mathbf{C R}, \mathbf{R}^{t} \overline{\mathbf{M}}\right) \quad \text { for all orthogonal } \mathbf{R} .
$$

For $\mathbf{R}=-\mathbf{I}$ this yields $\bar{W}(\mathbf{C}, \overline{\mathbf{M}})=\bar{W}(\mathbf{C},-\overline{\mathbf{M}})$, which is satisfied if and only if $[9] \bar{W}(\mathbf{C}, \overline{\mathbf{M}})=$ $\hat{W}(\mathbf{C}, \overline{\mathbf{M}} \otimes \overline{\mathbf{M}})$ for some function $\hat{W}$ subject to the restriction

$$
\hat{W}(\mathbf{C}, \overline{\mathbf{M}} \otimes \overline{\mathbf{M}})=\hat{W}\left(\mathbf{R}^{t} \mathbf{C R}, \mathbf{R}^{t}(\overline{\mathbf{M}} \otimes \overline{\mathbf{M}}) \mathbf{R}\right) \quad \text { for all orthogonal } \mathbf{R} .
$$

For incompressible materials, standard representation theory [42] implies that $\hat{W}=U\left(I_{1}, I_{2}, I_{4}, I_{5}, I_{6}\right)$ for some function $U$, where

$$
I_{1}=t r \mathbf{C}, \quad I_{2}=\frac{1}{2}\left[I_{1}^{2}-t r\left(\mathbf{C}^{2}\right)\right], \quad I_{4}=\mathbf{C} \cdot \overline{\mathbf{M}} \otimes \overline{\mathbf{M}}, \quad I_{5}=\mathbf{C}^{2} \cdot \overline{\mathbf{M}} \otimes \overline{\mathbf{M}}, \quad I_{6}=\overline{\mathbf{M}} \cdot \overline{\mathbf{M}}
$$

Proceeding as in [34] we then obtain

$$
W_{\mathbf{F}}=2 \mathbf{F}\left(S y m \bar{W}_{\mathbf{C}}\right)+\mathbf{m} \otimes \bar{W}_{\overline{\mathbf{M}}} \quad \text { and } \quad W_{\mathbf{m}}=\mathbf{F} \bar{W}_{\overline{\mathbf{M}}},
$$

with

$$
\begin{aligned}
\operatorname{Sym} \bar{W}_{\mathbf{C}} & =\left(U_{1}-I_{1} U_{2}\right) \mathbf{I}+U_{2} \mathbf{C}+U_{4} \overline{\mathbf{M}} \otimes \overline{\mathbf{M}}+U_{5}[\mathbf{C}(\overline{\mathbf{M}} \otimes \overline{\mathbf{M}})+(\overline{\mathbf{M}} \otimes \overline{\mathbf{M}}) \mathbf{C}] \\
\text { and } \bar{W}_{\overline{\mathbf{M}}} & =2\left(U_{4} \mathbf{C}+U_{5} \mathbf{C}^{2}+U_{6} \mathbf{I}\right) \overline{\mathbf{M}},
\end{aligned}
$$

where $U_{k}=\partial U / \partial I_{k}$. 


\section{Chapter 3}

\section{Membrane Approximation}

\subsection{Membrane Approximation}

We consider a body whose reference configuration $\kappa$ is a prismatic region generated by the parallel translation of a simply-connected plane $\Omega$ with piecewise-smooth boundary curve $\partial \Omega$. The closure of $\kappa$ is $\bar{\Omega} \times[-h / 2, h / 2]$, where $\bar{\Omega}=\Omega \cup \partial \Omega$ and $h$ is the (uniform) thickness. Let $l$ be another length scale such as the diameter of a hole in $\Omega$ or a typical spanwise dimension. We assume that $\epsilon \doteq h / l \ll 1$, and, in the theoretical development, adopt $l$ as the unit of length $(l=1)$. We derive a two-dimensional membrane model by estimating the equations of the three-dimensional theory to leading order in $\epsilon$. Further, we suppose the deformation to be $C^{2}$ and the magnetization to be $C^{1}$ in the interior of the body, so that the local equations of the foregoing theory apply almost everywhere.

With minor loss of generality we assume the dipole in 2.35 to be orthogonal to the plane $\Omega$, which is thus oriented by the unit vector $\mathbf{k}$. The projection onto the plane is

$$
\mathbf{1}=\mathbf{I}-\mathbf{k} \otimes \mathbf{k}
$$

and generates the orthogonal decomposition

$$
\mathbf{P}=\mathbf{P} 1+\mathbf{P k} \otimes \mathbf{k}
$$

of the Piola transform 2.94. Let $\varsigma$ be a linear coordinate in the direction of $\mathbf{k}$, and suppose $\varsigma=0$ on $\Omega$. Equation 2.98 is then equivalent to

$$
\operatorname{Div}_{\|}(\mathbf{P} \mathbf{1})+\mathbf{P}^{\prime} \mathbf{k}+\mu_{0}(\operatorname{grad} \mathbf{h}) \mathbf{m}=\mathbf{0},
$$

where $(\cdot)^{\prime}=\partial(\cdot) / \partial \varsigma$ and $D i v_{\|}$is the (referential) two-dimensional divergence with respect to position $\mathbf{u}$ on $\Omega$, where

$$
\mathbf{x}=\mathbf{u}+\varsigma \mathbf{k}
$$

see Figure 3.1. This holds at all points in the interior of the body and therefore at $\varsigma=0$ in 


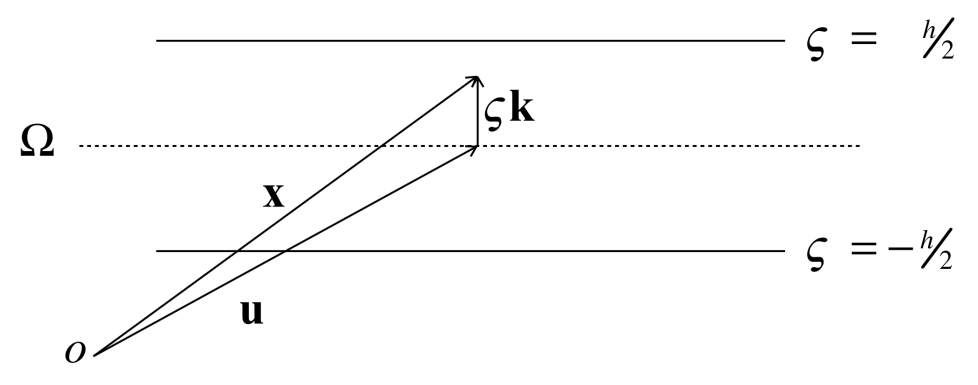

Figure 3.1: Reference Membrane.

particular. Thus,

$$
D i v_{\|}\left(\mathbf{P}_{0} \mathbf{1}\right)+\mathbf{P}_{0}^{\prime} \mathbf{k}+\mu_{0}(\operatorname{grad} \mathbf{h})_{0} \mathbf{m}_{0}=\mathbf{0},
$$

where the subscript ${ }_{0}$ identifies the values of functions at $\varsigma=0$; i.e., on the plane $\Omega$. For example,

$$
\mathbf{P}_{0}=W_{\mathbf{F}}\left(\mathbf{F}_{0}, \mathbf{m}_{0}\right)-q_{0} \mathbf{F}_{0}^{*} \text { and } \mu_{0} \mathbf{h}_{0}=W_{\mathbf{m}}\left(\mathbf{F}_{0}, \mathbf{m}_{0}\right)
$$

in which [36]

$$
\mathbf{F}_{0}=\mathbf{f}+\mathbf{d} \otimes \mathbf{k}
$$

where

$$
\mathbf{f}=\nabla \mathbf{r}
$$

$\mathbf{r}$ and $\mathbf{d}(\mathbf{u}, t)$ are the restrictions to $\Omega$ of $\chi$ and $\chi^{\prime}$, respectively, and $\nabla$ is the two-dimensional gradient on $\Omega$; i.e., the gradient with respect to $\mathbf{u}$. The current position of the deformed mid-surface is

$$
\mathbf{r}=\chi(\mathbf{u}, t)=\chi\left(\mathbf{x}_{0}, t\right)=\mathbf{y}_{0} .
$$

We note that $\mathbf{r}(\mathbf{u}, t)$ maps $\Omega$ to the deformed membrane surface $\omega=\chi(\Omega, t)$. Accordingly, $\mathbf{f}$ maps $\Omega^{\prime}$, the translation space associated with the plane $\Omega$, to $T_{\omega}$, the tangent plane to $\omega$ at the material point $\mathbf{u} \in \Omega$.

To accommodate the constraint of bulk incompressibility we impose

$$
1=\operatorname{det} \mathbf{F}_{0}=\mathbf{F}_{0}^{*} \mathbf{k} \cdot \mathbf{F}_{0} \mathbf{k}=\alpha \mathbf{n} \cdot \mathbf{d},
$$

where 3.7 and Nanson's formula 2.100 have been used in the final equality. Here, $\alpha$ is the local areal dilation of $\Omega$ and $\mathbf{n}$ is the orientation of the surface onto which $\Omega$ is deformed; i.e., the unit normal to $T_{\omega}$. The general solution is

$$
\mathbf{d}=\alpha^{-1} \mathbf{n}+\mathbf{f e},
$$

where $\mathbf{e} \in \Omega^{\prime}$ is arbitrary. Further, equations 2.100 and 3.7 yield

$$
\alpha \mathbf{n}=\mathbf{f i}_{1} \times \mathbf{f i}_{2},
$$


where $\mathbf{i}_{\alpha} \in \Omega^{\prime}$ are subject only to the requirement that $\left\{\mathbf{i}_{1}, \mathbf{i}_{2}, \mathbf{k}\right\}$ be a positively-oriented orthonormal set. Thus $\mathbf{F}_{0}$ is determined by $\mathbf{f}$ and $\mathbf{e}$, regarded as independent variables. The associated Cauchy-Green deformation tensor, $\mathbf{C}_{0}=\mathbf{F}_{0}^{t} \mathbf{F}_{0}$, is

$$
\mathbf{C}_{0}=\mathbf{c}+\mathbf{c e} \otimes \mathbf{k}+\mathbf{k} \otimes \mathbf{c e}+\left(\alpha^{-2}+\mathbf{e} \cdot \mathbf{c e}\right) \mathbf{k} \otimes \mathbf{k}, \quad \text { where } \quad \mathbf{c}=\mathbf{f}^{t} \mathbf{f}
$$

and $\alpha$ is obtained by evaluating the norm of 3.12, yielding

$$
\alpha=\sqrt{\operatorname{det} \mathbf{c}} .
$$

\subsection{The Leading-Order Model}

The foregoing equations, holding on $\Omega$, are exact consequences of the three-dimensional theory. Approximations arise in using them to represent material response in $\Omega \times[-\epsilon / 2, \epsilon / 2]$. Let $\mathbf{P}^{ \pm}$be the interior limits of $\mathbf{P}$ as $\varsigma \rightarrow \pm \epsilon / 2$, where the exterior unit normals are $\mathbf{N}^{ \pm}= \pm \mathbf{k}$. Their Taylor expansions yield

$$
\mathbf{P}^{+} \mathbf{N}^{+}+\mathbf{P}^{-} \mathbf{N}^{-}=\epsilon \mathbf{P}_{0}^{\prime} \mathbf{k}+o(\epsilon) \text { and } \quad \mathbf{P}^{+} \mathbf{N}^{+}-\mathbf{P}^{-} \mathbf{N}^{-}=2 \mathbf{P}_{0} \mathbf{k}+o(\epsilon) .
$$

On the left-hand sides we use 2.99 together with the estimates

$$
\left(\mathbf{F}^{*} \mathbf{N}\right)^{ \pm}= \pm\left(\mathbf{F}^{*}\right)^{ \pm} \mathbf{k}= \pm \mathbf{F}_{0}^{*} \mathbf{k}+(\epsilon / 2)\left(\mathbf{F}^{*}\right)_{0}^{\prime} \mathbf{k}+o(\epsilon)
$$

and

$$
\alpha^{ \pm}=\alpha_{0} \pm(\epsilon / 2) \alpha_{0}^{\prime}+o(\epsilon)
$$

where

$$
\alpha_{0}^{\prime}=\alpha_{0}^{-1} \mathbf{F}_{0}^{*} \mathbf{k} \cdot\left(\mathbf{F}^{*}\right)_{0}^{\prime} \mathbf{k},
$$

which follows on differentiation of $\alpha=\left|\mathbf{F}^{*} \mathbf{k}\right|$. After some algebra we obtain

$$
\mathbf{P}^{+} \mathbf{N}^{+}+\mathbf{P}^{-} \mathbf{N}^{-}=\mathbf{p}_{a}^{+}+\mathbf{p}_{a}^{-}+\epsilon \mu_{0} \alpha_{0}^{-2} M_{0}\left\{\left[M_{0}^{\prime}-\left(\alpha_{0}^{\prime} / \alpha_{0}\right) M_{0}\right] \mathbf{F}_{0}^{*} \mathbf{k}+\frac{1}{2} M_{0}\left(\mathbf{F}^{*}\right)_{0}^{\prime} \mathbf{k}\right\}+o(\epsilon)
$$

and

$$
\mathbf{P}^{+} \mathbf{N}^{+}-\mathbf{P}^{-} \mathbf{N}^{-}=\mathbf{p}_{a}^{+}-\mathbf{p}_{a}^{-}+\mu_{0} \alpha_{0}^{-2} M_{0}^{2} \mathbf{F}_{0}^{*} \mathbf{k}+O(\epsilon),
$$

where $\mathbf{p}_{a}^{ \pm}$are the applied tractions at the lateral surfaces and $M=\mathbf{M} \cdot \mathbf{k}$. The role of the latter suggests the decomposition

$$
\mathbf{M}=\mathbf{1} \mathbf{M}+M \mathbf{k},
$$

which yields

$$
\operatorname{Div} \mathbf{M}=\operatorname{Div}_{\|}(\mathbf{1} \mathbf{M})+M^{\prime} .
$$

It follows from 3.15 and 3.19 that 3.5 yields a well-defined differential equation in the limit of small $\epsilon$ only if $\mathbf{P}_{0}^{\prime} \mathbf{k}$ remains bounded. Further, 3.6 implies that the deformation 
gradient and magnetization are bounded on $\Omega$ only if $\mathbf{P}_{0}$ is bounded. From 3.19 and 3.20 it is therefore necessary that

$$
\mathbf{p}_{a}^{+}+\mathbf{p}_{a}^{-}=\epsilon \mathbf{p}+o(\epsilon) \quad \text { and } \quad \mathbf{p}_{a}^{+}-\mathbf{p}_{a}^{-}=2 \mathbf{q}+o(1)
$$

where $\mathbf{p}$ and $\mathbf{q}$ are of order unity in magnitude. It follows that, to leading order in $\epsilon$,

$$
\begin{aligned}
& \mathbf{P}_{0}^{\prime} \mathbf{k}=\mathbf{p}+\mu_{0} \alpha_{0}^{-2} M_{0}\left\{\left[M_{0}^{\prime}-\left(\alpha_{0}^{\prime} / \alpha_{0}\right) M_{0}\right] \mathbf{F}_{0}^{*} \mathbf{k}+\frac{1}{2} M_{0}\left(\mathbf{F}^{*}\right)_{0}^{\prime} \mathbf{k}\right\} \quad \text { and } \\
& \mathbf{P}_{0} \mathbf{k}=\mathbf{q}+\frac{1}{2} \mu_{0} \alpha_{0}^{-2} M_{0}^{2} \mathbf{F}_{0}^{*} \mathbf{k} .
\end{aligned}
$$

\subsection{Estimate of the Self Field}

Before proceeding we obtain an estimate of the leading-order self-field potential 2.107. An elementary calculation based on 3.21 and 3.22 gives

$$
\begin{aligned}
4 \pi \varphi_{s}(\mathbf{y}, t)= & \epsilon\left\{\int_{\partial \Omega} \frac{\mathbf{1 M}_{0} \cdot \nu}{|\mathbf{y}-\mathbf{r}|} d S-\int_{\Omega} \frac{\left[\operatorname{Div}_{\|}\left(\mathbf{1 M}_{0}\right)+M_{0}^{\prime}\right]}{|\mathbf{y}-\mathbf{r}|} d A\right\} \\
& +\int_{\partial \kappa^{+}} \frac{M^{+}}{\left|\mathbf{y}-\chi^{+}\right|} d A-\int_{\partial \kappa^{-}} \frac{M^{-}}{\left|\mathbf{y}-\chi^{-}\right|} d A+o(\epsilon)
\end{aligned}
$$

where the superscripts ${ }^{ \pm}$identify the values of functions at the upper and lower lateral surfaces $\partial \kappa^{ \pm}=\Omega \times\{ \pm \epsilon / 2\}$ and $\nu \in \Omega^{\prime}$ is the unit normal exterior to $\Omega$. This is valid provided that $\mathbf{y} \neq \mathbf{r}(\mathbf{u}, t)$ for any $\mathbf{u} \in \bar{\Omega}$. To estimate the associated integrals, we compute $|\mathbf{v}|^{\prime}=|\mathbf{v}|^{-1} \mathbf{v} \cdot \mathbf{v}^{\prime}$, where $\mathbf{v}=\mathbf{y}-\chi(\mathbf{x}, t)$ and the derivative is with respect to $\varsigma$ at fixed $\mathbf{y}$. Accordingly, $\mathbf{v}^{\prime}=-\mathbf{F k}$, and 3.7 gives

$$
|\mathbf{y}-\chi|_{0}^{\prime}=-\frac{(\mathbf{y}-\mathbf{r})}{|\mathbf{y}-\mathbf{r}|} \cdot \mathbf{d}
$$

For $\mathbf{y} \neq \mathbf{r}$ this yields

$$
\frac{1}{|\mathbf{y}-\chi|^{ \pm}}=\frac{1}{|\mathbf{y}-\mathbf{r}|}\left\{1 \pm \frac{\epsilon}{2} \frac{(\mathbf{y}-\mathbf{r})}{|\mathbf{y}-\mathbf{r}|^{2}} \cdot \mathbf{d}\right\}+o(\epsilon)
$$

which, when combined with

$$
M^{ \pm}=M_{0} \pm(\epsilon / 2) M_{0}^{\prime}+o(\epsilon)
$$

results in

$$
4 \pi \varphi_{s}(\mathbf{y}, t) / \epsilon=\int_{\partial \Omega} \frac{\mathbf{1 M}_{0} \cdot \nu}{|\mathbf{y}-\mathbf{r}|} d S+\int_{\Omega}\left[\frac{M_{0}}{|\mathbf{y}-\mathbf{r}|^{2}}(\mathbf{y}-\mathbf{r}) \cdot \mathbf{d}-\frac{D i v_{\|}\left(\mathbf{1 M}_{0}\right)}{|\mathbf{y}-\mathbf{r}|}\right] d A+o(\epsilon) / \epsilon
$$


provided that 3.27 is uniformly valid over the domain. This limitation effectively restricts the use of 3.29 to points $\mathbf{y}$ whose distances from the membrane are of order unity compared to $\epsilon$; that is, to points in space whose minimum distances from the deforming membrane surface are large compared to membrane thickness. Accordingly, it may not be used to describe the self field in the interior of the material.

To characterize the magnetic state inside the film, we estimate 3.22 at an interior point $\overline{\mathbf{x}} \in \kappa$. For points $\mathbf{x}$ near $\overline{\mathbf{x}}$, the presumed differentiability of the deformation implies that $|\overline{\mathbf{y}}-\chi(\mathbf{x}, t)|=O(|\xi|)$, where $\xi=\overline{\mathbf{x}}-\mathbf{x}$ and $\overline{\mathbf{y}}=\chi(\overline{\mathbf{x}}, t)$. The self field is obtained by computing the gradient of $\varphi_{s}$ with respect to $\mathbf{y}$ and evaluating the result at $\overline{\mathbf{y}}$; thus, for $\overline{\mathbf{x}} \notin \partial \kappa$,

$$
4 \pi \mathbf{h}_{s}(\overline{\mathbf{y}})=\int_{\partial \kappa} \frac{(\mathbf{M} \cdot \mathbf{N}) \mathbf{v}}{|\overline{\mathbf{y}}-\chi(\mathbf{x})|^{2}} d A-\int_{\kappa} \frac{(\operatorname{Div} \mathbf{M}) \mathbf{v}}{|\overline{\mathbf{y}}-\chi(\mathbf{x})|^{2}} d V, \quad \text { where } \quad \mathbf{v}=(\overline{\mathbf{y}}-\chi(\mathbf{x})) /|\overline{\mathbf{y}}-\chi(\mathbf{x})|,
$$

in which $t$ has been suppressed. The singularity is of order $|\xi|^{2}$, which is integrable in $\kappa$. Therefore the volume integral makes a contribution of order $\epsilon$. The boundary integral includes a contribution from the surface $\partial \Omega \times(-\epsilon / 2, \epsilon / 2)$, on which $|\overline{\mathbf{y}}-\chi(\mathbf{x})|$ is strictly bounded away from zero for any $\epsilon$. Accordingly, this too contributes at order $\epsilon$, leaving

$$
4 \pi \mathbf{h}_{s}(\overline{\mathbf{y}})=\int_{\partial \kappa^{+} \cup \partial \kappa^{-}} \frac{(\mathbf{M} \cdot \mathbf{N}) \mathbf{v}}{|\overline{\mathbf{y}}-\chi(\mathbf{x})|^{2}} d A+O(\epsilon)
$$

in which $\mathbf{M} \cdot \mathbf{N}= \pm M^{ \pm}$on $\partial \kappa^{ \pm}$, respectively. Thus,

$$
\left|\int_{\partial \kappa^{+} \cup \partial \kappa^{-}} \frac{(\mathbf{M} \cdot \mathbf{N}) \mathbf{v}}{|\overline{\mathbf{y}}-\chi(\mathbf{x})|^{2}} d A\right| \leq \int_{\partial \kappa^{+} \cup \partial \kappa^{-}} \frac{\left|M^{ \pm}\right|}{|\overline{\mathbf{y}}-\chi(\mathbf{x})|^{2}} d A \leq \max _{\partial \kappa^{+} \cup \partial \kappa^{-}}\left|M^{ \pm}\right| \int_{\partial \kappa^{+} \cup \partial \kappa^{-}} \frac{1}{|\overline{\mathbf{y}}-\chi(\mathbf{x})|^{2}} d A .
$$

The integrand in the final integral is dominated by its asymptotic behavior near $\overline{\mathbf{x}}$; i.e., by $|\xi|^{-2}$. For small thickness, the integral may then be shown to be $O(|\ln \epsilon|)$ in magnitude. In view of 3.28, the upper bound remains finite in the limit only if $\max _{\Omega}\left|M_{0}\right|=0$, in which case it is of order $|\epsilon \ln \epsilon|$. This guarantees that $\left|\mathbf{h}_{s}(\overline{\mathbf{y}})\right|$ is finite and vanishes with $\epsilon$. In particular, then,

$$
\mathbf{h}_{s} \text { vanishes on } \Omega \text {, at leading order. }
$$

The alternative $\left(M_{0} \neq 0\right)$ yields an upper bound of order $|\ln \epsilon|$, which allows the self field to grow without bound as thickness tends to zero. In this case the magnetostatic energy, and therefore the potential energy, may become unbounded. However, this alternative does not require the self field to become unbounded, and so our analysis, while suggestive, is not conclusive. In other words, we have only shown that the constraint:

$$
M_{0}=0 \quad \text { on } \Omega,
$$

is sufficient for 3.33 and for boundedness of the magnetostatic energy. Using 3.7 and 3.21, we then derive

$$
\mathbf{m}_{0} \in T_{\omega} \quad \text { on } \quad \omega, \quad \text { at every } \quad \mathbf{u} \in \Omega .
$$


To explore this issue further, consider the part of the potential energy involving magnetization. This is (cf. 2.110, 2.111 and 2.113)

$$
E_{\text {mag }}=\int_{\kappa}\left[W-\frac{1}{2}\left(\mathbf{h}+\mathbf{h}_{a}\right) \cdot \mathbf{m}\right] d V
$$

in which $\mathbf{y}$ and $\mathbf{F}$ are fixed, and reduces to

$$
E_{\text {mag }}=\int_{\kappa}\left(W-\mathbf{h}_{a} \cdot \mathbf{m}\right) d V
$$

if the self field is negligible; i.e., if 3.35 holds. Here the magnetization is obtained by solving 2.102 in which the self field is suppressed, so that $E_{m a g}$ is controlled entirely by the deformation. This effectively eliminates the magnetization as an independent variable. In the work of Gioia and James [11] on non-deforming films it is proved that minimizers of 3.36 furnish energies that converge to 3.37 as film thickness tends to zero. It was also proved that optimal states of magnetization necessarily satisfy 3.35 and that the residual self-field vanishes, in accordance with 3.33 (see also [34]). Further, in [11] it is shown that there is no residual magnetostatic equation to leading order; indeed, the solution 2.46 to 2.27 has already been used in the course of obtaining 3.35 and therefore plays no further role. These results imply that 3.33 and 3.35 characterize optimal states of magnetization in a sufficiently thin film, at any fixed deformation. In particular, the magnetostatic energy is negligible at leading order. The Euler equation for the deformation that emerges from this leading-order approximation is given by 2.98 [34], but with $\operatorname{gradh}$ replaced by $\operatorname{grad} \mathbf{h}_{a}$. This follows from the fact that the variational derivative of $\mathbf{h}_{a}$, identified by a superposed dot, is purely convective; i.e., $\dot{\mathbf{h}}_{a}=\left(\operatorname{grad} \mathbf{h}_{a}\right) \dot{\mathbf{y}}$, if the applied field is a stationary function of $\mathbf{y}$. The claim then follows from 2.29, which is equivalent to the symmetry of $\operatorname{grad} \mathbf{h}_{a}$. Strictly, these results are known to be necessary only for optimal (energy-minimizing) states of magnetization and may not apply to dynamical states. However, in this work we use dynamics solely to facilitate the computation of equilibria. We do not model actual dynamic interactions. Accordingly, we restrict attention to states of magnetization that are energetically optimal at any deformation, equilibrated or otherwise.

Equation 3.34 affords the important simplifications

$$
\mathbf{P}_{0}^{\prime} \mathbf{k}=\mathbf{p} \quad \text { and } \quad \mathbf{P}_{0} \mathbf{k}=\mathbf{q}
$$

For points remote from the deforming film 3.29 is applicable and simplifies, by virtue of 3.34 , to

$$
4 \pi \varphi_{s}(\mathbf{y}, t) / \epsilon=\int_{\Omega} \mathbf{1} \mathbf{M}_{0} \cdot \nabla\left(\frac{1}{|\mathbf{y}-\mathbf{r}|}\right) d A+o(\epsilon) / \epsilon,
$$

where $\nabla$ is the two-dimensional gradient with respect to $\mathbf{u} \in \Omega$ and Green's theorem has been used to combine terms. Proceeding as in the calculation leading to 3.29, we put $\mathbf{v}(\mathbf{u}, t)=\mathbf{y}-\mathbf{r}(\mathbf{u}, t)$ and use 3.8 to derive

$$
d\left(|\mathbf{v}|^{-1}\right)=-|\mathbf{v}|^{-3} \mathbf{v} \cdot d \mathbf{v}, \quad \text { where } \quad d \mathbf{v}=-d \mathbf{r}(\mathbf{u})=-\mathbf{f}(d \mathbf{u}),
$$


yielding

$$
\nabla\left(\frac{1}{|\mathbf{y}-\mathbf{r}|}\right)=|\mathbf{y}-\mathbf{r}|^{-3} \mathbf{f}^{t}(\mathbf{y}-\mathbf{r})
$$

and

$$
4 \pi \varphi_{s}(\mathbf{y}, t) / \epsilon=\int_{\Omega} \frac{\mathbf{f M}_{0}}{|\mathbf{y}-\mathbf{r}|^{3}} \cdot(\mathbf{y}-\mathbf{r}) d A+o(\epsilon) / \epsilon .
$$

A straightforward computation based on 2.43 generates the scaled self field in the surrounding space:

$$
4 \pi \mathbf{h}_{s}(\mathbf{y}, t) / \epsilon=\int_{\Omega} \mathbf{G}\left(\mathbf{f M}_{0}\right) d A(\mathbf{u})+o(\epsilon) / \epsilon, \quad \text { where } \quad \mathbf{G}=\frac{3}{|\mathbf{y}-\mathbf{r}|^{5}}(\mathbf{y}-\mathbf{r}) \otimes(\mathbf{y}-\mathbf{r})-\frac{1}{|\mathbf{y}-\mathbf{r}|^{3}} \mathbf{I}
$$

in which $\mathbf{r}(\mathbf{u}, t)$ is the membrane position field at time $t$. Thus, the leading-order model generates the dominant part of the self field in the surrounding space (which is of order $\epsilon$ ) $a$ posteriori.

\subsection{Loading}

Turning now to the loading, suppose the lateral surfaces are subjected to pressures $P^{ \pm}$. The applied tractions are

$$
\mathbf{p}_{a}^{ \pm}=\mp\left(P^{ \pm}\right)\left(\mathbf{F}^{*}\right)^{ \pm} \mathbf{k}
$$

and we assume that

$$
P^{ \pm}=\epsilon p^{ \pm}+o(\epsilon),
$$

where $p^{ \pm}$are of order unity. In this case $\mathbf{q}=\mathbf{0}$ and

$$
\mathbf{p}=\alpha(\Delta p) \mathbf{n}
$$

in 3.38, where $\Delta p=p^{-}-p^{+}$is the net lateral pressure across the membrane.

Invoking 3.33 and the foregoing thin-film approximations, we find that 3.5 reduces to

$$
\operatorname{Div}_{\|}\left(\mathbf{P}_{0} \mathbf{1}\right)+\alpha(\Delta p) \mathbf{n}+\mu_{0}\left(\operatorname{grad} \mathbf{h}_{a}\right)_{0} \mathbf{m}_{0}=\rho_{\kappa 0} \ddot{\mathbf{r}},
$$

to leading order, where $\left(\operatorname{gradh} \mathbf{h}_{a}\right)_{0}$ is evaluated using 2.36 and 2.40 with $\mathbf{y}$ replaced by $\mathbf{r}$, and

$$
\mathbf{P}_{0}=W_{\mathbf{F}}\left(\mathbf{F}_{0}, \mathbf{m}_{0}\right)-q_{0} \mathbf{F}_{0}^{-t} .
$$

This is augmented by the algebraic constraints $3.6_{2}$ and $3.38_{2}$. Using $3.9,3.44$, and 3.45 the leading-order constraints are found to be

$$
W_{\mathbf{m}}\left(\mathbf{F}_{0}, \mathbf{m}_{0}\right)=\mu_{0} \mathbf{h}_{a}(\mathbf{r}) \quad \text { and } \quad \mathbf{P}_{0} \mathbf{k}=\mathbf{0} .
$$


Together with 3.7, 3.11, 3.21, 3.34 and 3.48, these furnish a system for the determination of $\mathbf{r}(\mathbf{u}, t), \mathbf{e}(\mathbf{u}, t), \mathbf{M}_{0}(\mathbf{u}, t)\left(=\mathbf{1 M}_{0}\right)$ and $q_{0}(\mathbf{u}, t)$. In practice we solve the equation obtained by multiplying 3.47 through by $\epsilon$. This yields the equation of motion for the membrane, which in turn furnishes the leading-order approximation for a thin sheet. Our preference for 2.92 over 2.17 is due the availability of an explicit formula for the gradient of the applied field (cf. 2.40). From 3.7 and $3.49_{1}$ it is clear that the constraint 3.35 imposes a restriction, not only on the magnetization, but also on the deformation and director fields $\mathbf{r}$ and $\mathbf{e}$, and thus on the the geometry of the film in the presence of an applied field. In particular, this allows the orientation of the tangent plane to the membrane to adjust in response to the applied field.

The literature on magnetoelasticity in thin structures is typically based on an a priori constraint of the Kirchoff-Love type (i.e., $\mathbf{e}=\mathbf{0}$ ) on the director field (e.g. [41] ). However, in Chapter 6 below we find that solutions deviate substantially from Kirchhoff-Love kinematics. Because we have confined attention to states of magnetization that are optimal at any deformation, and thereby eliminated magnetization as an independent variable, it follows by relaxation of constraints by restrictions that the Kirchhoff-Love type impede the attainment of minima of the overall potential energy. Indeed, the analysis of [2] indicates that the Kirchhoff-Love constraint is generally incompatible with 3.35. Therefore the present model is optimal relative to formulations in which such constraints are imposed at the outset. Kirchhoff-Love kinematics are obtained if the effects of deformation and magnetization are uncoupled in the expression for the strain-energy function, as in weakly magnetized materials subjected to applied fields of sufficient intensity Chapter 5 [2].

Standard mixed traction/position problems consist in the specification of $\mathbf{r}$ and the traction

$$
\tau=\mathbf{P}_{0} \mathbf{1} \nu
$$

on complementary parts of the boundary curve $\partial \Omega$. Here $\tau$ is the value on $\partial \Omega$ of the exact traction field acting on a part of the cylindrical generating surface of the body where tractions are assigned. In this work we assume position to be prescribed on $\partial \Omega \times[-\epsilon / 2, \epsilon / 2]$ and thus assign $\mathbf{r}$ everywhere on $\partial \Omega$.

\subsection{Solvability of the Constraints}

We demonstrate the solvability of the constraints $3.49_{1,2}$ for $\mathbf{M}$ and $\mathbf{e}$ at a given deformation $\mathbf{r}(\mathbf{u}, t)$ of $\Omega$. To ease the notation, here and henceforth the subscript ${ }_{0}$ is suppressed on the understanding that all fields discussed are the restrictions to $\Omega$ of three-dimensional fields identified by the same symbol. We impose 3.34 at the outset, and thus find it more convenient to work with a formulation based on the use of $\mathbf{F}$ and $\mathbf{M}$, rather than $\mathbf{F}$ and $\mathbf{m}$, as independent variables. To this end we invoke bulk incompressibility and use 2.104 to define the function

$$
\tilde{W}(\mathbf{F}, \mathbf{M})=W(\mathbf{F}, \mathbf{F M}) \quad \text { for } \quad \mathbf{M} \in \Omega^{\prime} .
$$


Consider a one-parameter family of magnetizations $\mathbf{M}(u) \in \Omega^{\prime}$. Using a superposed dot to denote the derivative with respect to the parameter, we derive $\tilde{W}_{\mathbf{M}} \cdot \dot{\mathbf{M}}=W_{\mathbf{m}} \cdot \mathbf{F} \dot{\mathbf{M}}$ at fixed $\mathbf{F}$, and therefore

$$
\left[\tilde{W}_{\mathbf{M}}-\mathbf{F}^{t}\left(W_{\mathbf{m}}\right)\right] \cdot \dot{\mathbf{M}}=0 \quad \text { for all } \quad \dot{\mathbf{M}} \in \Omega^{\prime},
$$

wherein $\tilde{W}_{\mathbf{M}} \in \Omega^{\prime}$. It follows that $\tilde{W}_{\mathbf{M}}=\mathbf{1 F}^{t}\left(W_{\mathbf{m}}\right)$, where $\mathbf{1 F}^{t}=\mathbf{f}^{t}$ by virtue of 3.7 ; eq. 3.52 then implies that

$$
\tilde{W}_{\mathbf{M}}=\mu_{0} \mathbf{f}^{t} \mathbf{h}_{a}(\mathbf{r}) \text {. }
$$

We regard this as an equation for $\mathbf{M}$ in which $\mathbf{r}$, $\mathbf{f}$ and $\mathbf{e}$ (hence $\mathbf{F}$ ) are assigned. To investigate its solvability we compute another derivative, again at fixed $\mathbf{F}$, obtaining

$$
\left(\tilde{W}_{\mathbf{M M}}\right) \dot{\mathbf{M}}=\mathbf{f}^{t}\left(W_{\mathbf{m m}}\right) \dot{\mathbf{m}}=\left[\mathbf{f}^{t}\left(W_{\mathbf{m m}}\right) \mathbf{f}\right] \dot{\mathbf{M}} .
$$

Therefore,

$$
\dot{\mathbf{M}} \cdot\left(\tilde{W}_{\mathrm{MM}}\right) \dot{\mathbf{M}}=\mathbf{f} \dot{\mathbf{M}} \cdot\left(W_{\mathbf{m m}}\right) \mathbf{f} \dot{\mathbf{M}},
$$

which is positive for all non-zero $\dot{\mathbf{M}}$ by virtue of $2.116_{2}$. Accordingly, $\tilde{W}_{\mathbf{M M}}$ is positive definite and $\tilde{W}(\mathbf{F}, \cdot)$ is strictly convex. Equation 3.53 therefore possesses a unique solution $\hat{\mathbf{M}}$ which minimizes $\tilde{W}$ at fixed $\mathbf{F}$. This in turn determines the magnetization $\mathbf{m}=\mathbf{f} \hat{\mathbf{M}}$, which furnishes the unique solution to

$$
W_{\mathbf{m}}=\mu_{0} \mathbf{h}_{a}(\mathbf{r}) .
$$

Next, we fix $\mathbf{f}$ and define

$$
G(\mathbf{e}, \mathbf{m})=W(\mathbf{f}+\mathbf{d}(\mathbf{f}, \mathbf{e}) \otimes \mathbf{k}, \mathbf{m}),
$$

where $\mathbf{d}(\mathbf{f}, \mathbf{e})$ is the function defined by 3.11, in which $\alpha$ and $\mathbf{n}$ are determined by $\mathbf{f}$ via 3.12 and 3.14. Consider one-parameter families $\mathbf{e}(u)$ and $\mathbf{m}(u)$. The former induces the one-parameter family $\mathbf{F}(u)$ of deformation gradients with derivative $\dot{\mathbf{F}}=\mathbf{f} \dot{\mathbf{e}} \otimes \mathbf{k}$ (cf. 3.7 and 3.11). Accordingly,

$$
\dot{G}=\dot{\mathbf{e}} \cdot \mathbf{f}^{t}\left(W_{\mathbf{F}}\right) \mathbf{k}+\dot{\mathbf{m}} \cdot W_{\mathbf{m}}, \quad \text { yielding } \quad G_{\mathbf{e}}=\mathbf{f}^{t}\left(W_{\mathbf{F}}\right) \mathbf{k} \quad \text { and } \quad G_{\mathbf{m}}=W_{\mathbf{m}} .
$$

Using 3.48 and the invertibility of $\mathbf{F}^{t}$, we find the constraint $3.49_{2}$ to be equivalent to

$$
G_{\mathbf{e}}=\mathbf{0} \quad \text { and } \quad q=\mathbf{d} \cdot\left(W_{\mathbf{F}}\right) \mathbf{k} .
$$

To address the first of these equations, we keep $\mathbf{f}$ fixed and compute

$$
\left(G_{\mathbf{e}}\right)^{\cdot}=\mathbf{f}^{t}\left\{W_{\mathbf{F F}}[\mathbf{f} \dot{\mathbf{e}} \otimes \mathbf{k}]+\left(W_{\mathbf{F m}}\right) \dot{\mathbf{m}}\right\} \mathbf{k} .
$$


Here we regard $\mathbf{m}(u)$ as the magnetization induced by $\mathbf{e}(u)$ via 3.56 ; that is, we use the unique solution $\mathbf{M}=\hat{\mathbf{M}}(\mathbf{e})$ to 3.53 , associated with fixed $\mathbf{r}$ and $\mathbf{f}$, to generate $\mathbf{m}(u)=\mathbf{f} \hat{\mathbf{M}}(\mathbf{e}(u))$. This satisfies 3.56 identically for all $\mathbf{e}(u)$ with $u$ in some open interval. It follows that

$$
\dot{\mathbf{m}}=-\left(W_{\mathbf{m m}}\right)^{-1}\left(W_{\mathbf{m F}}\right)(\mathbf{f} \dot{\mathbf{e}} \otimes \mathbf{k}),
$$

so that 3.60 reduces to

$$
\left(G_{\mathbf{e}}\right)^{\cdot}=\mathbf{f}^{t}\{\mathbf{A}(\mathbf{k})\}(\mathbf{f} \dot{\mathbf{e}}),
$$

where $\mathbf{A}(\cdot)$ is defined by 2.117 .

With these results in hand, we define a function $\Gamma(\mathbf{e})$ by

$$
\Gamma(\mathbf{e})=G(\mathbf{e}, \mathbf{f} \hat{\mathbf{M}}(\mathbf{e}))-\mu_{0} \mathbf{h}_{a}(\mathbf{r}) \cdot \mathbf{f} \hat{\mathbf{M}}(\mathbf{e}),
$$

at the same $\mathbf{r}$ and $\mathbf{f}$. Inserting $\mathbf{e}(u)$ and evaluating the derivative, we find from 3.56 and $3.58_{2}$ that

$$
\dot{\Gamma}=G_{\mathbf{e}} \cdot \dot{\mathbf{e}}
$$

for all $u$ in some open interval. Then,

$$
\ddot{\Gamma}=\left(G_{\mathbf{e}}\right)^{\cdot} \cdot \dot{\mathbf{e}}+G_{\mathbf{e}} \cdot \ddot{\text { ë. }}
$$

The domain of $\Gamma(\cdot)$ is the linear space $\Omega^{\prime}$, a convex set. If $\mathbf{e}_{1,2}$ belong to this domain, then so do all points on the straight-line path

$$
\mathbf{e}(u)=u \mathbf{e}_{2}+(1-u) \mathbf{e}_{1} ; \quad u \in(0,1),
$$

on which 3.65 reduces to

$$
\ddot{\Gamma}=\mathbf{f} \dot{\mathrm{e}} \cdot\{\mathbf{A}(\mathbf{k})\}(\mathbf{f} \dot{\mathbf{e}}) ; \quad \dot{\mathbf{e}}=\mathbf{e}_{2}-\mathbf{e}_{1} \neq \mathbf{0} .
$$

Setting $\mathbf{a}=\mathbf{f} \dot{\mathbf{e}} \in T_{\omega}$ and $\mathbf{b}=\mathbf{k}$, we find that 2.118 is satisfied because $\mathbf{F}^{*} \mathbf{k}=\alpha \mathbf{n}$ is orthogonal to $T_{\omega}$ (cf. 3.12). Accordingly, the strong-ellipticity inequality $2.116_{1}$ is applicable and implies that $\ddot{\Gamma}>0$. Integration of this result over $(0, u)$ and then again over $(0,1)$ yields the conclusion that $\Gamma(\mathbf{e})$ is strictly convex; i.e.,

$$
\Gamma\left(\mathbf{e}_{2}\right)-\Gamma\left(\mathbf{e}_{1}\right)>\Gamma_{\mathbf{e}}\left(\mathbf{e}_{1}\right) \cdot\left(\mathbf{e}_{2}-\mathbf{e}_{1}\right)
$$

for all unequal pairs $\mathbf{e}_{1,2}$, wherein $\Gamma_{\mathbf{e}}=G_{\mathbf{e}}$ by virtue of 3.64 . Because strictly convex functions possess unique stationary points, we conclude that $3.59_{1}$ has a unique solution $\mathbf{e}^{*}$. In particular, this solution satisfies $\Gamma(\mathbf{e})>\Gamma\left(\mathbf{e}^{*}\right)$ for all $\mathbf{e} \neq \mathbf{e}^{*}$ and therefore furnishes the unique minimizer of $\Gamma(\mathbf{e})$. With this solution in hand, the unique magnetization field associated with a given deformation $\mathbf{r}(\mathbf{u}, t)$, and attendant gradient $\mathbf{f}$, is given by $\mathbf{m}=\mathbf{f} \hat{\mathbf{M}}\left(\mathbf{e}^{*}\right)$. 


\subsection{Lyapunov Functions}

We have shown that the constraints 3.53 and $3.59_{1}$ possess unique solutions e and $\mathbf{M}$ at fixed $\mathbf{r}$ and $\mathbf{f}$. To obtain them, use may be made of the Newton-Raphson method, for example. The convexity conditions established in the foregoing ensure that the associated iterates converge to a unique solution. Alternatively, we may embed 3.53 and $3.59_{1}$ into the artificial dynamical problems

$$
m_{m} \ddot{\mathbf{M}}+c_{m} \dot{\mathbf{M}}+\tilde{W}_{\mathbf{M}}=\mu_{0} \mathbf{f}^{t} \mathbf{h}_{a}(\mathbf{r}) \quad \text { and } \quad m_{e} \ddot{\mathbf{e}}+c_{e} \dot{\mathbf{e}}+G_{\mathbf{e}}=\mathbf{0},
$$

respectively, in which $m_{m}$ and $c_{m}$ are positive constants associated with magnetization and $m_{e}$ and $c_{e}$ are positive constants associated with the director and the superposed dots in the two equations now identify derivatives with respect to time-like parameters $\tau_{1,2}$, respectively. Equilibria of this system are precisely the unique solutions to 3.53 and $3.59_{1}$. Further, solutions to this system satisfy the energy balances

$$
\frac{d}{d \tau_{1}}\left(\frac{1}{2} m|\dot{\mathbf{M}}|^{2}+\tilde{W}\right)=-c|\dot{\mathbf{M}}|^{2} \quad \text { and } \quad \frac{d}{d \tau_{2}}\left(\frac{1}{2} m|\dot{\mathbf{e}}|^{2}+\Gamma\right)=-c|\dot{\mathbf{e}}|^{2} .
$$

Standard theory for ordinary differential equations then ensures the existence of trajectories of $3.69_{1,2}$ for arbitrary initial data on which

$$
L_{1}=\frac{1}{2} m|\dot{\mathbf{M}}|^{2}+\tilde{W} \quad \text { and } \quad L_{2}=\frac{1}{2} m|\dot{\mathbf{e}}|^{2}+\Gamma
$$

are strictly decreasing. Our results concerning the minimizing properties of equilibria then imply that $L_{1,2}$ furnish Lyapunov functions for $3.69_{1,2}$ respectively. All trajectories tend asymptotically to solutions of the constraints 3.53 and $3.59_{1}$, and these are stable equilibria of the dynamical system [22]. The implementation of these results is discussed in Chapter 4 .

Finally, we use the energy balance 2.112 to construct a Lyapunov function for the motion $\mathbf{r}(\mathbf{u}, t)$. To this end we observe, using 2.109, 2.110, 2.111, 2.113 and 2.114, that

$$
K=\epsilon K_{M}+o(\epsilon), \quad L=\epsilon L_{M}+o(\epsilon) \quad \text { and } \quad E=\epsilon E_{M}+o(\epsilon),
$$

where

$$
\begin{aligned}
K_{M} & =\frac{1}{2} \int_{\Omega} \rho_{\kappa}|\dot{\mathbf{r}}|^{2} d A, \quad L_{M}=\int^{V} p^{-}(v) d v-p^{+} V \\
\text { and } \quad E_{M} & =\int_{\Omega} W(\mathbf{F}, \mathbf{m}) d A-\mu_{0} \int_{\Omega} \mathbf{h}_{a}(\mathbf{r}) \cdot \mathbf{m} d A-L_{M},
\end{aligned}
$$

respectively, are the leading-order (membrane) approximations to the kinetic energy, pressure potential, and potential energy, in which

$$
V=\frac{1}{3} \int_{\Omega} \alpha \mathbf{n} \cdot \mathbf{r} d A
$$


is the volume of the compressible gas contained by the membrane. From the leading-order equation of motion 3.47 , we obtain

$$
\dot{K}_{M}=\int_{\Omega} \dot{\mathbf{r}} \cdot\left[\operatorname{Div}{ }_{\|}(\mathbf{P} \mathbf{1})+\alpha(\Delta p) \mathbf{n}+\mu_{0}\left(\operatorname{grad} \mathbf{h}_{a}\right) \mathbf{m}\right] d A .
$$

Using [34]

$$
\dot{L}_{M}=\int_{\Omega} \alpha(\Delta p) \mathbf{n} \cdot \dot{\mathbf{r}} d A
$$

this is reduced to

$$
\dot{K}_{M}=\dot{L}_{M}+\mu_{0} \int_{\Omega} \mathbf{m} \cdot \dot{\mathbf{h}}_{a} d A+\int_{\partial \Omega} \mathbf{P} 1 \nu \cdot \dot{\mathbf{r}} d S-\int_{\Omega} \mathbf{P} \mathbf{1} \cdot \dot{\mathbf{f}} d A,
$$

where we have used $\dot{\mathbf{h}}_{a}=\left(\operatorname{grad} \mathbf{h}_{a}\right) \dot{\mathbf{r}}$ for stationary applied fields, together with the symmetry of $\operatorname{grad} \mathbf{h}_{a}$. In this work we assume $\mathbf{r}$ to be fixed on $\partial \Omega$ and accordingly suppress the integral over $\partial \Omega$. We now use $3.49_{1}$ and combine the result with $3.73_{3}$ to derive

$$
\frac{d}{d t}\left(K_{M}+E_{M}\right)=\int_{\Omega}\left(W_{\mathbf{F}} \cdot \dot{\mathbf{F}}-\mathbf{P} \mathbf{1} \cdot \dot{\mathbf{f}}\right) d A .
$$

Using 3.48 with the constraint of bulk incompressibility in the form $\mathbf{F}^{-t} \cdot \dot{\mathbf{F}}=0$, together with 3.2 and 3.7 , we find that

$$
W_{\mathbf{F}} \cdot \dot{\mathbf{F}}=\mathbf{P} \cdot \dot{\mathbf{F}}=\mathbf{P} \mathbf{1} \cdot \dot{\mathbf{f}}+\mathbf{P k} \cdot \dot{\mathbf{d}}
$$

and thereby reduce 3.78 to

$$
\frac{d}{d t}\left(K_{M}+E_{M}\right)=\int_{\Omega} \mathbf{P k} \cdot \dot{\mathbf{d}} d A,
$$

which vanishes by virtue of 3.49. Thus the leading-order model yields the conservation law $\frac{d}{d t}\left(K_{M}+E_{M}\right)=0$, which is replaced, in the presence of dissipation, by the imbalance $\frac{d}{d t}\left(K_{M}+E_{M}\right) \leq 0$. This observation suggests that a dissipative numerical scheme may be based on a discretization of the artificial dynamical equation

$$
\operatorname{Div}_{\|}(\mathbf{P} \mathbf{1})+\alpha(\Delta p) \mathbf{n}+\mu_{0}\left(\operatorname{grad} \mathbf{h}_{a}\right) \mathbf{m}=\rho_{\kappa} \ddot{\mathbf{r}}+c \dot{\mathbf{r}},
$$

where $c$ is a suitable constant. It is straightforward to show that if this equation is used in place of 3.47 , then the leading-order energy balance is replaced by

$$
\frac{d}{d t}\left(K_{M}+E_{M}\right)=-c \int_{\Omega}|\dot{\mathbf{r}}|^{2} d A .
$$


Our earlier observation that stable equilibria minimize $E$ implies that $E_{M}$ is minimized, to leading order in thickness. Indeed, it is easily verified that 3.56 and the static specialization of 3.47 furnish the Euler-Lagrange equations for $E_{M}$. Consequently, $K_{M}+E_{M}$ decays on trajectories of 3.81, provided that $c>0$, and achieves a strict minimum at a stable equilibrium. It therefore yields a Lyapunov function for 3.81, whose equilibria coincide with those of 3.47. This conclusion applies strictly only to a finite-dimensional projection of the problem associated with a spatial discretization of the equations on $\Omega$. It also presumes that equilibria are minimizers of $E_{M}$. However here we have only imposed necessary conditions for a minimum of the energy. In particular, in the purely mechanical specialization of the theory it is known that these conditions are insufficient to preclude compressive stresses in equilibrium, which violate the Legendre-Hadamard necessary condition for minimizers of $E_{M}$ [29]. In such circumstances the existence of minimizers may be restored by replacing the membrane energy with a suitable relaxation [1], [13], [29], and [37], which excludes compressive stress a priori via the mechanism of fine-scale wrinkling. This is the subject of tension-field theory [35]. In this work we apply the theory to problems that do not exhibit wrinkling and therefore do not require the explicit relaxation.

We emphasize the fact that 3.81 does not describe the actual dynamics of the membrane. Rather, it is used here solely to expedite the computation of equilibria by embedding the equilibrium problem into an artificial (finite-dimensional) dynamical system whose equilibria coincide with those of the physical problem. As such, it furnishes a convenient regularization of the equations. The strictly dissipative nature of this system is a feature shared by actual equations of motion that account for dissipation through constitutive equations rather than through modification of the equation of motion. However, 3.81 proves more convenient for the purpose of generating equilibria because it allows the discrete equations associated with the temporal evolution to be decoupled, affording a more efficient solution procedure. This is discussed in the next section. 


\section{Chapter 4}

\section{Finite Difference Method}

In order to solve the equilibrium equations we use a finite difference based on Green's theorem for the $D i v_{\|}$term in 3.81. Then the system of equations (3.69 and 3.81) is solved using dynamic relaxation [40] where artificial mass, damping, and time is added to each equilibrium equation. The associated system of equations is solved explicitly and the equilibrium deformation is reached in the long time limit. This general method for solving $\operatorname{Div}_{\|}(\mathbf{P} \mathbf{1})=\mathbf{0}$ is described in [14] and [32]. We will outline the method here with the addition of the nonDiv $_{\|}$forcing term in equation 3.81, and the use of dynamic relaxation to solve equations 3.69 and 3.81. We will also introduce a more complex grid that is more effective for circular reference planes and modify the approach in [14] and [32] for the added complexity.

The reference plane $\Omega$ is covered by a grid (or grids) of cells of the kind in Figures 4.1 and 4.2. Nodes are represented with a pair of integers $(i, j)$. It is noted that each cell consists of four nodes and each node has three or four neighbors. The gray area in Figure 4.1 and 4.2 corresponding to the associated zone formed by its nearest neighbors. The center of each edge of each zone are represented with half integers and are referred to as cell-centered points where the cell is represented by the dashed area in Figure 4.1 and 4.2. Some quantities are identified with nodes and are called node-centered variables (eg deformed position, divergence of stress) and others are identified with zones called zonecentered variables (e.g. stress, reference magnetization, and unknown portion of director).

If $\sigma_{\alpha}$ is a piecewise differentiable field in the plane and $C$ is a closed contour with interior $D$, Green's theorem states that

$$
\int_{D} \frac{\partial \sigma_{\alpha}}{\partial x_{\alpha}} d A=e_{\alpha \beta} \oint_{C} \sigma_{\alpha} d x_{\beta}
$$

where $e_{\alpha \beta}$ is the two-dimensional alternator defined by $e_{12}=-e_{21}=1, \quad e_{11}=e_{21}=0$. For nodes with four neighbors a one-point integration rule is applied to the left side of the equation over the zone $D$, the gray region of Figure 4.1. Again the one-point integration rule is applied, but this time to the right side of the equation to the four edges of the path $C$, the path around $D$, see Figure 4.1. The path integral is then the sum of these four integrals 


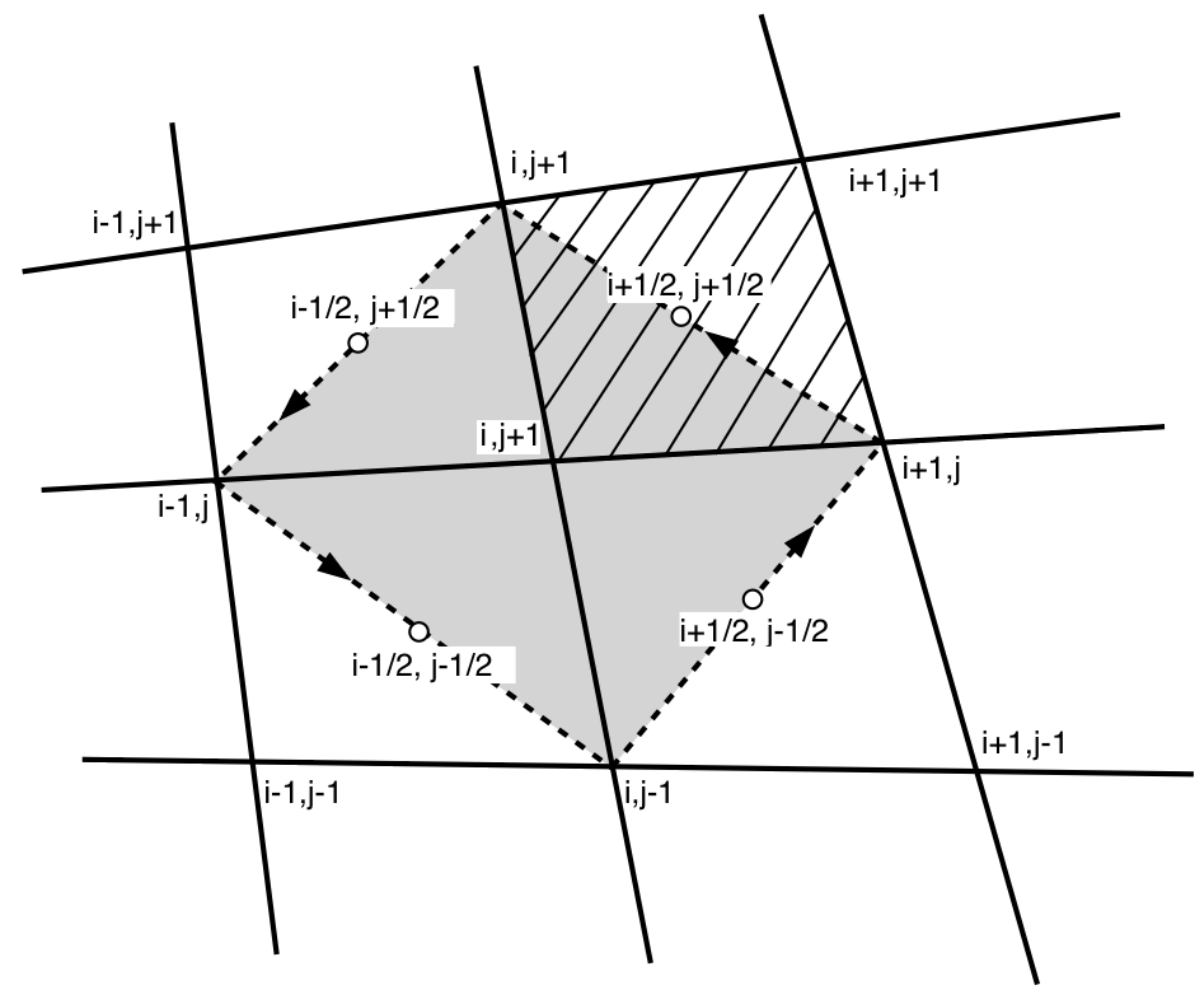

Figure 4.1: Unit cell of finite difference grid node with four neighboring nodes.

which yields the difference formula for a node with four neighbors:

$$
2 A^{i, j}\left(\sigma_{\alpha, \alpha}\right)^{i, j}=e_{\alpha \beta}\left(\begin{array}{c}
\sigma_{\alpha}^{i+\frac{1}{2}, j+\frac{1}{2}}\left(x_{\beta}^{i, j+1}-x_{\beta}^{i+1, j}\right)+ \\
\sigma_{\alpha}^{i-\frac{1}{2}, j+\frac{1}{2}}\left(x_{\beta}^{i-1, j}-x_{\beta}^{i, j+1}\right)+ \\
\sigma_{\alpha}^{i-\frac{1}{2}, j-\frac{1}{2}}\left(x_{\beta}^{i, j-1}-x_{\beta}^{i-1, j}\right)+ \\
\sigma_{\alpha}^{i+\frac{1}{2}, j-\frac{1}{2}}\left(x_{\beta}^{i+1, j}-x_{\beta}^{i, j-1}\right)
\end{array}\right)
$$

where $A^{i, j}$ is the area associated with the node $(i, j)$ and is half the area of the gray quadrilateral in Figure 4.1:

$$
A^{i, j}=\frac{1}{4}\left[\left(x_{2}^{i-1, j}-x_{2}^{i+1, j}\right)\left(x_{1}^{i, j+1}-x_{1}^{i, j-1}\right)-\left(x_{1}^{i-1, j}-x_{1}^{i+1, j}\right)\left(x_{2}^{i, j+1}-x_{2}^{i, j-1}\right)\right]
$$

The new grid used for circular reference surfaces consists of combining two grids of the above type. The first is the "center" and the second is the "outside", see Figure 4.2. The four nodes at the corner of the "center" grid need special attention as they have three neighboring nodes instead of four, as above. These nodes are circled in Figure 4.2, and for a close up see 


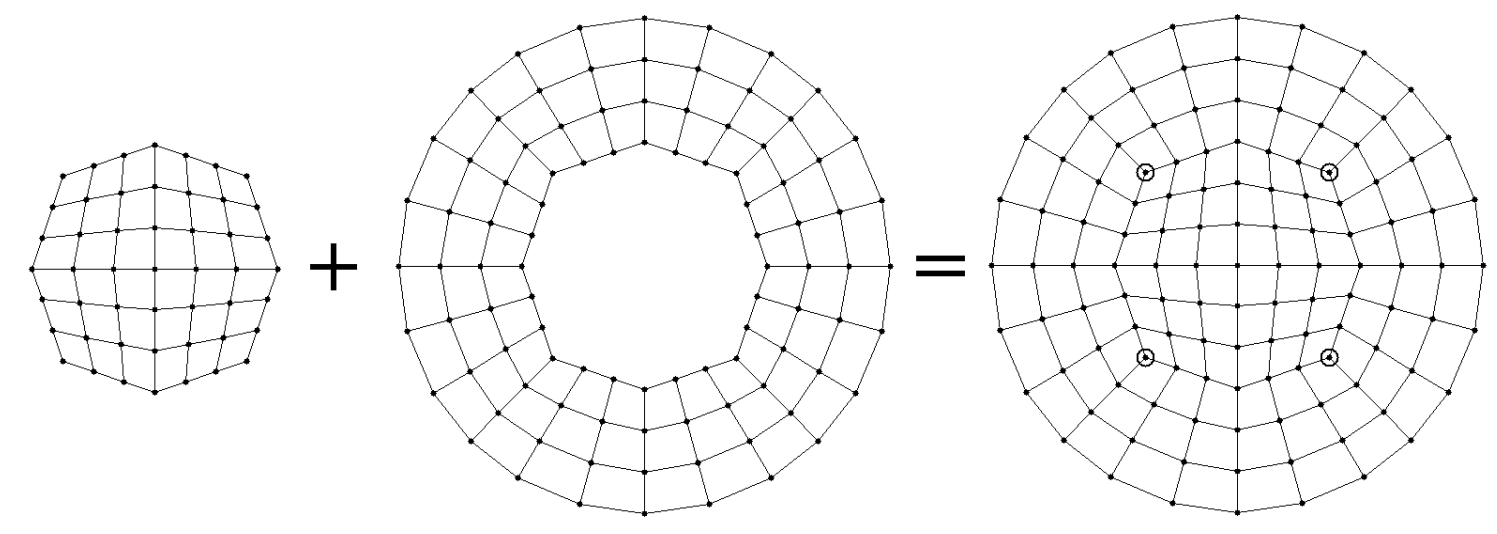

Figure 4.2: Butter fly grid, where the "center" grid + "edge" grid = full grid.

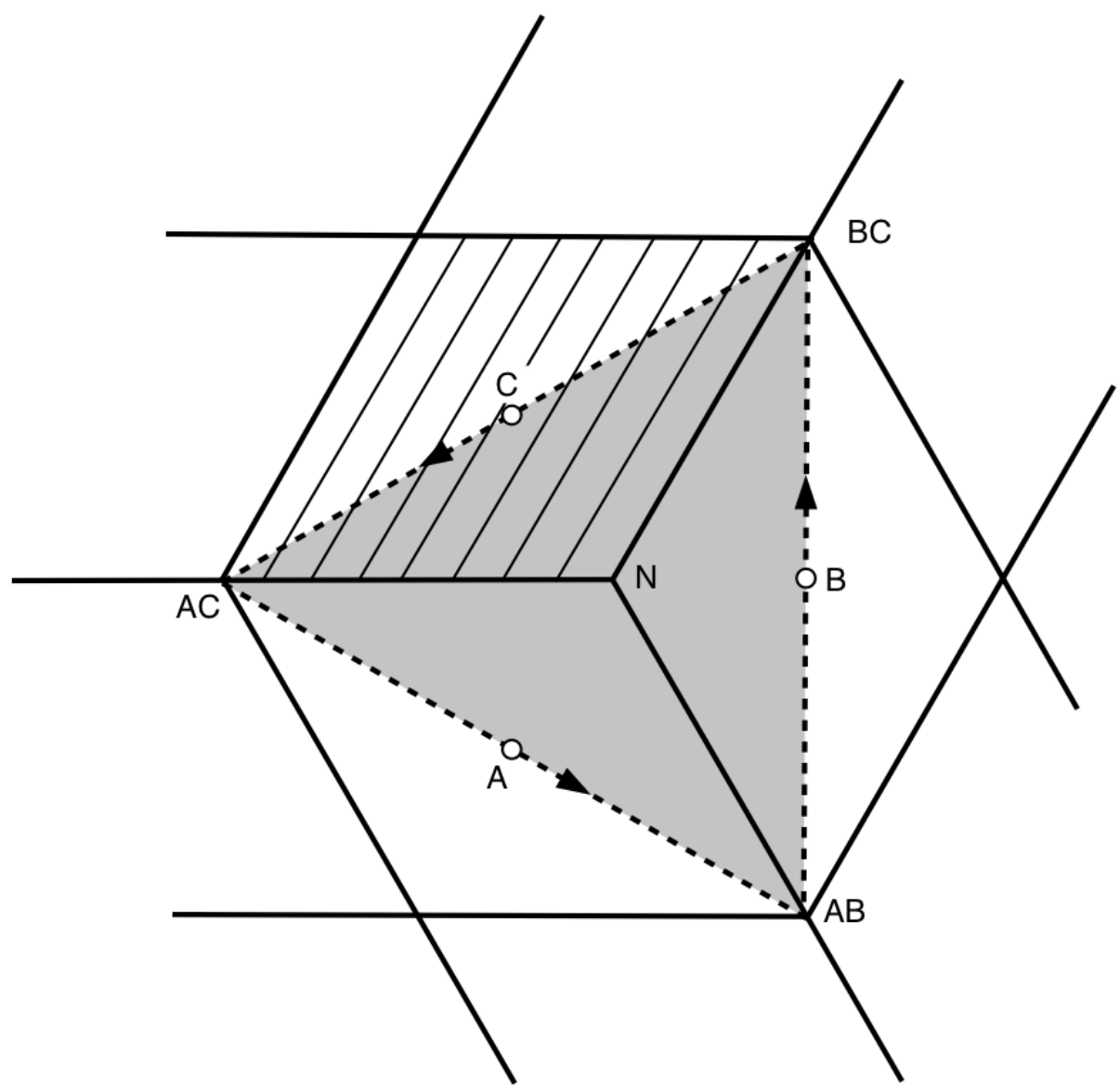

Figure 4.3: Close up of finite difference grid node with three neighboring nodes. 
Figure 4.3. The gray area in Figure 4.3 corresponds to the triangle associated with the corner nodes three nearest neighbors and forms a zone. The center of each edge of the triangle are represented with integers $(A, B, C)$ and can be represented by the appropriate cell centered nodes from the $\left(i+\frac{1}{2}, j+\frac{1}{2}\right)$ indexing. In the same manner as equations 4.2 were derived the equivalent equation for the corner nodes

$$
2 A^{N}\left(\sigma_{\alpha, \alpha}\right)^{N}=e_{\alpha \beta}\left(\sigma_{\alpha}^{A}\left(x_{\beta}^{A B}-x_{\beta}^{A C}\right)+\sigma_{\alpha}^{B}\left(x_{\beta}^{B C}-x_{\beta}^{A B}\right)+\sigma_{\alpha}^{C}\left(x_{\beta}^{A C}-x_{\beta}^{B C}\right)\right)
$$

where $A^{N}$ is the area associated with the node $(N)$ and is half the area of the gray triangle in Figure 4.3:

$$
A^{N}=\frac{1}{2}\left|\left(x_{1}^{A B}-x_{1}^{A C}\right)\left(x_{2}^{B C}-x_{2}^{A B}\right)-\left(x_{1}^{A B}-x_{1}^{B C}\right)\left(x_{2}^{A C}-x_{2}^{A B}\right)\right|
$$

For these corner nodes equations 4.4 and 4.5 are used instead of 4.3 and 4.2 where the node reference $(i, j)$ is replaced by $N$ to calculate the force due to the internal stress at these corner nodes.

It is also noted that nodes that fall on the intersection of the "center" grid and "edge" grid as well as common nodes from mapping of the "edge" need zone centered values from both the "center" and "edge" and different "edge" quantities where the simple adding and subtracting of 1 or $\frac{1}{2}$ to indices $i$ and $j$ does not work. The equations associated with these points are the same as the above mesh with care being taken on the numbering.

How fine the "butterfly" mesh is can be defined by two quantities $N_{c}$ and $N_{r} . N_{c}$ represents the number of nodes in the $\mathrm{x}$ and $\mathrm{y}$ direction of the "center" mesh, for Figure 4.2 $N_{c}=7 . N_{r}$ represents the number of radial nodes of the "edge", for Figure $4.2 N_{r}=3$, this is due to the fact that the inside nodes of the edge are the same as the outside of the center and will be represented by the center numbering.

The magnetoelastic equilibrium equation is given by 3.81 in which the right-hand side is suppressed. To facilitate its discretization, we exploit the fact that the term $\alpha \mathbf{n}$ associated with the pressure load may be expressed as a divergence on $\Omega$ [39]. Thus, $\mathbf{n}=n_{k} \mathbf{i}_{k}$, where $\mathbf{i}_{3}=\mathbf{k}$,

$$
\alpha n_{k}=\frac{1}{2} e_{i j k} e_{\alpha \beta} f_{i \alpha} f_{j \beta}=G_{k \beta, \beta},
$$

and

$$
G_{k \beta}=\frac{1}{2} e_{i j k} e_{\alpha \beta} f_{i \alpha} r_{j}
$$

in which $e_{i j k}$ and $e_{\alpha \beta}$ respectively are the three- and two-dimensional unit alternators $\left(e_{123}=\right.$ $e_{12}=+1$ ). This result is useful in the present circumstances because the net lateral pressure on the membrane is uniformly distributed. Thus, the equilibrium equation is equivalent to the system

$$
T_{k \alpha, \alpha}=R_{k}, \quad \text { where } \quad T_{k \alpha}=P_{k \alpha}+(\Delta p) G_{k \alpha} \quad \text { and } \quad R_{k}=-\mu_{0}\left(\operatorname{grad} \mathbf{h}_{a}\right)_{k i} f_{i \alpha} M_{\alpha} .
$$

Here $P_{k \alpha}=\mathbf{P} \cdot \mathbf{i}_{k} \otimes \mathbf{i}_{\alpha}$ are the components of $\mathbf{P} \mathbf{1},\left(\operatorname{grad} \mathbf{h}_{a}\right)_{k i}=\left(\partial h_{k}^{(a)}\right) /\left(\partial y_{i}\right) \cdot \mathbf{i}_{k} \otimes \mathbf{i}_{i}$ are the components of the gradient of the applied field (note that these can be solved directly 
from 2.40), $f_{k \alpha}=\mathbf{f} \cdot \mathbf{i}_{k} \otimes \mathbf{i}_{\alpha}=r_{k, \alpha}$ are the components of the surface deformation gradient, $r_{k}=\mathbf{i}_{k} \cdot \mathbf{r}$ are the Cartesian coordinates of a material point on the deformed surface and $M_{\alpha}=\mathbf{M} \cdot \mathbf{i}_{\alpha}$ are the magnetization components. $T_{k \alpha}$ in turn, depend on the magnetization, $r_{k}$ and the deformation gradient.

Equation $4.8_{1}$ is of the form

$$
\sigma_{\alpha, \alpha}=f
$$

where $\sigma_{\alpha}=T_{k \alpha}$ and $f=R_{k} ; k=1,2,3$. The $\sigma_{\alpha}$, in turn, depend on the magnetization and on the gradients of $\sigma=r_{k}(k=1,2,3)$.

To solve 4.9 for all nodes $(M=1,2, \ldots, N N-1, N N)$ we integrate over the region enclosed by the dashed quadrilateral of Figure 4.1 or the dashed triangle of Figure 4.2 for nodes with four or three neighbors, respectively where $N N$ is the total number of nodes, obtaining

$$
\Sigma^{M}=F^{M}
$$

where

$$
\Sigma^{M}=2 A^{M}\left(\sigma_{\alpha, \alpha}\right)^{M},
$$

and

$$
F^{M}=2 A^{M} f^{M} .
$$

In 4.11 the right-hand side is evaluated in terms of the zone-centered values of $\sigma_{\alpha}=T_{k \alpha}$ for $k=1,2,3$ via 4.2 or 4.4 . The latter are determined constitutively by corresponding zonecentered values of magnetization together with the current position and the deformation gradients. The current position is not stored at zone centered points but is calculated by averaging those of its four nearest neighbors. The deformation gradient is obtained at zone centered points by setting $\sigma_{\alpha}$ in equation 4.1 to the current position $\left(y_{k}\right)$ and applying the same approximations discussed above in determining $\sigma_{\alpha, \alpha}$, resulting in:

$$
2 A^{i+\frac{1}{2}, j+\frac{1}{2}}\left(f_{k \alpha}\right)^{i+\frac{1}{2}, j+\frac{1}{2}}=e_{\alpha \beta}\left(\begin{array}{c}
\left(x_{\beta}^{i, j+1}-x_{\beta}^{i+1, j}\right)\left(y_{k}^{i+1, j+1}-y_{k}^{i, j}\right) \\
-\left(x_{\beta}^{i+1, j+1}-x_{\beta}^{i, j}\right)\left(y_{k}^{i, j+1}-y_{k}^{i+1, j}\right)
\end{array}\right)
$$

where $A^{i+\frac{1}{2}, j+\frac{1}{2}}$ is the area associated with the zone, the dashed region of Figure 4.1, is:

$$
A^{i+\frac{1}{2}, j+\frac{1}{2}}=\frac{1}{2}\left[\left(x_{2}^{i, j+1}-x_{2}^{i+1, j}\right)\left(x_{1}^{i+1, j+1}-x_{1}^{i, j}\right)-\left(x_{1}^{i, j+1}-x_{1}^{i+1, j}\right)\left(x_{2}^{i+1, j+1}-x_{2}^{i, j}\right)\right]
$$

The scheme is seen to require one degree less differentiability than that required by the local differential equations. Discussions of the associated truncation errors are given by Hermann and Bertholf [15] and Silling [33] .

To solve equation 4.10, we introduce a regularization based on the artificial dynamical system (cf. 3.81)

$$
\Sigma^{M, n}=m^{M} \ddot{\sigma}^{M, n}+c^{M} \dot{\sigma}^{M, n}+F^{M, n}
$$


where $m^{M}=2 A^{M} \rho$ is the nodal mass, $c^{M}=2 A^{M} c$ is the nodal damping coefficient, $n$ is the time step, and superposed dots refer to derivatives with respect to (artificial) time. This is not the discrete form of the actual dynamical equations. Rather, it is an artificial system introduced solely to expedite the computation of equilibria. The basic method, known as dynamic relaxation [40], is a powerful tool for generating equilibria in a wide variety of nonlinear problems. It was developed for membrane theory in a purely mechanical setting in [13], [1] and [37] and extended to coupled thermoelasticity in [39].

We observe that the matrix $G_{k \beta}$ associated with lateral pressure is evaluated at zonecentered points (cf. 4.8 and 4.10). However, this involves the deformation $r_{k}$ (cf. 4.7), a nodal variable. The required evaluation is based on the average of the deformations of the adjacent nodes. Similarly, 4.8 requires nodal values of $f_{k \alpha} M_{\alpha}$, which are obtained by averaging values at the four adjacent zone-centered points.

In the case of volume-dependent pressure loading it is necessary to evaluate the volume enclosed by the deformed membrane and the plane $\Omega$. This is obtained from 3.74 in which $\alpha \mathbf{n} \cdot \mathbf{r}=\frac{1}{2} e_{i j k} e_{\alpha \beta} f_{i \alpha} f_{j \beta} r_{k}$. The domain is divided into zones - the shaded regions in Figure 1 and the integral over each is estimated as the zone-centered value of the integrand multiplied by the shaded area, given by 4.14 . Similarly, the scaled self field at a given position $\mathbf{y}$ in the surrounding space is obtained by using 3.43 , in which the integral is replaced by the sum of the integrals over the zones. Each of these is approximated by multiplying the value of the integrand at the relevant zone-centered point by the shaded area. The integrand is formed from zone-centered values of $\mathbf{f}$ and $\mathbf{M}$ and the averaged values of the nodal membrane position $\mathbf{r}$.

The time derivatives in 4.15 are approximated by the central differences

$$
\dot{\sigma}^{n}=\frac{1}{2}\left(\dot{\sigma}^{n+1 / 2}+\dot{\sigma}^{n-1 / 2}\right), \quad \ddot{\sigma}^{n}=\frac{1}{\Delta t}\left(\dot{\sigma}^{n+1 / 2}-\dot{\sigma}^{n-1 / 2}\right), \quad \dot{\sigma}^{n-1 / 2}=\frac{1}{\Delta t}\left(\sigma^{n}-\sigma^{n-1}\right),
$$

where $\Delta t$ is the time increment and the node label $(M)$ has been suppressed. Substitution into 4.15 furnishes the explicit, decoupled system

$$
\begin{aligned}
\left(\Delta t^{-1}+c / 2\right) m^{M} \dot{\sigma}^{M, n+1 / 2} & =\left(\Delta t^{-1}-c / 2\right) m^{M} \dot{\sigma}^{M, n-1 / 2}+\Sigma^{M, n}-F^{M, n}, \\
\sigma^{M, n+1} & =\sigma^{M, n}+\Delta t \dot{\sigma}^{M, n+1 / 2},
\end{aligned}
$$

which is used to advance the solution in time node-by-node.

The starting procedure is derived from the quiescent initial conditions

$$
\sigma^{M, 0}=\sigma_{0}\left(u_{\alpha}^{M}\right), \quad \dot{\sigma}^{M, 0}=0,
$$

where $\sigma_{0}\left(u_{\alpha}\right)$ is assigned. Thus, from 4.17 we obtain

$$
(2 / \Delta t) m^{M} \dot{\sigma}^{M, 1 / 2}=\Sigma^{M, 0}-F^{M, 0},
$$

in which the right-hand side is determined by the function $\sigma_{0}$. The solution is advanced to the first $t_{n}$ such that

$$
\max _{M}\left|\Sigma^{M, n}-F^{M, n}\right|<t o l
$$


where tol is a suitable tolerance. We remark that because only long-time limits of solutions are relevant, temporal accuracy is not an issue. Stability is addressed by using sufficiently small time steps selected on the basis of successive trials based on a sequence of values of $\Delta t$.

A similar temporal discretization is used to update the magnetization and director fields $\mathbf{M}$ and $\mathbf{e}$ at zone-centered points. Thus from 3.69 we arrive at:

$$
\begin{aligned}
&\left(\Delta \tau_{1}^{-1}+c_{m} / 2\right) m_{m}^{i+\frac{1}{2}, j+\frac{1}{2}} \dot{\mathbf{M}}^{i+\frac{1}{2}, j+\frac{1}{2}, n+\frac{1}{2}}=\left(\Delta \tau_{1}^{-1}-c_{m} / 2\right) m_{m}^{i+\frac{1}{2}, j+\frac{1}{2}} \dot{\mathbf{M}}^{i+\frac{1}{2}, j+\frac{1}{2}, n-\frac{1}{2}} \\
&\left.+\left(\mathbf{f}^{i+\frac{1}{2}, j+\frac{1}{2}, n}\right)^{-1} \mathbf{h}_{a}^{i+\frac{1}{2}, j+\frac{1}{2}, n}-\tilde{W}_{\mathbf{M}}^{i+\frac{1}{2}, j+\frac{1}{2}, n}\right) \\
& \mathbf{M}^{i+\frac{1}{2}, j+\frac{1}{2}, n+1}=\mathbf{M}^{i+\frac{1}{2}, j+\frac{1}{2}, n}+\Delta \tau_{1} \dot{\mathbf{M}}^{i+\frac{1}{2}, j+\frac{1}{2}, n+\frac{1}{2}} \\
&\left(\Delta \tau_{2}^{-1}+c_{e} / 2\right) m_{e}^{i+\frac{1}{2}, j+\frac{1}{2}} \dot{\mathbf{e}}^{i+\frac{1}{2}, j+\frac{1}{2}, n+\frac{1}{2}}=\left(\Delta \tau_{2}^{-1}-c_{e} / 2\right) m_{e}^{i+\frac{1}{2}, j+\frac{1}{2}} \dot{\mathbf{e}}^{i+\frac{1}{2}, j+\frac{1}{2}, n-\frac{1}{2}} \\
&+G_{\mathbf{e}}^{i+\frac{1}{2}, j+\frac{1}{2}, n} \\
& \\
& \mathbf{e}^{i+\frac{1}{2}, j+\frac{1}{2}, n+1}=\mathbf{e}^{i+\frac{1}{2}, j+\frac{1}{2}, n}+\Delta \tau_{2} \dot{\mathbf{e}}^{i+\frac{1}{2}, j+\frac{1}{2}, n+\frac{1}{2}}
\end{aligned}
$$

where

$$
\begin{gathered}
\mathbf{h}_{a}^{i+\frac{1}{2}, j+\frac{1}{2}, n}=\mathbf{h}_{a}\left(y^{i+\frac{1}{2}, j+\frac{1}{2}, n}\right), \\
\tilde{W}_{\mathbf{M}}^{i+\frac{1}{2}, j+\frac{1}{2}, n}=\tilde{W}_{\mathbf{M}}\left(\mathbf{f}^{i+\frac{1}{2}, j+\frac{1}{2}, n}, \mathbf{M}^{i+\frac{1}{2}, j+\frac{1}{2}, n}\right),
\end{gathered}
$$

$m_{m}^{i+\frac{1}{2}, j+\frac{1}{2}}$ is the nodal artificial mass associated with the magnetic equation, $c_{m}^{i+\frac{1}{2}, j+\frac{1}{2}}=$ $c_{m} m_{m}^{i+\frac{1}{2}, j+\frac{1}{2}}$ is the artificial damping coefficient associated with the magnetic equation, $\Delta \tau_{1}$ is the artificial time step associated with the magnetization, $m_{e}^{i+\frac{1}{2}, j+\frac{1}{2}}$ is the nodal artificial mass associated with the director equation, $c_{e}^{i+\frac{1}{2}, j+\frac{1}{2}}=c_{e} m_{e}^{i+\frac{1}{2}, j+\frac{1}{2}}$ is the artificial damping coefficient associated with the director equation, $\Delta \tau_{2}$ is the artificial time step associated with the director.

Consistency with the derivation of the Lyapunov functions $L_{1,2}$ of Section 3.6 requires the use of a staggered scheme in which the predicted position field at time step $n+1$ is fixed while integrating 3.70. We then start the integration of $3.70_{2}$ using the value of $\mathbf{e}$ at step $n$ as the initial condition (with the initial value $\dot{\mathbf{e}}=\mathbf{0}$ ). This calculation proceeds in increments of the time-like variable $\tau_{2}$. We fix the predicted value of $\mathbf{e}$ at the subsequent step and use this value to integrate $3.70_{1}$ with respect to $\tau_{1}$, using the value of $\mathbf{M}$ generated by the previous value of $\mathbf{e}$ as the initial condition (with initial value $\dot{\mathbf{M}}=\mathbf{0}$ ). This continues until convergence is achieved, yielding the magnetization associated with the predicted value of $\mathbf{e}$. The integration with respect to $\tau_{2}$ then resumes and the cycle is repeated until convergence 
is achieved, yielding the values of $\mathbf{e}$ and $\mathbf{M}$ associated with the position field at step $n+1$. The process is repeated until the deformation field converges, yielding the final equilibrium position, magnetization and director fields over all nodes and zone-centered points. However, numerical experiments indicate that this computationally intensive double-staggered scheme is not required in practice. Instead, we find that equilibrium states may be achieved by treating all fields on an equal basis as far as temporal integration is concerned.

The magnetization at step $n=0$ is set to zero. This is the unique solution to (112) if the applied field vanishes. Accordingly, the applied field intensity is first set to a small value and the equilibrium fields are obtained by the foregoing procedure. Successive equilibria are then computed for a sequence of increasing field intensities, using the equilibria associated with each member of the sequence as initial values for the next member.

In a similar manner as with the equation of motion we assume the time derivative of magnetization and unknown portion of the director to be zero $\left(\dot{\mathbf{M}}^{i+\frac{1}{2}, j+\frac{1}{2}, 0}=\mathbf{0}\right.$ and $\left.\dot{\mathbf{e}}^{i+\frac{1}{2}, j+\frac{1}{2}, 0}=\mathbf{0}\right)$ thus arrive at the time derivative equations for the first time step:

$$
\begin{gathered}
\left.\left(2 / \Delta \tau_{1}\right) m_{m}^{i+\frac{1}{2}, j+\frac{1}{2}} \dot{\mathbf{M}}^{i+\frac{1}{2}, j+\frac{1}{2}, \frac{1}{2}}=\left(\mathbf{f}^{i+\frac{1}{2}, j+\frac{1}{2}, n}\right)^{-1} \mathbf{h}_{a}^{i+\frac{1}{2}, j+\frac{1}{2}, n}-\tilde{W}_{\mathbf{M}}^{i+\frac{1}{2}, j+\frac{1}{2}, n}\right) \\
\left(2 / \Delta \tau_{2}\right) m_{d}^{i+\frac{1}{2}, j+\frac{1}{2}} \dot{\mathbf{e}}^{i+\frac{1}{2}, j+\frac{1}{2}, \frac{1}{2}}=G_{\mathbf{e}}^{i+\frac{1}{2}, j+\frac{1}{2}, n}
\end{gathered}
$$




\section{Chapter 5}

\section{Weakly Magnetized Material}

In this chapter we will discuss the first material model we consider, of weak magnetization. This material model will then be used to solve for the equilibrium deformation using a shooting method in Section 5.1, finite element method in Section 5.3 and finite difference method in Section 5.4. An instability is inferred from the shooting method and further investigated with the use of a variational method (Section 5.2). The study of the weak magnetized material and solving for equilibrium deformation has been published previously in $[2]$.

The limit of weak magnetization defined is defined by

$$
\mathbf{m}=\delta \overline{\mathbf{m}},
$$

where $|\delta| \ll 1$ and $|\overline{\mathbf{m}}|$ and $|\operatorname{grad} \overline{\mathbf{m}}|$ are of order $O(1)$ after suitable non-dimensionalization. When referring to estimates of this kind we intend that they apply to suitably non-dimensionalized variables. We also assume the applied magnetic field to be strong, in the sense that $\left|\mathbf{h}_{a}\right|=O\left(\delta^{-1}\right)$ and $\left|\operatorname{grad} \mathbf{h}_{a}\right|=O\left(\delta^{-1}\right)$ in $R$. Our assumptions imply that $\left|\mathbf{h}_{s}\right|=O(\delta)$ in $R$.

It follows from 3.56 and 5.1 that $\left|W_{\mathbf{m}}\right|=O\left(\delta^{-1}\right)$ whereas $|\mathbf{m}|=O(\delta)$. This is associated with behavior that is coercive in the sense that a large field is required to produce a small magnetic response. In this regime it is consistent to use the estimate

$$
W(\mathbf{F}, \mathbf{m})=W(\mathbf{F}, \mathbf{0})+\mathbf{m} \cdot W_{\mathbf{m}}(\mathbf{F}, \mathbf{0})+\frac{1}{2} \mathbf{m} \otimes \mathbf{m} \cdot W_{\mathbf{m m}}(\mathbf{F}, \mathbf{0})+o\left(|\mathbf{m}|^{2}\right) .
$$

It is shown below that $W(\mathbf{F}, \mathbf{m})=W(\mathbf{F},-\mathbf{m})$ for materials that are isotropic with a center of symmetry. For unsteady fields, this also follows from the requirement that $W(\mathbf{F}, \mathbf{m})$ be invariant under time reversals [24] (p. 137). A necessary and sufficient condition is that $W(\mathbf{F}, \mathbf{m})=\bar{W}(\mathbf{F}, \pi)$, where $\pi=\mathbf{m} \otimes \mathbf{m}[34]$. Straightforward calculation then yields

$$
W_{\mathbf{m}}=2\left(\bar{W}_{\pi}\right) \mathbf{m}
$$


implying that $W_{\mathbf{m}}(\mathbf{F}, \mathbf{0})=\mathbf{0}$ and

$$
W(\mathbf{F}, \mathbf{m})=W(\mathbf{F}, \mathbf{0})+\frac{1}{2} \mathbf{m} \otimes \mathbf{m} \cdot W_{\mathbf{m m}}(\mathbf{F}, \mathbf{0})+o\left(|\mathbf{m}|^{2}\right) .
$$

Accordingly, the relevant expression for the energy per unit reference volume is

$$
W(\mathbf{F}, \mathbf{m}) \simeq \hat{W}(\mathbf{F})+\frac{1}{2} \mathbf{m} \otimes \mathbf{m} \cdot \mathbf{A}(\mathbf{F}),
$$

where $\hat{W}(\mathbf{F})=W(\mathbf{F}, \mathbf{0})$ is the conventional strain-energy function associated with the purely mechanical theory and $\mathbf{A}(\mathbf{F})=W_{\mathbf{m m}}(\mathbf{F}, \mathbf{0})$. Given the paucity of empirical data, we assume, both for simplicity and for purposes of illustration, that

$$
\mathbf{A}(\mathbf{F})=A \mathbf{I},
$$

where $A$ is a fixed constant. This is shown below to be consistent with our assumption of isotropy. Accordingly, the expression for the energy becomes

$$
W(\mathbf{F}, \mathbf{m}) \simeq \hat{W}(\mathbf{F})+\frac{1}{2} A|\mathbf{m}|^{2} .
$$

From 3.56 and 5.7 we arrive at:

$$
\mathbf{m}=\frac{\mu_{0}}{A} \mathbf{h}_{a}
$$

If the relation between the magnetization and magnetic field is in the linear range (i.e. not close to saturation), then 5.8 can be compared to

$$
\mathbf{m}=\bar{\chi} \mathbf{h}_{a}
$$

where $\bar{\chi}$ is the magnetic susceptibility per unit volume of the membrane [17]. This correspondence establishes the connection:

$$
A=\frac{\mu_{0}}{\bar{\chi}}
$$

and finally the constitutive relation 5.7 in terms of standard material properties:

$$
W(\mathbf{F}, \mathbf{m}) \simeq \hat{W}(\mathbf{F})+\frac{\mu_{0}}{2 \bar{\chi}}|\mathbf{m}|^{2} .
$$

We will use the Mooney-Rivlin form of the conventional strain-energy function:

$$
\hat{W}(\mathbf{F})=w^{*}\left(\lambda_{1}, \lambda_{2}, \lambda_{3}\right)=\frac{G}{2}\left[\delta\left(\lambda_{1}^{2}+\lambda_{2}^{2}+\lambda_{3}^{2}-3\right)+(1-\delta)\left(\lambda_{1}^{-2}+\lambda_{2}^{-2}+\lambda_{3}^{-2}-3\right)\right]
$$

where $\lambda_{i}$ are the principal stretches, the positive square roots of the eigenvalues of the right Cauchy-Green tensor $\left(\mathbf{C}=\mathbf{F}^{t} \mathbf{F}\right)$ and $\delta \in(0,1]$ is a fixed parameter that is fitted to material 
data and $G$ is the (positive) ground-state shear modulus. The stretches are the coefficients in the representation [25]

$$
\mathbf{F}=\sum_{i=1}^{3} \lambda_{i} \mathbf{v}_{i} \otimes \mathbf{u}_{i}
$$

where $\mathbf{u}_{i}$ and $\mathbf{v}_{i}$ are the principal vectors of $\mathbf{F}^{t} \mathbf{F}$ and $\mathbf{F} \mathbf{F}^{t}$, respectively.

For this strain-energy function the necessity to solve for the magnetization using dynamic relaxation can be eliminated and solved directly from the applied magnetic field using equation 5.9. By combining $3.58_{2}$ and $3.59_{1}$ we can derive an algebraic problem for the elimination of $\mathbf{e}$. Using the fact that the deformation and magnetization are uncoupled $\left(W_{\mathbf{F m}}=\mathbf{0}\right)$ for this strain-energy function and strong ellipticity of the three-dimensional strain-energy function defined by 5.12 , it has been shown in [38] that the unique solution is given by $\mathbf{e}=\mathbf{0}$. It follows [38] that $\mathbf{u}_{3}= \pm \mathbf{k}$ and $\mathbf{u}_{1}, \mathbf{u}_{2} \in \Omega^{\prime}$. Further, from 3.10 and 3.11 we derive

$$
\mathbf{d}=\lambda \mathbf{n}, \text { where } \lambda=\left(\lambda_{1} \lambda_{2}\right)^{-1} \text {. }
$$

It follows from Equations $3.58_{2}$ and $3.59_{1}$ that the constraint pressure is [38]

$$
q=\lambda_{3} \frac{\partial w^{*}}{\partial \lambda_{3}}
$$

Let

$$
w\left(\lambda_{1}, \lambda_{2}\right)=w^{*}\left(\lambda_{1}, \lambda_{2}, \lambda_{3}\right) \quad \text { with } \quad \lambda_{3}=\left(\lambda_{1} \lambda_{2}\right)^{-1} .
$$

Then with 5.15 we have

$$
\frac{\partial w}{\partial \lambda_{\alpha}}=\frac{\partial w^{*}}{\partial \lambda_{\alpha}}-\frac{q}{\lambda_{\alpha}}
$$

Moreover, we may proceed from $3.79,5.13,5.17$, as in [24], to show that

$$
\begin{gathered}
\mathbf{f}=\sum_{\alpha=1}^{2} \lambda_{\alpha} \mathbf{v}_{\alpha} \otimes \mathbf{u}_{\alpha} \\
\mathbf{P} \mathbf{1}=\sum_{\alpha=1}^{2} \frac{\partial w}{\partial \lambda_{\alpha}} \mathbf{v}_{\alpha} \otimes \mathbf{u}_{\alpha}
\end{gathered}
$$

where $\mathbf{v}_{\alpha} \in T_{\omega(\mathbf{u})}$.

\subsection{Shooting Method}

We consider axisymmetric deformations $\mathbf{u} \mapsto \mathbf{r}(\mathbf{u})$, where $\mathbf{u}=r \mathbf{e}_{r}(\theta)$ and

$$
\mathbf{r}(\mathbf{u})=u(r) \mathbf{e}_{r}(\theta)+z(r) \mathbf{k},
$$


in which $r \in\left[0, r_{a}\right]$ is the radius from the axis of symmetry of a material point in the reference configuration; $\theta \in[0,2 \pi)$ is its azimuthal angle; $\mathbf{e}_{r}(\theta)=\mathbf{u} / r$ is the radial unit vector at azimuth $\theta ; \mathbf{k}$ is a fixed unit vector aligned with the symmetry axis; and $u, z$ are the radial and axial coordinates of the material point after deformation. This deformation maps a plane circular disc to a surface of revolution. The associated deformation gradient is

$$
\mathbf{f}=\left[u^{\prime}(r) \mathbf{e}_{r}+z^{\prime}(r) \mathbf{k}\right] \otimes \mathbf{e}_{r}+(u / r) \mathbf{e}_{\theta} \otimes \mathbf{e}_{\theta},
$$

where $(\cdot)^{\prime}=d(\cdot) / d r$ and $\mathbf{e}_{\theta}=\mathbf{k} \times \mathbf{e}_{r}=\mathbf{e}_{r}^{\prime}(\theta)$. The azimuthal and radial principal stretches are

$$
\begin{gathered}
\lambda_{1}:=\lambda=u(r) / r \\
\lambda_{2}:=\mu=\sqrt{\left(u^{\prime}\right)^{2}+\left(z^{\prime}\right)^{2},}
\end{gathered}
$$

and the corresponding principal axes are

$$
\mathbf{u}_{1}=\mathbf{v}_{1}=\mathbf{e}_{\theta}
$$

and

$$
\mathbf{u}_{2}=-\mathbf{e}_{r}, \quad \mathbf{v}_{2}=-\cos \phi(r) \mathbf{e}_{r}+\sin \phi(r) \mathbf{k},
$$

where (see Fig. 1)

$$
\cos \phi=u^{\prime} / \mu
$$

and

$$
\sin \phi=-z^{\prime} / \mu .
$$

Using 5.19 with formulae developed in [25], it is straightforward to show that

$$
\operatorname{Div}_{\|}(\mathbf{P} \mathbf{1})=r^{-1}\left[\left(w_{\lambda} \mathbf{v}_{1}\right)_{\theta}-\left(r w_{\mu} \mathbf{v}_{2}\right)_{r}\right],
$$

where Greek subscripts are used to denote partial derivatives. Thus,

$$
\operatorname{Div}_{\|}(\mathbf{P} \mathbf{1})=r^{-1}\left\{\left[w_{\lambda} \cos \phi-\left(r w_{\mu}\right)^{\prime}\right] \mathbf{v}_{2}-\left[w_{\lambda} \sin \phi+r w_{\mu} \phi^{\prime}\right] \mathbf{n}\right\},
$$

where

$$
\mathbf{n}=\cos \phi \mathbf{k}+\sin \phi \mathbf{e}_{r}
$$

is the unit normal to the deformed surface and we have used $\mathbf{v}_{2}^{\prime}=\phi^{\prime} \mathbf{n}$ with

$$
\mathbf{e}_{r}=-\cos \phi \mathbf{v}_{2}+\sin \phi \mathbf{n} .
$$

On combining 5.9 and 3.81 we find the contribution of the magnetic field to the equilibrium equation to be

$$
\mathbf{g}:=\mu_{0}\left(\operatorname{grad} \mathbf{h}_{a}\right) \mathbf{m}=\mu_{0} \bar{\chi}\left(\operatorname{grad} \mathbf{h}_{a}\right) \mathbf{h}_{a} .
$$




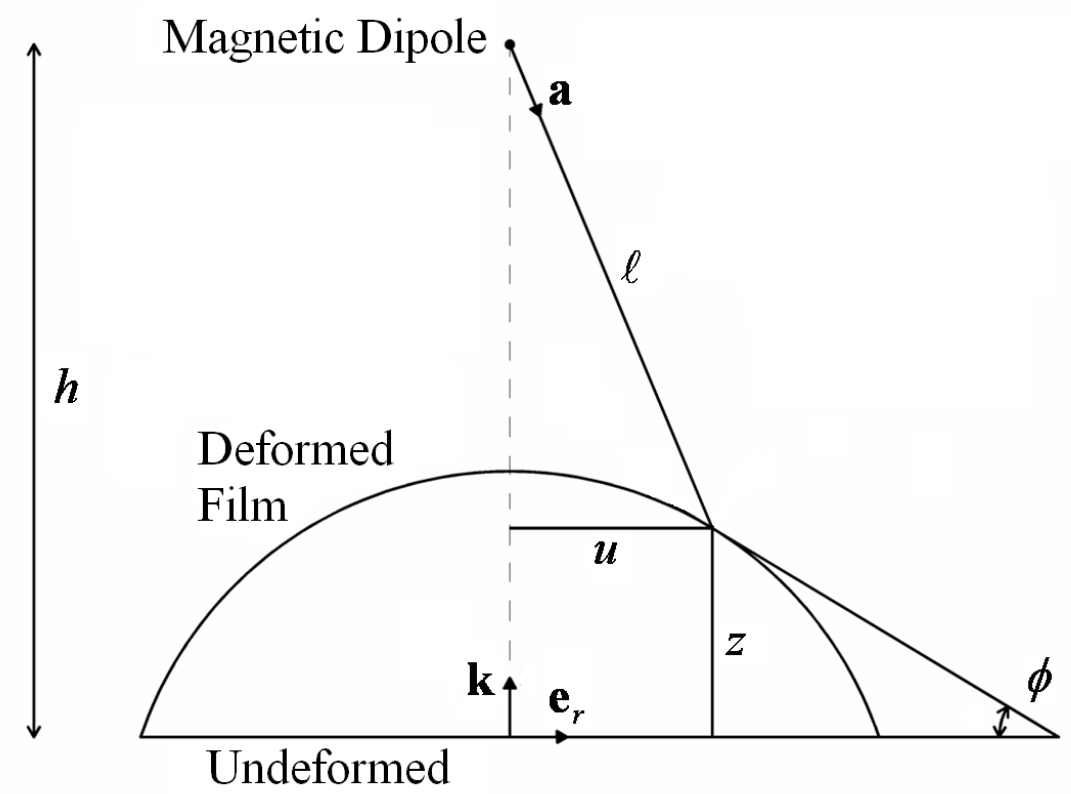

Film

Figure 5.1: Geometry for shooting method

After some calculation based on 2.35 and 2.40 this reduces to

$$
\mathbf{g}=\frac{H}{\ell^{8}}\left\{(\ell \mathbf{a} \cdot \mathbf{k}) \mathbf{k}-\left[1+4(\mathbf{a} \cdot \mathbf{k})^{2}\right](\ell \mathbf{a})\right\},
$$

where

$$
H:=3 D^{2} \mu_{0} \bar{\chi} .
$$

This is to be evaluated on the deformed membrane midsurface, where $\mathbf{y}=\mathbf{r}(\mathbf{u})$. Thus with a dipole position $\mathbf{y}_{d}=z_{d} \mathbf{k}$,

$$
\ell \mathbf{a}=u \mathbf{e}_{r}+\left(z-z_{d}\right) \mathbf{k}, \quad \ell^{2}=u^{2}+\left(z_{d}-z\right)^{2} .
$$

Use of $\alpha=\lambda \mu$ in the pressure term in 3.81 and projection of the resulting equation onto the unit tangent and normal to the deformed membrane furnish the coupled equilibrium equations

$$
w_{\lambda} \cos \phi-\left(r w_{\mu}\right)^{\prime}+r \mathbf{v}_{2} \cdot \mathbf{g}=0
$$

and

$$
\Delta p \lambda \mu-r w_{\mu} \phi^{\prime}-w_{\lambda} \sin \phi+r \mathbf{n} \cdot \mathbf{g}=0,
$$

where, from 5.24, 5.25 and 5.33,

$$
\mathbf{v}_{2} \cdot \mathbf{g}=\frac{H}{\ell^{8}}\left\{4\left(z_{d}-z\right) \frac{\left(z_{d}-z\right)^{2}}{\ell^{2}} \sin \phi+\left[1+4 \frac{\left(z_{d}-z\right)^{2}}{\ell^{2}}\right] u \cos \phi\right\},
$$


and

$$
\mathbf{n} \cdot \mathbf{g}=\frac{H}{\ell^{8}}\left\{4\left(z_{d}-z\right) \frac{\left(z_{d}-z\right)^{2}}{\ell^{2}} \cos \phi-\left[1+4 \frac{\left(z_{d}-z\right)^{2}}{\ell^{2}}\right] u \sin \phi\right\} .
$$

Equations 5.36 and 5.37 are further reduced by using the chain rule in 5.37. Thus,

$$
\mu^{\prime}=\left(r w_{\mu \mu}\right)^{-1}\left[w_{\lambda} \cos \phi-\hat{w}_{\mu}-w_{\lambda \mu}(\mu \cos \phi-u / r)+r \mathbf{v}_{2} \cdot \mathbf{g}\right]
$$

and

$$
\phi^{\prime}=\left(r w_{\mu}\right)^{-1}\left[\Delta p u \mu-w_{\lambda} \sin \phi+r \mathbf{n} \cdot \mathbf{g}\right]
$$

which, when combined with 5.24, 5.25, 5.26 and 5.27, completes a first-order system for the determination of the equilibrium deformation.

The foregoing system is integrated subject to the boundary conditions

$$
\begin{gathered}
u(0)=0, \\
\phi(0)=0, \\
\lambda\left(r_{a}\right)=\Lambda, \\
z\left(r_{a}\right)=0 .
\end{gathered}
$$

where $r_{a}$ is the outer radius of the film and according to 5.24 the third of these entails the assignment of radial position at the outer radius. Thus, we seek a continuously differentiable deformation field meeting prescribed position data everywhere on the boundary.

The equations are solved by a shooting method based on standard Euler forward differencing with a suitable mesh spacing $\Delta r$. The implementation of this method is straightforward after resolution of the singularities in 5.40 and 5.41 at $r=0$. To this end we assume that $\mu(r)$ is differentiable and Taylor-expand $r \mu$ to obtain $\mu(\Delta r)=\mu_{0}+\left(r \mu^{\prime}\right)_{0}$ to leading order, where the subscript 0 is now used to identify function values at $r=0$ and $\left(r \mu^{\prime}\right)_{0}$ is obtained from 5.40 in which $\cos \phi_{0}=1$ by virtue of 5.43 . Equations 5.26 and 5.42 imply that $u(r)=r \mu_{0}+o(r)$ near the origin and thus that $\lambda(0)=\mu(0)=\mu_{0}$. Taken together these furnish the leading-order estimate

$$
\mu(\Delta r)-\mu_{0}=\left(w_{\mu \mu}\right)^{-1}\left(w_{\lambda}-w_{\mu}\right)
$$

where the right-hand side is evaluated at $r=0$; this vanishes because of the isotropy of the material and the equibiaxial stretch at the origin. Therefore $\mu(\Delta r)=\mu_{0}$ and $u(\Delta r)=\mu_{0} \Delta r$ to the order of accuracy consistent with the Euler method. In the same way 5.41 and 5.43 are used in the Taylor expansion of $r \phi$ near the origin to obtain $\phi(\Delta r)=0$ to leading order. These estimates are used to start the integration of the equations at $r=\Delta r$. The shooting variable $\mu_{0}$ is then adjusted as needed to satisfy 5.44. An interval-splitting method is used to find the appropriate values of $\mu_{0}$, and yields a unique solution in all examples considered. We do not impose 5.45 in the solution process; instead, we impose $z(0)=0$ and compute $z(r)$ 
by forward integrating 5.27. The value of $z\left(r_{0}\right)$ thus obtained is then used to shift the origin of the $z$-axis to fulfill 5.45 a posteriori. This generates a one-parameter shooting problem facilitating global analysis of existence and uniqueness. Consistent with this procedure, we do not assign the height $z_{d}$ of the dipole source above the reference plane. Rather, we assign its height $z_{0}^{d}$ above the center the deformed membrane, which is used as the origin of the $z$-axis in the numerical procedure. This is the operative value of $z_{d}$ in equations 5.38 and 5.39. The actual height of the dipole source above the reference plane is then obtained by computing $z_{d}=z_{d}^{0}-z\left(r_{0}\right)$. In the original statement of the problem $z_{d}$ is considered to be assigned rather than computed. Nevertheless, the actual height $z_{d}$ thus obtained can be correlated with a given value of $z_{d}^{0}$. In this way comparisons can be made at fixed values of actual height.

\subsubsection{Results}

Results for the shooting method are for films with the following properties and dimensions: radius of the film $\left(r_{a}\right)$ of $0.317 \mathrm{~cm}$ film shear modulus $(G)$ of $0.25 \mathrm{MPa}$; Mooney-Rivlin parameter $\delta$ of 0.9 and magnetic susceptibility $(\bar{\chi})$ of 2.5 ; film thickness $(h)$ of $70 \mu \mathrm{m}$; pressure difference $(\Delta p)$ of zero and a variety of dipole strengths $(D)$ and dipole heights $\left(z_{d}\right)$.

The effect of varying the height of a dipole source of fixed intensity $\left(D=0.00269 \mathrm{Am}^{2}\right)$ above the base plane $z=0$ is depicted in Figure 5.2 and 5.3. The hoop stretch at the outer radius is $\Lambda=1$, corresponding to the absence of pre-stretch. The Figure 5.2 shows the shape of the deformed meridian of the membrane at various values of the dipole height $z_{d}$. As expected, the membrane is more severely deformed as the height is decreased (for a monotonically decreasing sequence of height values), due to the ever closer proximity of the source to the membrane material. Figure 5.3 shows how the variation of $z_{d}$ affects the $z$-displacement at the center of the film, denoted by $z_{0}$. This response exhibits a turning point at around $z_{d}=z_{d}^{\mathrm{min}}=.4220 \mathrm{~cm}$ - the minimum source height possible in equilibrium - followed thereafter by increasing values of $z_{d}$ (source farther from the base plane) as $z_{0}$ continues to increase. In a purely mechanical analogue to this problem we would interpret the field exerted on the membrane as a surrogate for a distributed mechanical force. Our results would then indicate that a limit-point instability is encountered as the source height approaches its minimum. It is thus appropriate to conclude that a limit-point instability is encountered in the present problem, and thus that the decreasing branch of the response curve $\left(z_{d}\right.$ increasing), after $z_{d}^{\min }$ is encountered, corresponds to unstable equilibrium. We conjecture that a full dynamic analysis of this problem would predict that, after the equilibrium limit point is reached, the membrane is pulled into the dipole source dynamically. This is confirmed in Chapter 6

Figures 5.4 and 5.5 illustrates the response of the membrane to dipoles of increasing strength held at a fixed distance $\left(z_{d}=0.5 \mathrm{~cm}\right)$ above the base plane. Limit-point behavior is again predicted, corresponding to a maximum dipole strength $\left(D_{\max } \approx 0.00566 \mathrm{Am}^{2}\right)$ for which equilibrium is possible, followed by an increase in pole displacement attending a 


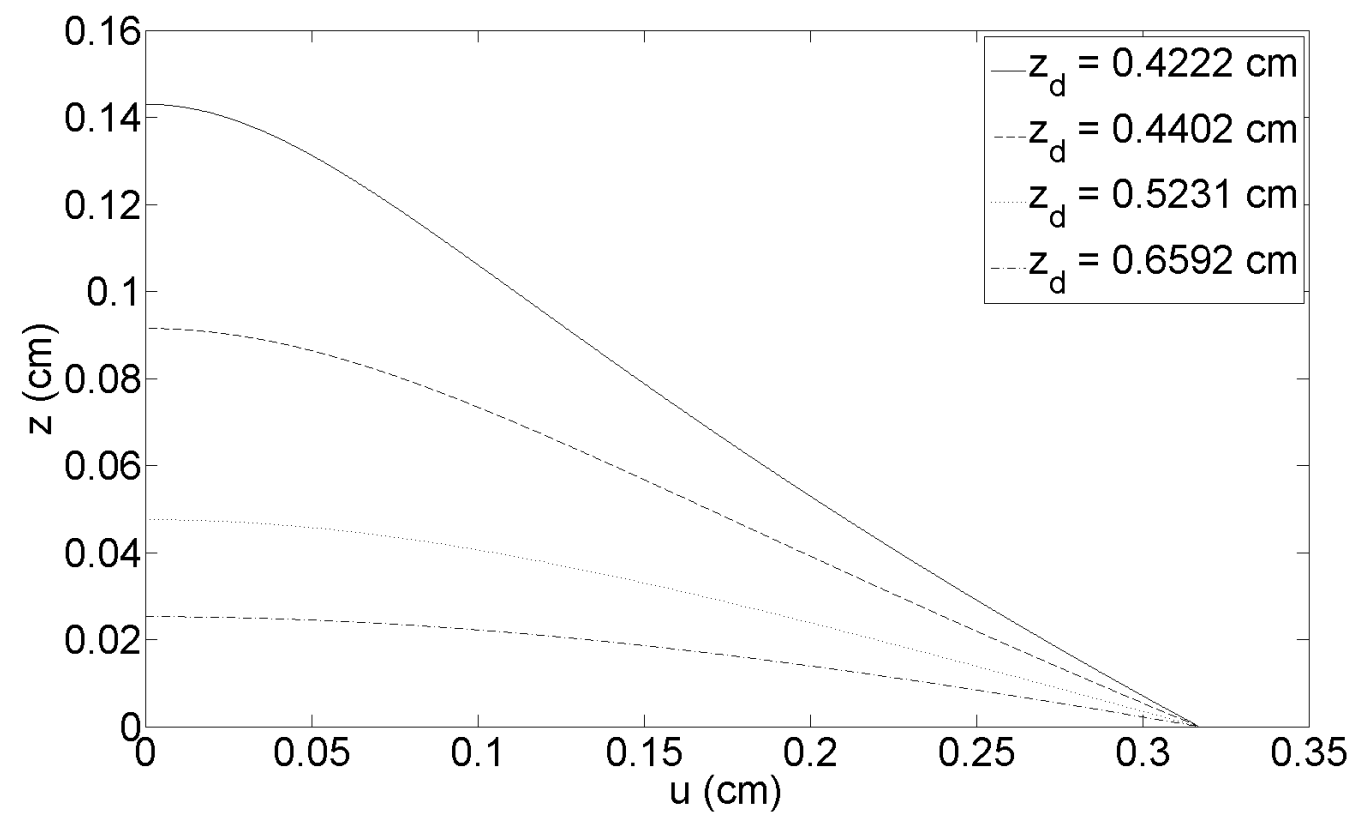

Figure 5.2: Shooting method equilibrium axisymmetric deformation for $D=0.00269 \mathrm{Am}^{2}$, for a range of dipole heights $\left(z_{d}\right)$

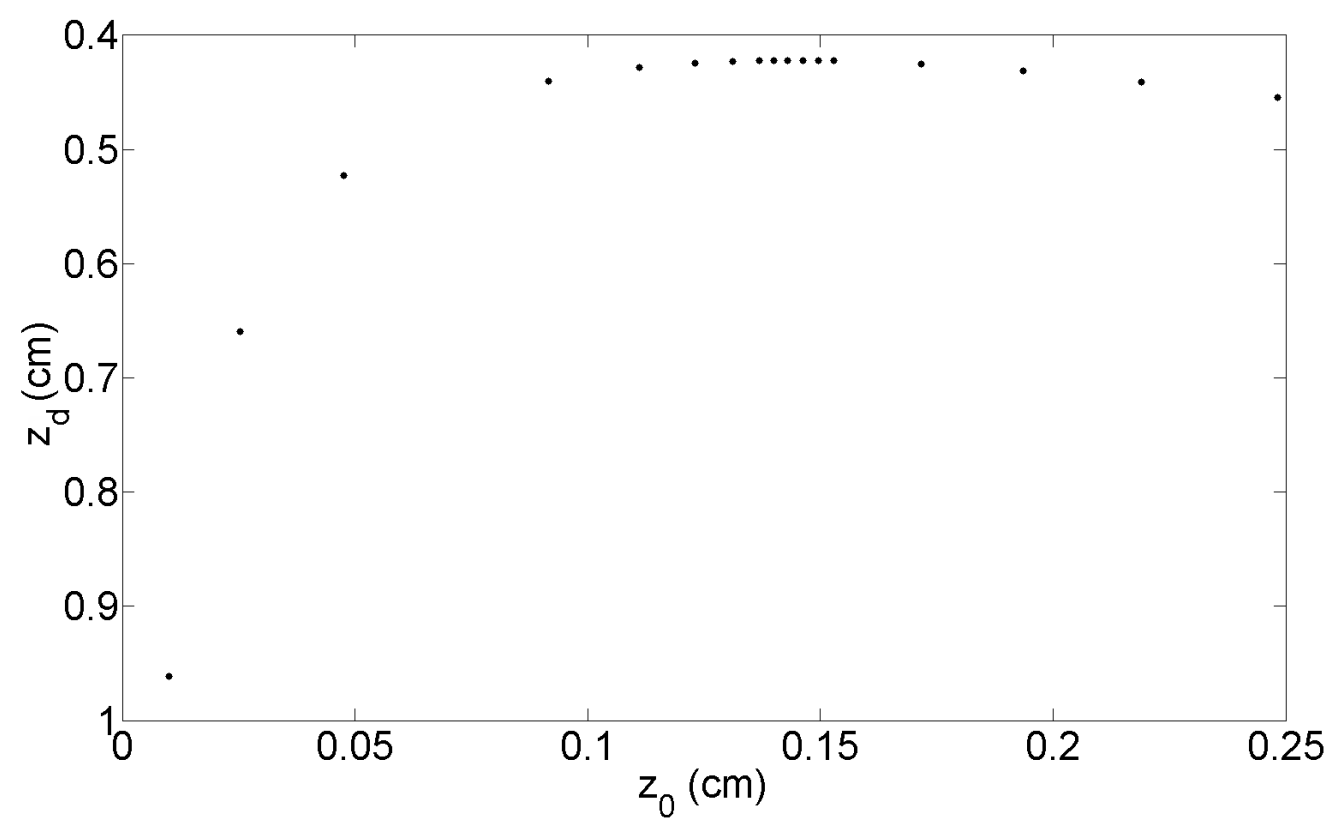

Figure 5.3: Shooting method equilibrium z-displacment at the center of the film for $D=$ $0.00269 \mathrm{Am}^{2}$, for a range of dipole heights $\left(z_{d}\right)$ 
decrease in dipole strength. The response is qualitatively similar to that produced by varying the height of a source of a given intensity.

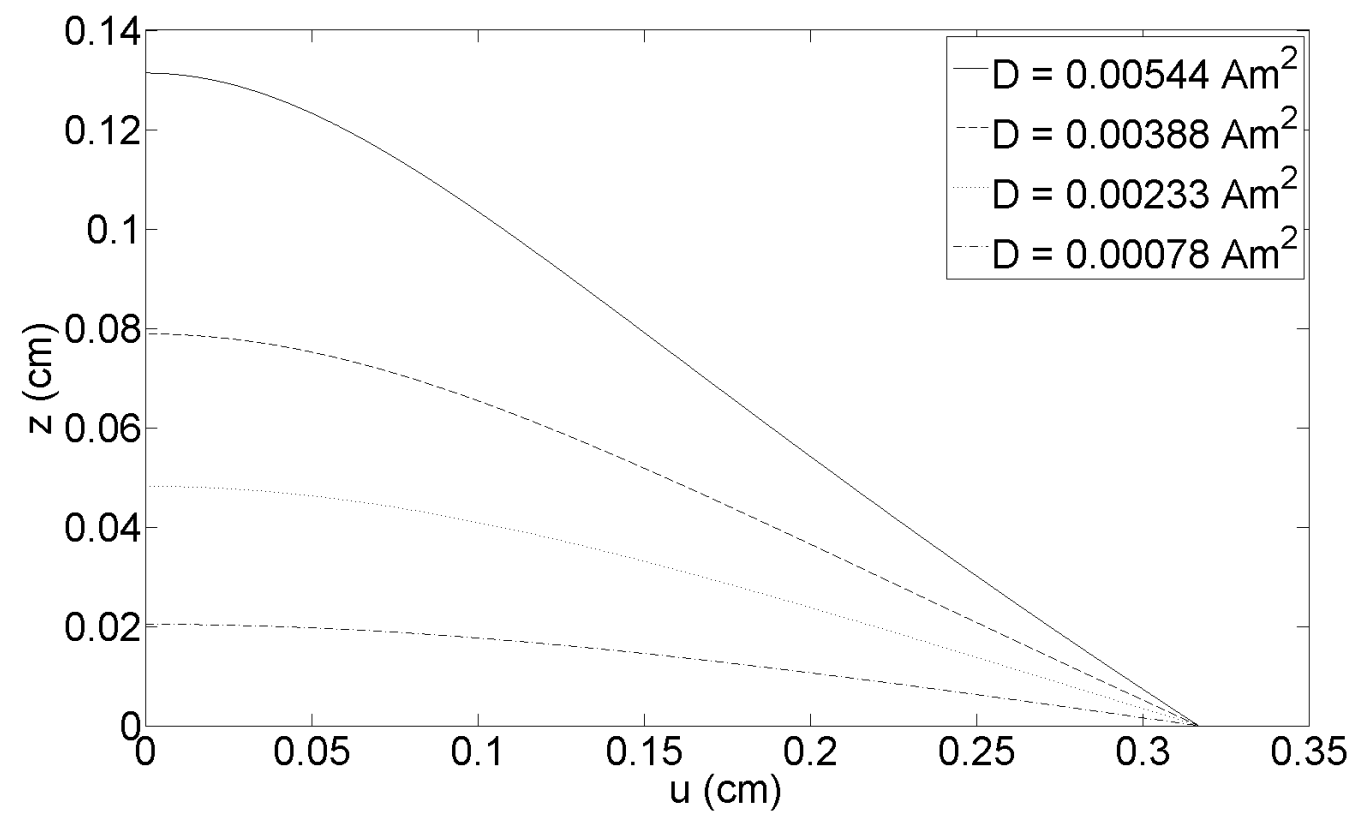

Figure 5.4: Shooting method equilibrium axisymmetric deformation for $z_{d}=0.5 \mathrm{~cm}$, for a range of dipole strengths (D) 


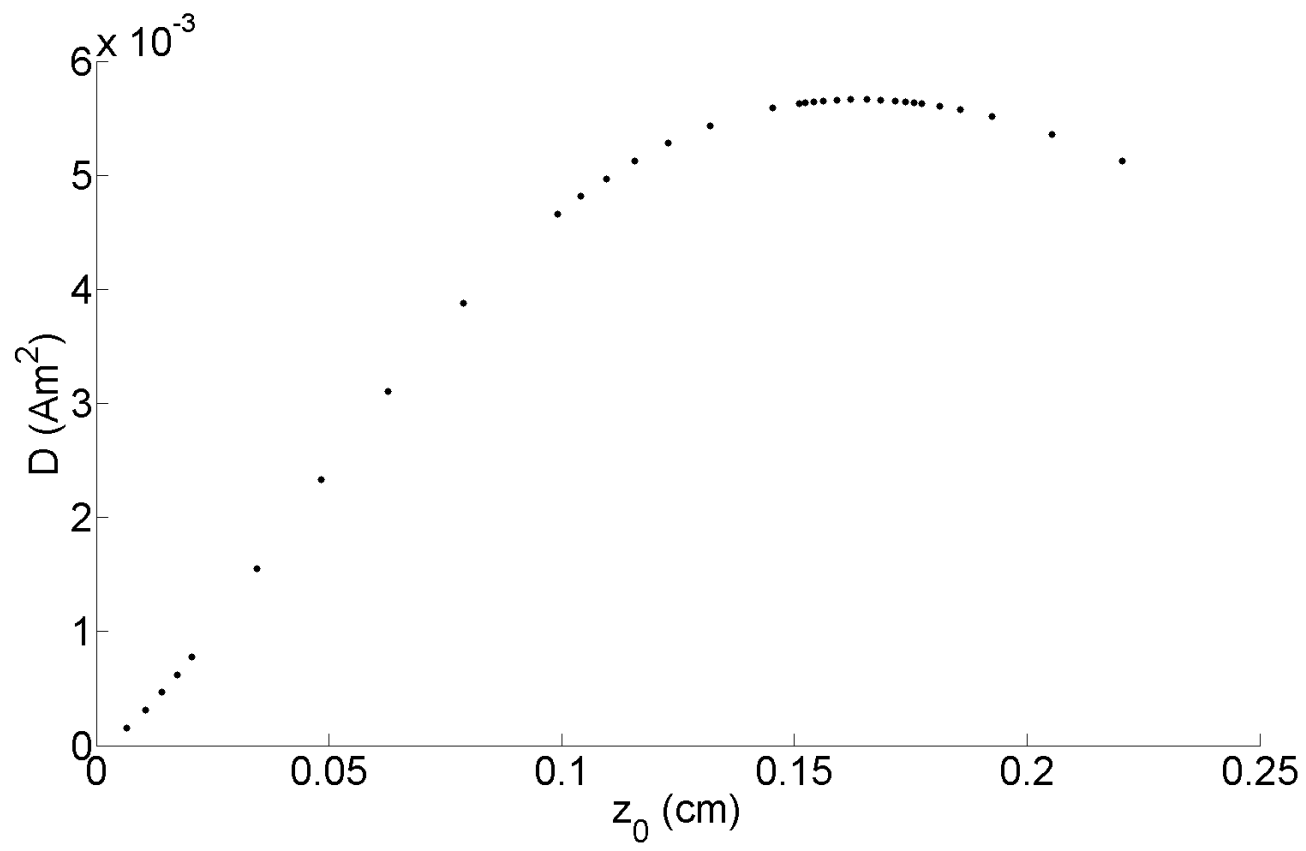

Figure 5.5: Shooting method equilibrium $z$-displacement at the center of the film, for $z_{d}=$ $0.5 \mathrm{~cm}$, for range of dipole strengths $(\mathrm{D})$ 


\subsection{Variational Method}

The variational method is used to show the existence of the limit point instability postulated by the shooting method described in Section 5.1. Note that this instability study has previously been published and the key points are summarized here, see [3] for the compete analysis. Assuming the membrane to be incompressible in bulk $\left(\lambda_{1} \lambda_{2} \lambda_{3}=1\right)$, the constitutive relation between the applied magnetic field and magnetization for a weakly magnetized material (5.8), material property relation of equation (5.10) and the Mooney-Rivlin model for the conventional strain-energy (5.12) the potential energy 3.73 reduces to:

$$
\begin{gathered}
E=\frac{h G}{2} \int_{\Omega}\left[\delta\left(\lambda_{1}^{2}+\lambda_{2}^{2}+\lambda_{1}^{-2} \lambda_{2}^{-2}-3\right)+(1-\delta)\left(\lambda_{1}^{-2}+\lambda_{2}^{-2}+\lambda_{1}^{2} \lambda_{2}^{2}-3\right)\right] d a \\
-\frac{h \mu_{0} \bar{\chi}}{2} \int_{\Omega} \mathbf{h}_{a} \cdot \mathbf{h}_{a} d a-h \int^{V} p^{-}(v) d v-p^{+}
\end{gathered}
$$

where the dimensional thickness $(h)$ is used in place of the dimensionless thickness $(\epsilon)$. The first term is the elastic strain-energy (denoted $E_{\lambda}$ ), the second term is the magnetic energy $\left(E_{h}\right)$ and the third term is the pressure energy $\left(E_{P}\right)$.

We again consider axisymmetric deformations, where the reference mid-surface position is $\mathbf{u}=r \mathbf{e}_{r}(\theta)$ and the current mid-surface position be represented by equation 5.20. To simplify the analysis while emphasizing the dominant features of the response, we make the assumption that there is no deformation along the radial coordinate $(u(r)=r)$. This assumption is made since the deformation in the radial direction is small compared to the axial deformation according to the analysis of the shooting method Section 5.1. Given this assumption the elastic strain-energy in (5.47), in terms of the dimensionless variables $\zeta=z / r_{a}$ and $s=r / r_{a}$, reduces to

$$
E_{\lambda}=\pi a^{2} h G \int_{0}^{1}\left[\left(\zeta^{\prime}\right)^{2}+\frac{1}{1+\left(\zeta^{\prime}\right)^{2}}-1\right] s d s
$$

where $\zeta^{\prime}=d \zeta / d r$

We consider an applied magnetic field generated by the dipole source 2.35 with a dipole position $\mathbf{y}_{d}=z_{d} \mathbf{k}$, invoking the assumption of vanishing radial deformation, and using the dimensionless variables defined previously, the magnetic energy in equation 5.47 simplifies to

$$
E_{h}=-\frac{h \pi \mu_{0} \bar{\chi} D^{2}}{a^{4}} \int_{0}^{1}\left(\frac{s^{2}+4\left[\zeta(s)-\zeta_{d}\right]^{2}}{\left\{s^{2}+\left[\zeta(s)-\zeta_{d}\right]^{2}\right\}^{4}}\right) s d s
$$

where $\zeta_{d}=z_{d} / r_{a}$.

We assume that the pressure transmitted to the membrane is supplied by large reservoirs above and below the film. Thus, deformations of the membrane result in a negligible change in pressure with a volume change. Accordingly, the pressure energy is

$$
E_{P}=-P_{0} \Delta V,
$$


where $P_{0}=p^{+}-p^{-}$is the assigned pressure difference across the membrane and $\Delta V$ is the change in volume. Here, only the change of volume, rather than the volume itself, has physical meaning, and we have taken this fact into account. Assuming axisymmetric deformations and neglecting the radial displacement as above, we obtain

$$
E_{P}=-2 \pi a^{3} P_{0} \int_{0}^{1}[\zeta(s) s] d s .
$$

An understanding of the qualitative features of the response may be gained most easily from a single-degree-of-freedom model of the system. In contrast, the shooting method described in Section 5.1 is concerned with the differential equations of equilibrium, i.e. with an infinite-dimensional model. The latter does not lend itself easily to the analysis of stability. Here we assume the deformed surface to be a one-parameter family of paraboloids defined by

$$
z=z_{0}\left[1-\left(\frac{r}{r_{a}}\right)^{2}\right] \quad \text { or } \quad \zeta=\zeta_{0}\left[1-s^{2}\right]
$$

where $z_{0}$ is the (unknown) axial deformation at the center of the membrane and $\zeta_{0}=z_{0} / a$, see Figure 5.6.

To assess the accuracy of the simple single-degree-of-freedom model, we consider a twoparameter representation, again using the axial deformation at the center $\left(z_{0}\right)$ as a parameter. In principle, this model should produce more accurate results than the one-parameter model. The assumed mode of deformation is

$$
z=z_{0}\left[1-\left(\frac{r}{r_{a}}\right)^{2}\right]+c\left[\left(\frac{r}{r_{a}}\right)^{3}-\left(\frac{r}{r_{a}}\right)^{2}\right] \quad \text { or } \quad \zeta=\zeta_{0}\left[1-s^{2}\right]+b\left[s^{3}-s^{2}\right]
$$

where $b=c / r_{a}$, also unknown. The second term in equation 5.53 makes no contribution to the displacement of the membrane at the center or the edge and incorporates the symmetry condition of zero slope at the center, see Figure 5.6.

The variational approach used depends on the number of parameters that are varied. We discuss the one- and two- parameter models in turn. In the one-parameter case the total energy has been determined as a function of $z_{0}$. The first variation of the energy is taken with respect to $z_{0}$. If the first variation vanishes, then the deformation is in equilibrium at the associated value of the parameter. Thus, we require

$$
\frac{\partial E}{\partial \zeta_{0}}=\frac{\partial E_{\lambda}}{\partial \zeta_{0}}+\frac{\partial E_{h}}{\partial \zeta_{0}}+\frac{\partial E_{P}}{\partial \zeta_{0}}=0 .
$$

The value of $\zeta_{0}$ that satisfies the above equation corresponds to an equilibrium state; this relative deflection at equilibrium is denoted by $\zeta_{e}$. To assess the stability of the equilibrium state thus obtained, we compute the second variation:

$$
\frac{\partial^{2} E}{\partial \zeta_{0}^{2}}=\frac{\partial^{2} E_{\lambda}}{\partial \zeta_{0}^{2}}+\frac{\partial^{2} E_{h}}{\partial \zeta_{0}^{2}}+\frac{\partial^{2} E_{P}}{\partial \zeta_{0}^{2}} .
$$




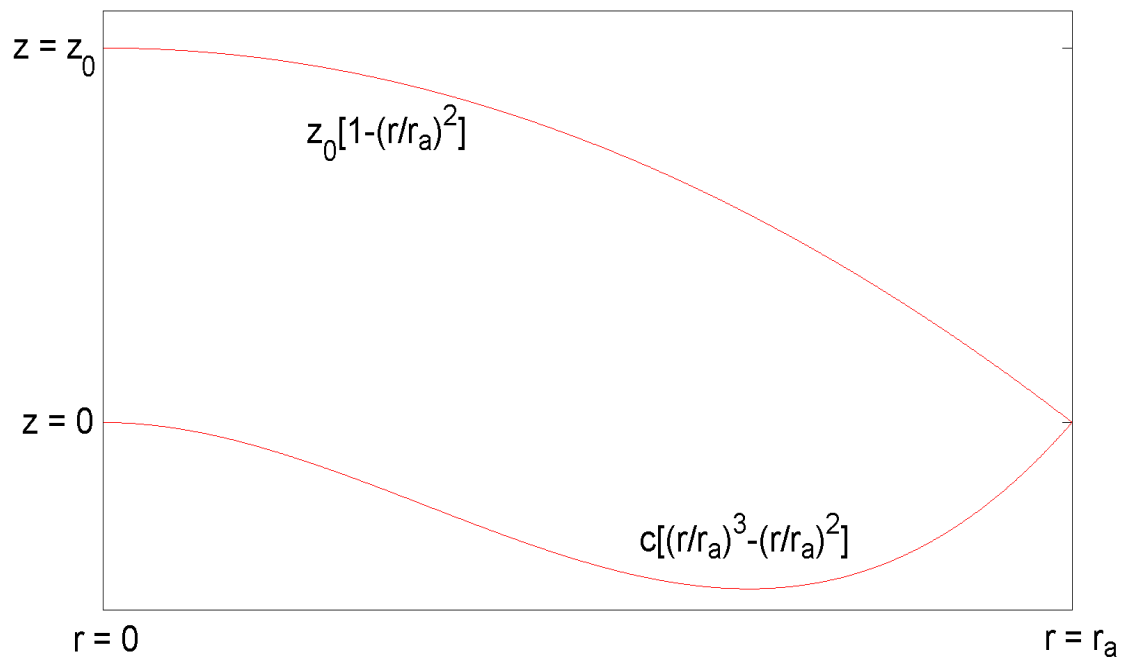

Figure 5.6: Degrees of freedom used in the variational method.

evaluated at $\zeta_{0}=\zeta_{e}$. If the second variation is positive, then the equilibrium deformation is judged stable. If negative, the equilibrium is unstable, and it is neutrally stable if the second variation vanishes.

In the two-parameter model, the first variation of the energy is taken with respect to $\zeta_{0}$ and $b$. Again, the deformation is equilibrated when the first variation vanishes. Such deformations satisfy

$$
\frac{\partial E}{\partial \zeta_{0}}=\frac{\partial E_{\lambda}}{\partial \zeta_{0}}+\frac{\partial E_{h}}{\partial \zeta_{0}}+\frac{\partial E_{P}}{\partial \zeta_{0}}=0 \quad \text { and } \quad \frac{\partial E}{\partial b}=\frac{\partial E_{\lambda}}{\partial b}+\frac{\partial E_{h}}{\partial b}+\frac{\partial E_{P}}{\partial b}=0 .
$$

We denote the associated parameter values by $\zeta_{e}$ and $b_{e}$. The considered equilibrium state is stable if the matrix:

$$
\left[\begin{array}{ll}
\frac{\partial^{2} E}{\partial \zeta_{0}^{2}} & \frac{\partial^{2} E}{\partial \zeta_{0} \partial b} \\
\frac{\partial^{2} E}{\partial b \zeta_{0}} & \frac{\partial^{2} E}{\partial b^{2}}
\end{array}\right],
$$

is positive definite at $\zeta_{0}=\zeta_{e}$ and $b=b_{e}$. If this matrix is negative definite or indefinite, i.e. if any eigenvalue is negative, then the deformation is unstable. If one eigenvalue vanishes and the other is positive or vanishes, the deformation is (locally) neutrally stable. If both eigenvalues are positive, the deformation is (locally) stable.

Equations 5.54 and 5.55 for the one-parameter model, and equations 5.56 and 5.57 for the two-parameter model, despite their simplicity relative to the exact theory, are too complicated to be solved analytically. Accordingly, we adopt a numerical solution to determine 
the elevation of the dipole above the undeformed membrane at which the equilibrium state first becomes unstable; this height is denoted by $\zeta_{i}$. For the one- parameter model, plots of $E, \partial E / \partial \zeta_{0}$ and $\partial^{2} E / \partial \zeta_{0}^{2}$ verses $\zeta_{0}$ were generated. For the two-parameter model, plots of $E, \partial E / \partial \zeta_{0}$ and $\partial E / \partial b$ over the $\zeta_{0}-b$ plane, and both eigenvalues of the second-variation matrix, were obtained.

In both models it was observed that equilibrium deformations exist for a fixed $\zeta_{d}$ corresponding to a local minimum of the energy. A simple numerical scheme was used to determine if a local minimum exists for a given value of $\zeta_{d}$. In this scheme we first calculate the energy over a range of $\zeta_{0}$ values for the one-parameter deformation and over a range of $\zeta_{0}$ and $b$ for the two-parameter deformation. Then, we determine if there is a local minimum. In the negative case, we increase the assumed value of $\zeta_{d}$ by a small amount, $\Delta \zeta_{d}$, until a local minimum is obtained. Then, we decrease $\Delta \zeta_{d}$ by a factor of 10 and use the last unstable value of $\zeta_{d}$, adding $\Delta \zeta_{d}$ to obtain a new guess of $\zeta_{d}$. This process is repeated until the desired number of significant figures is attained and the last stable value of $\zeta_{d}$ is recorded as $\zeta_{i}$. For the one- parameter deformation, the values of $\partial E / \partial \zeta_{0}$ and $\partial^{2} E / \partial \zeta_{0}^{2}$ at $\zeta_{i}$ are checked. For the two-parameter deformation $\partial E / \partial \zeta_{0}, \partial E / \partial b$ and both eigenvalues of the second-variation matrix at $\zeta_{i}$ are checked.

The calculations were performed using the following parameter values: $r_{a}=0.317 \mathrm{~cm}$, $h=70 \mu \mathrm{m}, G=0.25 \mathrm{MPa}, \rho=1722 \mathrm{~kg} / \mathrm{m}^{-3}, \bar{\chi}=2.5, \mu_{0}=4 \pi \times 10^{-7} \mathrm{~N} / \mathrm{A}^{2}, \delta=0.9$ and $P_{0}=0$, and for different values of the magnetic dipole strength, $D$. The results for the one-parameter and two-parameter models are depicted in table 5.1 and compared with the peak-response values inferred from the numerical solution of the differential equations of the exact theory for the shooting method described in Section 5.1.

We observe that the inferred values $\zeta_{i}$ using the shooting method in Section 5.1 and the values calculated from the present models are in quantitative agreement, with the twoparameter deformation producing more accurate results, as expected. The per cent errors of the present models relative to the exact solution are shown in table 5.1.

\begin{tabular}{|l|l|l|l|l|l|}
\hline$D\left(A m^{2}\right)$ & $\zeta_{i}$ Shooting & $\zeta_{i}$ 1-par. & 1-par \% error & $\zeta_{i}$ 2-par & 2-par \% error \\
\hline \hline $2.69 \times 10^{-4}$ & 0.7868 & 0.6937 & 11.833 & 0.7616 & 1.494 \\
$2.69 \times 10^{-3}$ & 1.3310 & 1.2334 & 7.332 & 1.3111 & 3.203 \\
$2.69 \times 10^{-2}$ & 2.2540 & 2.1679 & 3.821 & 2.2427 & 0.503 \\
$2.69 \times 10^{-1}$ & 3.8664 & 3.7960 & 1.820 & 3.8628 & 0.094 \\
$2.69 \times 10^{0}$ & 6.7533 & 6.6841 & 1.025 & 6.7477 & 0.082 \\
\hline
\end{tabular}

Table 5.1: $\zeta_{i}$ values and per cent error for the one- and two-parameter representations.

Another interesting observation, confirming the conjecture offered in Section 5.1, is that the instability is of the limit-point type. This becomes evident when $\zeta_{d}$ approaches the 
instability height. In the single-degree-of-freedom model, the equilibrium values of $\zeta_{0}$ corresponding to a stable and to an unstable solution are seen to coalesce. When the instability height is attained, the two solutions coincide. No equilibrium state, and hence no stable equilibrium state, exists if the source height decreases below this value. This behavior is depicted in Figure 5.7 for a particular value of dipole strength.

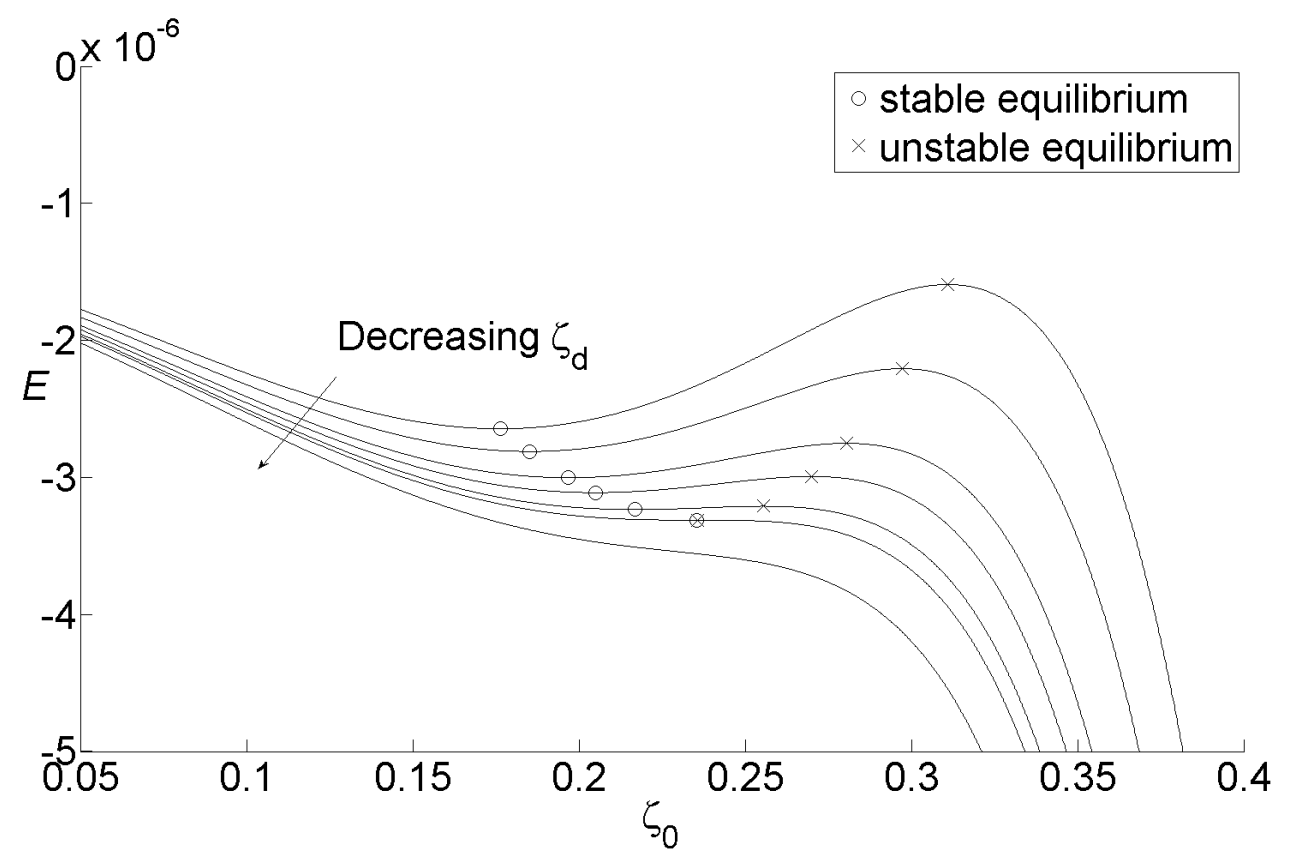

Figure 5.7: Energy (joules) versus the pole deformation $\zeta_{0}$ for various dipole heights above the reference plane.

The limit-point nature of instability is not so readily apparent in the two-parameter model. In order to discern it, we plot (in Figures 5.8-5.10) the three-dimensional energy versus $\zeta_{0}$ and $\mathrm{b}$ together with a contour plot of the energy at three values of $\zeta_{d}$. Stable equilibria are marked with a dot. The first is at a stable value of $\zeta_{d}\left(\zeta_{d}>\zeta_{i}\right)$, the second at the incipient instability height $\zeta_{d}\left(\zeta_{d}=\zeta_{i}\right)$ and the third at an unstable value of $\zeta_{d}\left(\zeta_{d}<\zeta_{i}\right)$. In Figures 5.8-5.10 it may be seen that, as $\zeta_{d}$ decreases, the size of the cup where the local minimum is located decreases in size, until $\zeta_{d}=\zeta_{i}$, when the cup is no longer present and beyond that point no local minimum reappears.

The advantage of the energy criterion of elastic stability, extended herein to the magnetoelastic setting, is that the stability or instability of the deformation may be assessed based on the considered equilibrium state alone. It is particularly amenable to use a finitedimensional model of the system, and this fact is exploited here to give an approximate analysis of stability. As expected, the quantitative agreement between our predictions and the limit points predicted using the exact differential equations of weakly magnetized ma- 


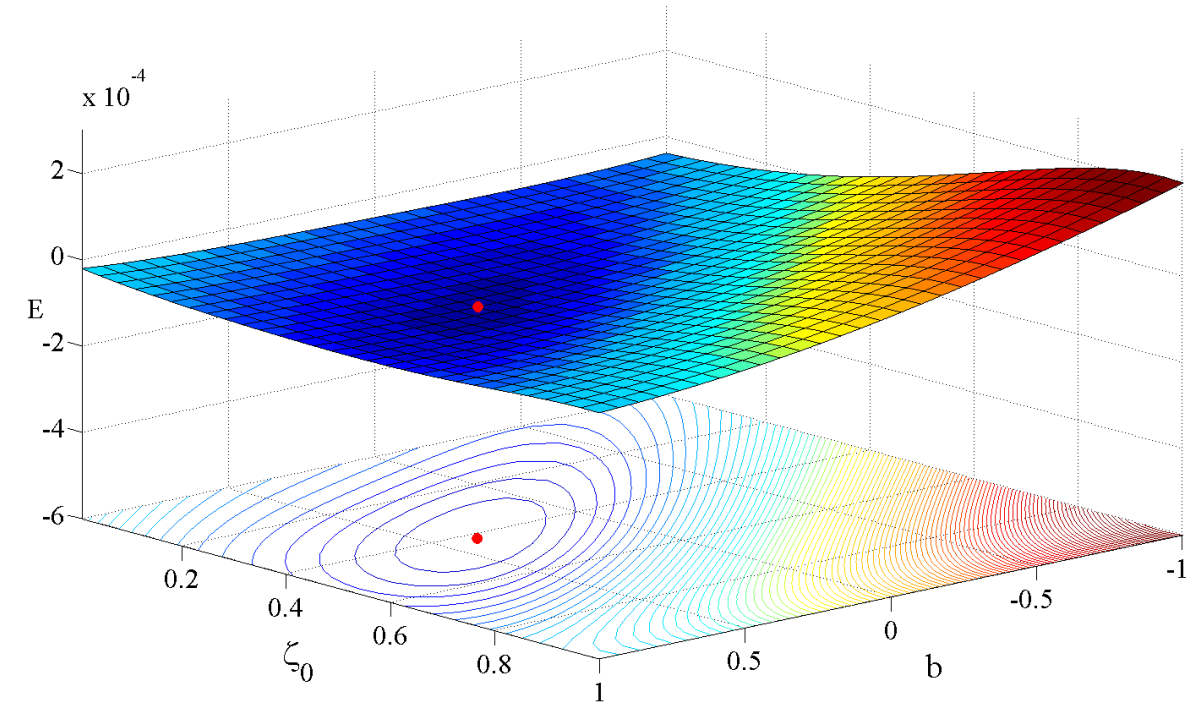

Figure 5.8: Energy (joules) versus $\zeta_{0}$ versus $b$ for $\zeta_{d}=2.33$, and $D=0.02688 A m^{2}$. Stable equilibrium marked with a dot, $b^{\text {min }}=0.37931$ and $\zeta_{0}^{\min }=0.41966$.

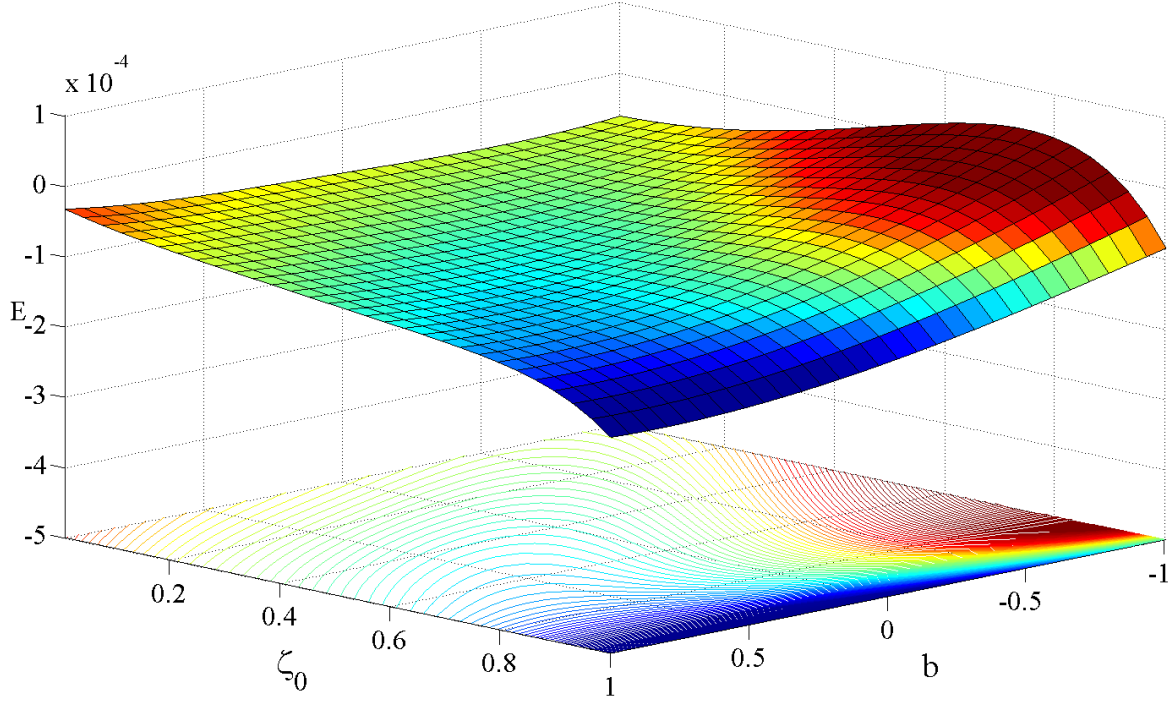

Figure 5.9: Energy (joules) versus $\zeta_{0}$ versus $b$ for $\zeta_{d}=2.2$. No equilibria present. 


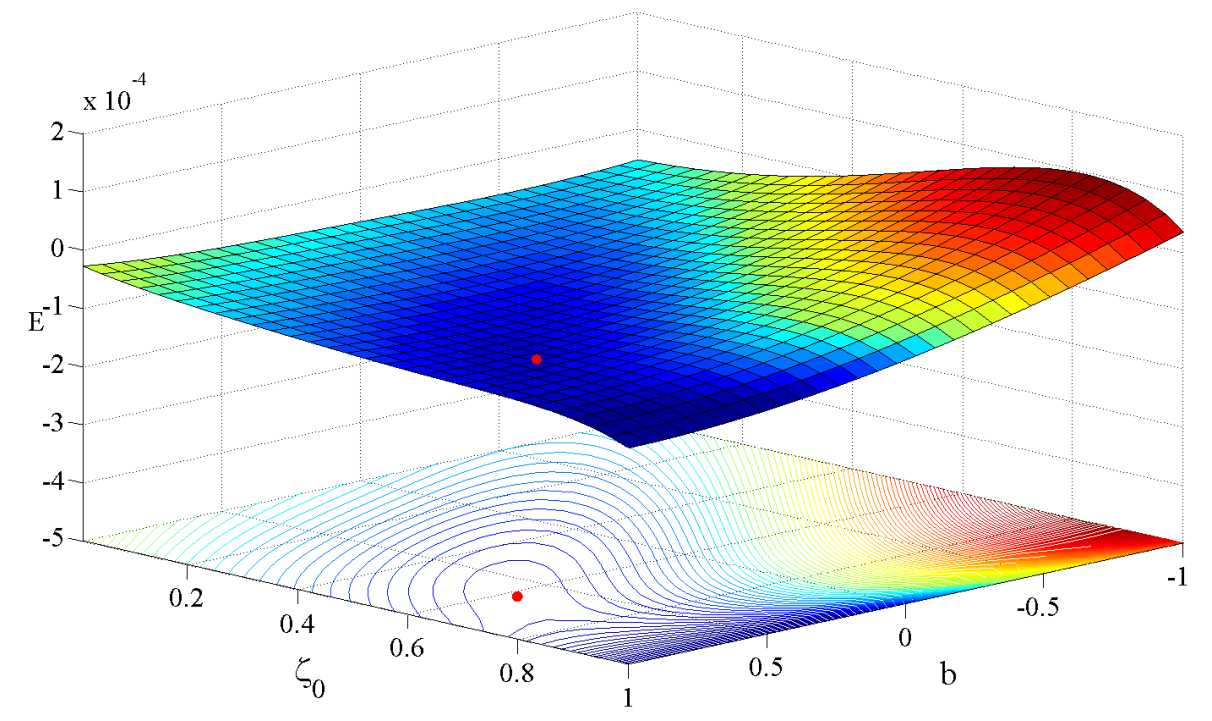

Figure 5.10: Energy (joules) versus $\zeta_{0}$ versus $b$ for $\zeta_{d}=\zeta_{i}=2.2427$. Incipient unstable equilibrium marked with a dot, $b^{\text {min }}=0.65517$ and $\zeta_{0}^{\text {min }}=0.62448$.

terial solved with the shooting method in Section 5.1 is seen to improve in passing from the one- to the two-degree-of-freedom model. Given the high accuracy achieved with these simple representations, it is believed that a more refined finite-element representation is redundant for the problem considered. The results of the analysis confirm the earlier conjecture in Section 5.1 that a limit-point instability is encountered when the dipole source and the membrane are in close proximity.

\subsection{Finite Element Method}

This section has previously been published in [4], also note that we performed a more detailed analysis of the Finite Element Method (FEM) presented here in [5]. The FEM is based on a coupling of the full 3D equations of dynamic elasticity coupled with low-frequency (eddy current approximation) electromagnetics. The elastic equations are solved using the standard Galerkin method with linear nodal basis functions and implicit Hilber-HughesTaylor integration [16]. This integration method applies a controlled amount of damping to high-frequency oscillations, which aids convergence to steady-state deformation. The magnetic equations are solved using an $\mathrm{H}$ (curl)-conforming Galerkin method with implicit time integration [30]. The elastic equations and the magnetic equations are coupled using an operator-splitting approach. The software is based on an existing magnetohydrodynamics code [31], with the addition of the hyperelastic Mooney-Rivlin model for the stress-strain relationship. The computational mesh includes the film and a region of vacuum surrounding 
the film. In the film, the mesh moves with the material (pure Lagrangian) but in the vacuum around the film, the mesh is allowed to relax, Arbitrary Eularian Legrangian (ALE). This ALE relaxation prevents the mesh from becoming highly distorted for large film displacements.

A steel ring with a prescribed current is located above the membrane to create a magnetic field similar to a magnetic dipole. The distance between the midplane of the film and the midplane of the ring is $z_{d}$, see Figure 5.1 for a schematic of the problem. To minimize the size of the problem, the axisymmetry of the system is used to mesh only a quarter of a revolution around the axis of symmetry and impose a symmetry boundary condition on the $x=0$ and $y=0$ planes. We also take advantage of the fact that the magnetic field produced above and below the center of the current carrying ring is symmetric, at the $z=2 z_{d}$ plane. Thus, we only mesh the region below the center of the current carrying ring. See Figures 5.11 and 5.12 for the FEM layout and material regions.

The magnetic boundary conditions are as follows: on the ends of the current carrying ring at $y=0$, the normal component of the current density is set to $J$, and on the other end of the wire at $x=0$, the normal component of the current density is set to $-J$; on all other free surfaces, the normal component of the current density is set to zero; on the top of the mesh (symmetry plane at $z=2 z_{d}$ ), the tangential component of the magnetic field is set to zero; on all other faces, the tangential electric field is set to zero, thus insuring that the normal component of the magnetic field is zero.

On all free surfaces that are not symmetry planes, the displacement is constrained. The displacement on the edge of the film that is not on a symmetry plane is also constrained. The entire edge of the film is not constrained since this would impose zero slope as well as the desired zero displacement. To get a better comparison to the shooting method discussed in Section 5.1, only the nodes on the midplane are constrained. This constraint allows for rotation at the edge of the film while maintaining zero displacement of the midplane at the edge.

The elastic response of the film is modeled using the Mooney-Rivlin strain-energy function see equation 5.12. Since the current carrying loop does not produce an exact magnetic dipole field, an equivalent dipole strength $\left(D_{e q}\right)$ for comparison of the two methods needs to be calculated. The ALE finite-element code is initially run in static mode (no motion) to determine the steady-state magnetic field. An equivalent dipole strength is calculated for each element between $0 \leq r \leq r_{a}$ and $\left(z_{d}+h / 2\right) \leq z \leq 7 z_{d} / 5$. The equivalent dipole strength is calculated based on the $z$-component of the magnetic field $\left(h_{z F E}\right)$, since it is much larger than the radial component. The average of these values is used to calculate the equivalent dipole strength. The equivalent dipole strength is determined by taking the dot product of 2.35 with $\mathbf{k}$, and setting it equal to the axial component of the of the magnetic field produced in the finite-element code $\left(h_{z F E}\right)$ resulting in:

$$
h_{z F E}=D_{e q} \frac{2\left(z_{c}-z_{d}\right)^{2}-r_{c}^{2}}{\ell^{5}}
$$




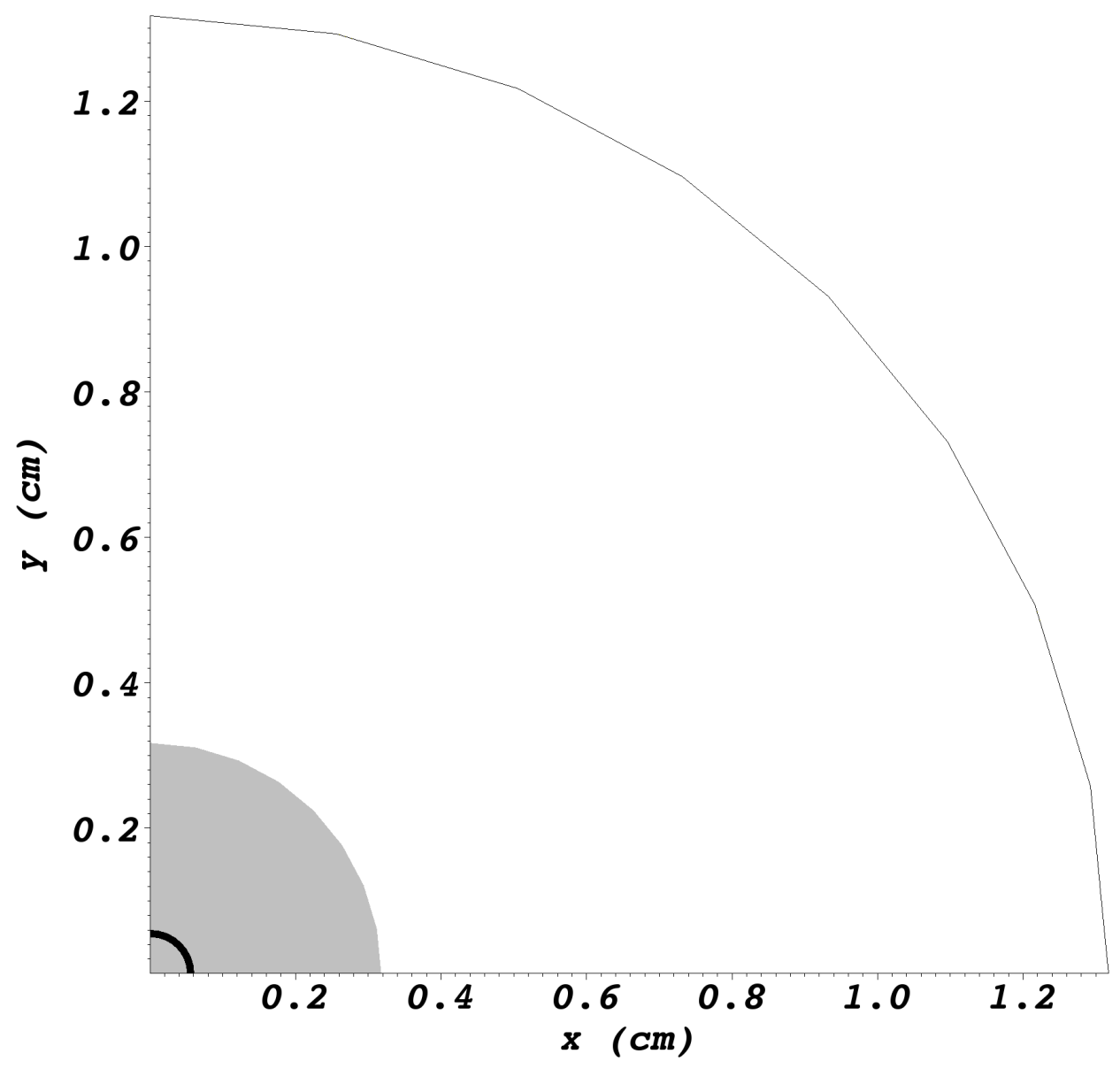

Figure 5.11: Top view of FEM material regions (air is white, steel is black, and film is gray). 


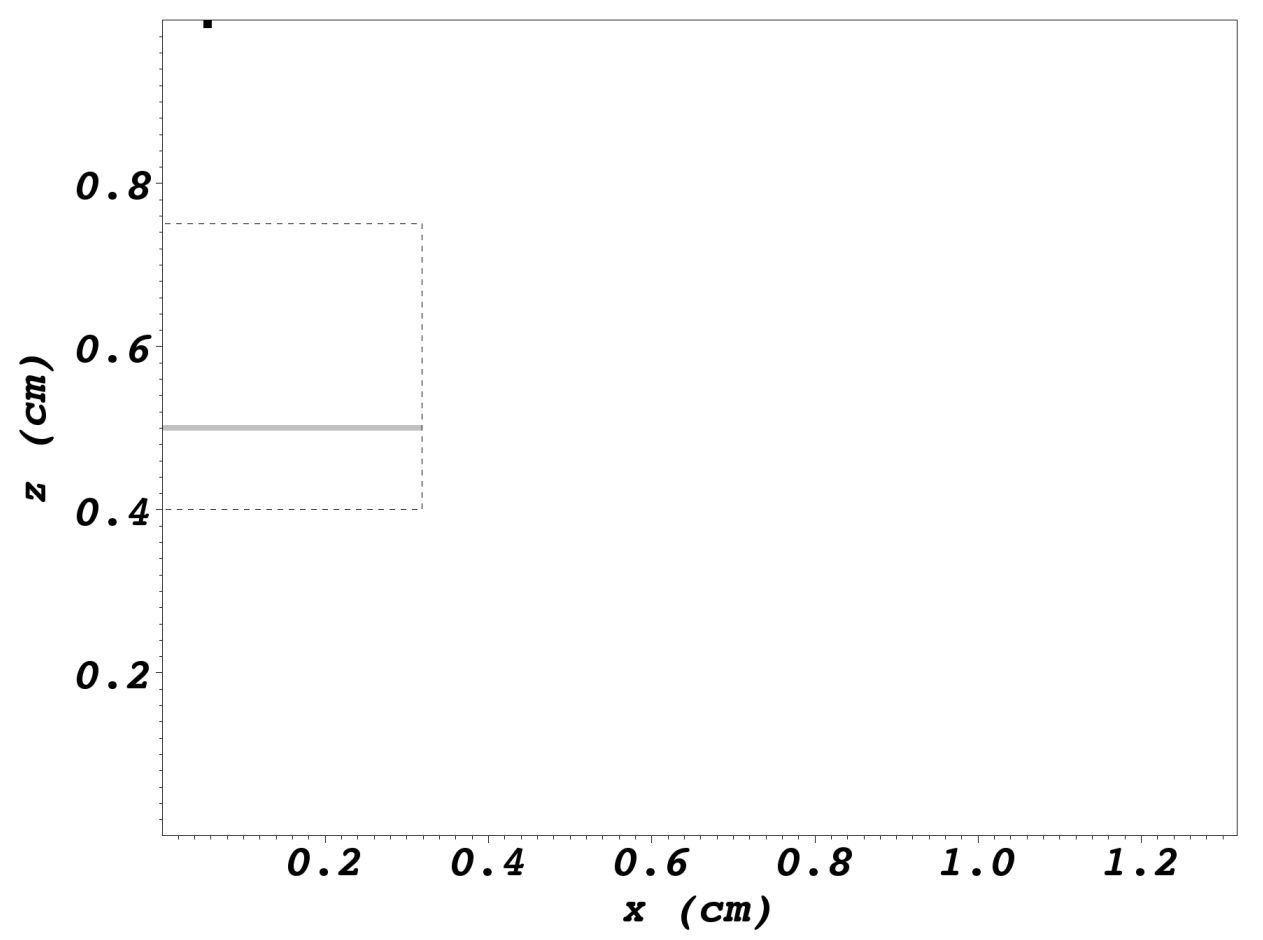

Figure 5.12: Side view of FEM material regions (air is white, steel is black, and film is gray) and the region allowed to advect is inside the dashed line.

and when solved for the equivalent dipole strength

$$
D_{e q}=\frac{h_{z F E} \ell^{5}}{2\left(z_{c}-z_{d}\right)^{2}-r_{c}^{2}}
$$

where $z_{c}$ is the height of the center of the element above the midpane of the film; and $r_{c}$ is the radial position of the center of the element away from the axis of symmetry. It should be noted that the relationship between the equivalent dipole strength and the current density is linear. Thus, once the equivalent dipole strength is calculated for one current density, the equivalent dipole strength for any other current density can easily be calculated.

Once the equivalent magnetic field is determined, the finite-element code is used in the dynamic mode. In order to avoid an undesirable shock to the film, the magnitude of the current is slowly ramped up to the final value. Results for the FEM are for films with the following properties and dimensions: radius of the film $\left(r_{a}\right)$ of $0.317 \mathrm{~cm}$ film shear modulus $(G)$ of $0.25 \mathrm{MPa}$; Mooney-Rivlin parameter $\delta$ of 0.9 ; magnetic susceptibility $(\bar{\chi})$ of 2.5 ; dipole height $z_{d}$ of $0.5 \mathrm{~cm}$; a variety of film thickness and dipole strengths $(D)$.

When the magnetic field of the FEM model is compared to that of a dipole, for elements used to calculate the equivalent dipole strength, the average error in the radial and 
axial direction are $3.3 \%$ and $1.7 \%$, respectively. This is quite good considering the different approximations used.

Figure 5.13 shows the full 3D equilibrium deformation and magnetic field at an equivalent dipole strength of $0.00544 \mathrm{Am}^{2}$ for the region allowed to advect. Figure 5.14 shows slices of the equilibrium position of the film from the FEM at various dipole strengths. If the equivalent magnetic dipole strength of the FEM model is increased above $0.0059 \mathrm{Am}^{2}$, for a film thickness of $70 \mu \mathrm{m}$, the FEM model becomes unstable (dynamic) and no static equilibrium is attained. This is consistent with the instability postulated in Section 5.1 and examined in Section 5.2.

Two comparisons are made between the shooting method and FEM. Figure 5.15 has a comparison of the midplane deformation for a variety of dipole strengths. It is seen that the deformations for the two methods are very similar, validating the both methods. Figure 5.16 has a comparison of the midplane displacement at the center of the film for a variety of dipole strengths. The two models agree well for the stable equilibria. We have also plotted unstable equilibria predicted by the shooting method $\left(z_{0}>0.15 \mathrm{~cm}\right)$; the steady state FEM model does not find these solutions because the membrane goes dynamic.

Comparisons of the midplane displacement at the center of the film were also made for varying film thickness for a variety of small equivalent dipole strengths; see Figure 5.17. It is seen that as the thickness of the film in the FEM decreases, it approaches the membrane model as expected.

The agreement of the results from the FEM and shooting models provides validation for both the membrane model solved by the shooting method and the FEM model. 


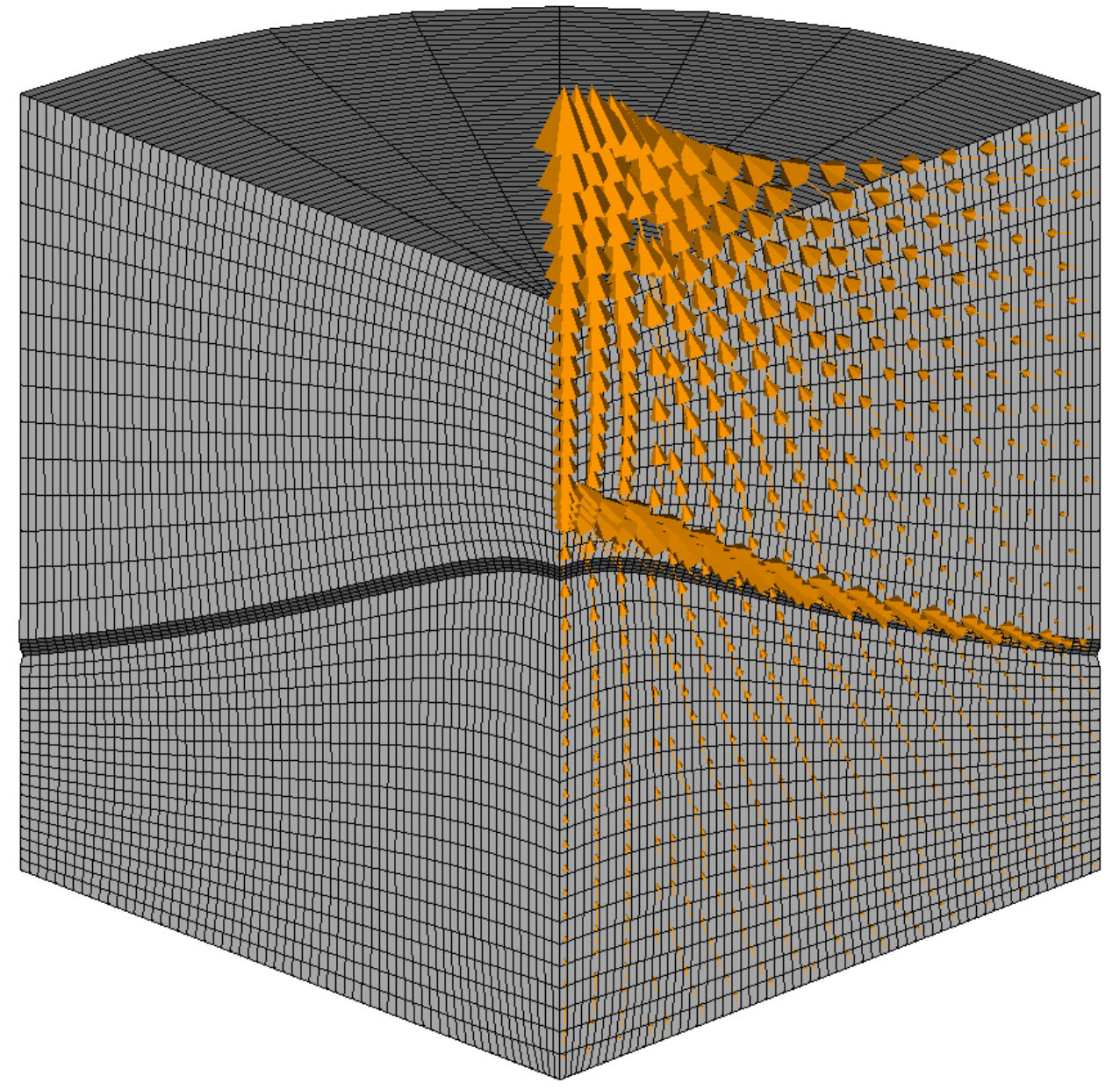

Figure 5.13: Deformed film and magnetic field (orange vectors) for the FEM model at $D_{e q}=0.00544 \mathrm{Am}^{2}$ 


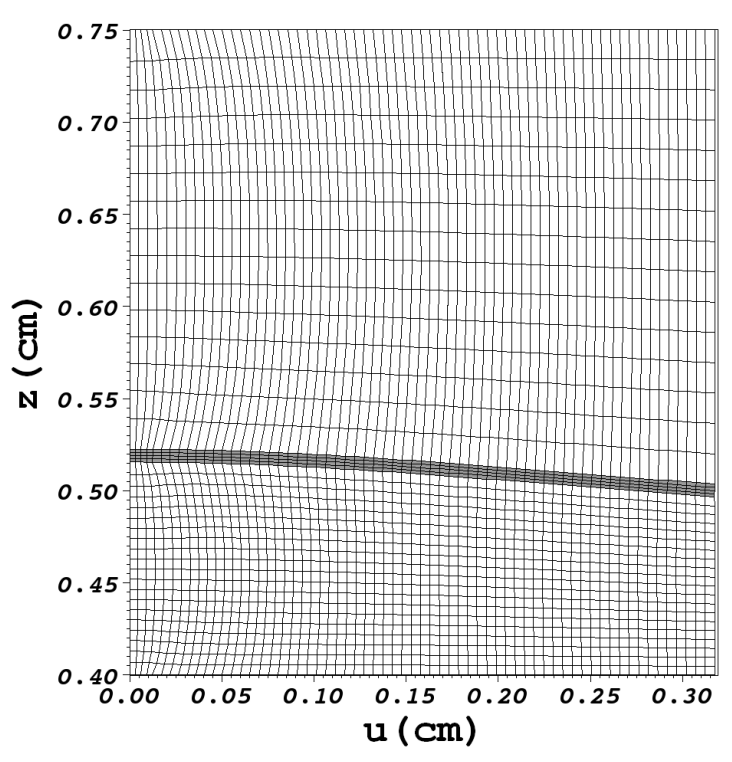

(a) $D_{\text {eq }}=0.00078 \mathrm{Am}^{2}$

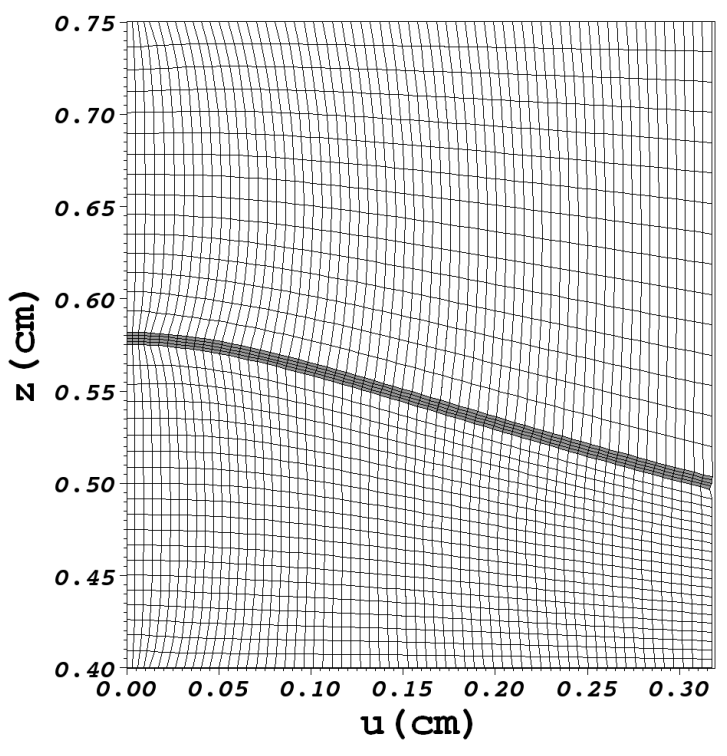

(c) $D_{e q}=0.00388 \mathrm{Am}^{2}$

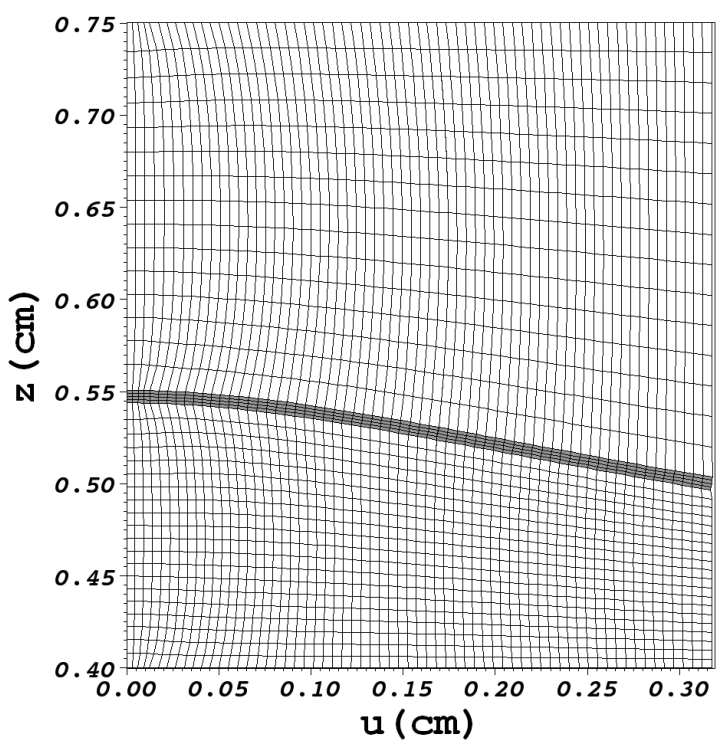

(b) $D_{e q}=0.00233 \mathrm{Am}^{2}$

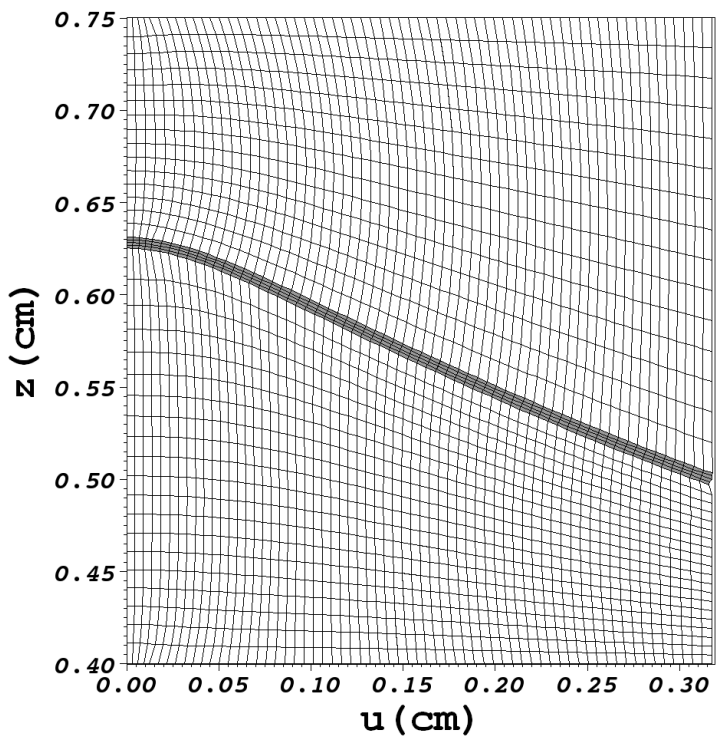

(d) $D_{e q}=0.00544 \mathrm{Am}^{2}$

Figure 5.14: 2D cross section of displacement for the FEM model. 


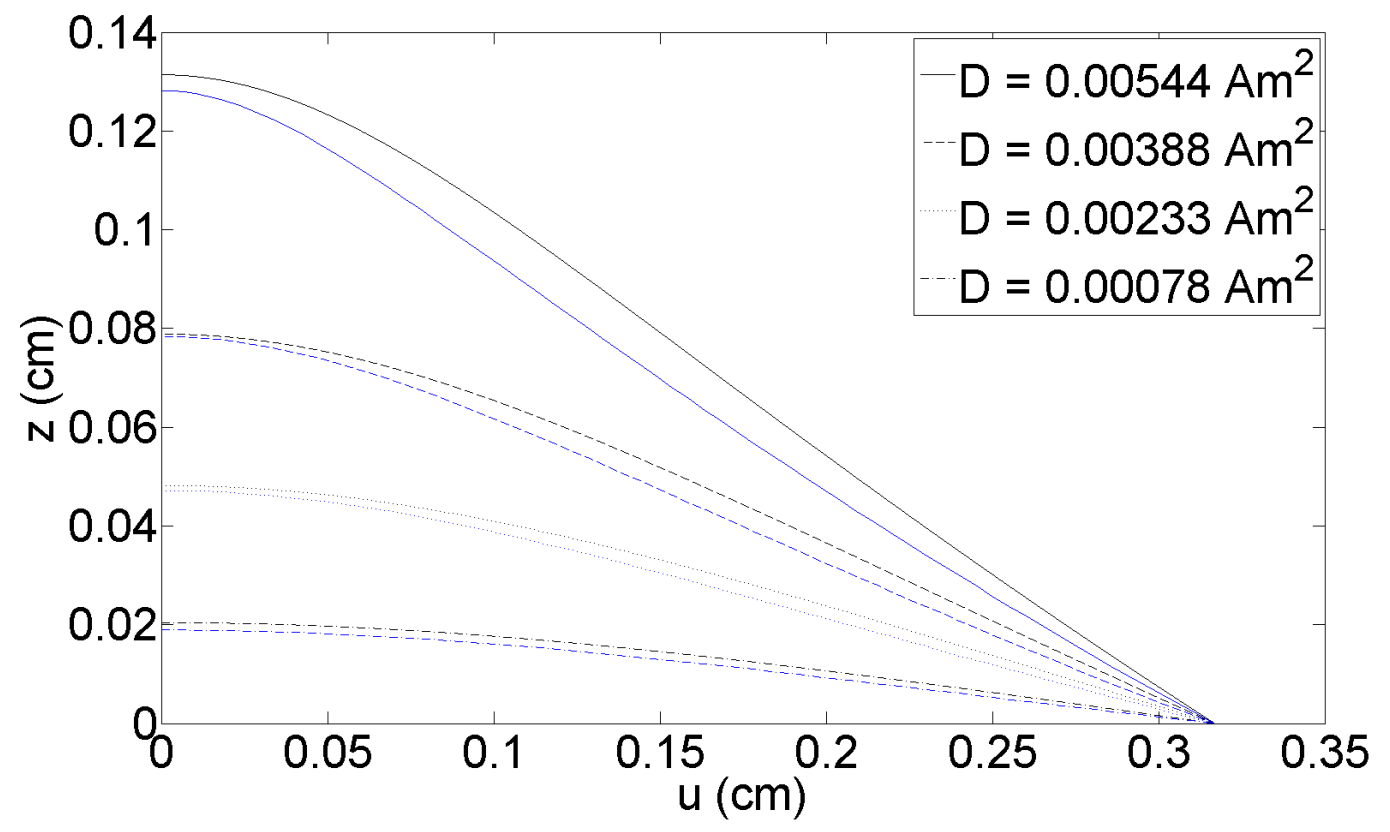

Figure 5.15: Comparison of displacement, shooting method vs. FEM. The black curves are the shooting method results and the blue curves are the FEM results for a film thickness of $h=70 \mu m$. 


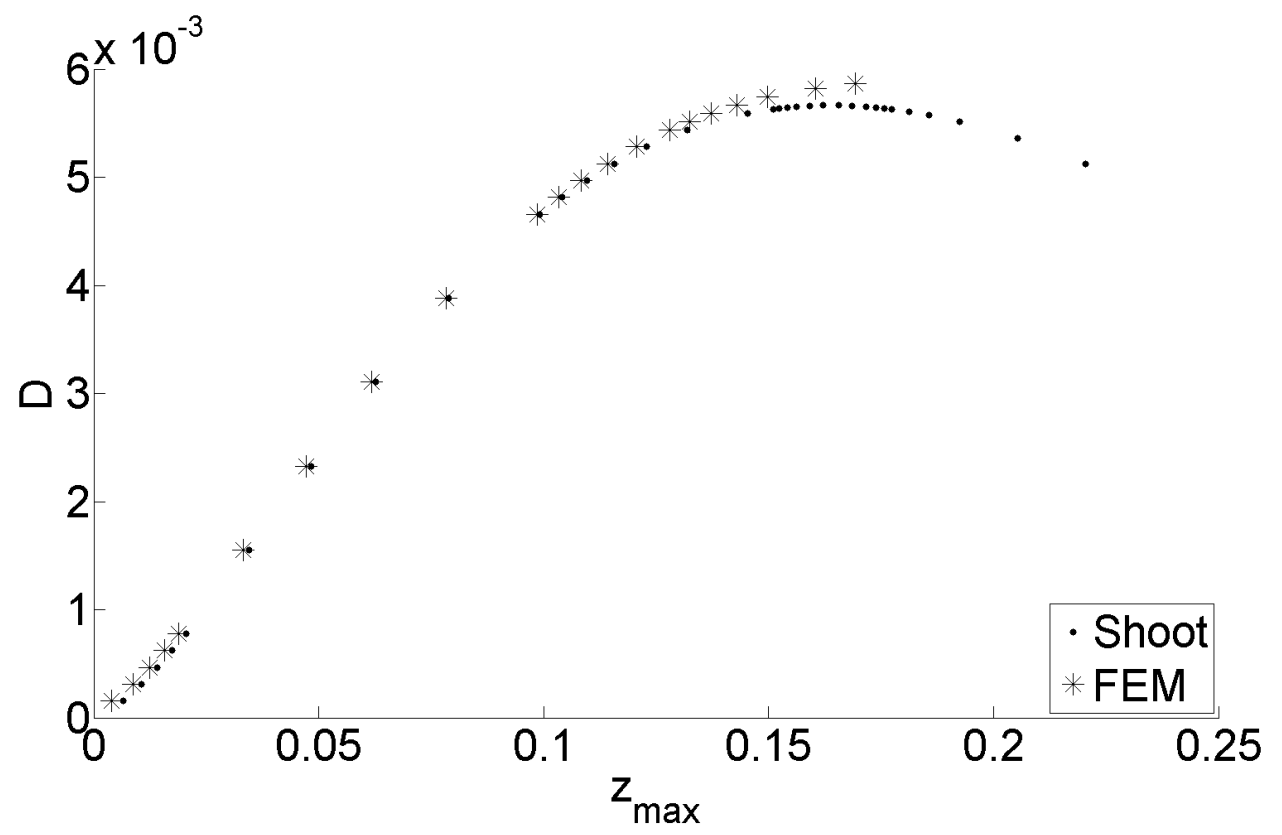

Figure 5.16: Comparison of the $z$-displacement at the center of the film for the two methods as a function of dipole strength. This particular FEM result used a film thickness of $h=$ $70 \mu \mathrm{m}$.

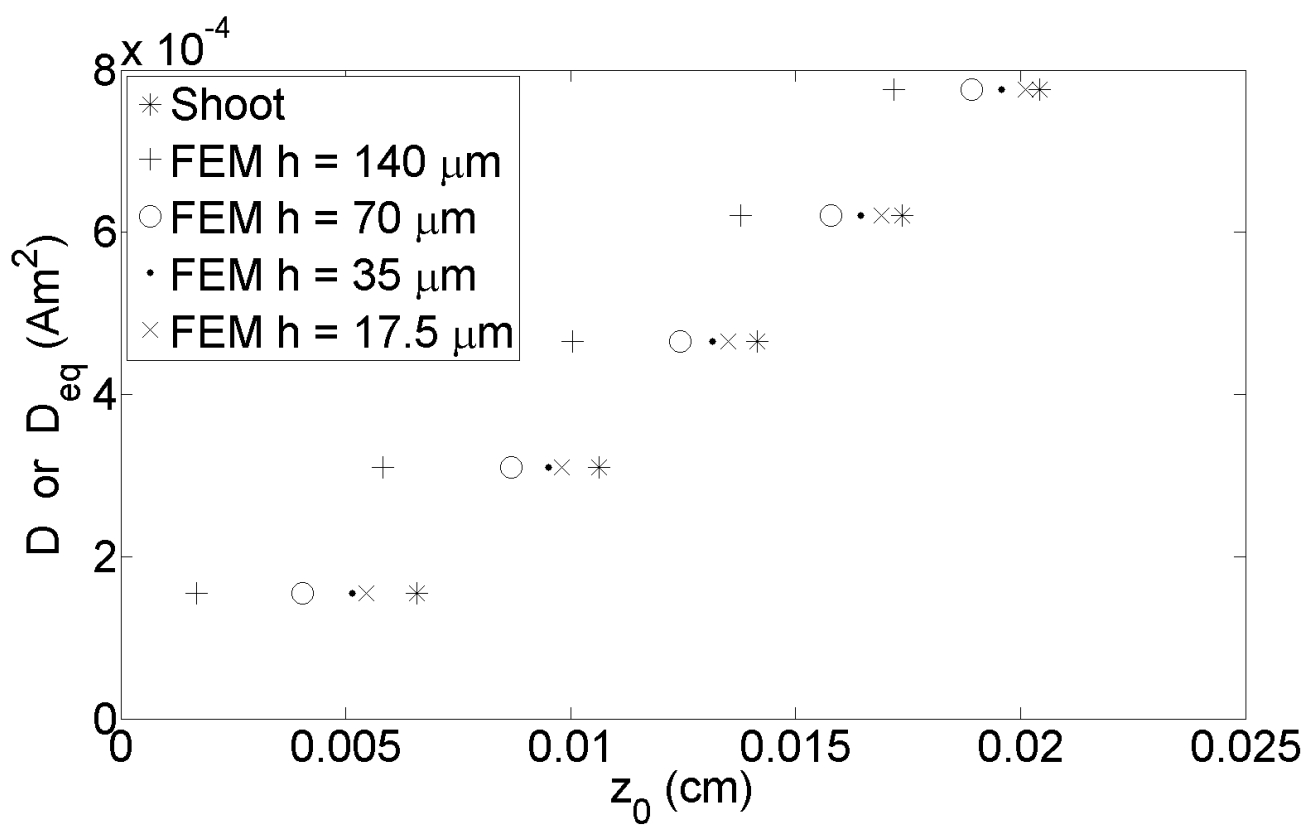

Figure 5.17: Comparison of the FEM results, with different film thicknesses, to the shooting method. 


\subsection{Finite Difference Method}

To test our finite difference method before applying it the a more complicated material model described in Chapter 6, we will apply it to the weakly magnetized material model and compare the results to the shooting method described in Section 5.1.

We will follow the procedure in Chapter 4 to solve for the equilibrium position of the deformed film by solving 4.15 (cf. 3.81) with $\Delta p=0$. We discretize the circular reference mid-plane $(\Omega)$ using the "butterfly" mesh described in Chapter 4

We first need to solve for the in plane stress $(\mathbf{P 1})$ at zone centered points. Solving for the 2D in-plain deformation gradient at each zone-centered point with equation 4.13 and then solve for the in-plane right Cauchy Green tensor at zone-centered points:

$$
\mathbf{c}=\mathbf{f}^{t} \mathbf{f}
$$

We solve the eigenvalue problem of this tensor where the eigenvectors are the reference principal directions $\left(\mathbf{u}_{\alpha}\right)$ and the eigenvalues are the square of the principal stretch $\left(\lambda_{\alpha}\right)$, thus

$$
\begin{aligned}
& \lambda_{1}^{2}=\frac{\operatorname{tr}(\mathbf{c})+\left\{[\operatorname{tr}(\mathbf{c})]^{2}-4 \operatorname{det}(\mathbf{c})\right\}^{\frac{1}{2}}}{2} \\
& \lambda_{2}^{2}=\frac{\operatorname{tr}(\mathbf{c})-\left\{[\operatorname{tr}(\mathbf{c})]^{2}-4 \operatorname{det}(\mathbf{c})\right\}^{\frac{1}{2}}}{2} \\
& \mathbf{u}_{\alpha}=\left[\begin{array}{c}
\frac{1}{\left\{1+\left(\frac{c_{11}-\lambda_{\alpha}^{2}}{c_{12}}\right)\right\}^{\frac{1}{2}}} \\
\left\{1+\left(\frac{c_{12}}{c_{11}-\lambda_{\alpha}^{2}}\right)\right\}^{\frac{1}{2}}
\end{array}\right]
\end{aligned}
$$

From this the current principal stretch directions are calculated

$$
\mathbf{v}_{\alpha}=\mathbf{f u}_{\alpha}
$$

These principle stretches and directions are used in equation 5.19 to determine the stress at zone centered points. We then solve for the divergence of the stress at the nodes centered points with 4.2 and 4.4 depending on the nearest neighbors. The calculation of $R_{k}$ is simplified as the $\left(\operatorname{grad} \mathbf{h}_{a}\right)_{k i}$ and $m_{i}=f_{i \alpha} M_{\alpha}$ can be solved for directly thus, equation $4.8_{3}$ is:

$$
R_{k}=\frac{H}{\left(\ell^{i, j}\right)^{6}}\left\{\left(\left(\ell^{i, j}\right)\left(\mathbf{a}^{i, j}\right) \cdot \mathbf{k}\right) \mathbf{k}-\left[1+4\left(\left(\mathbf{a}^{i, j}\right) \cdot \mathbf{k}\right)^{2}\right]\left(\left(\ell^{i, j}\right)\left(\mathbf{a}^{i, j}\right)\right\}\right.
$$

where $\ell$ and $\mathbf{a}$ are solved for from the current position with equation 2.36. 
Thus we use equation 4.19 to find the velocity of each node for the first time step then equation $4.17_{1}$ for further time steps and then equation $4.17_{2}$ to solve for the new deformed position. We then move forward in time until the deformation has reached equilibrium. Once the maximum norm for all nodes is below a defined tolerance 4.20 we stop advancing in time and declare the deformed position the equilibrium position. We also check that the maximum velocity is approaching zero and that the maximum $z$-displacement reaches a constant.

\subsubsection{Results}

Results for the finite difference method are for films with the following properties and dimensions: radius of the film $\left(r_{a}\right)$ of $0.317 \mathrm{~cm}$; film shear modulus $(G)$ of $0.25 \mathrm{MPa}$; MooneyRivlin parameter $\delta$ of 0.9 ; magnetic susceptibility $(\chi)$ of 2.5 ; film thickness $(h)$ of $70 \mu \mathrm{m}$; dipole height $z_{d}$ of $0.5 \mathrm{~cm}$; and a variety dipole strengths $(D)$.

A mesh refinement study is performed, the four finest meshes considered are shown in Figure 5.18. The mesh refinement study is performed at a dipole strength of $D=0.00466 \mathrm{Am}^{2}$ and using the relation between the two "butterfly" mesh quantities $N_{r}=\left(N_{c}-1\right) / 4$. The $z$-displacement is compared for each mesh, see Figure 5.19. It is seen that a mesh of $N_{c}=129$ and $N_{c}=65$ produce center displacements that are $0.017 \%$ different. All further results will be for a mesh size of $N_{c}=65$ and $N_{r}=16$.

To check to make sure a small enough norm tolerance $(t o l)$ is used we look at the velocity and center $z$-displacement of the film over "time" to make sure that the velocity is approaching zero and the $z$-displacement is approaching a constant. See Figures 5.20 and 5.21 for these plots.

Figure 5.22 shows the 3D deformation for a dipole strength of $D=0.00466 \mathrm{Am}^{2}$. Where Figure 5.23 shows the axisymmetric deformation at $\mathrm{x}=0$ for a variety of dipole strengths, no comparison to the shooting method is made as the displacement curves fall directly on top of each other and no difference can be seen. Figure 5.24 shows a comparison of the films center $z$-displacement for the shooting method and finite difference method. It is seen that the both methods agree, thus validating the deformation portion of the finite difference method with dynamic relaxation. 


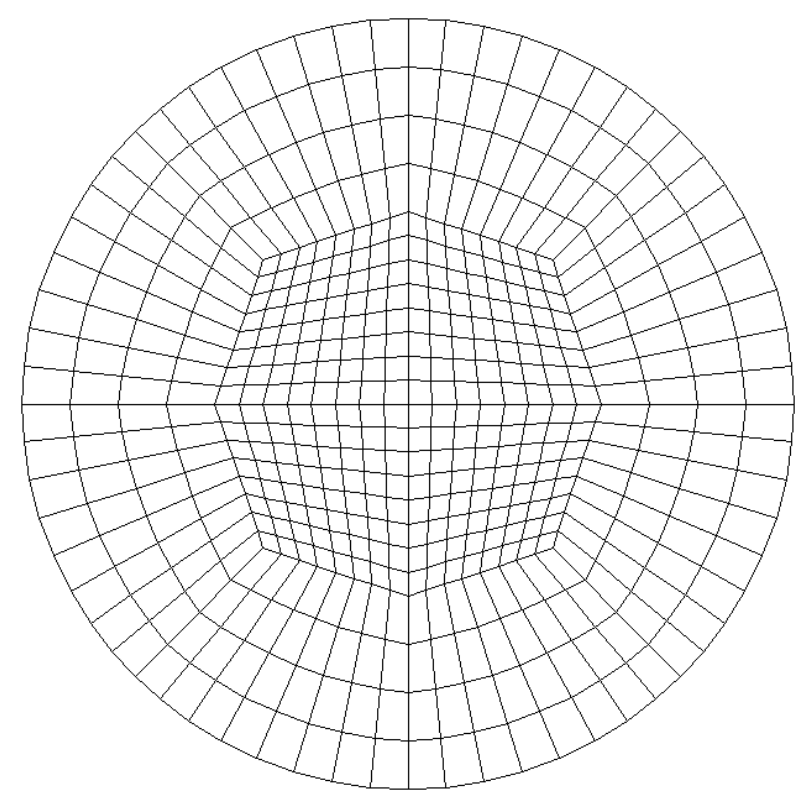

(a) $N_{c}=17$ and $N_{r}=4$

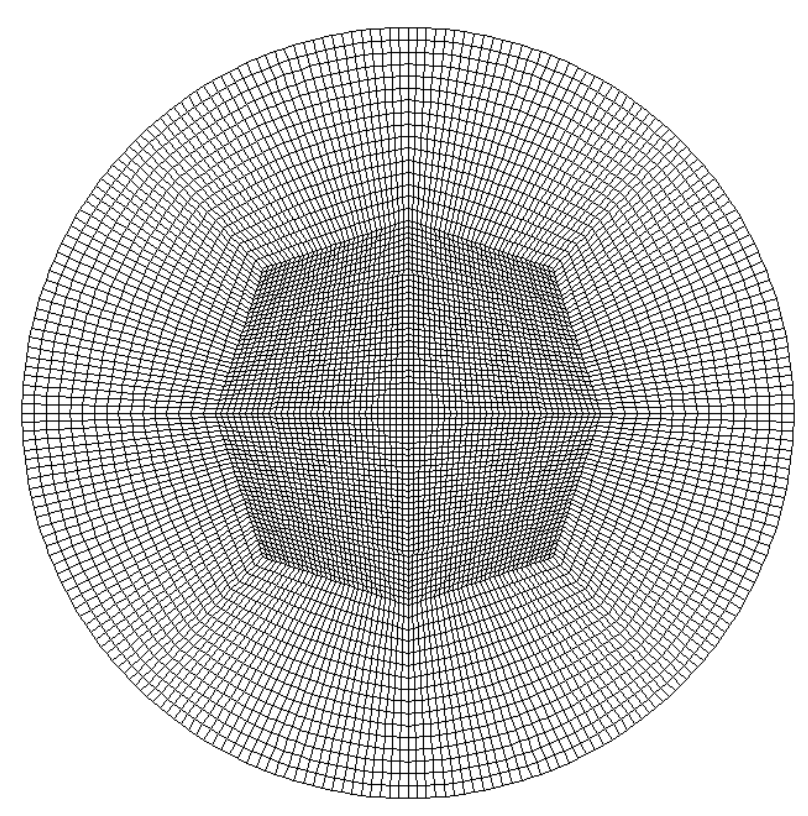

(c) $N_{c}=65$ and $N_{r}=16$

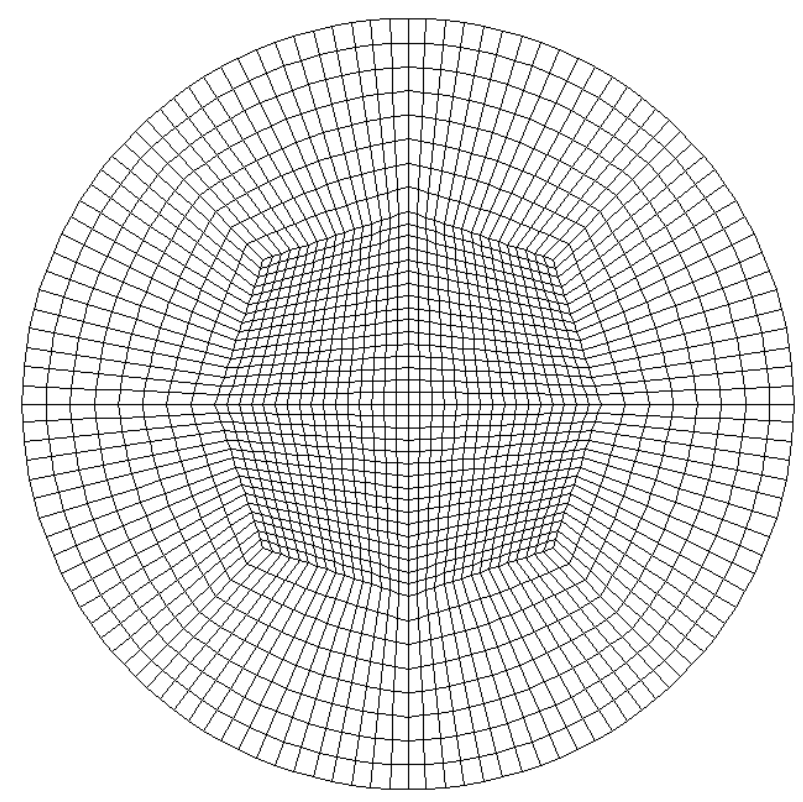

(b) $N_{c}=33$ and $N_{r}=8$

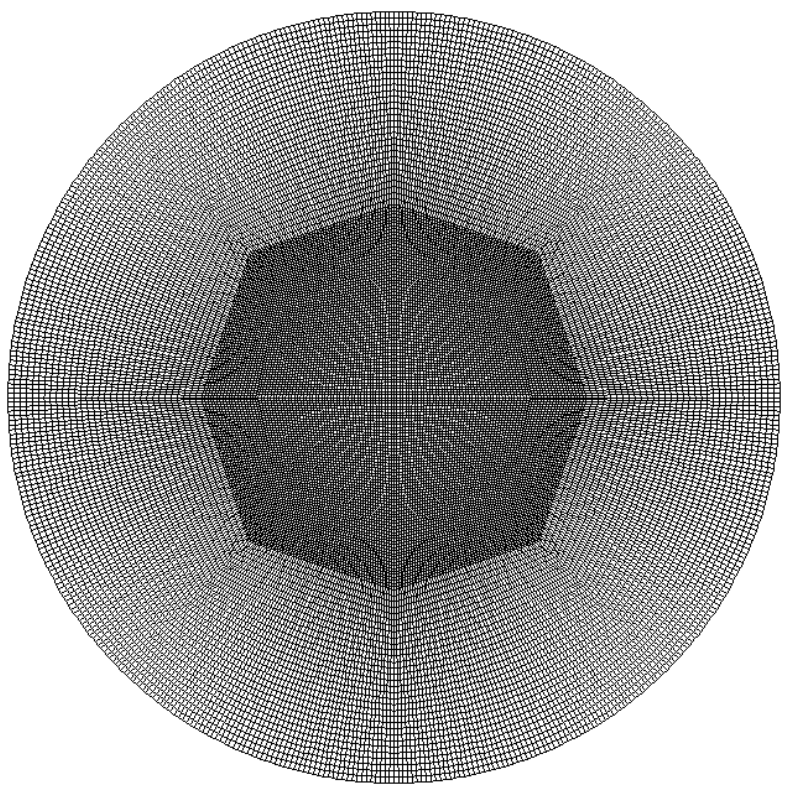

(d) $N_{c}=129$ and $N_{r}=32$

Figure 5.18: "Butterfly" meshes considered 


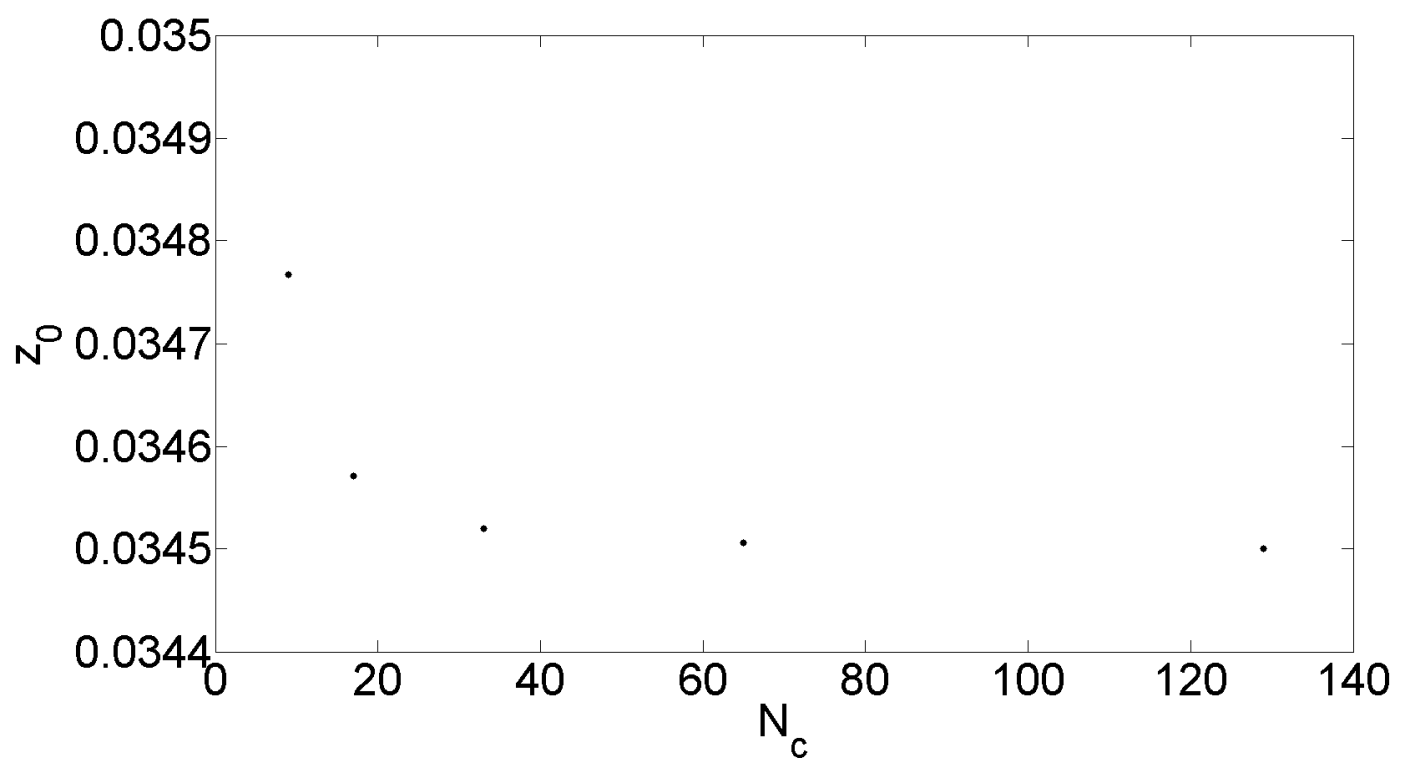

Figure 5.19: Mesh Refinement study at $D=0.00466 \mathrm{Am}^{2}$

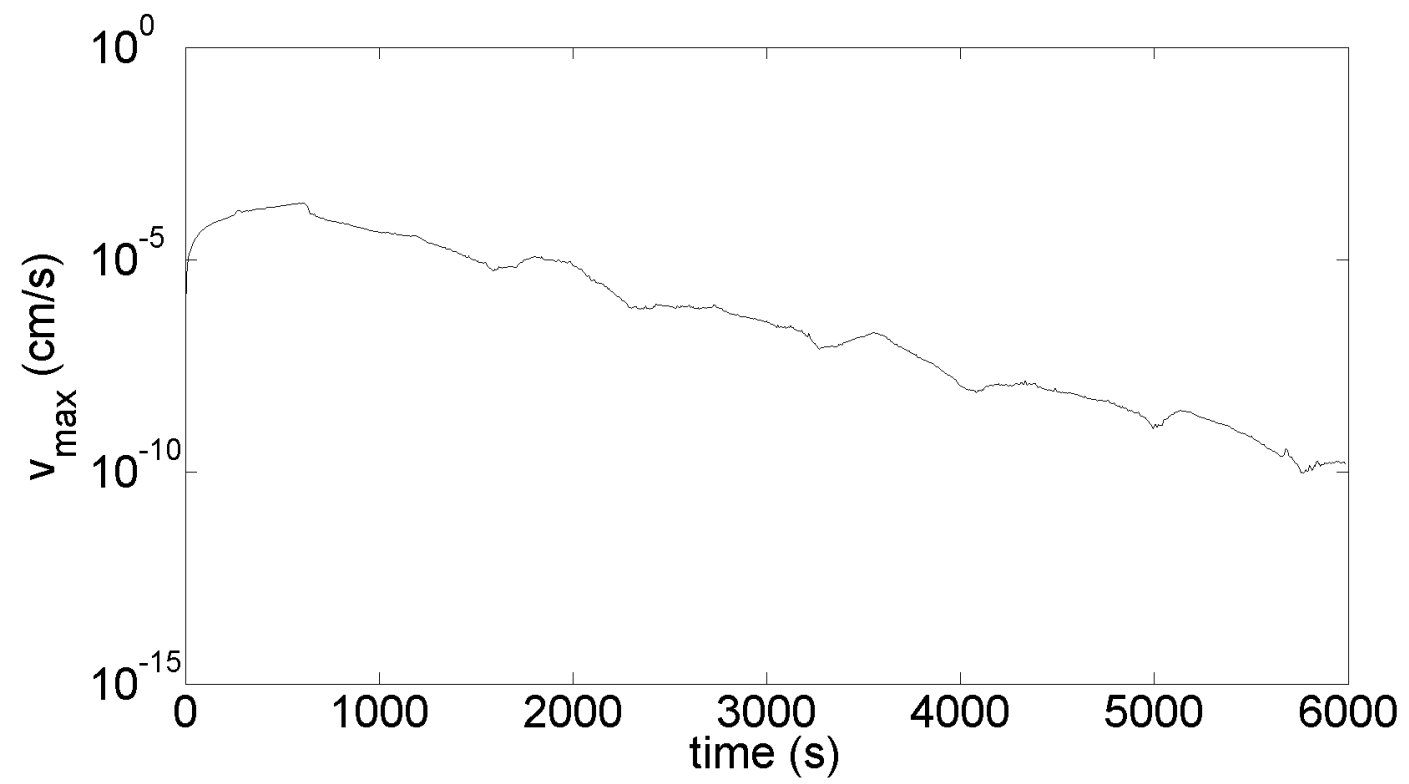

Figure 5.20: Maximum nodal artificial velocity vs artificial time for $D=0.00466 \mathrm{Am}^{2}$. 


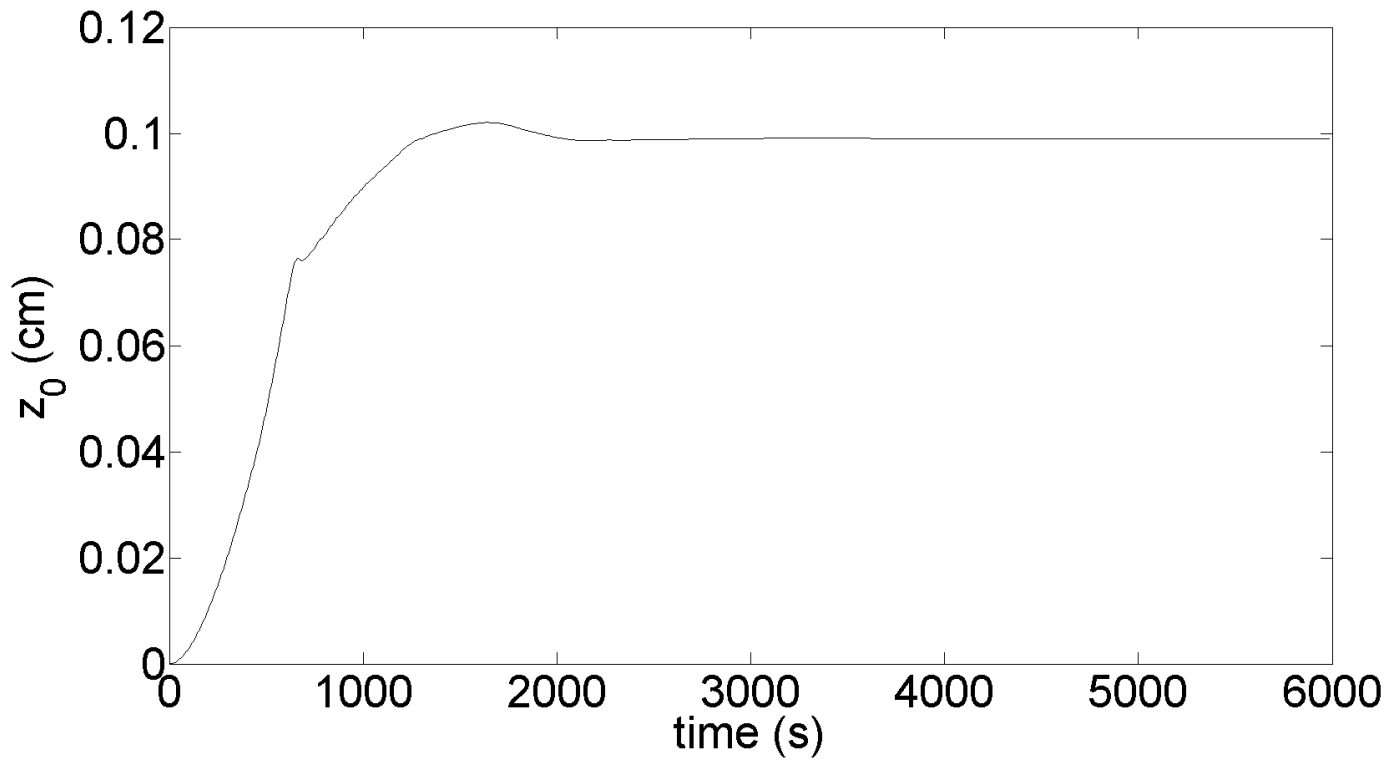

Figure 5.21: Film center height displacement vs. artificial time for $D=0.00466 \mathrm{Am}^{2}$. 


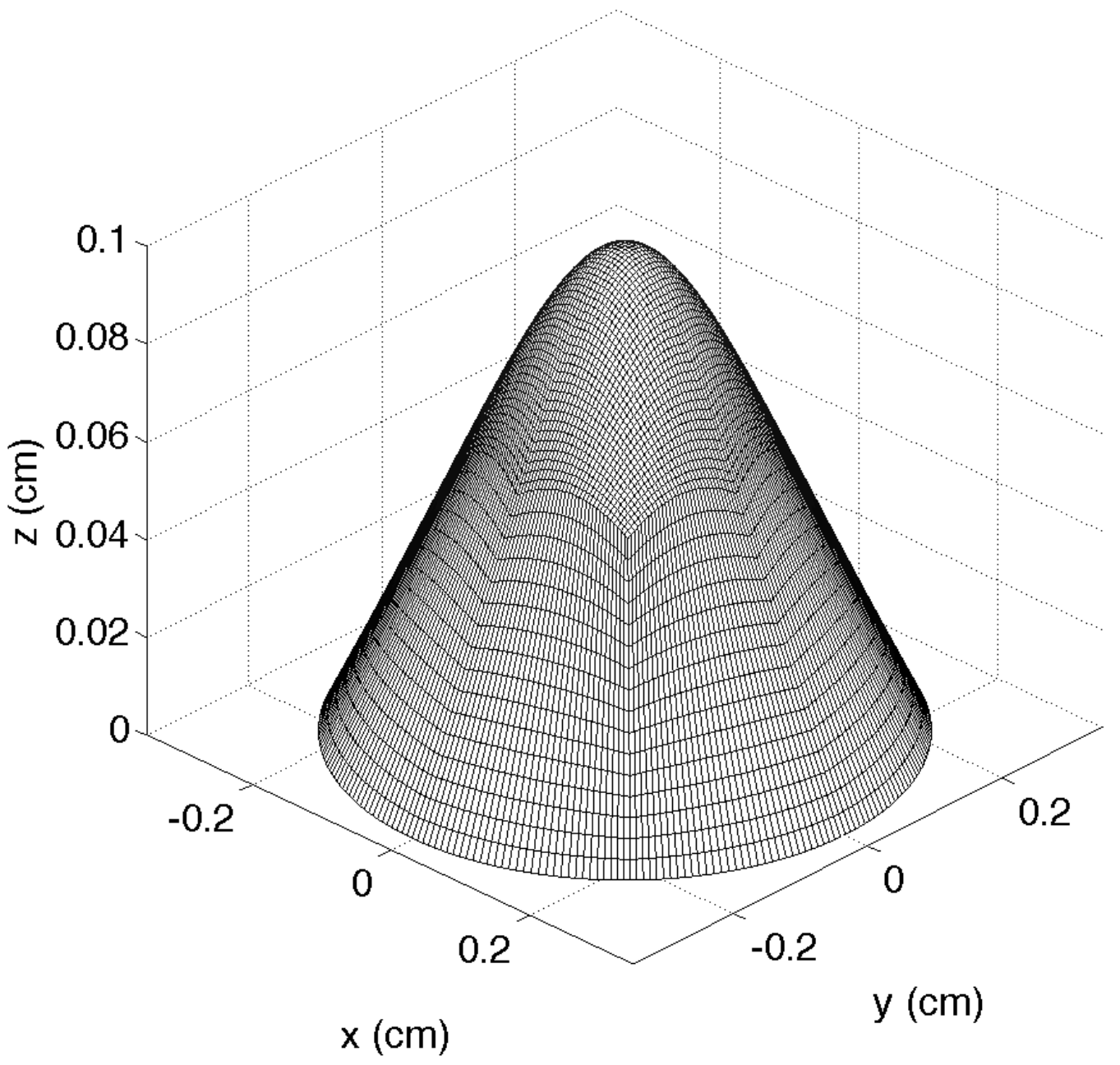

Figure 5.22: 3D deformation of film using finite difference for $N_{c}=64$ and $D=0.00466 \mathrm{Am}^{2}$. 


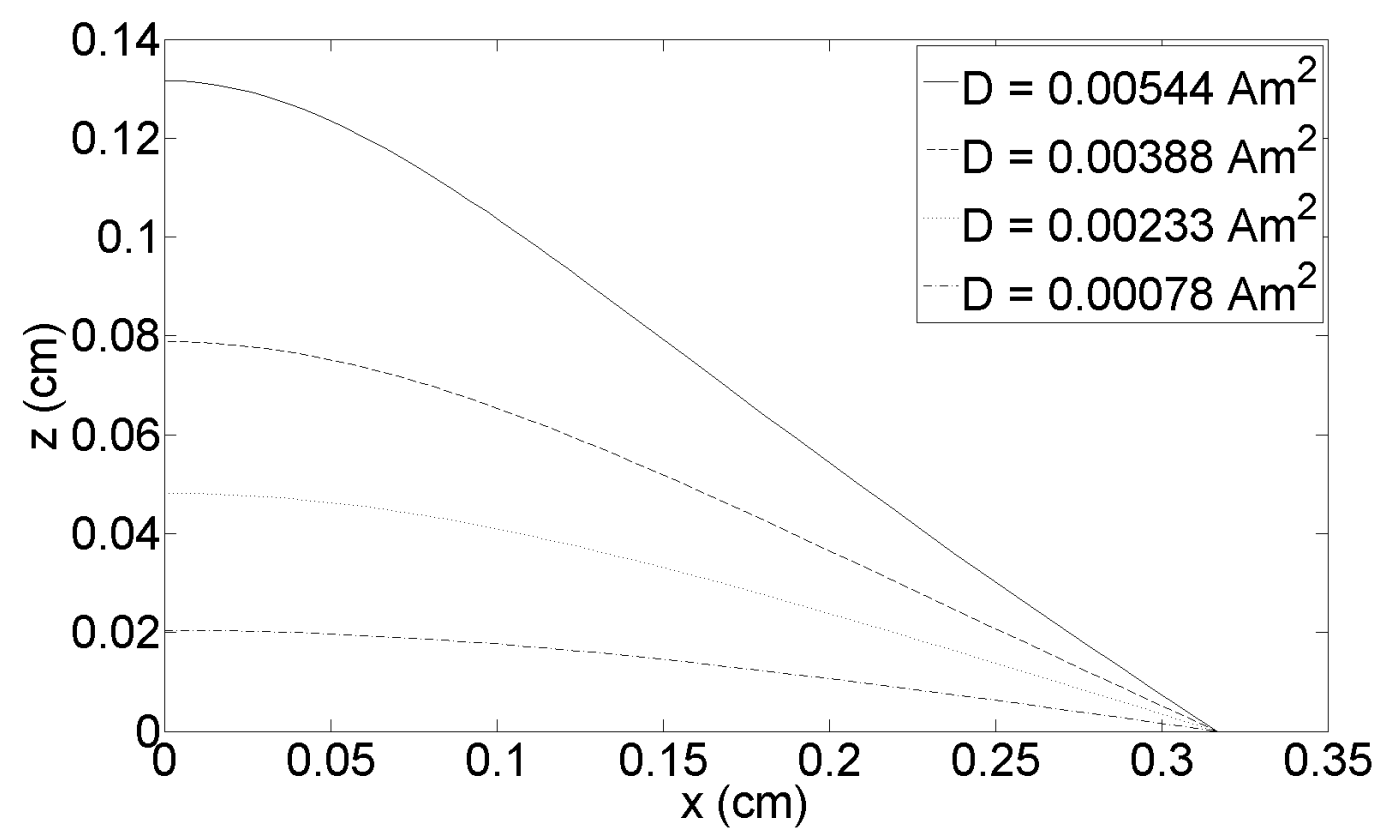

Figure 5.23: Cross-sectional deformation for a variety of dipole strengths for the finite difference method.

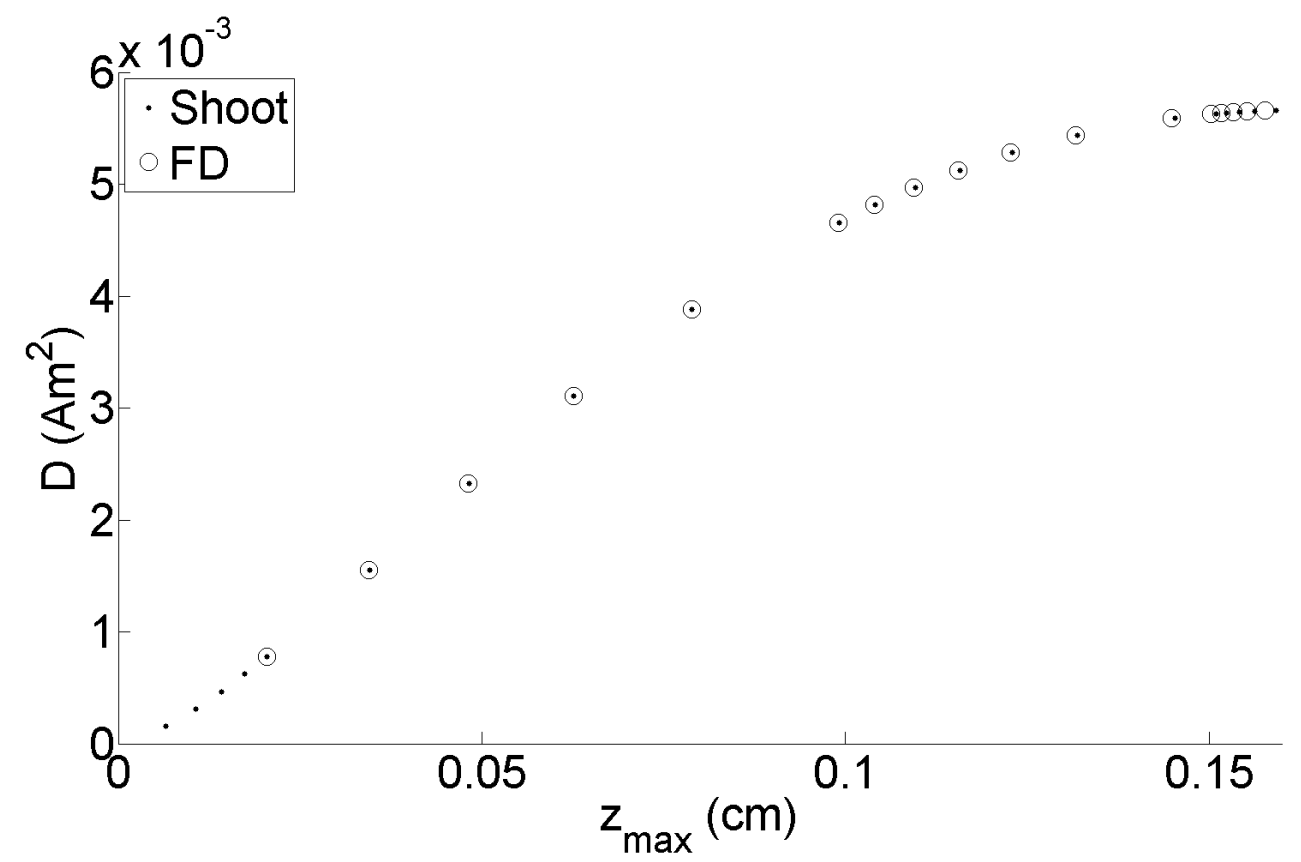

Figure 5.24: Comparison of the $z$-displacement at the center of the film for the membrane model solved with the shooting method (Shoot) and finite difference (FD). 


\section{Chapter 6}

\section{Magnetoelastic Strain-Energy Function}

In this chapter we use the material model proposed by Kankanala \& Triantafyllidis in [20], where here the magnetization and mechanical stress are closely coupled and is an extension of the classical Mooney-Rivlin. We apply this material model to the finite difference method developed in Chapter 4 to solve for equilibrium based on the membrane model of Chapter 3. This material model, in the form of Section 2.6, is defined with the strain-energy function by $[20]$

$$
\begin{aligned}
U=\frac{G}{2}\{ & {\left[C_{10}+C_{11} \frac{J_{1}}{\left(\bar{M}_{s}\right)^{2}}\right]\left(I_{1}-3\right)+\left[C_{20}+C_{21} \frac{J_{1}}{\left(\bar{M}_{s}\right)^{2}}\right]\left(I_{2}-3\right) } \\
& \left.+C_{01} \frac{J_{1}}{\left(\bar{M}_{s}\right)^{2}}+C_{02} \frac{J_{1}}{\left(\bar{M}_{s}\right)^{2}}+C_{01}^{*}\left[\cosh \left(\frac{J_{1}}{\left(\bar{M}_{s}\right)^{2}}\right)-1\right]\right\}
\end{aligned}
$$

where

$$
J_{1}=I_{5}-I_{1} I_{4}+I_{2} I_{6} \quad \text { and } \quad J_{2}=I_{6}
$$

in which $\operatorname{det} \mathbf{F}=1$ has been imposed. Here $G$ is the ground-state shear modulus, $\bar{M}_{s}$ is the saturation value of magnetization per unit volume, and the $C_{i j}$ are dimensionless constants. Numerical values of $G, C_{i j}$ and $\mu_{0} \bar{M}_{s}$ are given in Table 2 of [20] and [21], where $\mu_{0}$ is the free-space permeability. The symbols $J_{1,2}$ are used in [20] to denote invariants based on magnetization per unit mass. These are recovered on dividing our invariants by $\rho_{\kappa}^{2}$, and 6.1 takes this adjustment into account. Further, we have used $2.124_{2}$ and 2.128 to express the invariants adopted in [20], here based on magnetization per unit volume, in terms of the $I_{k}$. In [20] it is claimed that 6.1 satisfies $2.116_{1}$ without qualification. This comports with the fact that the standard Mooney-Rivlin model satisfies the purely mechanical strong ellipticity condition at all deformations [27]. Inequality $2.116_{2}$ was also shown in [20] to be satisfied over a substantial range of strain. 


\subsection{Results}

In this section we discuss the results of some numerical experiments. All examples pertain to a membrane with a thickness $h=50 \times 10^{-3} \mathrm{~mm}$. This is used in place of $\epsilon$ in the formula 3.43 for the self field, which was derived using a scheme where $\epsilon$ is interpreted as (dimensionless) thickness. The mass density is $\rho=1750 \mathrm{~kg} / \mathrm{m}^{3}$; the free-space permeability is $\mu_{0}=4 \pi \times$ $10^{-7} \mathrm{~N} / A^{2}$ [23]; and the dipole source is centrally located above the plane at $\mathbf{y}_{d}=(8$ $m m) \mathbf{k}$. Material parameters are taken to be those suggested in [21]. Thus, the saturation magnetization is $\bar{M}_{s}=\mu_{0} / 2$, the shear modulus is $\mu=1.0 \times 10^{6} \mathrm{~N} / \mathrm{m}^{2}$, and the remaining parameters in 6.1 are $C_{10}=1.0, C_{20}=0.625, C_{11}=0.0791, C_{21}=0.0, C_{01}=\beta / 6$ and $C_{02}=\beta / 2$, where $\beta=\mu_{0} \bar{M}_{s}^{2} / 2$.

\subsubsection{Square Geometry}

The first referential geometry we look at is a square, with each side being $8.0 \mathrm{~mm}$. In order to see if the number of nodes used in simulations is large enough to capture the actual equilibrium deformation, a refinement study was performed for a dipole of strength $D=160 \times 10^{-6} \mathrm{Am}^{2}$ where the center displacement of the membranes is compared, see Figure 6.1. Where the number of nodes in the $x$ and $y$ direction $(N)$ is increased the center

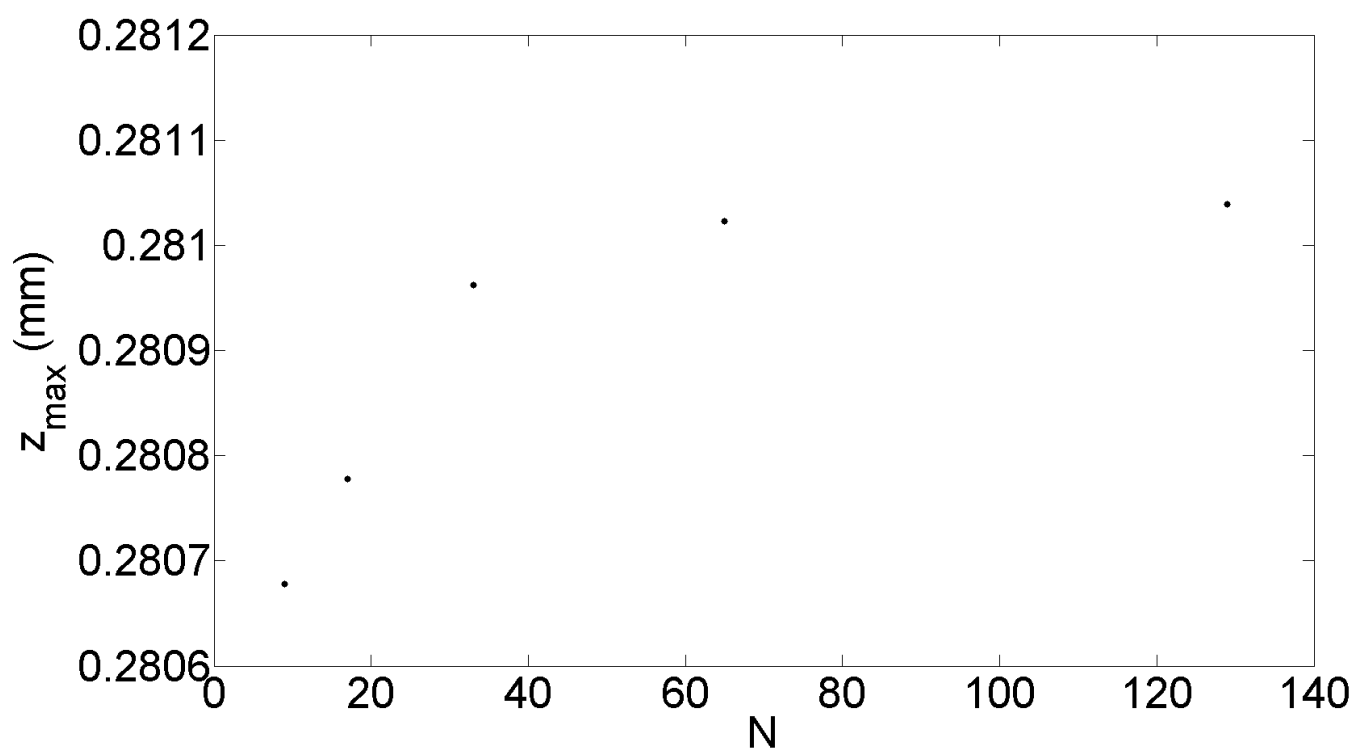

Figure 6.1: Square membrane center displacement for a variety of meshes defined by $N$ for $D=160 \times 10^{-6} \mathrm{Am}^{2}$ and zero pressure.

displacement asymptotically approaches a constant value. At $N=33$ the results produce 
a $0.0274 \%$ error when the most refined simulation $(N=129)$ is assumed to be the exact solution. All further simulations of the square membrane will be performed with $N=33$.

In order to validate that the choice of tol in 4.20 is small enough, we look at the speed and maximum displacement of all nodes as time progresses to see if the simulations are reaching steady state. See Figure 6.2 and 6.3 for the maximum nodal displacement and speed versus

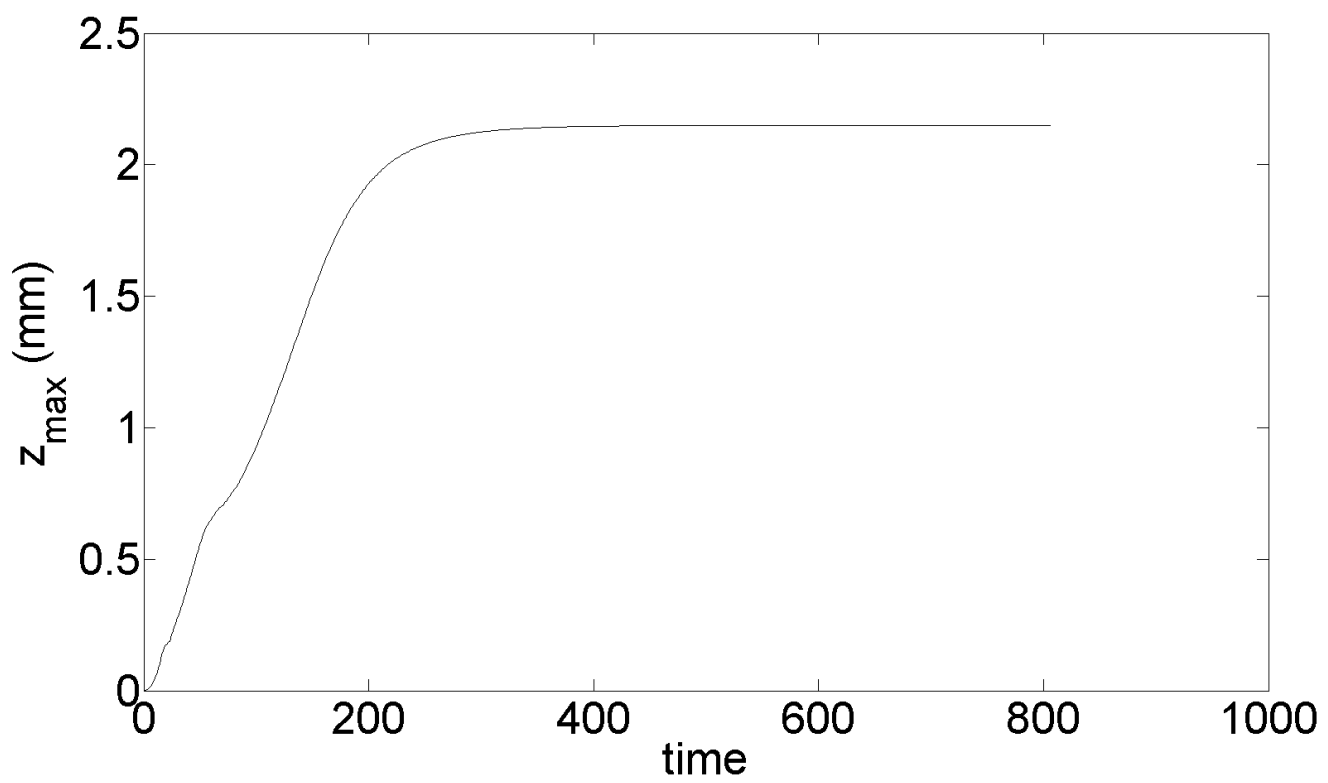

Figure 6.2: Maximum nodal $z$-displacement for $D=160 \times 10^{-6} \mathrm{Am}^{2}$ for a square membrane.

time associated with the dynamic relaxation, respectively. As time progresses the maximum nodal displacement approaches a constant and the speed approaches zero. Thus we conclude that the tol was small enough and that an equilibrium was reached.

Figure 6.4 depicts the deformation of the membrane under zero pressure in response to a dipole of strength $D=160 \times 10^{-6} \mathrm{Am}^{2}$ (cf. 2.35). The vertical and in-plane dimensions are scaled differently to aid in visualization.

We have used the data generated by the simulation, together with 3.13 , to verify that the three-dimensional principal stretches of the membrane surface are well within the limits required for the validity of $2.116_{2}$. The referential in-plane magnetization field $\mathbf{M}$ is shown in Figure 6.5. This field is consistently directed toward the center of the membrane, where differentiability requires that it diminish its intensity to zero. With this observation of the in-plane magnetization, the constraint 3.35 causes the interaction with the applied field to weaken near the center, resulting in a deformed surface that is relatively flat under the dipole source.

Figure 6.6 shows the variation of the in-plane part, $\mathbf{e}$, of the director field with respect to 


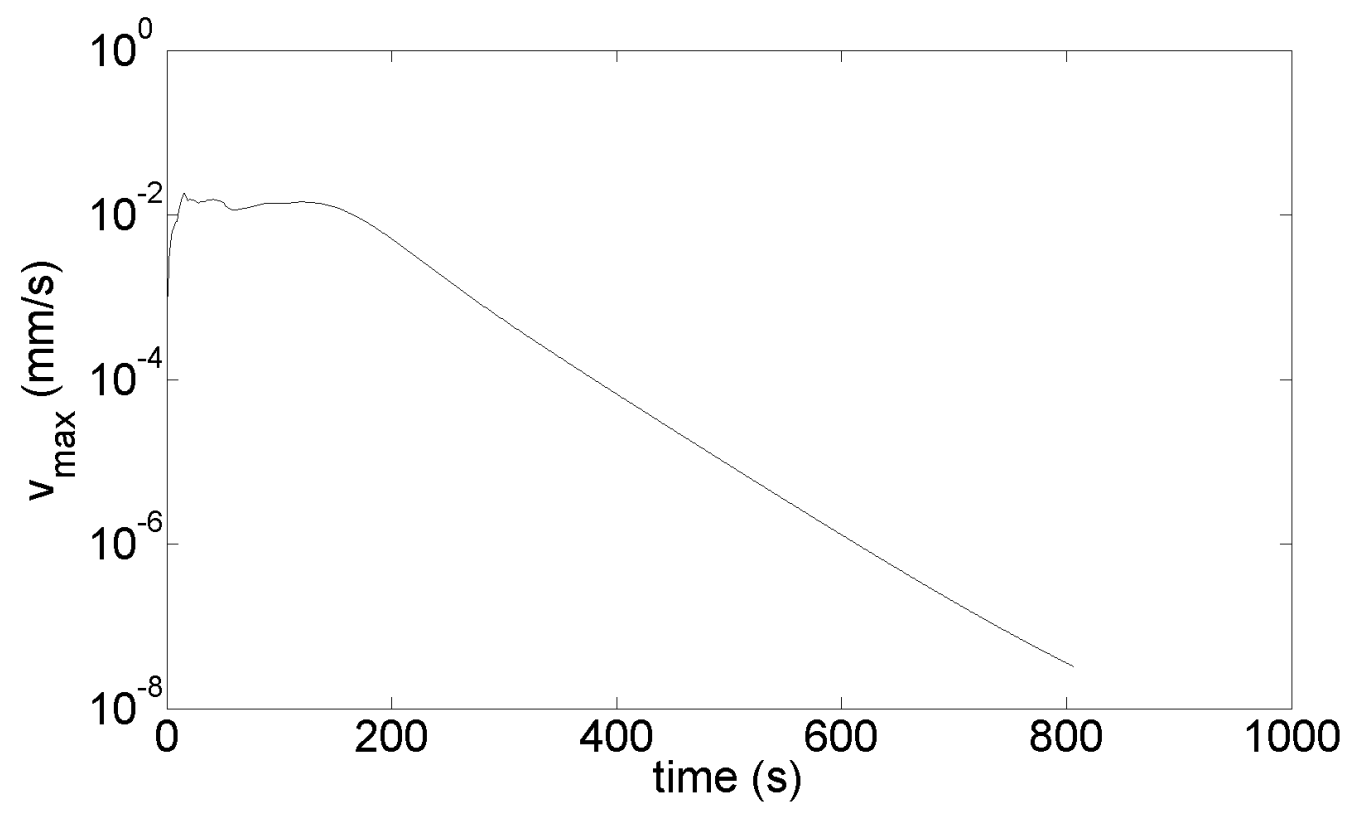

Figure 6.3: Maximum nodal velocity for $D=160 \times 10^{-6} A m^{2}$ for a square membrane.

position on the reference plane. The deformation deviates from Kirchhoff-Love kinematics wherever this is nonzero. This reflects the bias induced by the dipole source at points lying off the dipole axis, causing the director $\mathbf{d}$ on the deformed surface to tilt relative to the tangent plane as the membrane adjusts to the applied field. The effect diminishes near the corners of the membrane where the field is relatively weak, and near the center where the field lines intersect the membrane orthogonally and the associated bias vanishes; in either case the kinematics revert to the Kirchhoff-Love mode.

Figure 6.7 shows the current magnetization $(\mathbf{m})$ on the deformed membrane where 2.104 is used to push the referential magnetization forward.

Figure 6.8 illustrates the self field generated by the membrane, computed post facto using equation 3.43, in a plane of symmetry obtained by fixing a reference coordinate at the value zero. Figure 6.9 illustrates the self field above the deformed membrane.

In order to see how the deformation changes as the dipole strength is changed we look at cross sections of the displacement for a variety of dipole strengths. Figures 6.10 and 6.11 show the cross section deformation at $u_{2}=0.0$ and $u_{1}=u_{2}$, respectively.

Next the effect of uniform pre-stretch of 1.2 is induced by an outward displacement of nodes on the boundary; these are subsequently fixed in the course of the simulation. Cross sections of the displacement with pre-stretch for a variety of dipole strengths are shown in Figures 6.10 and 6.11 for cross sections $u_{2}=0.0$ and $u_{1}=u_{2}$, respectively.

The equilibrium deformation effect of pressure (no pre-stretch) at a fixed inflation pressure $P=6.0 \times 10^{5} \mathrm{~Pa}$ acting on the interior of the membrane; the external pressure is assumed 


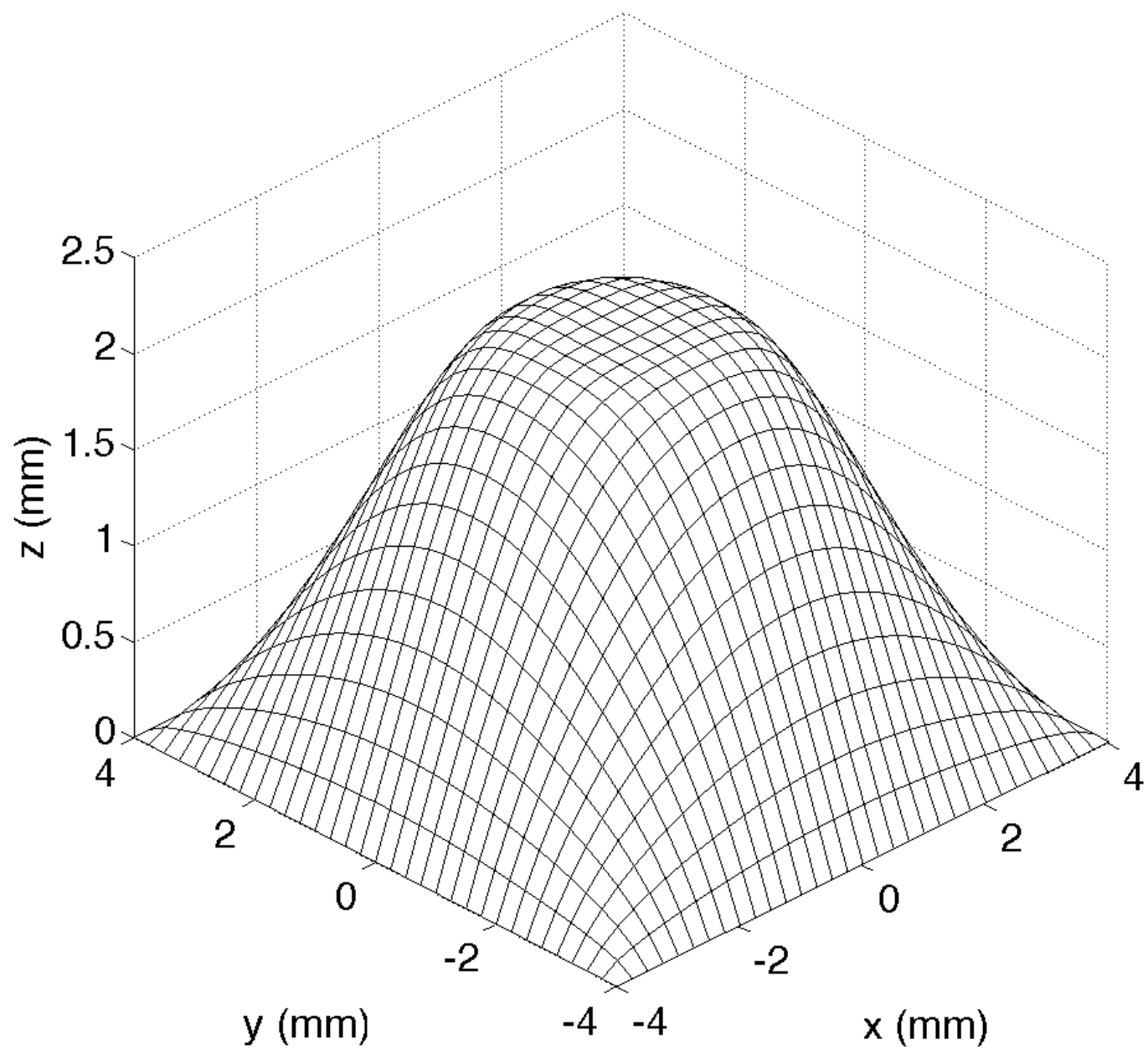

Figure 6.4: Deformed square membrane for $D=160 \times 10^{-6} \mathrm{Am}^{2}$. 


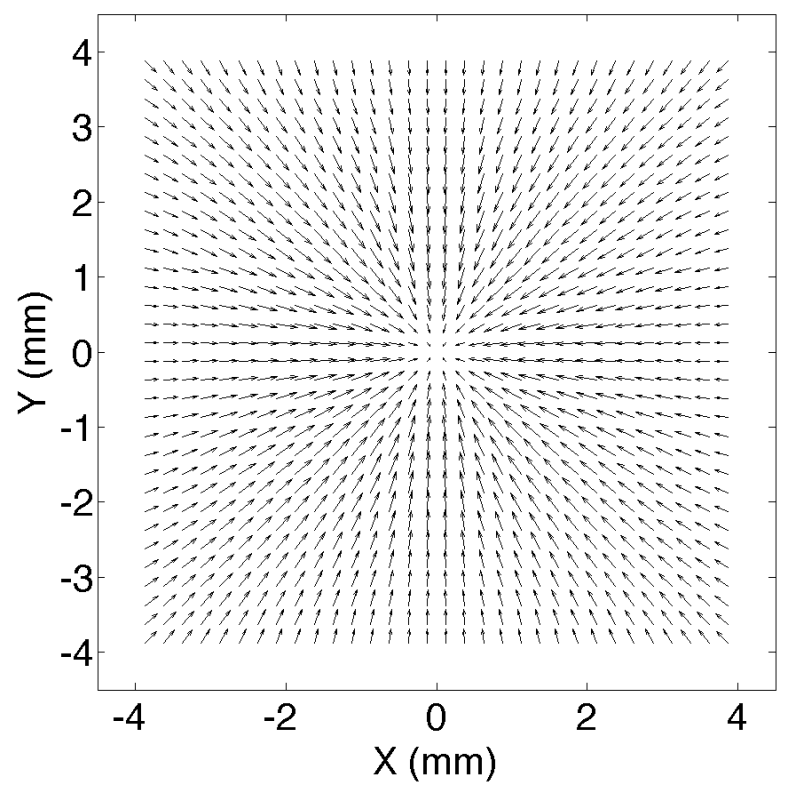

Figure 6.5: Reference magnetization $(\mathbf{M})$ for $D=160 \times 10^{-6} \mathrm{Am}^{2}$ on the reference square membrane.

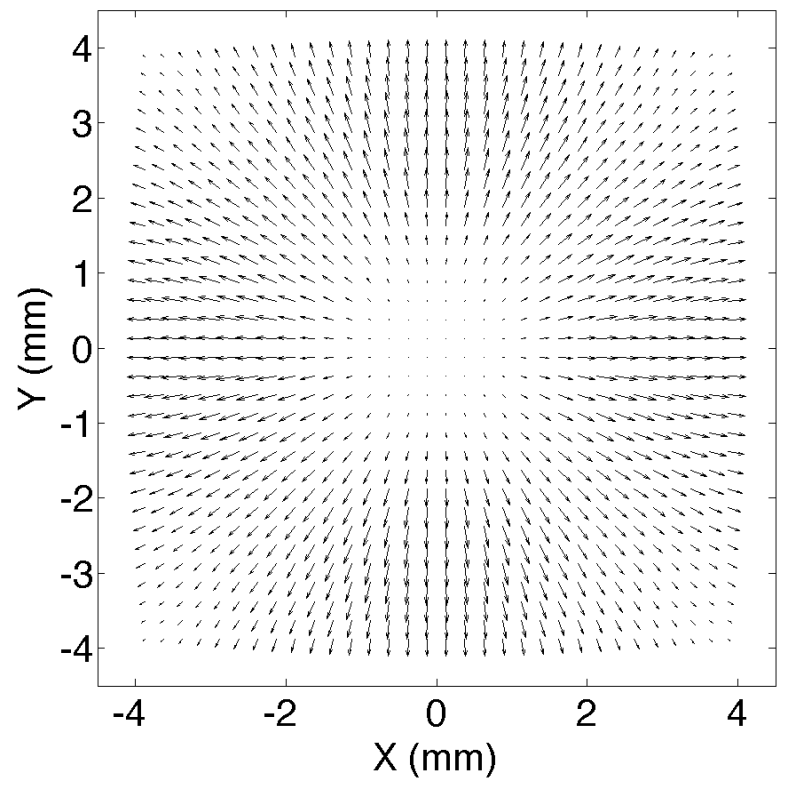

Figure 6.6: In-plane part, e, of director field, at $D=160 \times 10^{-6} \mathrm{Am}^{2}$ for a square membrane. 


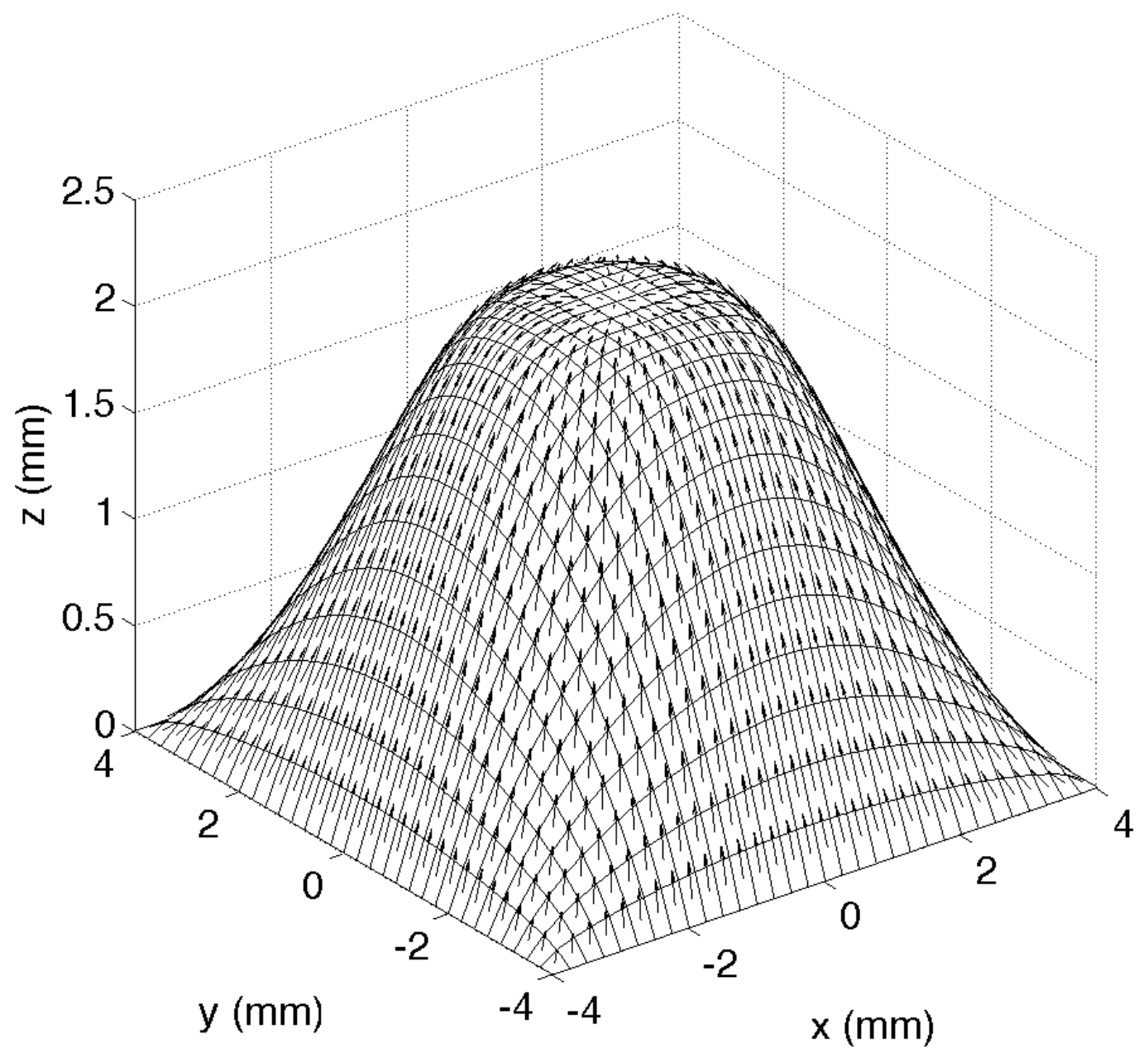

Figure 6.7: Current magnetization $(\mathbf{m})$ for $D=160 \times 10^{-6} \mathrm{Am}^{2}$ on the deformed square membrane. 


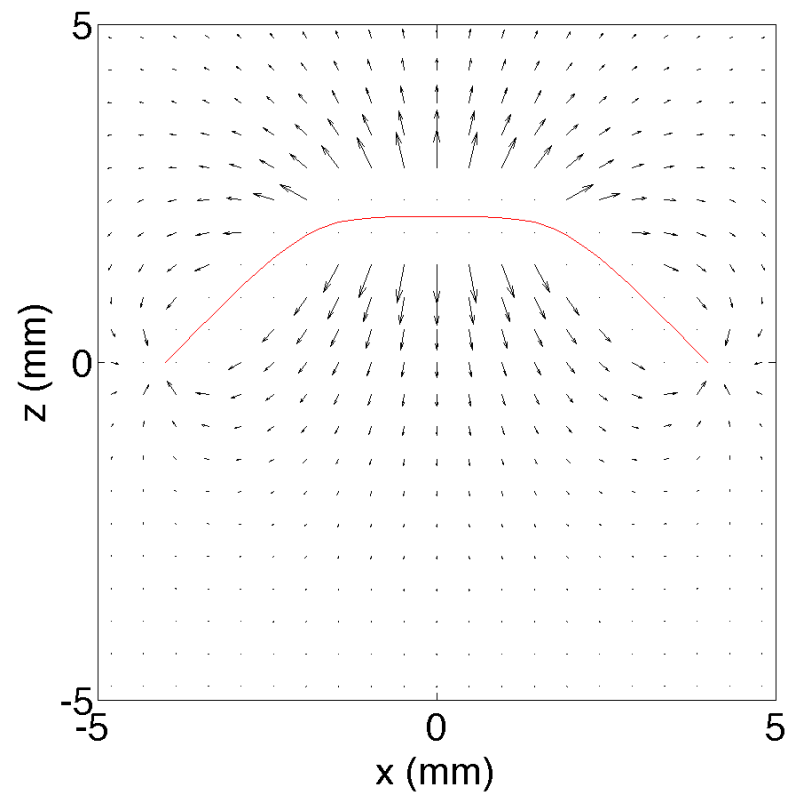

Figure 6.8: Self field in space at $D=160 \times 10^{-6} \mathrm{Am}^{2}$, in the plane defined by $u_{2}=0$ for a square membrane.

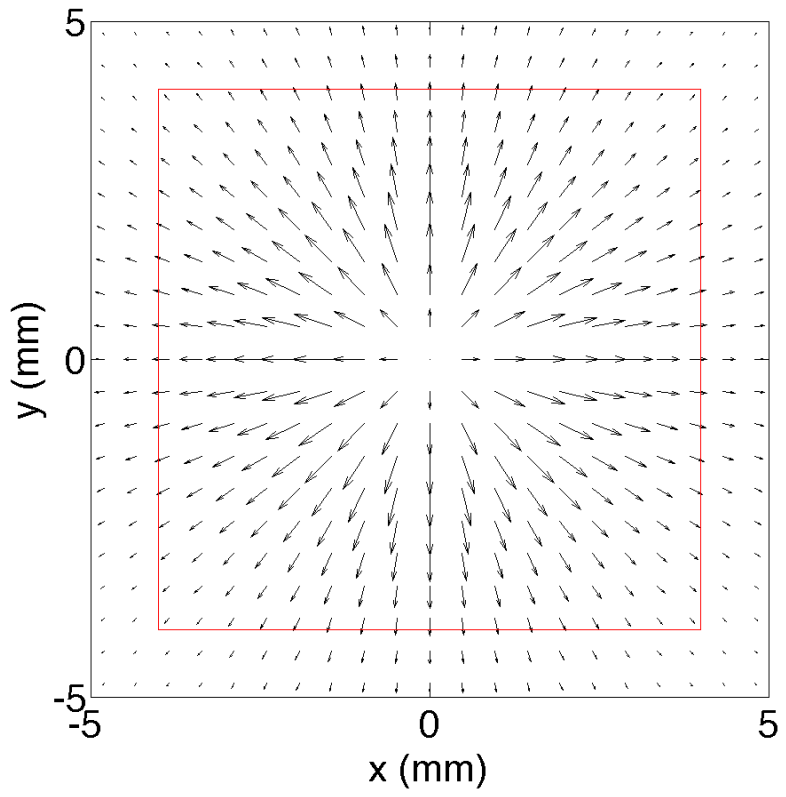

Figure 6.9: Self field in space at $D=160 \times 10^{-6} \mathrm{Am}^{2}$, in the plane defined by $z=3.0 \mathrm{~mm}$ for a square membrane. 


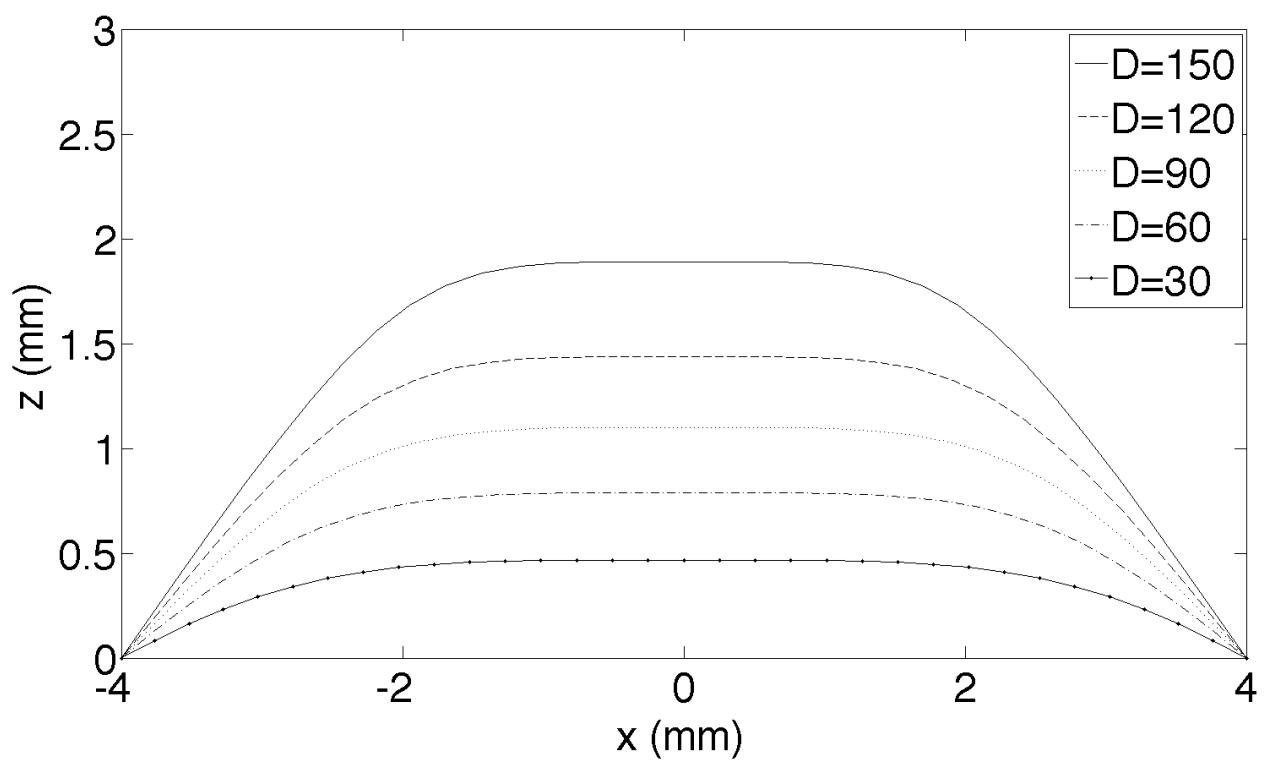

Figure 6.10: Equilibrium displacement of a square membrane for a cross section (at $u_{2}=0$ ) for a variety of dipole strengths, where the values of $D$ given in the figure are multiplied by $\times 10^{-6} \mathrm{Am}^{2}$.

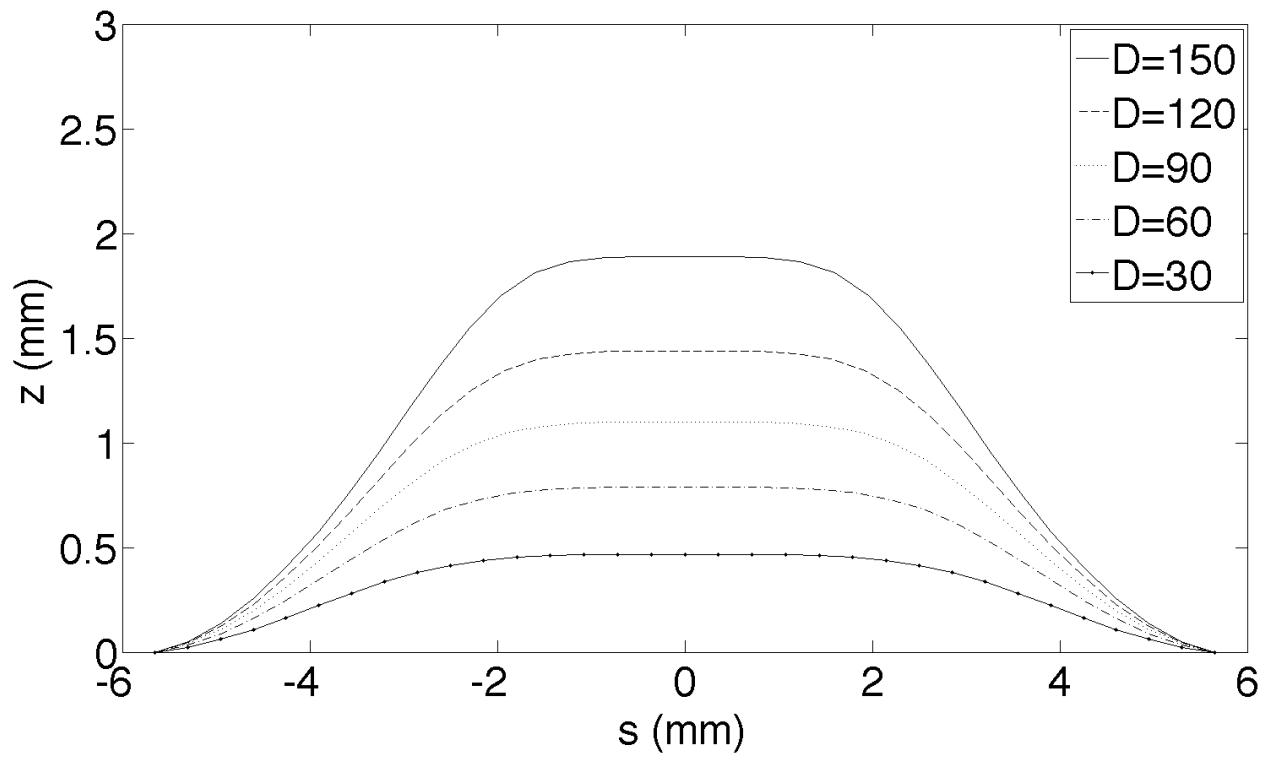

Figure 6.11: Equilibrium displacement of a square membrane for a cross section (at $u_{1}=u_{2}$ ) for a variety of dipole strengths, where the values of $D$ given in the figure are multiplied by $\times 10^{-6} \mathrm{Am}^{2}$. 


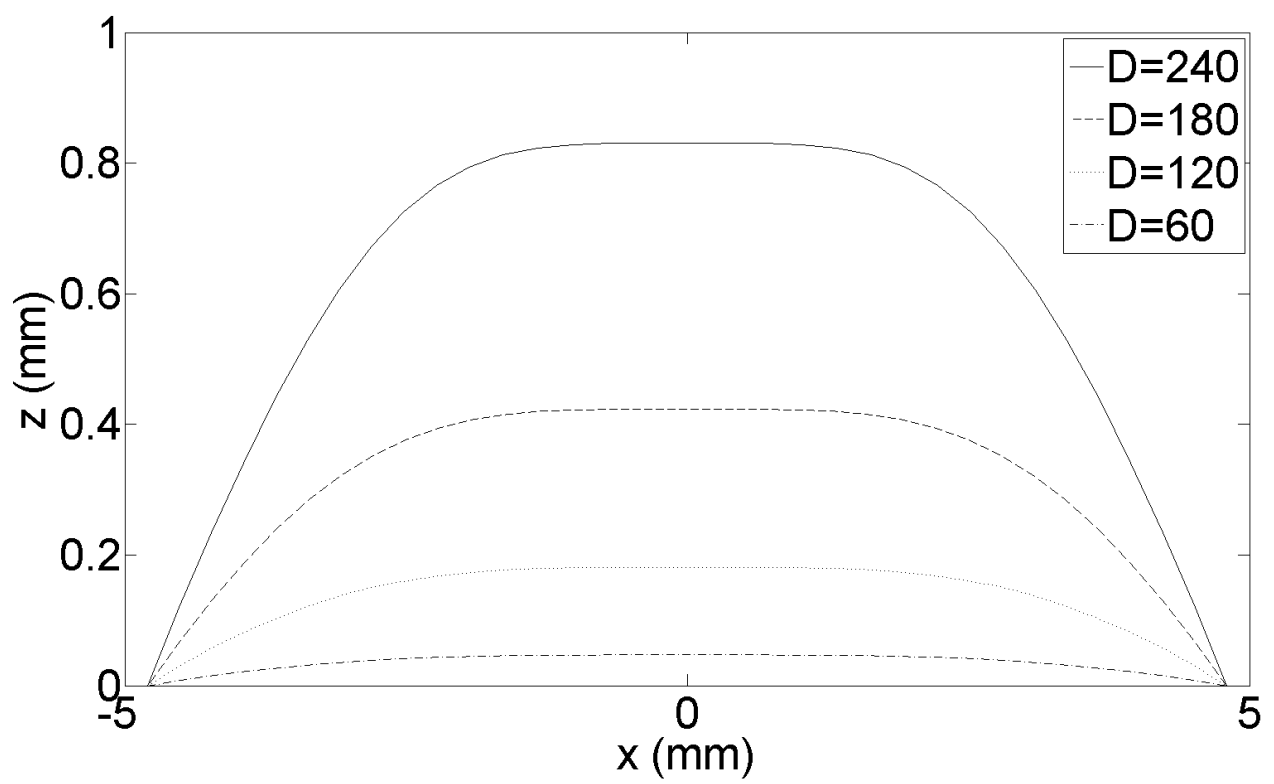

Figure 6.12: Equilibrium displacement of a square membrane for a cross section (at $u_{2}=0$ ) for a variety of dipole strengths with a pre-stretch of 1.2 , where the values of $D$ given in the figure are multiplied by $\times 10^{-6} \mathrm{Am}^{2}$.

to vanish. This is regarded as being supplied by a large reservoir with an opening on the reference plane. Cross sectional deformations are illustrated in Figures 6.14 and 6.15 for cross sections $u_{2}=0.0$ and $u_{1}=u_{2}$, respectively.

Next a volume-dependent pressure in which the product of the pressure and the enclosed volume remains constant, as in an ideal gas at fixed temperature, is examined. The constant is derived by using 3.74 to compute the contained volume generated in response to the fixed pressure at zero field strength. The new pressure is:

$$
P=\frac{P_{0} V_{0}}{V}
$$

where $V$ is the current volume and $P_{0}$ and $V_{0}$ are the pressure and volume at zero field strength, respectively. Cross sections of the deformation are illustrated in Figures 6.16 and 6.17 for cross sections $u_{2}=0.0$ and $u_{1}=u_{2}$, respectively.

Finally, the effects of pre-stretch and pressure are compared in Figure 6.18, in which the height of the deformed surface, at a point on the dipole axis, is plotted against dipole strength. The open circles and crosses correspond to zero applied pressure; the former corresponding to no pre-stretch and the latter to a uniform pre-stretch of 1.2 induced by an outward displacement of nodes on the boundary; these are subsequently fixed in the course of the simulation. Pre-stretch stiffens the membrane dramatically, resulting in a 


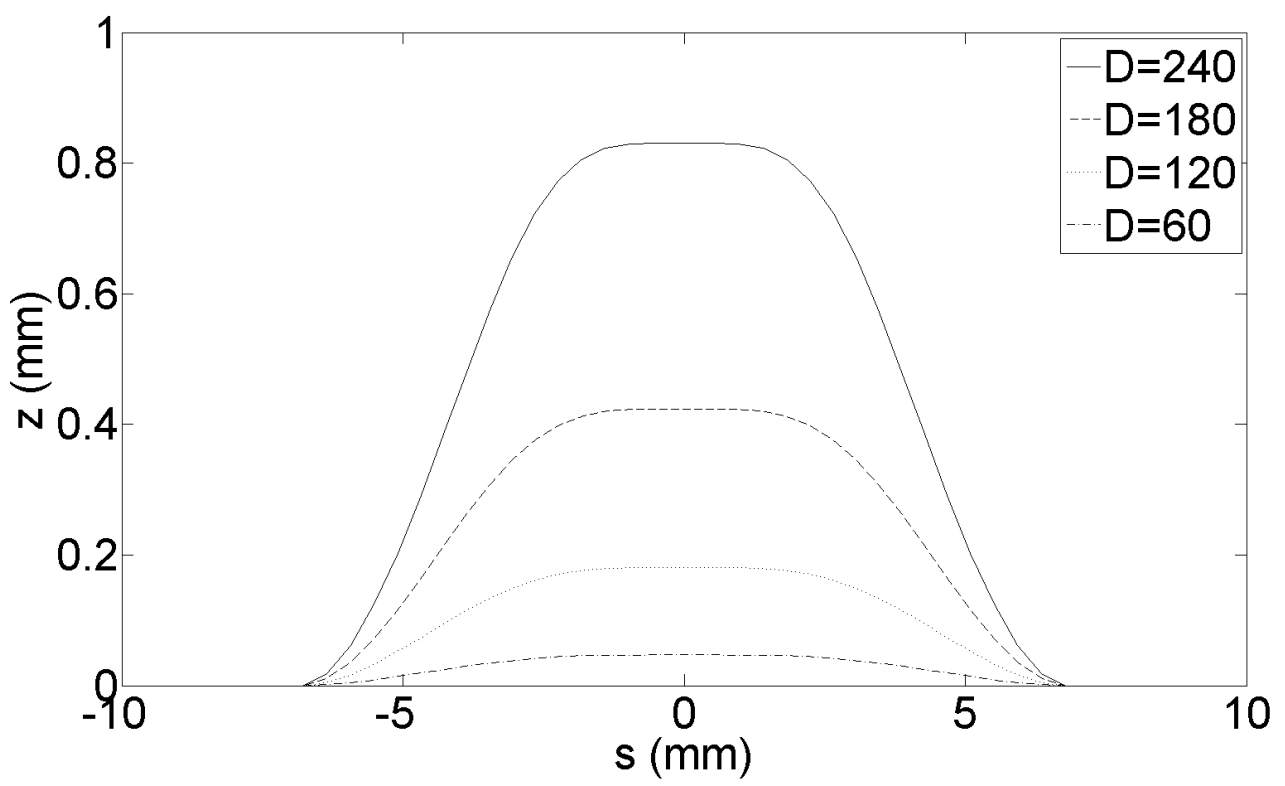

Figure 6.13: Equilibrium displacement of a square membrane for a cross section (at $u_{1}=u_{2}$ ) for a variety of dipole strengths with a pre-stretch of 1.2 , where the values of $D$ given in the figure are multiplied by $\times 10^{-6} \mathrm{Am}^{2}$.

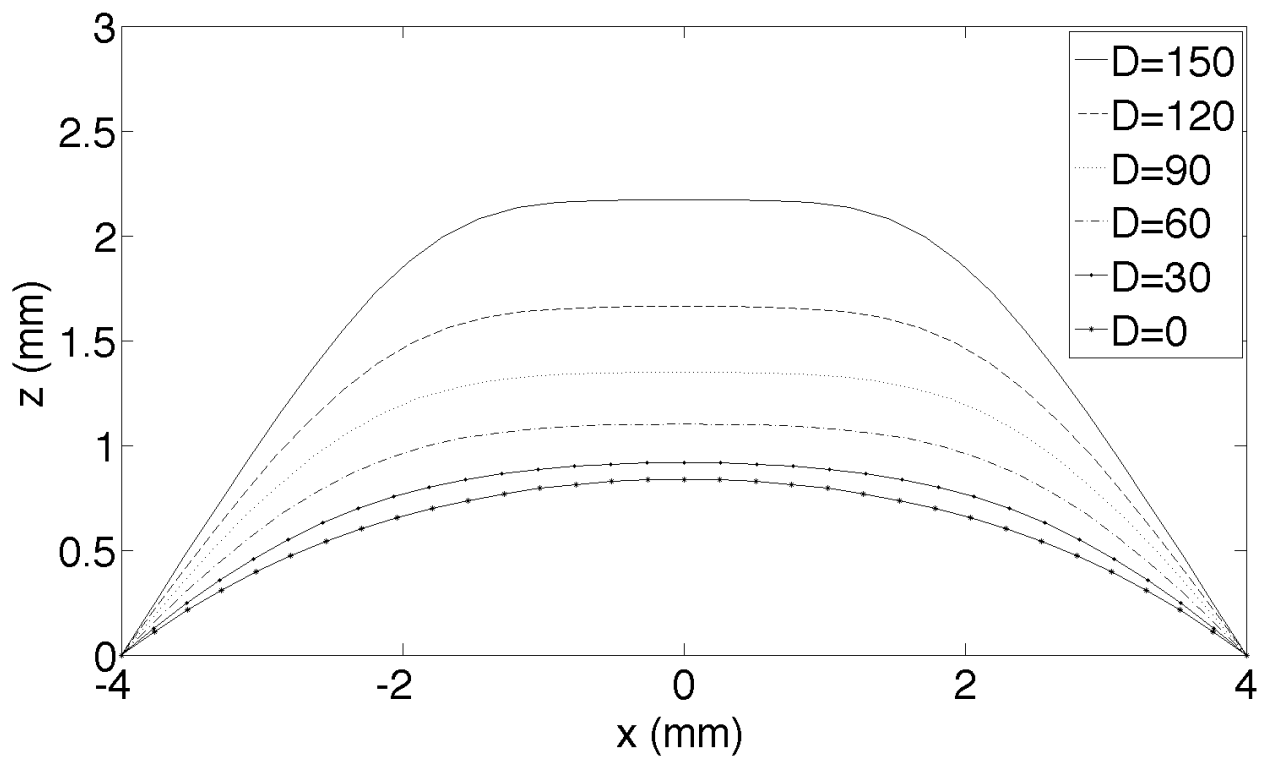

Figure 6.14: Equilibrium displacement of a square membrane for a cross section (at $\left.u_{2}=0\right)$ for a variety of dipole strengths subjected to a constant pressure, where the values of $D$ given in the figure are multiplied by $\times 10^{-6} \mathrm{Am}^{2}$. 


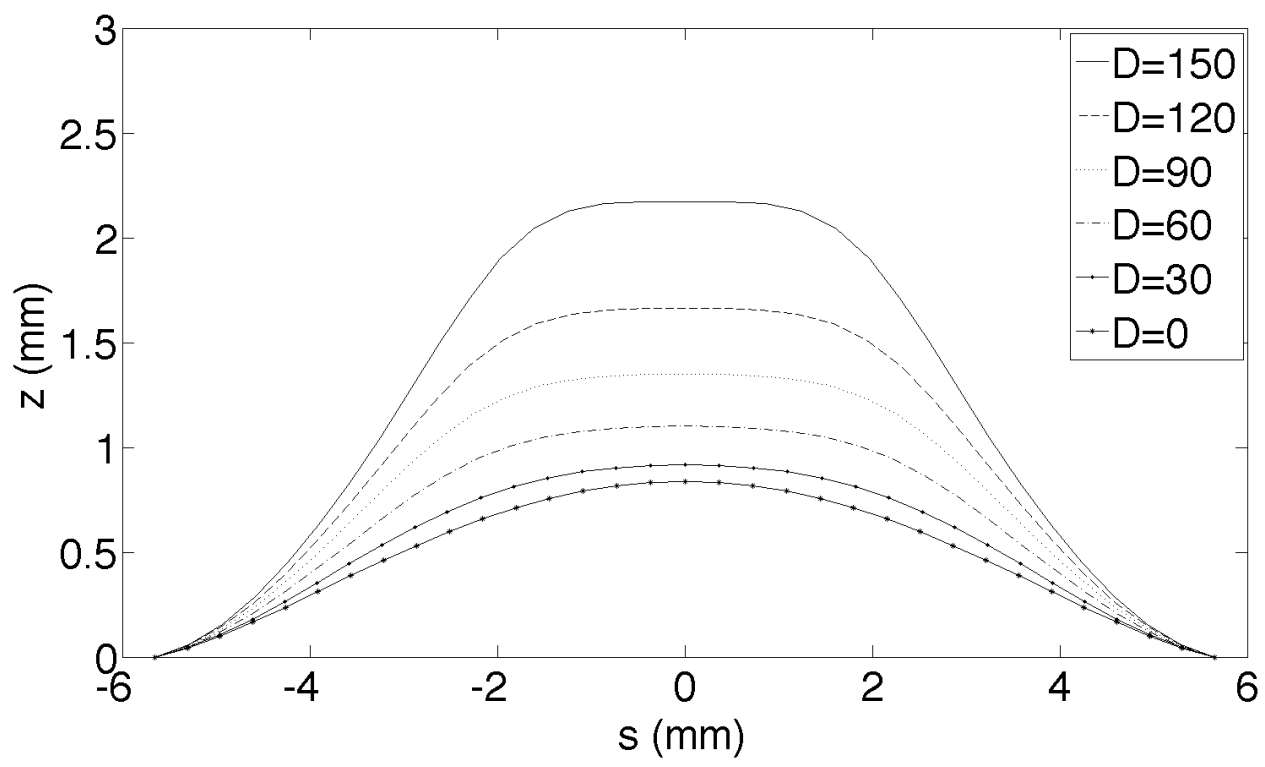

Figure 6.15: Equilibrium displacement of a square membrane for a cross section (at $u_{1}=u_{2}$ ) for a variety of dipole strengths subjected to a constant pressure, where the values of $D$ given in the figure are multiplied by $\times 10^{-6} \mathrm{Am}^{2}$.

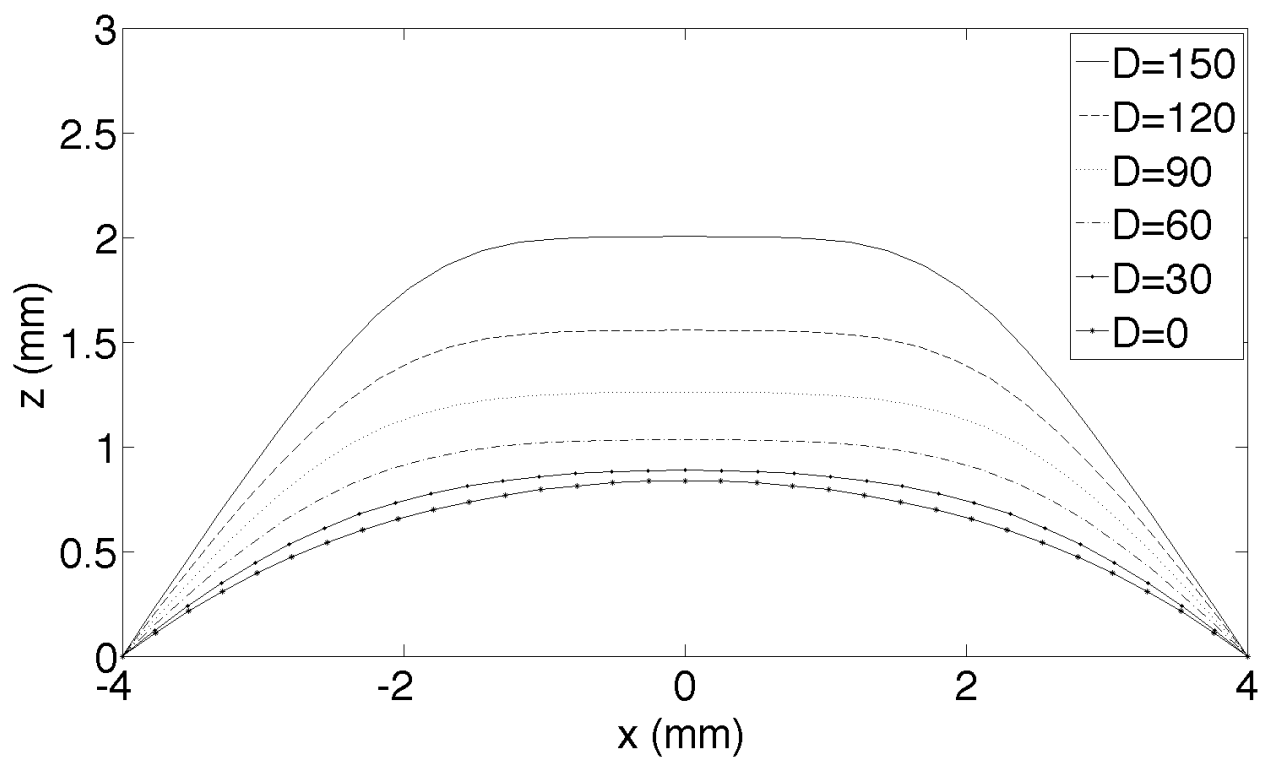

Figure 6.16: Equilibrium displacement of a square membrane for a cross section (at $u_{2}=0$ ) for a variety of dipole strengths with a pressure dependent on the volume, where the values of $D$ given in the figure are multiplied by $\times 10^{-6} \mathrm{Am}^{2}$. 


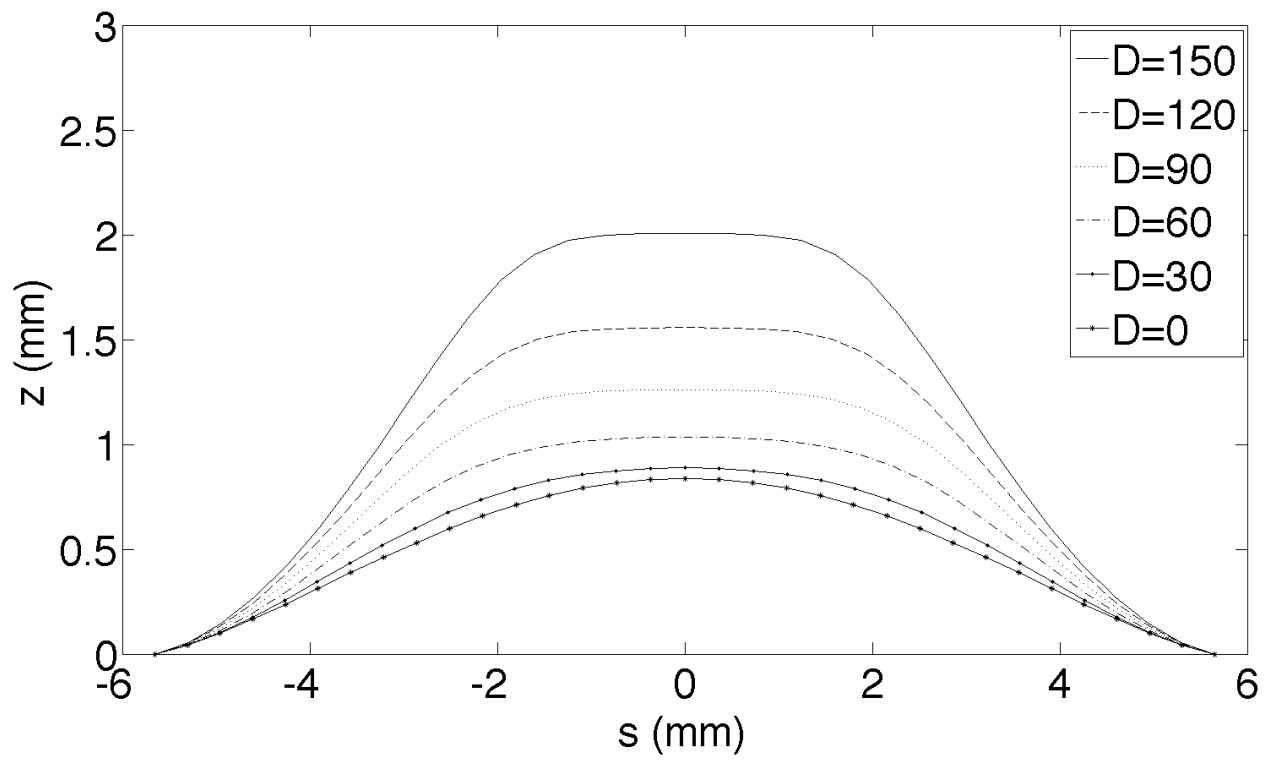

Figure 6.17: Equilibrium displacement of a square membrane for a cross section (at $\left.u_{1}=u_{2}\right)$ for a variety of dipole strengths with a pressure dependent on the volume, where the values of $D$ given in the figure are multiplied by $\times 10^{-6} A m^{2}$.

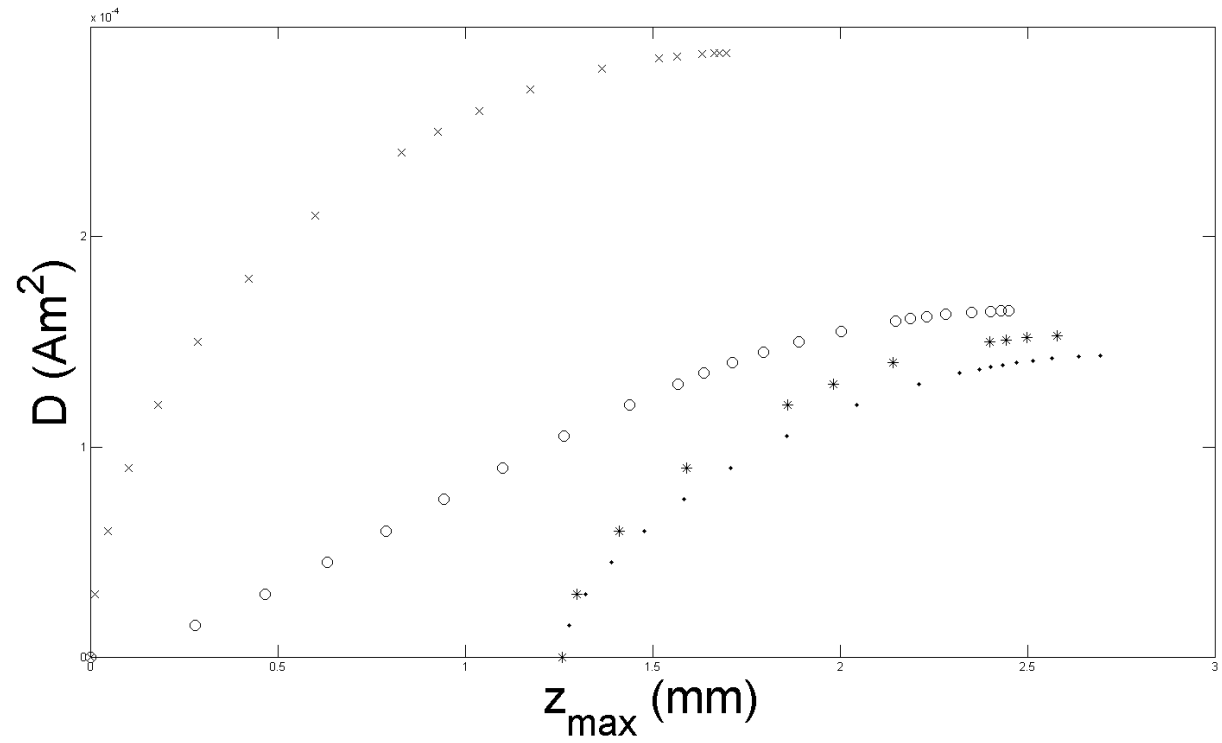

Figure 6.18: Membrane displacement under the dipole source, as a function of dipole strength. Effect of pre-stretch indicated by circles (o) and crosses $(\times)$; effect of fixed or volume dependent pressure is indicated by dots $(\cdot)$ and stars $(*)$, respectively (see text). 
much smaller deflection at any given field strength. The effect of pressure (at no pre-stretch) is illustrated by the dotted and starred data, the former corresponding to a fixed inflation pressure $P=6.0 \times 10^{5} \mathrm{~Pa}$ acting on the interior of the membrane; the external pressure is assumed to vanish. The stars correspond to a volume-dependent pressure. As expected, pressure has a significant effect on deformation at small field intensities, but its relative importance diminishes with increasing intensity. Moreover, at any value of field intensity the volume-dependent pressure yields a smaller displacement than that produced by the fixed pressure. The discrepancy increases with field intensity due to the attendant increase in volume, which causes the volume-dependent pressure to be reduced in magnitude. In all cases an upper limit is predicted for the deformation that can be maintained in equilibrium. Such limits are identified by the failure of the dynamic relaxation method to generate equilibria when the field intensity is increased above a critical value. Our results thus establish the existence of a limit-point instability at sufficiently high field intensities. This corroborates the analysis of Section 5.2, based on a low-order finite-dimensional projection of the model developed in Section 5.1. For an example simulation with a dipole that does not have an equilibrium deformation see Figure 6.19. and 6.20 It is seen that as time progresses the

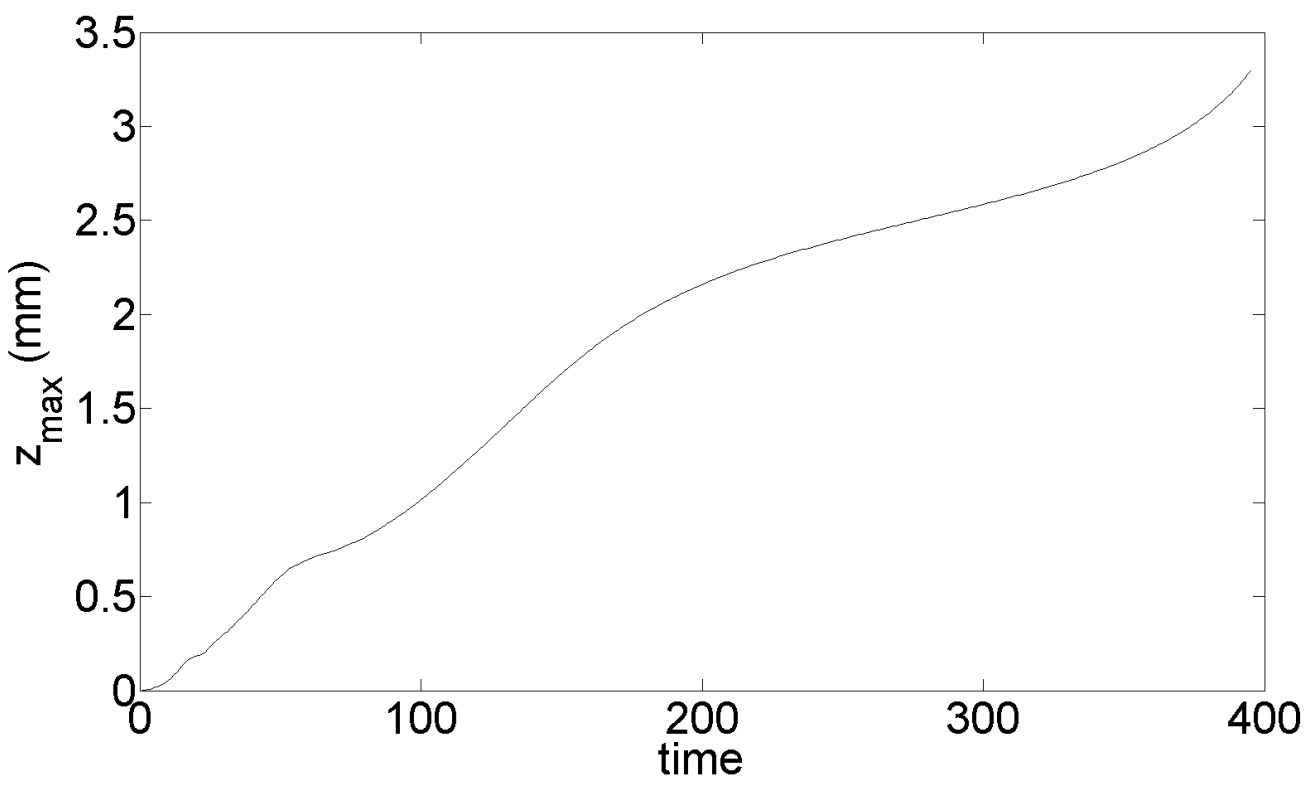

Figure 6.19: Maximum nodal $z$-displacement for $D=170 \times 10^{-6} A m^{2}$ versus time for square membrane.

speed starts to approach zero but at around time $=300$ it starts to increase rapidly until the simulation is stopped. For the maximum $z$ displacement it starts to approach a constant value but around time $=300$ it begins to rapidly increase. Together these results show that the simulation does not have a stable equilibrium. 


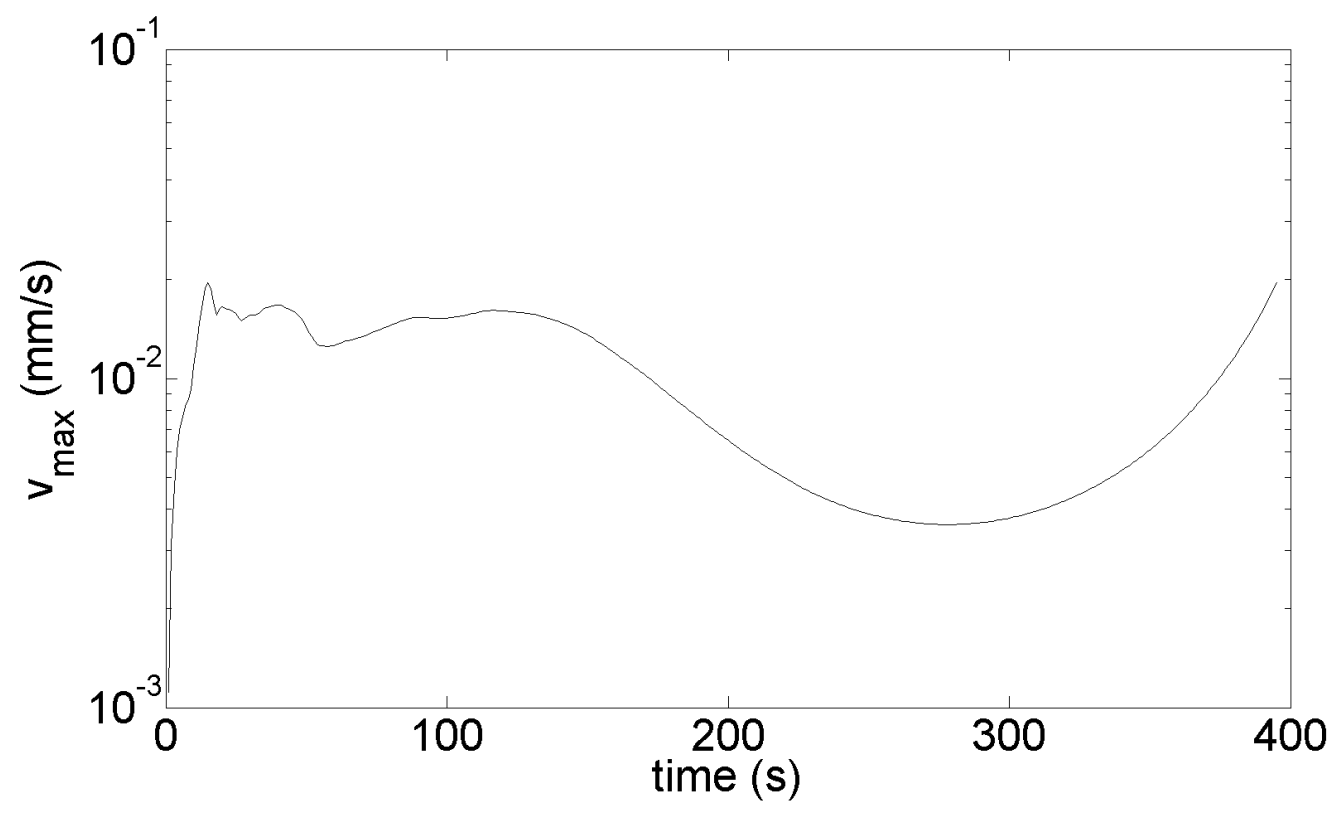

Figure 6.20: Maximum nodal velocity for $D=170 \times 10^{-6} A m^{2}$ versus time for square membrane.

So far we have only looked at membrane deformations where the dipole is placed above the center of the membrane. We now look at deformations where the dipole is moved over the edge of the membrane, in the $x$-direction. Figures 6.21 and 6.22 illustrates the same deformation at a dipole strength of $D=240 \times 10^{-6} A m^{2}$ with the shifted dipole source. A cross section for a dipole that has been shifted over the edge of the membrane is shown in Figure 6.23 for $u_{2}=0.0$. The ability to solve problems with a shifted dipole source is possible due to the robustness of the finite difference method. 


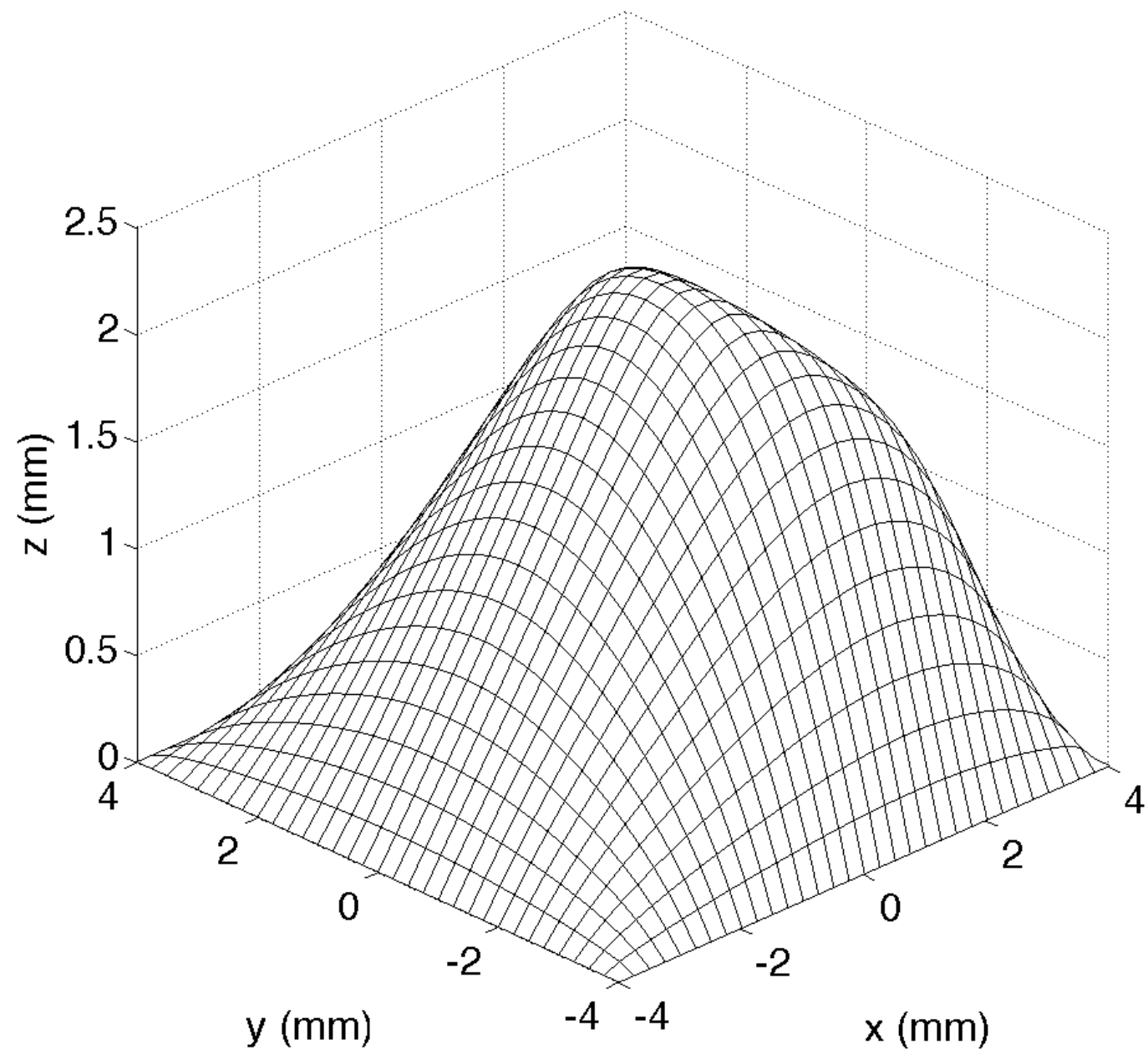

Figure 6.21: Deformed square membrane for $D=240 \times 10^{-6} \mathrm{Am}^{2}$ and dipole position of $\mathbf{y}_{d}=\{4.0,0.0,8.0\} \mathrm{mm}$. 


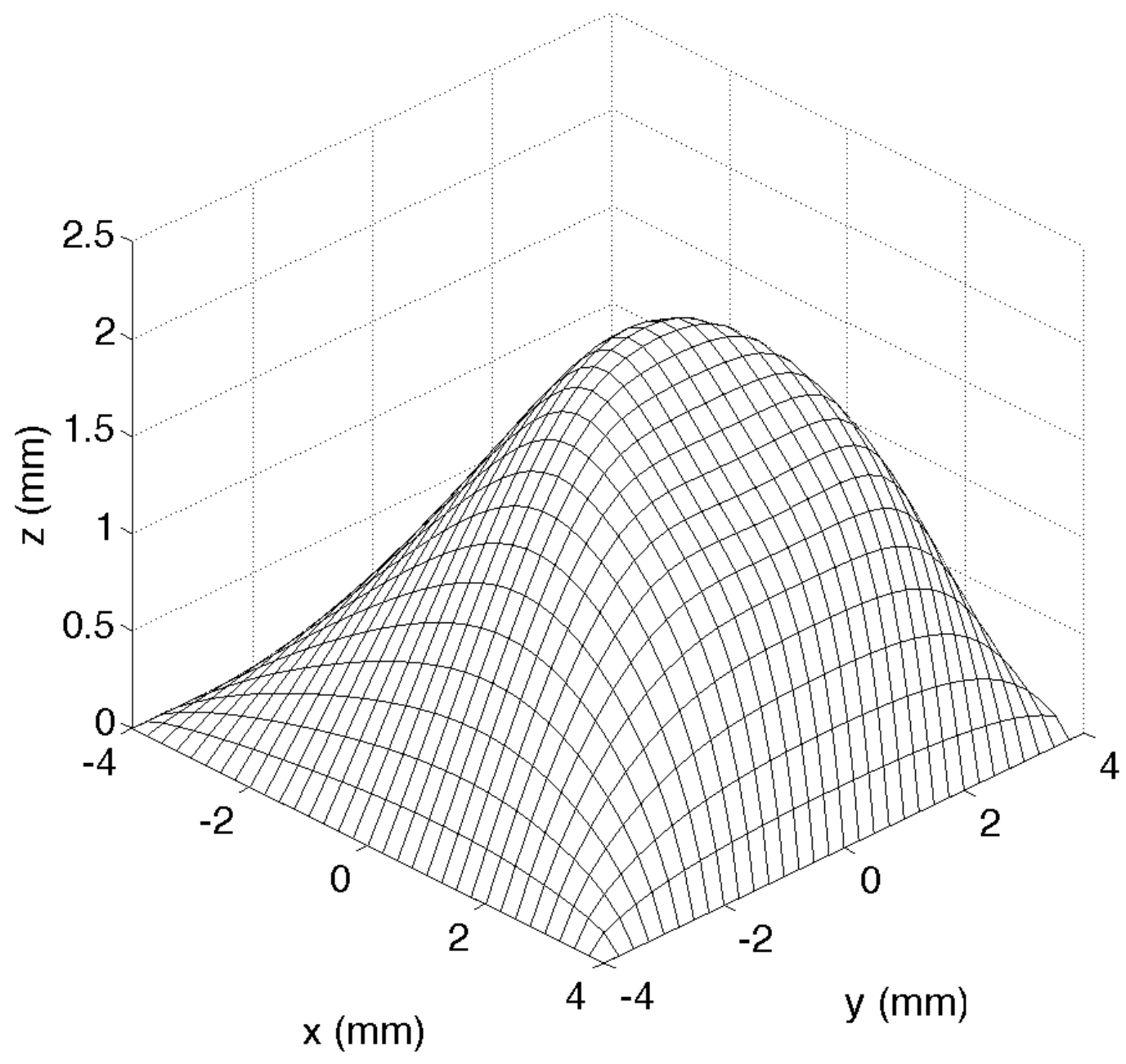

Figure 6.22: Deformed square membrane for $D=240 \times 10^{-6} \mathrm{Am}^{2}$ and dipole position of $\mathbf{y}_{d}=\{4.0,0.0,8.0\} \mathrm{mm}$. 


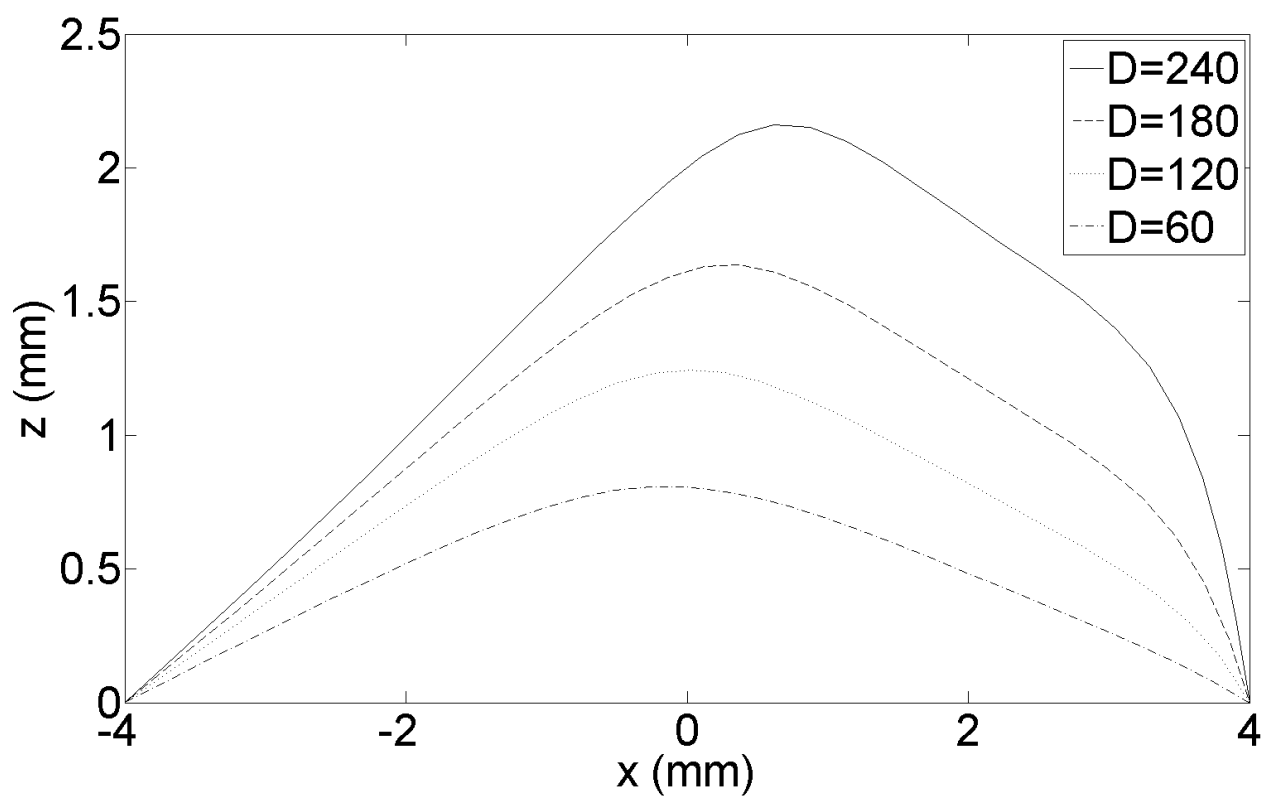

Figure 6.23: Equilibrium displacement of a circular membrane for a cross section (at $u_{2}=0$ ) for a variety of dipole strengths with a dipole position of $\mathbf{y}_{d}=\{4.0,0.0,8.0\} \mathrm{mm}$, where the values of $D$ given in the figure are multiplied by $\times 10^{-6} A m^{2}$. 


\subsubsection{Circular Geometry}

The second referential geometry we examine is circular, with a radius $4.0 \mathrm{~mm}$. In order to see if the number of nodes used in the simulations is large enough to capture the actual equilibrium deformation a refinement study was performed for a dipole of strength $D=200 \times 10^{-6} \mathrm{Am}^{2}$. The center displacement of the membranes is compared, see Figure 6.24. where the relation between the two "butterfly" mesh quantities $N_{r}=\left(N_{c}-1\right) / 4$ is

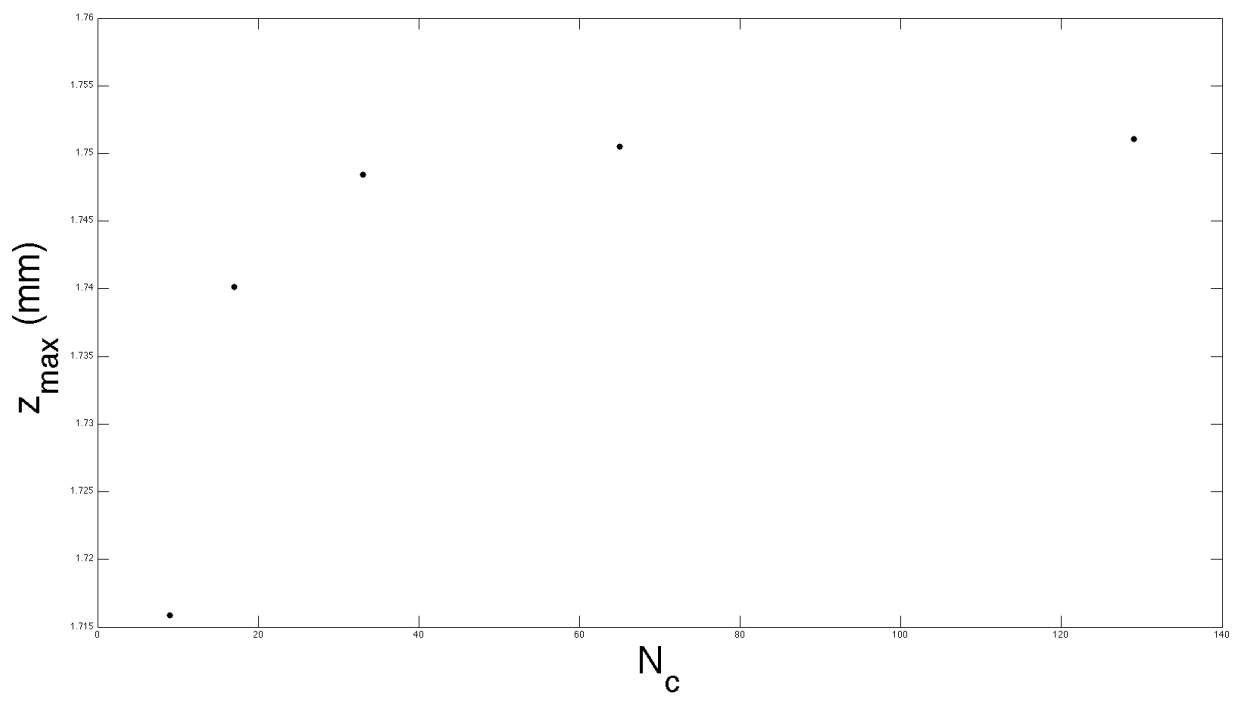

Figure 6.24: Circular membrane center displacement for a variety of meshes for $D=180 \times$ $10^{-6} \mathrm{Am}^{2}$ and zero pressure.

enforced. It is seen that as the number of nodes $\left(N_{c}\right)$ is increased the center displacement asymptotically approaches a constant value. It is seen that $N_{c}=17$ produces results with $0.6240 \%$ error when the most refined simulation $\left(N_{c}=129\right)$ is assumed to be the exact solution. All further simulations will be performed with $N_{c}=17$. Similar analysis for the circular membrane was preformed to check that the tol of equation 4.20 as was done for the square membrane.

Figure 6.25 depicts the deformation of the circular membrane under zero pressure in response to a dipole of strength $D=200 \times 10^{-6} A m^{2}$ (cf. 2.35). The vertical and in-plane dimensions are scaled differently to aid in visualization.

Again we have used the data generated by the simulation, together with 3.13 , to verify that the three-dimensional principal stretches on the membrane surface are well within the limits required for the validity of $2.116_{2}$. The referential in-plane magnetization field $\mathbf{M}$ is shown in Figure 6.26. This field is consistently directed toward the center of the membrane, where differentiability requires that its intensity diminish to zero. This and the constraint 


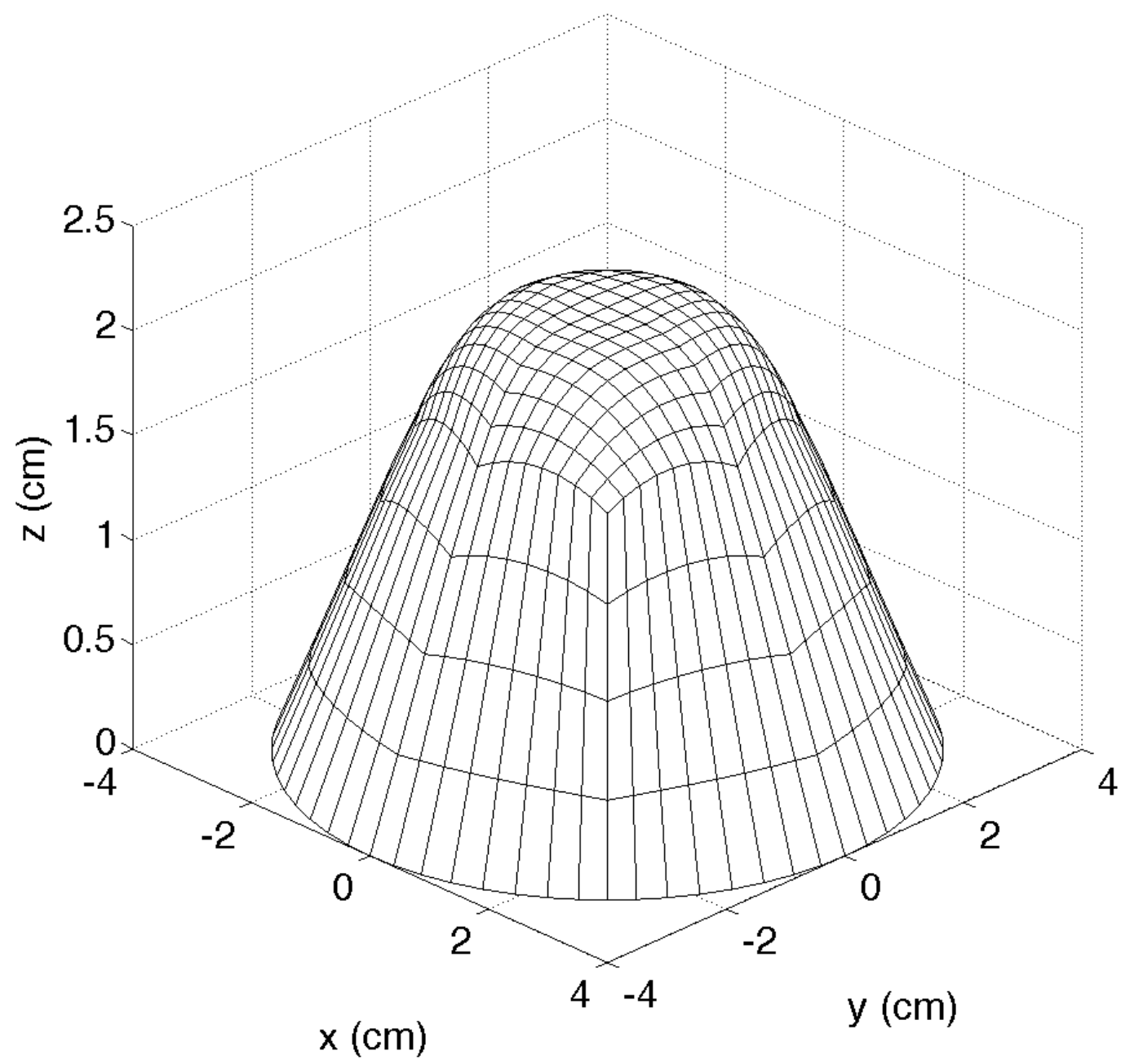

Figure 6.25: Deformed circular membrane for $D=200 \times 10^{-6} \mathrm{Am}^{2}$. 


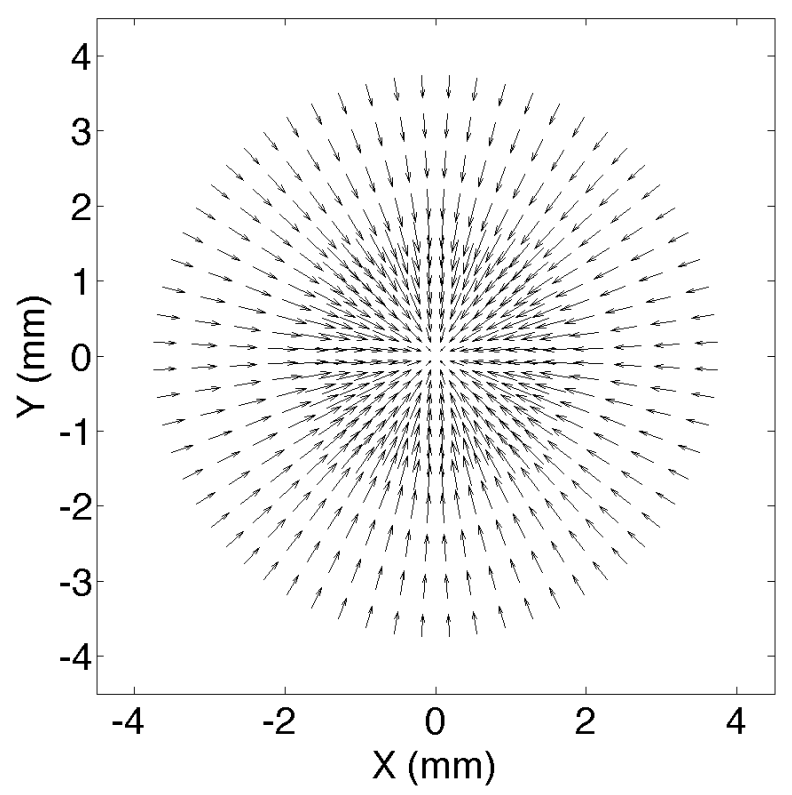

Figure 6.26: Reference magnetization (M) for $D=200 \times 10^{-6} A m^{2}$ on the reference circular membrane.

3.35 cause the interaction with the applied field to weaken near the center, resulting in a deformed surface that is relatively flat under the dipole source.

Figure 6.27 shows the variation of the in-plane part, e, of the director field with respect to position on the reference plane. The deformation deviates from Kirchhoff-Love kinematics wherever this is nonzero. This reflects the bias induced by the dipole source at points lying off the dipole axis, causing the director $\mathbf{d}$ on the deformed surface to tilt relative to the tangent plane as the membrane adjusts to the applied field. The effect diminishes near the edge of the membrane where the field is relatively weak, and near the center where the field lines intersect the membrane orthogonally and the associated bias vanishes; in either case the kinematics revert to the Kirchhoff-Love mode.

Figure 6.28 shows the current magnetization $(\mathbf{m})$ on the deformed membrane where 2.104 is used to push the referential magnetization forward.

In order to see how the deformation changes at different dipole strength we look at cross sections of the displacement for a variety of dipole strengths. Figure 6.29 shows the cross section deformation at $u_{2}=0.0$.

Next the effect of uniform pre-stretch of 1.1 induced by an outward displacement of nodes on the boundary; these are subsequently fixed in the course of the simulation. Cross sections of the displacement with pre-stretch for a variety of dipole strengths are shown in Figure 6.30 , which shows the cross section deformation at $u_{2}=0.0$.

The equilibrium deformation effect of pressure (at no pre-stretch) at a fixed inflation 


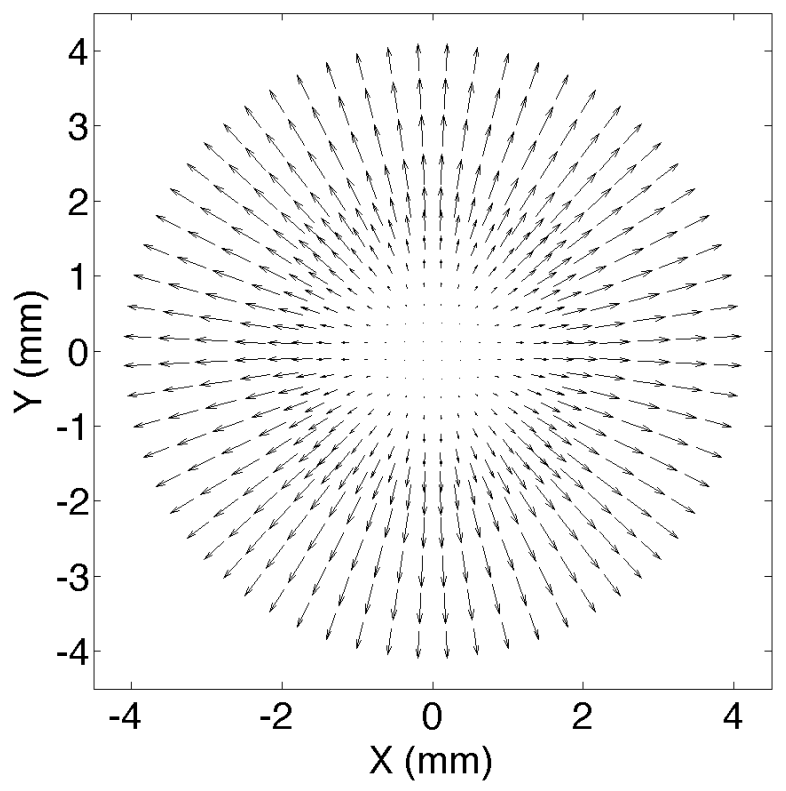

Figure 6.27: Unknown portion of the director (e) for $D=160 \times 10^{-6} \mathrm{Am}^{2}$ on the reference circular membrane.

pressure $P=2.0 \times 10^{5} \mathrm{~Pa}$ acting on the interior of the membrane; the external pressure is assumed to vanish. This is regarded as being supplied by a large reservoir with an opening on the reference plane. Cross sectional deformations are illustrated in Figure 6.31 showing the cross section deformation at $u_{2}=0.0$.

Next we examine a volume-dependent pressure in which the product of the pressure and the enclosed volume remains constant. The constant is derived by using 3.74 to compute the contained volume generated in response to the fixed pressure at zero field strength. The new pressure is again determined by 6.3 . Cross sections of the deformation are illustrated in Figure 6.32 and show the cross section deformation at $u_{2}=0.0$.

Finally, the effects of pre-stretch and pressure are compared in Figure 6.33, in which the height of the deformed surface, at a point on the dipole axis, is plotted against dipole strength. The open circles and crosses correspond to zero applied pressure; the former corresponding to no pre-stretch and the latter to a uniform pre-stretch of 1.1 induced by an outward displacement of nodes on the boundary; these are subsequently fixed in the course of the simulation. Pre-stretch is seen to stiffen the membrane dramatically, resulting in a much smaller deflection at any given field strength. The effect of pressure (at no pre-stretch) is illustrated by the dotted and starred data, the former corresponding to a fixed inflation pressure $P=2.0 \times 10^{5} \mathrm{~Pa}$ acting on the interior of the membrane; the external pressure is assumed to vanish. The stars correspond to a volume-dependent pressure in which the product of the pressure and the enclosed volume remains constant, as in an ideal gas at 


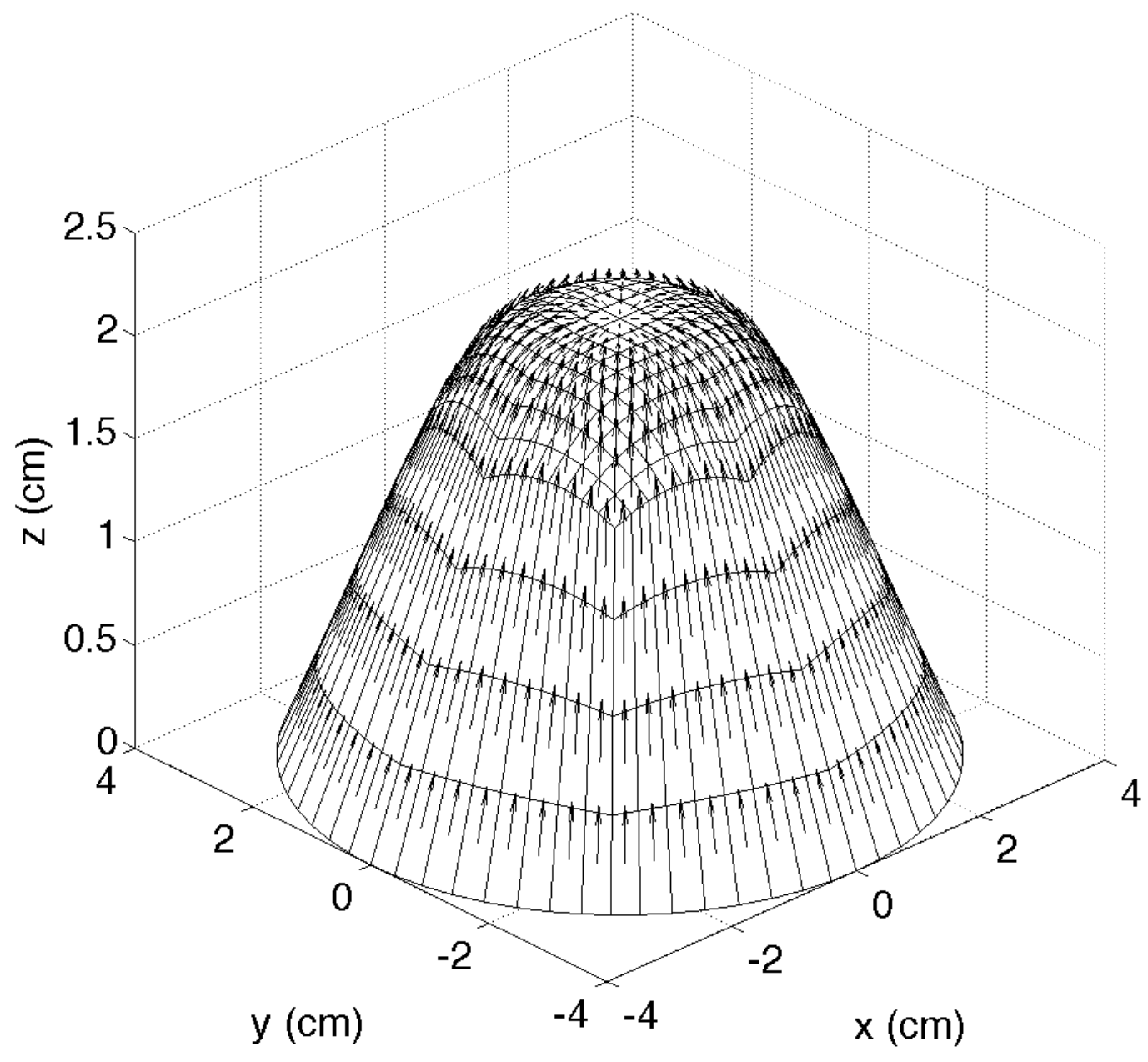

Figure 6.28: Current magnetization $(\mathbf{m})$ for $D=200 \times 10^{-6} A m^{2}$ on the deformed circular membrane. 


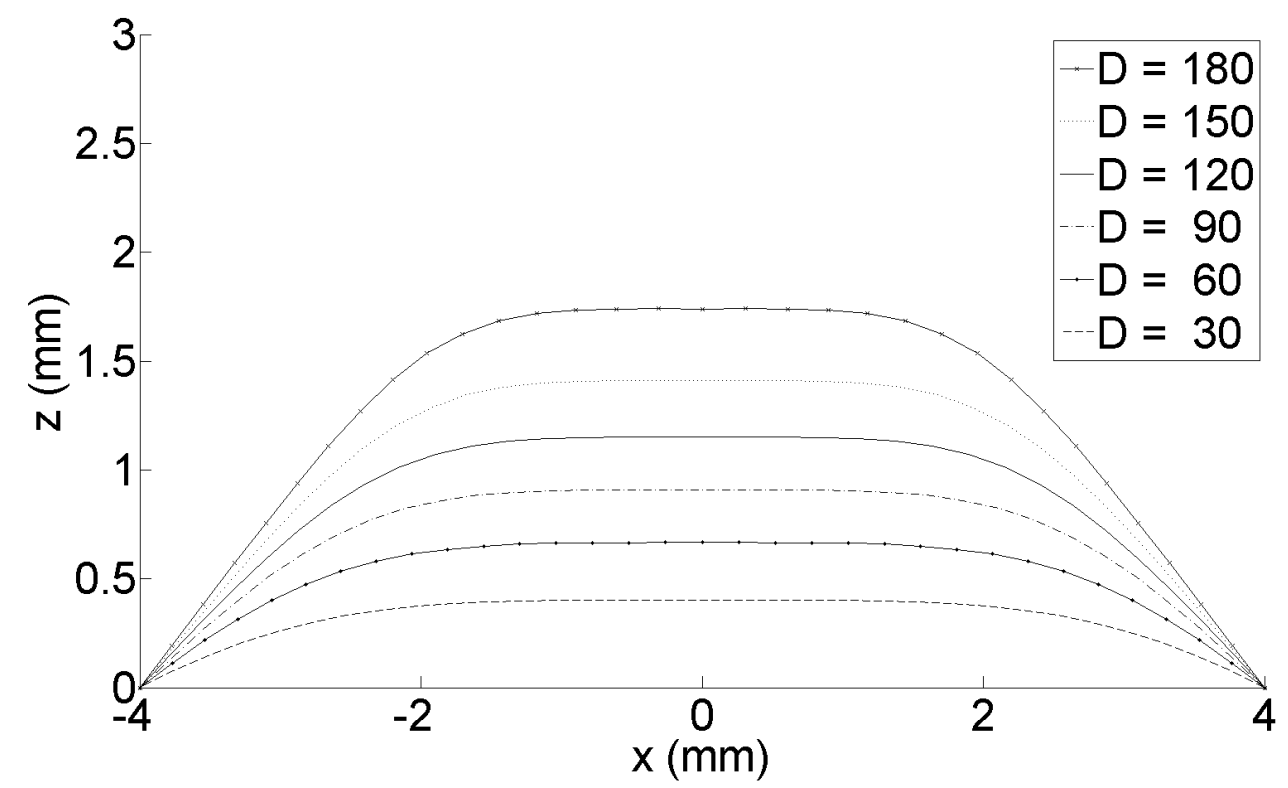

Figure 6.29: Equilibrium displacement of a circular membrane for a cross section (at $u_{2}=0$ ) for a variety of dipole strengths, where the values of $D$ given in the figure are multiplied by $\times 10^{-6} \mathrm{Am}^{2}$.

fixed temperature. As expected, pressure has a significant effect on deformation at small field intensities, but its relative importance diminishes with increasing intensity. Moreover, at any value of field intensity the volume-dependent pressure yields a smaller displacement than that produced by the fixed pressure. The discrepancy increases with field intensity due to the attendant increase in volume, which causes the volume-dependent pressure to be reduced in magnitude. In all cases an upper limit is predicted for the deformation that can be maintained in equilibrium. Such limits are identified by the failure of the dynamic relaxation method to generate equilibria when the field intensity is increased above a critical value. Our results thus establish the existence of a limit-point instability at sufficiently high field intensities. This corroborates the analysis of 5.2, based on a low-order finite-dimensional projection of the model developed in Section 5.1.

We next examine deformations of the circular membrane where the dipole is moved, in the $x$-direction, over the edge of the membrane. Figures 6.34 and 6.35 illustrate the same deformation at a dipole strength of $D=300 \times 10^{-6} \mathrm{Am}^{2}$ with the shifted dipole source.

A cross section for a dipole that has been shifted over the edge of the membrane are shown in Figure 6.36 for $u_{2}=0.0$. 


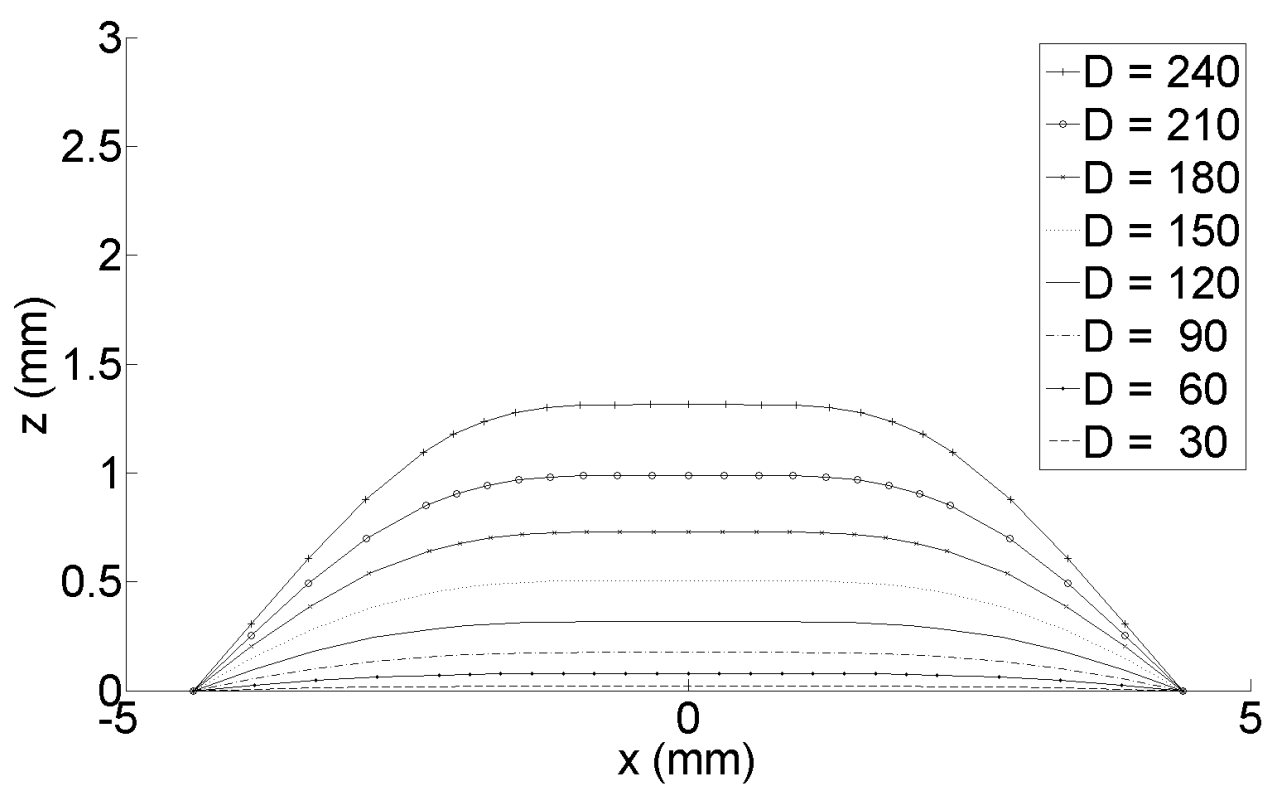

Figure 6.30: Equilibrium displacement of a circular membrane for a cross section (at $u_{2}=0$ ) for a variety of dipole strengths with pre-stretch of 1.1 , where the values of $D$ given in the figure are multiplied by $\times 10^{-6} \mathrm{Am}^{2}$. 


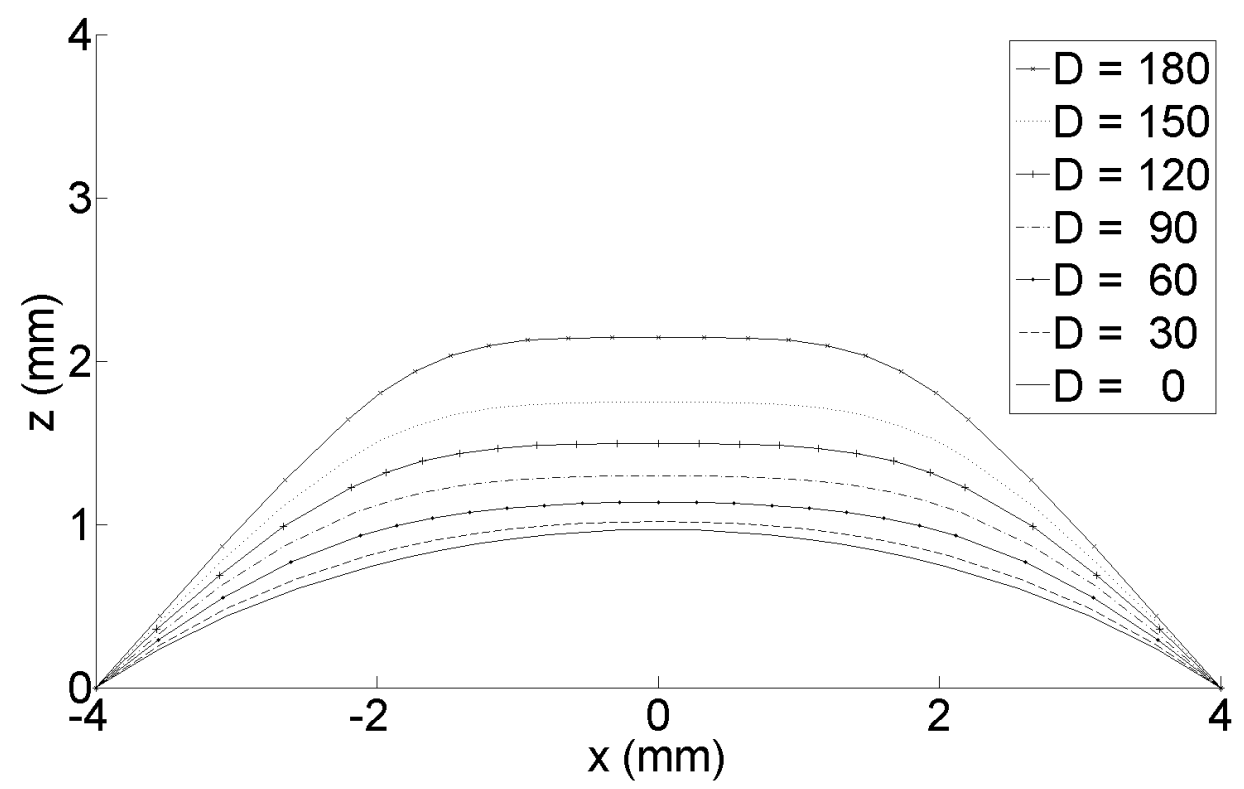

Figure 6.31: Equilibrium displacement of a circular membrane for a cross section $\left(\right.$ at $\left.u_{2}=0\right)$ for a variety of dipole strengths subjected to a constant pressure, where the values of $D$ given in the figure are multiplied by $\times 10^{-6} \mathrm{Am}^{2}$. 


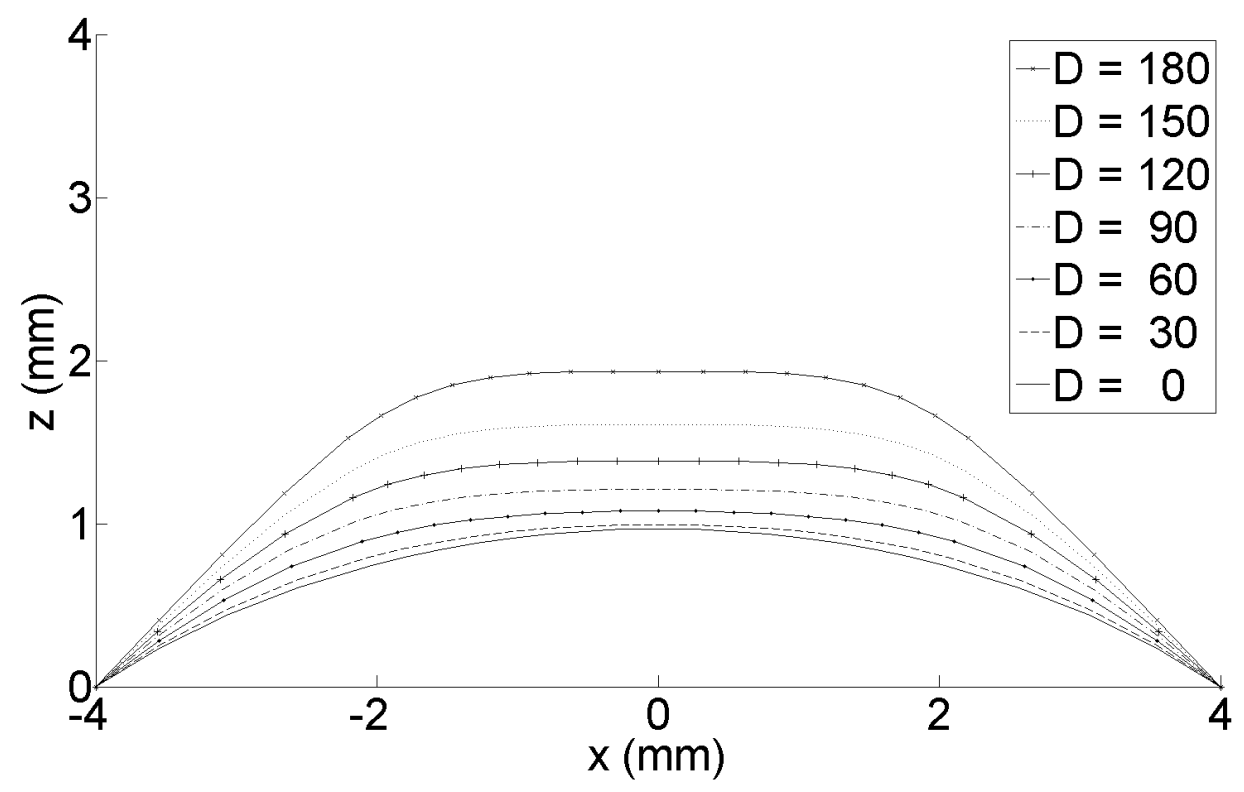

Figure 6.32: Equilibrium displacement of a circular membrane for a cross section (at $u_{2}=0$ ) for a variety of dipole strengths with a pressure dependent on the volume, where the values of $D$ given in the figure are multiplied by $\times 10^{-6} \mathrm{Am}^{2}$.

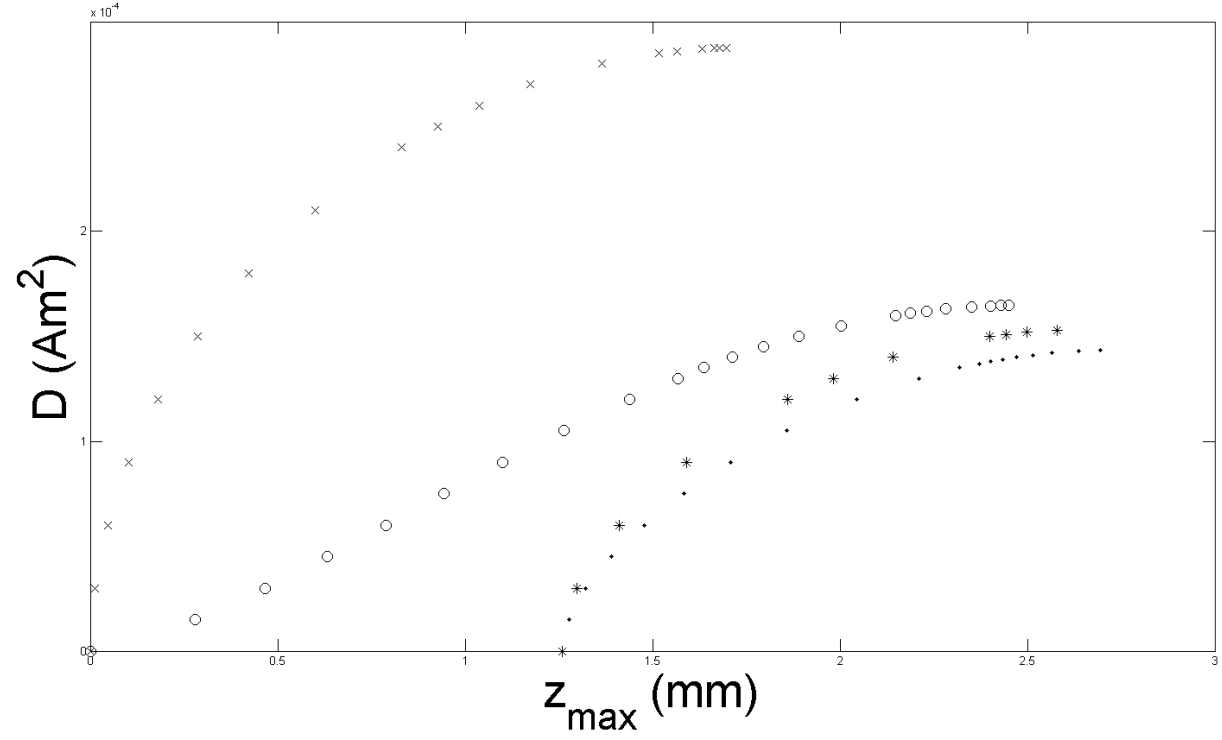

Figure 6.33: Comparison of a circular membrane maximum z-displacement. 


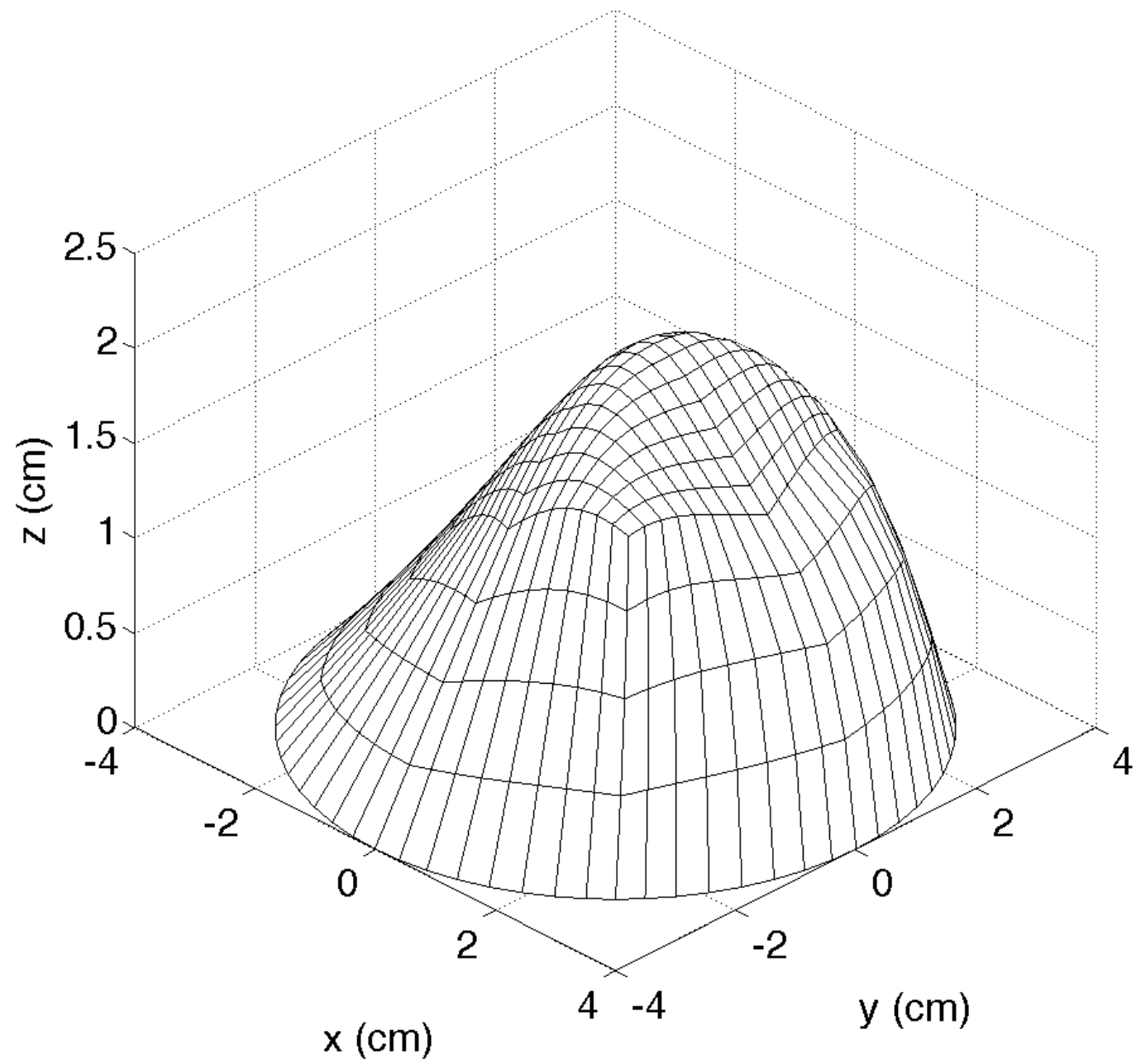

Figure 6.34: Deformed circular membrane for $D=300 \times 10^{-6} \mathrm{Am}^{2}$ and dipole position of $\mathbf{y}_{d}=\{4.0,0.0,8.0\} \mathrm{mm}$. 


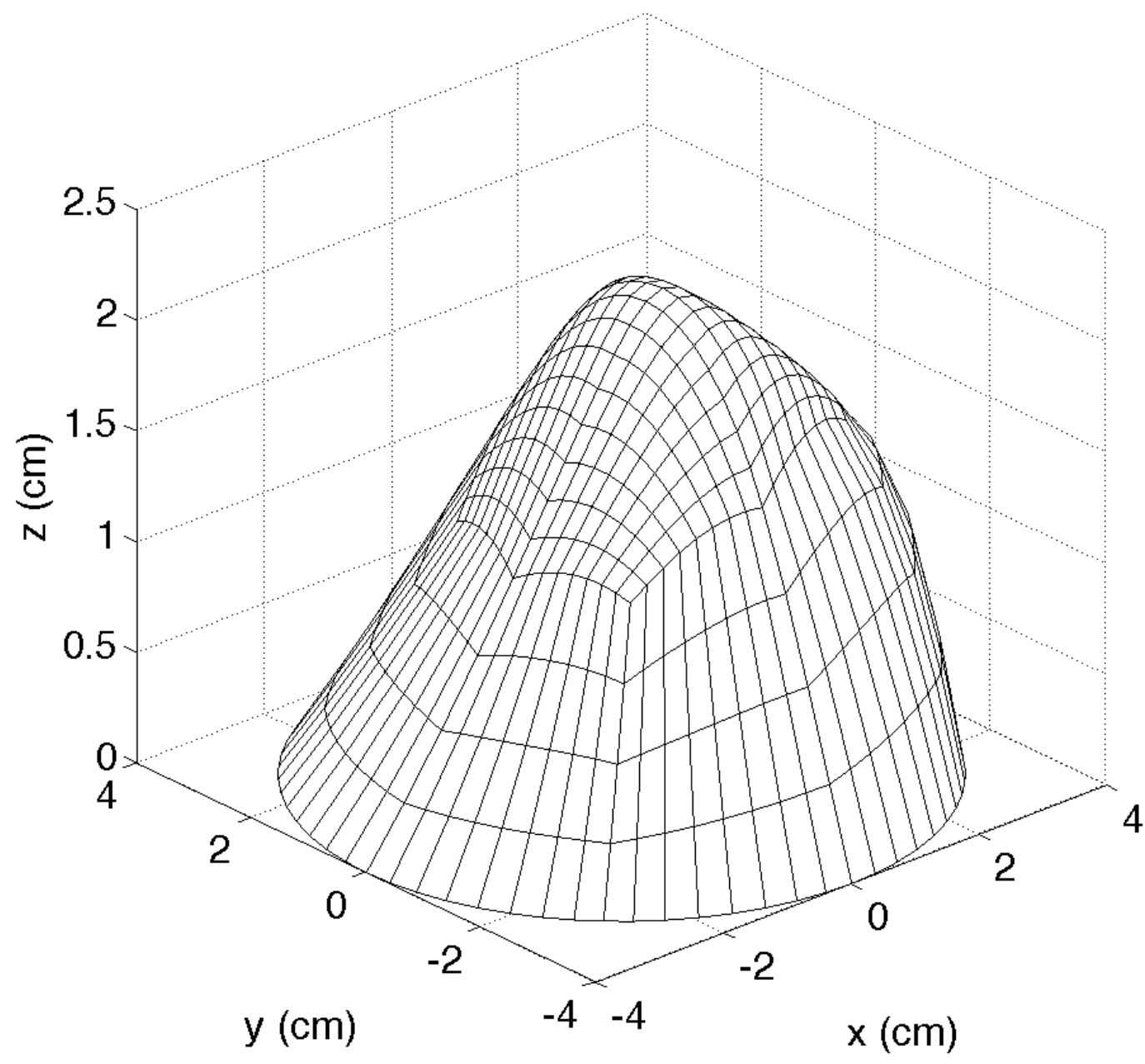

Figure 6.35: Deformed circular membrane for $D=300 \times 10^{-6} \mathrm{Am}^{2}$ and dipole position of $\mathbf{y}_{d}=\{4.0,0.0,8.0\} \mathrm{mm}$. 


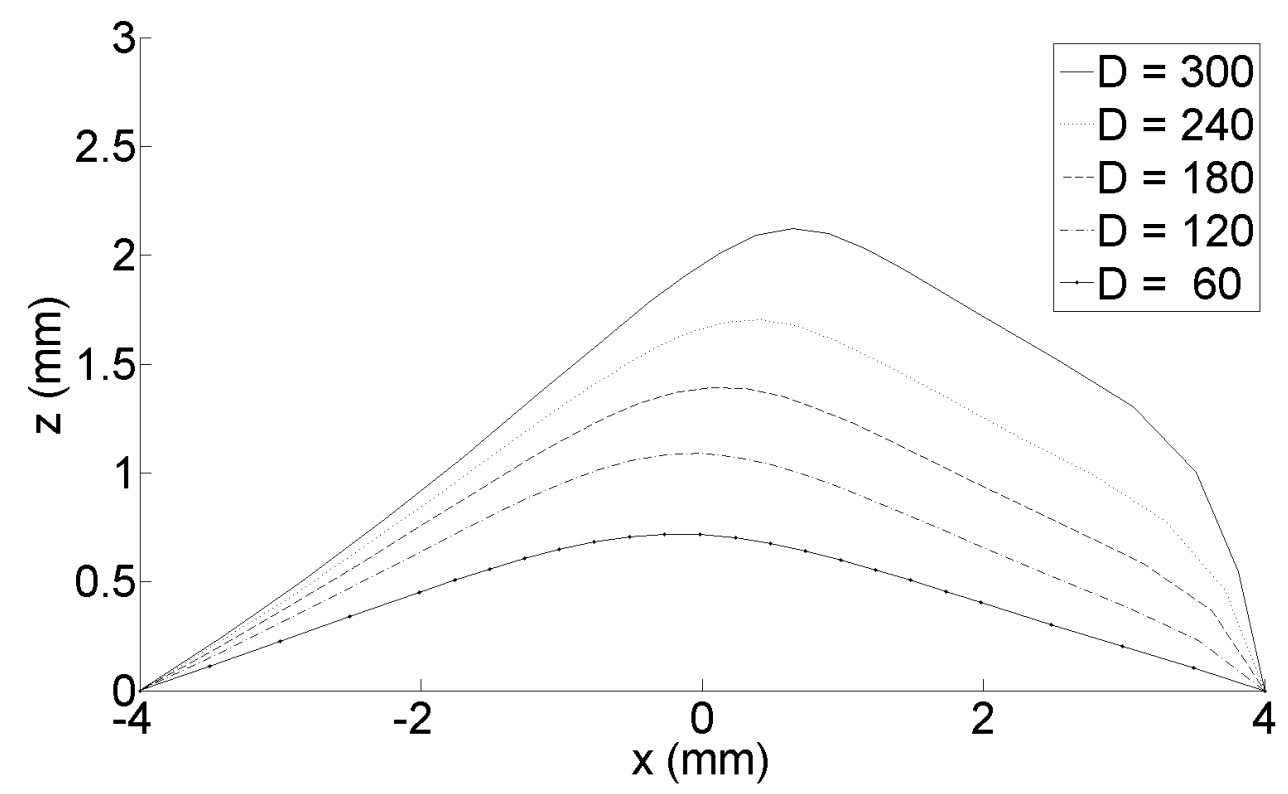

Figure 6.36: Equilibrium displacement of a circular membrane for a cross section (at $y=0)$ for a variety of dipole strengths with a dipole position of $\mathbf{y}_{d}=\{4.0,0.0,8.0\} \mathrm{mm}$, where the values of $D$ given in the figure are multiplied by $\times 10^{-6} \mathrm{Am}^{2}$. 


\section{Chapter 7}

\section{Conclusion}

We have developed an approximate membrane theory from three-dimensional magnetoelasticity for magnetizable materials that are highly deformable, synthesized from elastomers infused with micro- or nano-scopic ferrous particles [19]. This approach is more systematic than alternative formulations based, for example, on thickness-wise integration of the local three-dimensional balance laws (e.g. [12]). It incorporates a constraint requiring the magnetization to remain tangential to the film as it deforms. This was motivated by the fact such states are energetically optimal in thin films [11] and [34]. Likewise, we imposed the constraint of bulk incompressibility, and thus excluded dilatational modes of deformation that are energetically unfavorable in typical elastomers. However, unlike incompressibility, the constraint on magnetization is not of the kind that requires a reactive Lagrange multiplier in the relevant constitutive equation. Rather, it is a restriction involving the deformation, allowing local membrane geometry to adjust in response to an applied field. The Kirchhoff-Love type constraints is not imposed at the outset as in many other theories of thin magnetoelastic plates [41]. In general such constraints impede the attainment of minima of the overall energy because, by confining our attention to states of magnetization that are optimal at any deformation, we have effectively eliminated magnetization as an independent variable. The bias induced by an applied field yields deformations that violate constraints of the Kirchhoff-Love type. Here, this was addressed via a director field which emerges naturally from the underlying three-dimensional theory in the manner described in [36] for the purely mechanical problem, without restricting the nature of the deformation in thin bodies.

We use a finite-difference method to discretize the model spatially and discuss the solution of the resulting equations by the method of dynamic relaxation, in which equilibria are obtained as long-time limits of solutions to an artificial dynamical system with viscosity. This finite-difference method was expanded to better handle circular films, by the introduction of a "butterfly" mesh.

This model was then applied to simulate the interplay between an applied magnetic field, modeled here as a remote dipole source, and the pressure transmitted to the membrane by 
a confined gas for the two types of material models.

First the weak material model was examined and we solved for the equilibrium deformation using a shooting method (where we further simplify the problem to axisymmetric deformations), finite element method and the finite-difference method. An instability was inferred from the deformation response and then further investigated with a variational method.

Second, the finite-difference method was applied to determine the deformation, magnetization and magnetic field generated by a thin film, using the Kankanala \& Triantafyllidis material model, in response to an applied magnetic field, pre-stretch, and pressure load. Here it is seen that the The Kirchhoff-Love type constraints is violated. This is due to the fact that, in genera case, magnetization and deformation are coupled in the expression for the magnetoelastic strain-energy function. 


\section{Bibliography}

[1] A. Atai and D.J. Steigmann. Coupled deformations of elastic curves and surfaces. Int. J. Solids \& Structures, 35:1915-1952, 1998.

[2] M. Barham, D. J. Steigmann, M. McElfresh, and R. E. Rudd. Finite deformation of a pressurized magnetoelastic membrane in a stationary dipole field. Acta Mech., 191:1-19, 2007.

[3] M. Barham, D. J. Steigmann, M. McElfresh, and R. E. Rudd. Limit-point instability of a magnetoelastic membrane in a stationary magnetic field. Smart Mater. Struct., $17: 1-6,2008$.

[4] M. Barham, D. White, D. J. Steigmann, and R. E. Rudd. Finite-element modeling of the deformation of a thin magnetoelastic film compared to a membrane model. IEEE Transactions on Magnetics, 45(10):4124-4127, 2009.

[5] M. Barham, D. White, and D.J. Steigmann. Finite-element modeling of the deformation of magnetoelastic film. J. Computational Physics, 229:6193-6207, 2010.

[6] W. F. Brown. Magnetoelastic Interactions. Springer, Berlin, 1966.

[7] R. Bustamante, A. Dorfmann, and R.W. Ogden. On variational formulations in nonlinear magnetoelastostatics. Math. Mech. Solids, 13:725-745, 2008.

[8] R. Bustamante, A. Dorfmann, and R.W. Ogden. Numerical solution of finite geometry boundary-value problems in nonlinear magnetoelasticity. Int. J. Solids 85 Structures, 48:847-883, 2011.

[9] A. Dorfmann and R.W. Ogden. Magnetoelastic modeling of elastomers. Euro. J. Mech. A/Solids, 22:497-507, 2003.

[10] A. Dorfmann and R.W. Ogden. Nonlinear magnetoelastic deformations of elastomers. Acta Mechanica, 167:13-28, 2004.

[11] G. Gioia and R.D. James. Micromagnetics of very thin films. Proc. R. Soc. London A, 453:213, 1997. 
[12] A. E. Green and P. M. Naghdi. On electomagnetc effects in the theroy of shells and plates. Phil. Trans. R. Soc. Lond. A, 309:559, 1983.

[13] E. M. Haseganu and D. J. Steigmann. Analysis of partly wrinkled membranes by the method of dynamic relaxation. Computational Mechanics, 14:596-614, 1994.

[14] E. M. Haseganu and D. J. Steigmann. Equilibrium analysis of finitely deformed elastic networks. Computational Mechanics, 17:359-373, 1996.

[15] W. Hermann and L.D. Bertholf. Computational Methods for Transient Analysis, chapter Explicit Lagrangian finite-difference methods, pages 361-416. Elsevier, Amsterdam, 1983.

[16] T. J. R. Hughes. The Finite Element Method: Linear Static and Dynamic Finite Elemet Analysis. Dover, New York, 1987.

[17] J. D. Jackson. Classical Electrodynamics. Wiley, 1962 (1st ed), 1975 (2nd ed).

[18] R. D. James. Configurational forces in magnetism with application to the dynamics of a smallscale ferromagnetic shape memory cantilever. Continuum Mechanics and Thermodynamics, 14:55-86, 2002.

[19] M.R. Jolly, J.D. Carlson, and B.C. Mu noz. A model of the behaviour of magnetorheological materials. Smart Mater. Struct., 5:607-614, 1996.

[20] S. V. Kankanala and N. Triantafyllidis. On finitely strained magnetorheological elastomers. Journal of the Mechanics and Physics of Solids, 52:2869-2908, 2004.

[21] S. V. Kankanala and N. Triantafyllidis. Magnetoelastic buckling of a rectangular block in plane strain. Journal of the Mechanics and Physics of Solids, 56:1147-1169, 2008.

[22] R.J. Knops and E.W. Wilkes. Handbuch der Physik, volume IVa/3, chapter Theory of elastic stability. Springer, Berlin, 1973.

[23] A. Kovetz. Electromagnetic Theory. University Press, Oxford, 200.

[24] L. D. Landau and E. M. Lifshitz. Electrodynamics of continuous media. ButtherworthHeinemann, Oxford, 2nd edition, 1996.

[25] P. M. Naghdi. Theory of shells and plates. Handbycg der Physuk (Flügge, S. ed.), VIa/2:425-640, 1972.

[26] W. Noll. The foundations of classical mechanics in light of recent advances in continuum mechanics. In The Axiomatic Method, with Spacial Reference to Geometry and Physics, Berkeley Symposium, pages 266-281, Amsterdam, 1959. North-Holland. 
[27] R.W. Ogden. Waves in isotropic elastic materials of hadamard, green or harmonic type. J. Mech. Phys. Solids, 18:149-163, 1970.

[28] R.W. Ogden. Non-linear Elastic Deformations. Dover, New York, 1997.

[29] A.C. Pipkin. The relaxed energy density for isotropic elastic membranes. IMA J. Appl. Math., 36:85, 1986.

[30] R. Rieben and D. White. Verification of high-order mixed finite element solution of transient magnetic diffusion problems. IEEE Trans. Mag., 42(1):25-39, 2006.

[31] R. N. Rieben, D. A. White, B. K. Wallin, and J. M. Solberg. On the continuum theory of deformable ferromagnetic solids. J. Comput. Phys., 226:534-570, 2007.

[32] S. A. Silling. Finite difference modeling of phase changes and localisation in elasticity. Comuter Methods in Applied Mechanics and Engineering, 70:251-273, 1988.

[33] S. A. Silling. Phase changes induced by deformation in isothermal elastic crystals. J. Mech. Phys. Solids, 37:293-316, 1989.

[34] D. Steigmann. Equilibrium theory for magnetic elastomers and magnetoelastic membranes. Int. J. Nonlinear Mech., 39:1193-1216, 2004.

[35] D. J. Steigmann. Tension-field theory. Proc. R. Soc. Lond. A, 429:141-173, 1990.

[36] D. J. Steigmann. A concise derivation of membrane theory from three-dimensional nonlinear elasticity. J. Elasticity, 97:97-101, 2009.

[37] D.J. Steigmann. Advances in the Mechanics of Solids, in memory of E.M. Haseganu, volume 15 of $B$, stability, vibration and control of systems Eliza Haseganu's analysis of wrinkling in pressurized membranes, pages 3-16. World Scientific, Singapore, 2006.

[38] M. Taylor and D. J. Steigmann. Entropic thermaelasticity of thin polymeric films. Acta Mech., 183:1-22, 2006.

[39] M. Taylor and D. J. Steigmann. Simulation of laminated thermoelastic membranes. J. Thermal Stresses, 32:448-476, 2009.

[40] P. Underwood. Computational Methods for Transient Analysis, chapter Dynamic Relaxation, pages 245-265. Elsevier, 1983.

[41] J. S. Yang and G. A. Maugin, editors. Mechanics of Electromagnetic Solids. Kluwer Academic, Dordrecht, The Netherlands, 2003.

[42] Q.-S. Zheng. Theory of representations for tensors: A unified invariant approach to constitutive equations. Appl. Mech. Rev., 47:545, 1994. 